\title{
Forbidden fruits
}

Citation for published version (APA):

Engels, R. C. M. E. (1998). Forbidden fruits. [Doctoral Thesis, Maastricht University]. Universiteit Maastricht. https://doi.org/10.26481/dis.19981113re

Document status and date:

Published: 01/01/1998

DOI:

10.26481/dis.19981113re

Document Version:

Publisher's PDF, also known as Version of record

\section{Please check the document version of this publication:}

- A submitted manuscript is the version of the article upon submission and before peer-review. There can be important differences between the submitted version and the official published version of record.

People interested in the research are advised to contact the author for the final version of the publication, or visit the DOI to the publisher's website.

- The final author version and the galley proof are versions of the publication after peer review.

- The final published version features the final layout of the paper including the volume, issue and page numbers.

Link to publication

\footnotetext{
General rights rights.

- You may freely distribute the URL identifying the publication in the public portal. please follow below link for the End User Agreement:

www.umlib.nl/taverne-license

Take down policy

If you believe that this document breaches copyright please contact us at:

repository@maastrichtuniversity.nl

providing details and we will investigate your claim.
}

Copyright and moral rights for the publications made accessible in the public portal are retained by the authors and/or other copyright owners and it is a condition of accessing publications that users recognise and abide by the legal requirements associated with these

- Users may download and print one copy of any publication from the public portal for the purpose of private study or research.

- You may not further distribute the material or use it for any profit-making activity or commercial gain

If the publication is distributed under the terms of Article $25 \mathrm{fa}$ of the Dutch Copyright Act, indicated by the "Taverne" license above, 
Forbidden Fruits 
Q Rutger C.M.E. Engels, Mastricht, 1998

ISBN $90-9011967-1$

The study presented in this thesis was conducted at the Nutrition Toxicology and Environment Research Institute Maastricht (NUTRIM). "The Department of Medical Sociology is associated with the Addiction Research Institute Rotterdam.

"This study was funded by a grant from the Dutch Cancer Society (grant RL 94-728). Financial support for publication of this thesis by the Dutch Cancer Society and the Netherlands Heart Foundation is gratefully acknowledged. In addition, financial support for the printing of this thesis has been kindly provided by the Department of Medical Sociology.

Production: Datawyse / Universitaire Pers Maastricht 


\title{
Forbidden Fruits \\ Social Dynamics in Smoking and Drinking \\ Behavior of Adolescents
}

\author{
Proefschrift
}

ter verkrijging van de graad van doctor

aan de Universiteit Maastricht, op gezag van de Rector Magnificus,

Prof. dr. A.C. Nieuwenhuijzen Kruseman

volgens het besluit van het College van Decanen, in het openbaar te verdedigen op

vrijdag 13 november 1998 om 14.00 uur

door

Rutger Cornelis Martinus Elizabeth Engels

geboren te Velp op 24 oktober 1968 
Promotor:

Prof. dr. M. J. Drop

Co-promotor:

Dr. R. A. Knibbe

Beoordelingscommissie:

Prof. dr. G. J. Kok (woorzitter)

Prof. dr. M. P. F. Berger

Prof. dr. ir. P. A. van den Brandt

Dr. K. Mäkelä (Finnish Foundation for Alcohol Studies, Helsinki)

Prof. dr. Th. G. W. Paulussen (Katholieke Universiteit Nijmegen) 


\title{
CONTENTS
}

\author{
1 \\ Introduction 7
}

2

Development of smoking and drinking in a cohort of secondary school children 29

3

Homogeneity of smoking behavior in peer groups:

Influence or selection? 51

4

Influences of parental and best friends' smoking and drinking on adolescent use 63

5

Intimate relationships and alcohol use:

When love comes to town 83

6

Functions of pub-going for late adolescents 97 7

Why do late adolescents drink at home?

A study on psychological well-being, social integration and drinking context 111

8

Predictability of smoking in adolescence:

Between optimism and pessimism 123

9

Antecedents of smoking cessation among adolescents:

Who is motivated to change? 139

10

Inconsistencies in adolescents' self-reports of initiation of alcohol and tobacco use 155

11

General discussion 167

References 193

Samenvatting (summary in Dutch) 211

Acknowledgements 217

Curriculum Vitae 219 
Chapter 10 deals with problems of measuring smoking and drinking behavior with self-reports. Longitudinal analyses focused on the inconsistencies in the age of onset of first use, and on the effects of errors in this concept on the prediction of future substance use.

A summary and discussion of the main findings are presented in Chapter 11. The implications of this study for future research and prevention efforts on smoking and alcohol misuse are addressed.

Chapters 2 to 10 are based on research articles which have been published in scientific journals or submitted to a journal for review. This implies that most of the chapters are written to stand alone and parts of the thesis overlap. In particular, parts of the Method sections of the chapters are quite similar as an identical data-set was used.

\section{NOTES}

${ }^{1}$ There is a focus on heterosexual relationships in this thesis. Although I do not assume that the effects of drinking on partnership are different for gay people, this is not the subject of the current research.

${ }^{2}$ Ajzen (1985, pp 22-23) acknowledged the relevance of prediction over time, but assumed that long term prediction of behavior on the aggregate level was the main topic of empirical studies. The case of smoking onset, of course, shows the opposite. 



\section{INTRODUCTION}

Why do some people take up smoking while others do not? What are explanations for the fact that some people only consume a few glasses of alcohol per week while others get drunk at every opportunity? And why do some youngsters abstain from alcohol and consequently do not benefit from possible facilitating aspects of drinking? For many youngsters, the adolescent years comprise the period in which they are confronted with potential risky behaviors, such as drinking, smoking and drug use. A large portion of adolescents experiment with these substances. Some of them continue to use them while others quit and do not go through the experimental stage. Over the past decades, social scientists have tried to understand the causes of onset of substance use, and the precursors of changes in use. Departing. from a social perspective, this thesis shed new light on some of the ideas and opinions about adolescent smoking and drinking. Young people do not start to use substances in isolation. A variety of environmental influences from peers, parents, siblings, friends, partners or the media affect young people's preferences, attitudes and behaviors. However, they are not merely innocent lambs who fall into the jaws of wolves. People learn about the norms in their surrounding networks and react to them in different ways, for instance, by adopting the behavior, influencing others or seeking other companions. So instead of studying social influences the focus of this thesis is on social interactions.

Many perceive experimentation of substance use as an evil that should be prevented at all costs. At the level of society (legal ages for buying alcohol, mass media campaigns), school (primary and secondary prevention programs) and family 
(parents who try to forbid their offspring to smoke or drink), forces are operative against risky behaviors. In addition, many research programs concentrate on the negative consequences of substance use. However, these studies do not provide much information about why, despite the obvious hazards associated with use, young people start drinking alcohol or smoking cigarettes. When adolescents are asked to give reasons for their alcohol use, they often mention the social benefits of use: it makes parties and social gatherings more fun and enjoyable, and it eases contacts with the opposite sex. Moreover, drinking can also be seen as part of a normal maturing process reflecting aspects of psychological development. The insight that social and personal dimensions of drinking are relevant, directs attention to the context of use. Since most youngsters drink in public drinking places with peers and friends, the enhancing effects of alcohol might be ascribed to pub-going. But why then do some adolescents drink exclusively at home? An explanation could be that they drink for reasons other than to enhance their social identity or establish sarisfying relationships with peers. Drinking to cope with stress and feelings of low self-esteem might be one of these reasons.

Young people's ideas, thoughts and goals concerning cigarettes and alcohol affect their "decision" to change their behavioral patterns, for instance, to try a cigarette or to continue smoking. Many studies state that cognitions, such as attitudes, self-efficacy expectations and intentions with respect to smoking and drinking are important aspects of processes leading to onset, maintenance and quitting. Nevertheless, some important questions need to be answered. For example, what is the role of smoking-specific cognitions in a constellation of other factors, such as environmental factors, in explaining smoking onset and what is the impact of cognitions besides habitual factors in predicting adolescents' motivation to quit smoking? And do cognitions predict changes in smoking not only in the short term but also in the long run?

Most of the research questions proposed in this thesis have been answered with longitudinal data from a cohort of Dutch adolescents. Inevitably, the longitudinal and quantitative character of the data raises some methodological issues. One of these is the extent to which model variables predict substance use over a period of a few years. Another issue is related to reliability and validity of adolescents self-reports on alcohol and cigarette use. In particular, questions about. the accuracy of the history of substance use can be addressed with longitudinal data.

The time limits of this project and the richness of the data of the cohort study necessitate choosing from a number of possible topics and, in most cases, being limited of a choice for one specific behavior (i.e., smoking or drinking). These choices are guided by personal interest, by the importance of further refinements of theory or prevention, or by very practical reasons (for instance, some scales in the questionnaires were only suitable for smoking or drinking). Furthermore, this introduction does not provide an extensive and complete overview of theories or empirical research on changes in substance use of young people. Only those theories 
that are applied in the following chapters are discussed (see for a thorough and recent review of theories, Petraitis, Flay \& Miller, 1995). In addition, in analyses carried out exclusively for smoking or drinking, the empirical research applicable to this behavior is discussed.

This chapter will start with a discussion of the research on social influences and adolescent smoking and drinking. In particular, the role of selection effects and social interaction in this respect will be addressed. Second, the social functions of drinking for late adolescents, and the research done on this issue, will be addressed. Third, smoking-specific cognitions and their relationships to smoking onset and smoking cessation will be discussed. In addition, some topics which to a certain extent come up in all chapters are addressed. The first issue is the role of habit formation and addiction in the development of adolescent smoking and drinking. The second issue is the additional value of a longitudinal design in studying the turmoil of adolescence. A third issue is related to differences and similarities in processes leading to smoking and drinking. This chapter will end with a short description of the other chapters in this thesis.

\section{INFLUENCE, SELECTION AND SOCIAL INTERACTION}

\section{Social Influence Processes}

Several theoretical perspectives have been postulated to explain the social context in which young people initiate and maintain drinking, cigarette smoking and drug use. The social learning theory (Bandura, 1977, 1986) states that social behaviors are learned by observation. It postulates that social agents affect individual behavior by setting an example and by providing reinforcement. Whether deviant or conforming behavior is acquired depends on past and present rewards or punishments related to the specific behavior and the rewards and punishments attributed to alternative behaviors. The consequences of a behavior can be social (rewards, group membership or becoming an outcast) or non-social (physiological effects of substance use)(see Bandura, 1977).

The social learning theory assumes a model which orders and specifies the relations between variables (see also Akers, 1979; Massey \& Krohn, 1986). First, people are supposed to act in social groups which entails exposure to rules, perception of models and social reinforcement. The interaction with using and nonusing models, and the anticipated mix between positive and negative consequences, induce initial use or continued abstinence. When people have started to use a particular substance, modeling becomes less important, whereas the social and physiological reinforcers and punishments become more relevant. For example, when a youngster notices that his or her friends enjoy the pleasures of drinking together, and $s /$ he experiences some relaxation effects from alcohol, $s /$ he is more likely to continue this behavior (see also Abrams \& Niaura, 1987; Kandel, 1980). In 
their differential reinforcement theory, Akers and co-workers (1979) applied and extrapolated the ideas of Bandura into the field of adolescent substance use. In particular, the value of their contribution consisted of a differentiation in sets of mechanisms of influences. Imitation (number of important others), differential association (peer and parental attitudes, norms and behaviors), differential reinforcement (social rewards and punishments) and non-social attitudes were the core factors in their approach.

Although Bandura and Akers provided an initial look at the functioning of social influence processes, little is still known about the nature of these process in empirical research (Graham, Marks \& Hansen, 1991). These authors tested a framework which identified two types of influence. First, active social pressure consisting of offers and explicit pressures to use a substance. This type of pressure impinges directly on an adolescent and demands for an immediate response. Second, passive social influences refer to modeling of behavior. Young people receive normative information about a behavior by observation and by conversation with peers. Another form of passive influence is the misperception of peer use. This latter mechanism refer to the accuracy of recall of peer use. Studies on smoking reveal that youth at different stages of onset all overestimate smoking prevalence but the tendency to overestimate peer and friends' use is stronger among smokers (e.g., Bauman \& Ennett, 1996; Mäkelä, 1997; Marks \& Miller, 1987; Sherman, Presson, Chassin, Corty \& Olshavsky, 1983). Graham et al. (1991) examined their model longitudinally in a sample of school children. Support was found for the distinct social influence processes on changes in smoking and drinking.

Turner (1991) advocates a somewhat different classification of social influences, separating descriptive from injunctive norms. The former norms refer to the actual or perceived behavior of others, and are similar to the modeling construct. Injunctive norms, on the other hand, specify what people approve and disapprove (see also Cialdini et al., 1990). Fishbein and Ajzen (1975) also emphasized the relevance of social norms. In their theory of reasoned action, people's perception of the opinion of important others and their motivation to comply with these opinions, are assumed to affect behavioral intentions. Extrapolating this model by including modeling and overt pressures, De Vries, Backbier, Kok and Dijkstra (1996) found evidence for the effects of these three variables on changes in smoking behavior amongst adolescents. In addition, Wilks, Callan and Austin (1988) and Biddle, Bank and Marlin (1980) showed the independent effects of norms and perceived behavior on adolescent drinking.

Although it is essential to scrutinize the ways people exert influence on each other, it should be noted that these processes are not mutually exclusive. For instance, it is most likely that those who offer cigarettes smoke themselves. Certainly the attempt to persuade someone to smoke will be less powerful if made by a non-smoking peer. A similar argument can be made for the association between modeling and norms. It is not very surprising that these factors are 
correlated. In the study by De Vries et al. (1996), social norms and perceived behavior correlated .30. Since they used a composite measure, adding modeling effects of several 'significant' others, the associarions of norms and perceived behavior for the individual persons (e.g., peers, parents, siblings) might even be higher. In our own study, subjective norms of parents $(r=.39)$, friends $(r=.34)$ and partner $(r=.33)$ are significantly $(p<.001)$ related to the smoking status of the 'individual' persons (unpublished data). In sum, these associations suggest that the effects of social norms, explicit pressures or offers on smoking behavior are less likely in the absence of modeling influences.

\section{Socializing Agents}

A variety of sources of influence are mentioned in the literature. Most prominent are the opinions, values and behaviors of persons in the immediate environment of youngsters. Parents and other family members, such as aunts, uncles or grandparents are recognized as potential role models (Petraitis et al., 1995). Parental attachment and affective ties, especially in early adolescence, are expected to enhance the possibility that childrens' ideas and behaviors conform to those of their parents. Thus, when youngsters grow up in a smoking family environment, it is not surprising that they consider this behavior to be normal and almost normative, and start to smoke themselves (Krosnick \& Judd, 1982).

In addition, siblings, and especially older siblings, have been characterized as a category of peers who can act as a source of imitation (Flay et al., 1994; Goddard, 1990; Needle et al., 1986). Schoolmates, best friends and the cluster of close friends are important persons in adolescent lives. According to Oetting and Beavais (1986), the troubled young person, living through the turmoil of adolescence, is probably vulnerable in his or her need to join or remain in a group. Peers shape attitudes about alcohol and smoking, provide the social context for the use of these substances and help to form the rationales for the use or non-use of drugs.

Another source of influence is formed by the tobacco and alcohol industries. Although they are not allowed to concentrate their actions on youth, advertisements and sales promotions might still have an effect on initiation and maintenance of alcohol and tobacco use. On the other hand, government policies aimed at restricting drinking and smoking among youngsters. Primary prevention programs at school and (national) media campaigns are developed to inform them about the social and health consequences, and to teach them to resist social pressures and tempting situations (e.g., Bruvold, 1993; Flay, 1985; Glynn, 1989). Some researchers point to teachers as a key figure in the adoptation of risky behaviors (Molimard, 1994). In particular, a few decades ago, when the smoking status of teachers was salient to pupils (e.g., it was quite common that teachers smoked in classrooms), they might have had some impact on the taking up of smoking of their students. Furthermore, in late adolescence, when the majority of youngsters spend 
considerable time at the workplace, colleagues and supervisors are potentially relevant role models. Finally, intimate relationships are of growing significance in adolescence. Several studies have stated the influence of steady and fluctuating relationships on teenagers' well-being, communicative skills and behaviors (e.g., Samet \& Kelly, 1987; Sanderson \& Cantor, 1995).

\section{Changes in Relative Influences}

There is an ongoing debate about the impact of parents and peers on changes in smoking, drinking and drug use (e.g., Aseltine, 1995; Conrad, Flay \& Hill, 1992; Engels, Kribbe, Drop \& De Haan, 1996; Engels, Knibbe \& Drop, 1997a; Flay et al., 1994; Hu, Flay, Hedeker, Siddiqui \& Day, 1996; Wilks, Callan \& Austin, 1989). Most researchers do not discuss the question of whether there is a single influential party - the extent to which one influence or another is relatively stronger is a topic in many projects. Therefore, knowledge on whether parents or peers have more effect on adolescent substance use is needed, not only for theoretical purposes but also for social policies and prevention efforts (Biddle, Bank \& Marlin, 1980; Flay et al., 1994).

Some researchers have stressed that attachment to parents accounts for initiation of substance use mainly in early adolescence (see Baumrind, 1987; Knibbe \& Van de Goor, 1988). When youngsters are in their teenage years they usually start to question the previously accepted attitudes and behavioral guidelines of parents (Baumrind, 1987; Pape, 1997). In addition, Kandel and Andrews (1987) argue that changes in influences are due to the weakening bonds with parents and increasing bonds with peers in the course of adolescence. The leveling of social bonds and control by parents could, for some adolescents, lead to the association with peers who use substances and encourage substance use among other peers (Hirschi, 1969). During the course of adolescence, young people invest considerable time and energy in friendships (Keefe, 1992). As the adolescent strives to achieve independence from parents, close peers become an important source for defining oneself and assuring self-worth. Belonging to a peer group with its rituals and behavioral requisites is meaningful for identity formation and personal well-being of youngsters (Gordon, 1986). Considering the growing role of peers and close friends in adolescent lives, substance-related norms and behaviors of friends are expected to become increasingly important.

Nevertheless, no conclusive evidence has been obtained about the relative impact of parents versus peers. In a review of longitudinal studies on smoking onset, Conrad, Flay and Hill (1992) stated that, in general, peers and friends had more impact on the initiation of smoking than parents. However, there are only a few studies that have followed a cohort of youngsters during their formative years. Cross-sectional and prospective studies with a short follow-up (one or two years) 
can not offer substantial information about a relative decrease or increase in peer and parental influences.

\section{Birds of a Feather}

Homogeneity of smoking and drinking in peer groups is mostly ascribed to peer influences. However, similarities in behaviors could also be due to interpersonal selection. Selective association refers to the tendency of youngsters to seek out and affiliate with others who have a similar life-style. Two different mechanisms of selection can be distinguished. First, adolescents acquire new friends with similar opinions, attitudes and behaviors. Second, adolescents can, on the other hand, turn down contacts with potential new friends or break off friendships because of experienced differences (i.e., deselection). As Bauman and Ennett (1996) stated, the selection model postulates that substance use causes friendships while the influence model postulates that friendships cause substance use: the causality might thus be in opposite directions. Besides this theoretical issue, the notion of selection offers another view of adolescents acting in social relationships. Youth are not left at the mercy of environmental influences but also play an active role in friendships. It is rather peculiar that although Kandel (1978) and Cohen (1977) had already pointed to the confounding effects of selection and influence when testing similarities in behavioral patterns twenty years ago, selection processes did not acquire more attention. Most research reports, (see for example the review of longitudinal smoking studies by Conrad et al., 1992), did not include a control or test of possible interference of selection effects.

Acknowledging selection effects has theoretical consequences, because peer influence is widely believed to be one of the most important sources of involvement in alcohol, smoking and drug use in theoretical and empirical studies (e.g., Petraitis et al., 1995; Werner, 1991). The belief that peer influence is a strong determinant of the initiation of risky behaviors has been set a trend among health education and prevention workers. Most primary prevention programs have included elements in which youngsters are trained to resist peer pressures. However, if similarities in peer groups are to a large extent attributable to associative pairing, current educational programs focusing on peer factors might be of little value in preventing smoking or drinking.

Assessment of selection effects requires specific designs and measures. Crosssectional studies can not provide insight into selection effects. Information of respondents and their social network is required at different time points; data on changes and stability in behaviors and networks are necessary (Ennett \& Bauman, 1994). Studies with a better methodology have shown that selection effects are underestimated and that peer factors are overestimated in affecting homogeneity in drinking behavior (Fisher \& Bauman, 1988; Urberg, Degirmenciogly \& Pilgram, 1997), smoking (Ennett \& Bauman, 1994; Fisher \& Bauman, 1988), drug use 
(Kandel, 1978), political preferences (Cohen, 1977) and sexual behavior (Billy \& Udry, 1985). In most of these studies, researchers examined influence and selection processes by following stable and unstable dyadic friendships. Because once stable friendships are established, selection by definition can not occur, initiation of, for instance, smoking by a respondent with a smoking friend as well as quitting by a respondent with a non-smoking friend, can only mean that peer influence is operating. Although selective association appears to be a strong factor, only limited support was found for deselection. Differences in substance use seem to be no reason for breaking off a friendship or stepping out of a peer group (Billy \& Udry, 1985; Ennett \& Bauman, 1994; Urberg et al., 1997).

Although efforts have been undertaken to come to a more accurate test of the interpretation of homogeneity in smoking and drinking, there are several shortcomings. First, most of the network studies have focused on school children. This means that peer contacts outside the school are ignored. Second, despite the longitudinal character of these studies, only few of them had a long period of time between the first and second wave. In order to have a large variation in changes in peer group constellation or close friends as well as changes in smoking or drinking, a few months between the waves is most likely not enough to come to an appropriate test of hypotheses.

As mentioned before, a feature of controlling selection processes is that analyses might show that influence processes are less important in predicting changes in substance use than is assumed by many researchers. This leaves more room for other factors which are currently undervalued. For instance, the discussion about parent and peer influences might get some fresh blood by the notion of selection. It is possible that parental influences are of comparable or more value in affecting initiation and maintenance of smoking and drinking when selection effects are incorporated in an analytic model.

The relevance of social interaction can be underscored if we focus on the role of family members in friendships. Parents do not only affect drinking and smoking habits of their offspring but also seem to exert their impact on peer preferences (e.g., Hansen et al., 1987). Anti-smoking or drinking sentiments could lead to parents prohibiting their children associating with "bad company" or preventing them going out to pubs and discos. More implicitly, internalization of the life style of parents and socialization processes result in normative beliefs about with whom to affiliate and with whom not to.

Thus, the existing research is virtually uniform in its orientation towards potential socialization forces which act upon youth, while minimizing the role of individuals in affecting their environment (Aseltine, 1995). Nonetheless, the function of adolescents themselves in their relationships with friends and parents, as well as the environmental and cultural features in which friendship choices and peer behaviors develop might be crucial in understanding the relative value of unidirectional influences. 


\section{SOCIAL FUNCTIONS OF DRINKING}

\section{Beneficial Features of Drinking}

Adolescent drinking has several negative consequences, such as drunk driving or cycling (e.g., Brouwer \& Kingma, 1995; Hingson, Heeren, Howland \& Winter, 1991; Passies, 1991), aggression and violence in and around public drinking places (Milgram, 1993; Van de Goor, 1990) and sexual harassment (Leigh, Schafer \& Temple, 1995). In many cases, these side effects of drinking cause physical, psychological and social problems for youngsters and their immediate environment. Nevertheless, these findings do not elucidate why the majority of older adolescents go out regularly and drink allcohol. Why do some people stay sober whereas the majority of teens start consuming alcohol? 'To gain insight into the functions of drinking one should focus on the facilitating effects of alcohol. Four dimensions within these so called beneficial effects of drinking can be distinguished, namely, (a) transitions to young adulthood, (b) social integration or sociability, (c) development of intimate relationships and (d) psychological well-being.

Alcobol as a Transition Marker. An almost provocative statement of Jessor (1987, p. 335 ) is that some behaviors such as smoking, sexual intercourse and drinking are "not necessarily irrational, perverse or pathological; for adolescents, such behaviors can fulfill important goals and can be an essential aspect of psychosocial development." Furthermore, the initiation of drinking can be seen as a rule rather than an exception and most of the time normal rather than deviant (Bagnall \& Plant, 1987; Van de Goor, 1990). The adolescent years are signified by an increasing attention for adult-like behaviors and opinions (Maddox, 1962). By looking at how adults act, and how they are portrayed in the media, teens learn about the norms and values of adults. Because drinking is a rather normative behavior among adults, it is not strange that youngsters adopt this habit. Financial independence through a job (on the side), an interest in a future position in society in terms of employment, dating and mating, and leaving the parental home are other aspects, besides drinking, of an orientation towards the adult world. Other changes in adolescent lives are independency, autonomy and a clear self-definition (Erickson, 1969). There is some empirical evidence that drinking goes hand in hand with other changes in orientations. Jessor and Jessor (1975) stressed that abstinence might be associated with a delayed separation process. In comparison with their peers, abstainers are more affected by traditional values. (i.e., school, religion) and by their parents. In their longitudinal study, the authors found that young drinkers were less likely to be involved with and bonded to their parents, and experienced less influence from traditional institutions than abstainers. Thus, accepting parental supervision without questioning by late adolescent abstainers might be regarded as a sign of late maturation. 
Peer Contacts and Drinking. As mentioned before, the transition from adolescence to adulthood is characterized by changes in social relationships. Teens spend more time with peers and less time with family members (Schulenberg et al., 1996). In addition, closeness and intimacy become important features within adolescent friendships - personal problems and experiences are more often discussed with friends who are in a similar situation. Being a member of a social group is essential for the emotional and psychological development of teens (e.g., Keefe, 1992). When youngsters are asked what motives they have for drinking, they often mention the social aspects of drinking. Drinking seems to make parties more fun, it makes one more relaxed, makes it easier to approach others and to share feelings and experiences. Research on alcohol expectancies has shown that the expected reinforcing social elements of drinking are related to, and predictive of, frequency and quantity of adolescent alcohol use (e.g, Brown, Creamer \& Stetson, 1987; Christiansen, Goldman \& Inn, 1982; Leigh \& Aas, 1995). Apparently in the eyes of the beholder (i.e., youngsters themselves), drinking is related to sociability and connected to social events. Indeed some support is found for the social function of drinking. An Australian study (Winefield, Winefield, Tiggeman \& Goldney, 1989) reported that moderate drinking appeared to be a normative behavior for the welladjusted and socially integrated young adult. In a study of Swedish conscripts, Leifman, Kühlhorn, Allebeck, Andréasson and Romesjö (1995) reported that abstainers were less sociable than drinkers (i.e., light, moderate and heavy drinkers). Silbereisen and Noack (1988), Reid (1978) and Pape (1997) reported that abstainers spent less time with their friends and were less likely to have a close friend. Pape also indicated that abstainers had poorer social skills compared to drinkers. A Dutch study in the 16-24 age-category showed that sociability was positively related to the frequency of drinking (Oostween, Knibbe $\&$ De Vries, 1996).

Intimate Relationships and Drinking. Love and romance are major concerns for young people. In this period of life, they become interested in the opposite sex and have their first dates and sexual encounters. ${ }^{1}$ Although teen relationships are mostly short and sequential, they are important for their self-identity, emotional development and well-being (Paul \& White, 1990; Sanderson \& Cantor, 1995; Weinstein \& Rosen, 1991). In addition, the start of intimate relationships can be seen, just like the consumption of alcohol, as one of the behaviors marking the transition from childhood to young adulthood. The few (prospective) studies which examined the effects of drinking on partnership indicated a positive association -abstainers, or late starters, were less likely to have a steady partner (Pape $\&$ Hammer, 1996). An explanation for this association is obviously related to the context of drinking. Silbereisen, Noack and von Eye's (1992) study among Berlin teenagers showed that pubs and discos were settings which provided the possibility of establishing a relationship or having a romantic affair. It should be stressed, however, that drinking does not lead to a higher likelihood of having a steady partner in all circumstances. According to Newcomb and Bentler (1988), it is 
possible that heavy or problem drinking could be a sign of a life-style which does not appeal to others, and is associated with decreasing chances of involvement in a relationship.

Psychological Well-being and Drinking. Drinking with peers facilitates the sharing of activities, experiences and emotions with them. The exchange of common experiences and the knowledge that others are in a simillar position have a positive impact on youngsters' well-being (Dolcini \& Adler, 1994; Hartup, 1983). For instance, engagement in social activities with friends seems to be associated with healthier emotional functioning (Windle \& Windle, 1996). This point of view is related to the ideas of Pape and Hammer (1996) on alcohol abstinence in late adolescence and young adulthood. They emphasize that alcohol use is the norm for people in this period of life. Deviating from the mainstream seems to be associated with negative aspects, such as a lack of social integration as well as low self-esteem and feelings of depression. In their opinion, there could be an u-shaped association between well-being and drinking, with lower scores on psychological well-being for both abstainers and heavy drinkers.

\section{Drinking Context}

In Western societies, young people's alcohol consumption is concentrated in peer settings, such as public drinking places, parties, pubs and youth centers (e.g., Harford \& Grant, 1987; Iversen, 1996; Knibbe, Oostveen \& Van de Goor, 1991). Attention for the purposes of pub-going may shed some new light on the assumed beneficial effects of drinking. Perhaps these effects should, to a large extent, in fact be ascribed to pub-going. However, the strong association between pub-going and drinking makes it hard to examine whether, or which, beneficial functions of drinking could be attributed to the situation in which people drink. On the other hand, the combination of being at the right place and the consumption of a few glasses making an individual more relaxed and spontaneous, might explain the social effects of drinking.

The social function of (drinking in) public settings leads to the question of why some youngsters drink exclusively at home. Because it is not likely that drinking with parents or alone has an impact on peer relationships, they might have other reasons for drinking. The work of Pierce, Sarason and Sarason (1992) on stress and drinking might be helpful. The authors hypothesized that whether stress leads to deviant behavior depends on the amount of social support people receive in a given situation. If people have problems in life and do not get support from important orhers, such as parents, friends or a partner, they are prone to look for alternatives to deal with stress. Following this line of reasoning, one might speculate why some youngsters drink in non-peer settings, such as the parental home (with parents or alone). Dealing with stress and low self-esteem might be one of the reasons involved. 


\section{SMOKING-SPECIFIC COGNITIONS}

\section{Attitudes, Selfefficacy and Intentions}

The attention to social psychological models which focus on the cognitive aspects within the development of substance use patterns has been growing in the past twenty years (see Flay \& Petraitis, 1995; Sutton, 1987). The basic proposition is that most of people's activities are designed in advance - actions are controlled by intentions, plans and goals. With respect to smoking, it is assumed that prior to smoking onset in youngsters there is a process of contemplation in which they evaluate the conditions, eventual social responses, arguments and personal goals. Much of the work on this topic has been inspired by the theory of reasoned action that deals with relations between beliefs, attitudes, intentions and behavior (Fishbein and Ajzen, 1975). According to this theory, intentions to perform any behavior are strongly affected by the persons' attitude towards the behavior. This attitudinal component is a summative measure of expectancy-value beliefs on the pros and cons of a behavior. Applied to adolescent cigarette use, it is expected that youngsters with strong pro-smoking beliefs and weak anti-smoking beliefs have the highest intentions to initiate or maintain smoking.

Ajzen $(1985,1991)$ responded to one of the main criticisms about this model, namely the limitation to behaviors which are under volitional control, by adding the concept of self-efficacy. People's expectations of efficacy (i.e., having the skills, abilities and opportunities) to change behavioral patterns are assumed to be, the third determinant of behavior intentions after social norms and attitudes. Selfefficacy takes two forms (Petraitis et al., 1995). Use self-efficacy represents people's beliefs in their abilities to obtain and perform a behavior (i.e., smoking). Second, refusal self-efficacy refers to people's beliefs in their abilities to resist social pressure to begin or maintain using a substance. The serious health consequences of smoking, its self-selected voluntary nature and the prevalence of early onset among youngsters underscore the potential utility of the concept of self-efficacy in explaining the decisions of adolescents to take up smoking (Lawrance $\&$ Rubinson, 1986).

\section{Smoking Onset}

The contents of beliefs vary from smoking for pleasure and relaxation, impressing others, reducing stress, changing image, or feelings of autonomy (e.g., De Vries, 1989; Grube, Rokeach \& Getzlaf, 1990; McGee \& Stanton, 1993). Reasons for not smoking are related to health issues, taste, opinions of parents and peers, and financial costs. The general picture is that beliefs or attitudes are one of the most consistent predictors of smoking (see Conrad et al., 1992). Thorough studies with large samples following high school students in different age categories reported that 
attitudes predicted smoking one year later (Ary \& Biglan, 1987), 4 and 16 months later (Collins et al., 1987), 2 years later (Chassin et al., 1981) and 5 and 8 years later (Pederson \& Biglan, 1987). Some researchers have reported mixed results when they looked at different beliefs. McNeill et al. (1988), for instance, showed that knowledge of short-term harmful effects did predict smoking onset whereas knowledge of long-term harmful consequences did not. In their set of items, the strongest effects were found for intentions to smoke, smoking for pharmacological reasons and approval by parents. McGee and Stanton (1993) reported that the immediate negative consequences were a reason for non-smoking, while reasons for smoking were not associated with later smoking.

Flay and co-workers (1994) tested a structural model which integrated peer and parental use with the concepts of the theory of planned behavior (Ajzen, 1991). In prospective analyses, they found direct effects of intentions to smoke on actual smoking behavior one year later, and effects of refusal self-efficacy, parental norms, and negative outcome expectations on intentions (and not directly on smoking onset). De Vries et al. (1995) reported the relevance of attitudes, self-efficacy and intentions on smoking onset over a period of 18 months. In addition, Lawrance and Rubinson (1986) reported independent effects on smoking onset over periods of 3 and 5 months of three self-efficacy (scales involving social opportunities to smoke, emotional stress and peer influences).

\section{Smoking Cessation}

Although numerous studies have dealt with the effects of attitudes and self-efficacy on smoking onset, the impact of cognitions on quit attempts, motivation to quit and actual smoking cessation has largely been unaddressed in adolescent samples (Chassin, Presson \& Sherman, 1984; Stone \& Kristeler, 1992).

An explorative study by Stone and Kristeler (1992) revealed that the reasons adolescent smokers have for wanting to quit comprised healsh reasons, difficulties with refraining from the addiction, and social costs in terms of problems with friends. In contrast, according to Thambypillai (1987), awareness of the health risks was not profound enough to cause current smokers to quit (see also Tuakli et al., 1990). Nonetheless, this variation in findings might result from differences in the awareness of health risks and the presence of health reasons to quit. There is some evidence for the long term impact of attitudes on smoking cessation. In a seven year prospective study, Rose and colleagues (1996) showed that certain motives for smoking in adolescence were related to whether smoking cessation was successful in young adulthood. The enjoyable and stimulating features of smoking in particular, affected cessation. In addition, Chassin et al. $(1984,1991)$ found that both psychological and health reasons were predictive of smoking persistence. Hansen, Collins, Johnson and Graham (1985) showed that belief in, and desire for, positive 
short-term consequences (relaxation, social benefits, no interest in long term health) affected quitting one year later in high school students.

There is a lack of knowledge of the role of self-efficacy in the quitting experiences of young smokers. However, self-efficacy expectations have been widely adopted in the field of adult smoking, in theoretical issues as well as in secondary prevention programs (e.g., DiClemente et al., 1991; Prochaska, DiClemente \& Norcross, 1992; Strecher, DeVellis, Becker \& Rosenstock, 1986). In general, studies have underscored the association between levels of perceived behavioral control and quitting attempts in a sample of unaided quitters (Carey \& Carey, 1993; Lichtenstein \& Cohen, 1990), long term success among unaided quitters (Eiser, Morgan, Gammage, Brook \& Kirby, 1985) and cessation in a group program (Mudde, 1994) or with tailored interventions (Dijkstra, De Vries \& Rooyackers, 1997).

\section{Criticism}

The interpretation of relationships between smoking-related cognitions and smoking behavior has been critized, both on methodological and conceptual grounds (Eagly \& Chaiken, 1993; Fazio, 1989; Leigh \& Aas, 1995; Sutton, 1987). Some of these criticisms will be discussed. First, the question of causality remains largely unanswered. To what extent are the attitude-behavior relationships due to the preceding effects of, for instance, experimental smoking on the formation of attitudes later on (e.g., Bentler \& Speckart, 1981; Eagly \& Chaiken, 1993; Kelman, 1974). Stacy, Widaman and Marlatt (1990) stated that findings on attitude-behavior relationships are mostly based on cross-sectional studies. These studies are more likely to be spurious because of method biases or self-perception apparent at a single time of measurement. From the perspective of cognitive dissonance theory (Festinger, 1957), it is plausible that people who start smoking but still have a negative attitude towards smoking, are more likely to change their attitudes in order to reduce dissatisfaction and negative feelings about themselves. Prospective studies are intrinsically more powerful for drawing conclusions about the causal order of variables (Jarvis, Goddard \& McNeill, 1990). Longitudinal studies on this topic using sophisticated analytic methods, such as structural modeling, suggested that attitudes were influenced by experiences with the behavior (Stacy, Bentler \& Flay, 1994). In a survey among $11^{\text {th }}$ grade high school students, the authors showed that smoking at Time 1 predicted attitude scores at Time 2 as well as smoking at Time 2. However, attitudes at Time 1 were not predictive of future smoking at Time 2 . Leigh and Aas (1995) suggested that especially in the first phase of smoking, when people do not have earlier experiences, attitudes affect the uptake of this behavior. This coincides with the ideas of Bem (1978) that in the case of stable behaviors (like maintenance of smoking), beliefs about the pros and cons of smoking are mostly determined by behavior. 
Second, one could question whether smoking onset is a very deliberate decision process. According to Fazio (1986), an individual's construction or definition of the events affects his or her behavior, especially by weak attitudebehavior links. This process is far less deliberate and far more spontaneous than expected (p. 237). Only when people are well aware of their attitudes, can behavioral choices be guided by a deliberate reasoning process. The degree to which changes in smoking are under volitional control has also to do with the position of current and past behavior (Sutton, 1987). Since cigarette smoking is highly addictive, even among adolescent smokers (Stanton et al., 1992), it is likely that smoking involves a strong habitual component and is only partly controlled by cognitions. Bentler and Speckart (1979) added past behavior to the theory of planned behavior as a predictor of intentions and future behaviors. In particular, habitual behaviors are so routinized through repetition that a person has ceased to make any conscious decision to act (Triandis, 1980). In other words, daily smokers do not go through a process of contemplation with each cigarette they light.

Third, Fishbein and Ajzen (1975) stressed that a condition for appropriate measurement of the relationship between cognitive determinants and behavior was temporal contiguity. "The assessment of precursors should be as close as possible to the performance of the behavior. ${ }^{2}$ However, the utility of theoretical madels is very limited if violations of this methodological assumption severely diminish the correlations ( $R$ andall \& Wolff, 1994). Furthermore, one can question the practical value of this model for intervention and prevention purposes if even a short temporal delay leads to detrimental effects of cognitions on prediction of behavior. This problem especially exists in the case of smoking, where primary prevention is focused on youngsters' attitudes and self-efficacy expectations, months or even years before they start to smoke.

\section{HABITS}

\section{What about the Addictive Aspects?}

An examination of possible predictors of changes in substance use leads to the inevitable question about the role of earlier use in the constellation of factors. From research on adolescents it is known that with longer smoking experience, it becomes increasingly more difficult to refrain from smoking (Pierce \& Gilpin, 1996). For many youngsters, regular smoking is an addiction that they will not easily escape from. Physiological and psychological dependence on smoking plays a strong role in affecting future use - stronger than the young smokers themselves assume. Using the Fagerstrom Tolerance Questionnaire (Fagerstrom, 1991) or the DSM-III-R criteria in order to assess nicotine dependence, Prokhorov, Pallonen, Fava, Ding and Niaura (1996), Stanton (1995) and Breslau, Kilbey and Andreski (1994) have shown that a large proportion of (late) adolescents are dependent and 
that this should be taken into consideration in treatment or other cessation programs. McNeil (1991) indicated that nicotine played an active role in reinforcing smoking in children. It is important to stress that this does not imply that children are hooked after the first cigarette (Sutton, 1992). The British OPCS study demonstrated that about $50 \%$ of the pupils who had tried smoking once did not continue smoking two years later (Goddard, 1990). Nonetheless, there is strong evidence that those who experience the social and physiological benefits for some time are more likely to become regular smokers.

Research on transitions in frequency and quantity of alcohol use does not give a clear picture on whether certain adolescent drinking patterns lead to habit formation, heavy drinking or problem drinking (e.g., Fillmore, 1988). Some findings, however, persist across studies. Abstinence in mid-adolescence is relatively stable over the years. Moreover, once youngsters start drinking they are not inclined to become abstainers at later ages (Grant, Harford \& Grigson, 1987) quitting is not an issue for most adolescents. In addition, Temple and Fillmore (1985-86), Grant et al. (1987) and Pape and Hammer (1996) did find some support for the consistency of the relative drinking position over time. According to Pulkkinen and Pitkanen (1994), social drinking in early and mid-adolescence was not correlated with problem drinking and negative alcohol related consequences whereas alcohol dependence was. This coincides with studies of Beck et al. (1994) who argue that the reasons people have for drinking affect whether they become heavy or problem drinkers and not the drinking levels per se. Youth who drink to reduce feelings of distress or poor psychosocial maladjustment might learn to perceive drinking as a coping mechanism and might be more prone to higher drinking levels and problem drinking in late adolescence and young adulthood.

\section{Earlier Use in Analytic Models}

When earlier use is incorporated in explanatory models of smoking and drinking, it explains much of the variance in use at a later moment in time (Collins et al., 1987; Fergusson, Lynskey \& Horwood, 1994; Graham et al., 1991; Pederson \& Lefcoe, 1987; Urberg, Cheng \& Shyu, 1991). In their paper on changes in smoking, Pederson and Lefcoe concluded that such a model was parsimonious and not particularly useful for prediction. Nonetheless, one could also argue that once people start to smoke, habitual factors are stronger than other factors. An argument against neglecting earlier use is that the utility of other variables, with shared variance with earlier use, might be overestimated. For example, ignoring past behavior while testing the effects of intentions might eventually lead to incorrect conclusions due to the association between the two variables. It is also important to notice that the problem of earlier use is not a problem if one chooses to predict the transition from non-smoker to smoker (see Chapter 8; Chassin et al., 1984). However, the opposite is true for smoking cessation or reduction of drinking levels. 
In particular because several studies have indicated that differences in consumption, or level of addiction, affect people's efforts to change their habits (for instance see Dutch studies on smoking cessation by Dijkstra et al., 1996 and Willemsen, 1997, and on drinking by Hajema, 1998).

From a prevention perspective, it is highly relevant to examine the stability and continuity of smoking and drinking over time. Research on the transition from experimental smoking to regular smoking, or the transition from regular drinking to heavy or problem drinking, is instrumental for the development of primary prevention programs. If, for instance, on the individual level, drinking in middle adolescence is not predictive of drinking levels in late adolescence or young adulthood (e.g., Plant, Peck \& Samuel, 1985; Temple \& Fillmore, 1985-86), it is hard to decide on what basis, and for which target groups, interventions should be developed.

\section{THE RELEVANCE OF LONGITUDINAL RESEARCH}

\section{Phases and Stages}

Leventhal and Cleary (1980, see also Hirschman, Leventhal \& Glynn, 1984) suggested that becoming a smoker involved a set of stages which an individual should go through. First, there is a preparatory stage during which attitudes towards a particular behavior are formed and modified. Second, there is an experimental stage in which people try a substance. Subsequent social and personal rewards and punishments affect whether people proceed or quit. As an individual continues to use alcohol or cigarettes, $s /$ he enters the stage of regular use. Though there might be several paths to progress through these stages, most adolescents fit in this developmental-stage approach, according to the authors. In order to gain insight in the developmental continuity of smoking and drinking in adolescence, prospective data is needed in which young people's rransitions in behaviors are tracked. So far, stage-approaches are mostly oriented towards the onset of adolescent substance use.

In the field of adult smoking cessation, several perspectives have been proposed consisting of motivational elements, addiction and habitual factors, and actual quitting behavior (e.g., DiClemente et al., 1991; Farkas et al., 1996; Prochaska, DiClemente \& Norcross, 1992; Werch \& Anzalone, 1995). One of the most important outcomes from the research on stages of smoking cessation concerns the heterogeneity of the group of smokers with respect to their motivation to quit. Prochaska and colleagues postulated that people could move from precontemplation, contemplation and preparation to action, and then to maintenance or relapse. In terms of smoking cessation, this implies, for instance, that precontemplators are not considering quiting while preparators are planning to quit in the near future. The general assumption is that smokers in these stages 
demonstrate a developmental sequence of movement towards smoking cessation. Furthermore, it is assumed that moving from one stage to another is accompanied by differences in events and activities that facilitate the successful change of a problem behavior (DiClemente et al., 1991). It is important for prevention practice to identify the factors which differentiate between stages of cessation. An advantage is that smoking interventions can be tailored to the individual's position in a specific phase of cessation. Nevertheless, the empirical research on stages or phases in smoking cessation has been carried out in adult samples - most adolescent studies have simplified quitting to a dichotomous outcome variable (see for an exception, Pallonen, Murray, Schmid, Pirie \& Luepker, 1990).

\section{Causality}

According to Aseltine (1995), it is not only important what kind of influences people perceive but also how they themselves act on these influences. Since assessment of selection and influence processes requires data on changes in behavior, group memberships and friendships, insight in social influence processes can primarily be gained with a longitudinal design (Bauman \& Ennett, 1996). With a cross-sectional design, no definite conclusions can be drawn about an association between smoking by others on the one hand, and respondents' intentions or behavior on the other hand. In addition, no information about the causal link between substance-related cognitions and substance use can be obtained by a transversal study (e.g., Chassin et al., 1984; Flay et al., 1994). However, prospective studies do not necessarily solve problems involving the reciprocal associations between cognitions and behavior.

One of the methods to examine interrelations over time has been proposed by Bentler and Speckart (1979) by using structural modelling as an analytic strategy and with the inclusion of cross-lagged paths. The strategy is less complicated for the prediction of smoking onset. For non-users, with no or very limited experience with the consequences of use, the effect of, for instance, attitudes on smoking onset can be ascribed to the impact of attitudes (Leigh \& Aas, 1995). The strategy is more complicated for the prediction of continuation of use. An analytic strategy can comprise of the prediction of use at time 2 using a hierarchical multivariate analysis in which smoking at time 1 is included in step 1 and attitudes in step 2.

\section{Predictability}

In their review of theories on adolescent substance use, Petraitis et al. (1995) are quite optimistic about the knowledge gained on the set of variables related to the onset of substance use. However, most of the 'evidence' is based on cross-sectional studies or prospective studies with a relatively short follow-up (i.e., 1-year or less). At the same rime, some longitudinal studies have indicated that only a few percent 
of the variance in onset of smoking can be explained by the model variables employed (Collins et al., 1987; Graham et al., 1991). According to Randall and Wolff (1994), the relevance and practical value of a model is limited if it explains a large portion of the variance in smoking in cross-sectional analyses, but only predicts a few percent in smoking in the long run.

In essence, there are two reasons for the confusion about the utility of theoretical models to explain or predict smoking onset. First, cross-sectional analyses do, by nature, overestimate the effects of predictor variables because (a) causes and effects interfere and (b) transitions in use are ignored. With a longitudinal design, more definite conclusions can be drawn about the causality of a relationship. The second reason lies in the strategy employed in longitudinal analyses. A commonly applied method is to consider earlier smoking (time 1) as a predictor variable of smoking status at time 2. Only changes in smoking are predicted in this approach. Another, more finely tuned method, is to examine the precursors of the transition from non-smoking to smoking. It should be stressed that the most informative conclusions can be drawn if one studies the predictors of transitions through stages.

\section{Inconsistencies in Self-reports}

Surveys of substance use rely heavily on self-report measures (e.g., Barnea, Rahav \& Teichman, 1987). The reliability and validity of such measures are therefore a matter of importance. Research has focused intensively on strategies and methods to enhance the quality of assessment of actual substance use patterns (Maisto, McKay \& Connors, 1990; Midanik, 1988). In the field of adolescent substance use, less attention has been paid to the reliability of reports on behaviors and events that occurred in the past (Bailey, Flewelling \& Rachal, 1992). Nonetheless, an often applied predictor variable of substance use and abuse is the age of first experimentation (e.g., Newcomb \& Bentler, 1986). The general assumption is that the younger people start to smoke or drink, the more they are at risk for developing substance use patterns at a later age.

In most studies on smoking or drinking, respondents are asked to provide information about the age at which they first tried a cigarette or took a sip of alcohol. The only way to assess whether people are capable of giving an accurate recall of their history of substance use is in a longitudinal study. When people are asked to provide information at an age close to their actual age of first use, and are then followed for some time, it can be estimated to what extent these data are reliable. One of the few studies focusing on the reliability of age of first use (Bailey et a1., 1992) indicated that only a minority of the adolescents provided consistent. estimates of their first alcohol and marijuana use after a one-year follow-up. This does not give us much hope when respondents are followed for a longer period of time. 


\section{DIFFERENCES IN SMOKING AND DRINKING BEHAVIOR}

"Show me a drunkard who does not smoke, and I will show you a white blackbind" (Green \& Levy, 1976, cited in Johnson \& Jennison, 1992).

Studies on general population, treatment and alcohol-abusing samples have shown that alcohol consumption and cigarette smoking co-occur (see reviews by Bien \& Burge, 1990; Johnson \& Jennison, 1992). In particular, a high prevalence of smoking is observed among alcohol and drug abusers. A study on drug-abusing teens showed that they smoked at rates far and above those of the general population of youngsters (Myers \& Brown, 1994). Jessor and Jessor (1977; Donovan \& Jessor, 1985; Jessor, 1991) argue that there is a constellation of risky behaviors, such as drug use, drinking, smoking, sexual intercourse and truancy which belong to a certain life-style. This is exemplified by prospective analyses which showed that drug use initiated alcohol and cigarette use later on. On the other hand, Bailey's (1992) research on multi-substance use patterns demonstrated that a history of licit use proceeded heavier use and illicit drug use. Although one can debate about the exact ordering of substances, it is possible that different substances are indicators of one general construct.

Although the above mentioned studies emphasize the relevance of focusing on the association between smoking and drinking, there are arguments against this approach. First, although the use of both substances increases dramatically during adolescence, the endpoints are quite different. About $40 \%$ of teenagers smoke whereas about $85 \%$ consume alcohol (De Zwart, Zwart \& Kuipers, 1997; STIVORO, 1997). So one could expect that those who smoke also drink but not always vice versa. A more important point of concern are the norms rellating to these behaviors. The fact that the majority of teens drink implies that for some agegroups drinking is almost normative. This might also count for smoking in subcultures, but not for the general adolescent population. In addition, the widespread use of alcohol in the Netherlands implies that drinking does not always lead to illicit drug use. Another, related issue, is the differences in context in which youngsters start to use alcohol and cigarettes. Drinking a few glasses of wine at dinner or a few pints of beer in front of the television is considered to be quite normal behavior in many households. Many youngsters have their first drink at home with their parents. However, the opposite is true for smoking. Most youngsters start smoking with friends without parental supervision. Of course, these differences in patterns of uptake reflect variations in norms and values. In sum, the different prevalences, situational characteristics, normative beliefs and negative consequences associated with drinking and smoking suggest that different processes could affect changes in these substances and, therefore, should be approached and handled separately. 


\section{OUTLINE OF THIS THESIS}

Chapter 2 introduces the five-year three-wave cohort study from which data for all chapters were derived. General background information about the design, procedure and subjects of the cohort study are provided. This chapter also describes the development of smoking and drinking behavior on the aggregate and individual lewel.

Chapter 3 presents a longitudinal analysis of influence and selection processes in peer groups. More specifically, this study examines the extent to which homogeneity of smoking within immediate peer groups can be ascribed to either adoptation, selection or deselection processes. Chapter 4 includes influence as well as selection processes in a test of the relative impact of peer and parental smoking and drinking behavior on individual use. A structural model was developed and tested, using data from all three waves. The incorporation of cross-lagged links between peer and subject use as well as earlier use by referent persons and subjects, enhances the understanding of a social influence approach.

The associations between alcohol use and the development of intimate relationships are discussed in Chapter 5. This chapter forms a link between Chapters 3 and 4 and Chapters 6 and 7 . It comprises a test of the impact of a steady partner on changes in alcohol use between mid- and late adolescence. On the other hand, it points to the function of drinking in young people's efforts to approach the opposite sex and to develop an intimate relationship. Chapter 6 presents a study on the possible beneficial functions of pub-going for adolescents. Cross-sectional data of the third wave were used to explore the extent pub-going is related to (a) transitions marking the adoptation of more adult roles, (b) appropriate integration in a peer network, and (c) well-being. People might drink to reduce feelings of stress and low self-esteem. Chapter 7 positions the 'stress-reduction hypothesis' in the context of drinking. In particular, drinking at home can not be interpreted as related to integration in peer groups and sociability. Therefore, "home drinkers' might have other reasons for their drinking habits. Drinking as a coping mechanism for feelings of psychological maladjustment could be one of those reasons. Data from the third wave were used to address the research questions.

The aims of Chapter 8 are twofold. First, it examines precursors of the initiation of cigarette smoking. Smoking-specific cognitions, social influences and socio-demographic factors were used as model variables associated with onset. Second, this chapter deals with a methodological issue; namely the extent to which the predictive value of this set of factors is due to the research design and analytic strategy employed. Chapter 9 presents a study on the antecedents of adolescents' motivation to quit smoking. Smoking-specific cognitions, social influences and habitual factors were considered as predictor variables. The stages of change framework of Prochaska and co-workers was used to distinguish smokers according to their degree of motivation to quit. 


\section{DEVELOPMENT OF SMOKING AND DRINKING IN A COHORT OF SECONDARY SCHOOL CHILDREN ${ }^{1}$}

Adolescence is a period in which people are confronted with potentially risky behaviors, such as smoking, alcohol and illicit drug use. Although for some youngsters cigarette use is a temporary phenomenon, many of them take up regular smoking in their teenage years, as is shown by the prevallence of smoking in the United States (US Department of Health and Human Services, 1997) and in European countries (European Bureau for Action on Smoking Prevention, 1994) in young people. In early adolescence, between the ages 9 and 12 , there is a peak in the period of experimentation. The large numbers of daily smokers are established between middle and late adolescence (ages 16 to 20). National surveys on alcohol use in the US have reported that levels of consumption increasing rapidly during the secondary school years (Grant et al., 1988; Johnston, O'Malley \& Bachman, 1991). A crossnational study supervised by the Permanent Observatory on Youth and Alcohol (1994) in 18 European countries showed that starting to drink is 'normal' for the vast majority of European youth.

The development of smoking and drinking in the Netherlands is comparable to other Western countries. A recent study among a representative sample of Dutch secondary school children showed a marked increase in cigarette use (De Zwart, Stam \& Kuipers, 1997). Life-time prevalence increased from 20\% (11-12-years olds) to 66\% $(18+)$. The prevalence of regular smoking (i.e., at least once in the past four weeks) increased from $3 \%$ for the 11 to 12 year-olds to $39 \%$ for the $18+$ category. The main gender differences in smoking concerned the quantity of use: boys smoked more cigarettes per day. The trends of initiation were rather similar for alcohol use. Although $44 \%$ of the 11 to 12 year-olds had never used alcohol, only $10 \%$ of the 
youngsters above 15 reported total abstinence. The figures were even more striking for the increase in frequency of drinking. Only $0.5 \%$ of the $11-12$-year olds reported having drunk alcohol more than 10 times in the last four weeks while this percentage was 33 for the $18+$ category. The gender differences became more explicit with age: boys drank more frequently, consumed more glasses per occasion and reported drunkenness more often than girls. In this chapter, information is provided on the development of adolescent smoking and drinking on the aggregate and individual level.

\section{Continuity in Substance Use on the Individual Level}

Prevalence-data display how drinking and smoking behavior is distributed over the reenage years (or, in the present research, over the five years of the study). Nevertheless, prevalences tell us very little about the continuity or discontinuity of these behaviors. For this purpose, information about the changes in substance use should be analyzed not on the aggregate level but on the individual level.

Studies on the natural history of adolescent alcohol use are highly inconclusive about the stability and predictability of drinking habits over time. Using correlational analyses, Newcomb and Bentler (1989) reported a relatively high correlation $(r=.53)$ of the frequency of drinking over time. However, other researchers, computing correlations of consumption levels at different time-points (Plant et al., 1985) or using transition matrices to examine developments through categories of consumption level (Grant et al., 1988; Temple \& Fillmore, 1985-1986), showed rather low predictive value of earlier drinking on future use. The main impression is that adolescent drinking is a significant but weak predictor of future drinking habits (e.g., Fillmore, 1988). In the field of smoking, it is generally assumed that smoking acquisition is a gradual process in which cigarette use in childhood leads to a higher risk of regular smoking at later ages. Nonetheless, only a few longitudinal studies addressed this issue. An exception is a study by Chassin et al. (1990) following youngsters from their adolescent years into young adulthood (their $20^{\circ}$ s) showing the strong impact of different smoking levels on regular use later on.

According to Pape and Hammer (1996), inconsistent findings may reflect shortcomings in (a) the assessment of variables and (b) differences in statistical analyses. For instance, it is well known that single indicators of behavior have a component of measurement error too high to show a degree of stability (Epstein, 1979). Employment of different indicators of the same construct or behavior tend to increase the stability coefficients. Furthermore, incorporation of abstainers in analyses on consumption measures, a category which is relatively consistent in behavior over time, might lead to overestimation of behavioral continuity. Thus, analyses that exclude abstainers might result in lower consistency coefficients. In addition, it is quite reasonable that stability in categorized variables (as frequency is assessed in most studies), with mostly small wariance in scores is, in general, higher 
than the stability of variables measured on a ratio scale (like quantity of use) with a large variation in scores in adolescent samples.

Some researchers have stressed that becoming a smoker is a developmental process (Hirschman et al., 1984; Leventhal \& Cleary, 1980; Prochaska et al., 1992; Stern, Prochaska, Velicer \& Elder, 1987; Werch \& Anzalone, 1995). First, there is a preparatory stage during which attitudes are formed and modified. Next, there is an experimental stage during which people try their first cigarettes. The experience of these acts, and the reactions of important others in the immediate surroundings, affect whether people proceed with smoking or not. If an individual smokes in a variety of situations, with different types of audience, and more intensively during the day, s/he is more likely to become an addicted regular smoker. Although there might be several paths through these stages, Hirschman and co-workers (1984) suggested that, for the mainstream of youngsters, moving from one stage to another takes about two years. An assumption of this 'smoking career' approach is that, in terms of probabilities, experimental smokers have a higher chance of becoming regular smokers than nonsmokers. Murray, Swan, Bewley and Johnson's prospective study (1983) among junior high school students showed that experimental smokers had a likelihood of .44 of becoming regular smokers compared to .08 of the never-smokers. Unfortunately, few prospective studies have been concentrating on this stage-approach. On the basis of the findings available, it is quite difficult to draw any conclusions about its value (Werch et al., 1995). One of the reasons for the lack of studies is the expensive nature of this type of research, in which youngsters must be tracked and interviewed several times during their adolescent years. Nonetheless, it is an essential requisite for prevention programs focusing on youngsters just before or during their preparation or experimental stage to know if, for instance, experimental smoking leads to regular smoking (Bruvold, 1993). If many early adolescent smokers mature out and quit smoking, or if adult smokers came equally from both adolescent smokers and nonsmokers, prevention programs might be ineffective in prevention of late adolescent or young adult outcomes (Chassin, Presson, Sherman \& Edwards, 1990). The relevance for alcohol prevention programms of scrutinizing whether experimental drinking in early adolescence affects drinking in late adolescence is less clear. Since cross-sectional studies have shown that drinking per se becomes almost the norm for the bulk of the population, the transition from moderate drinking to heavy drinking or problem. drinking in particular is probably the most relevant one.

An important remark must be made with respect to a test of a stage transition approach. Although, in terms of probabilities, there might be a relatively low chance that a never-smoker becomes a regular smoker, the size of the never-smoking group should not be neglected. Chances should be related to the actual size of a group. For example, the data of Murray and colleagues (1983) also showed that the highest contribution to the growing prevalence of regular smokers came from the neversmoking group. In the present study, we evaluated our findings in terms of likelihood (e.g., what is the chance that a never-drinker becomes a weekly drinker) as well as in 
terms of prevalence and incidence (e.g., how many youngsters smoke or drink at a specific wave and what was their former behavior).

To summarize, two analytic strategies in longitudinal research are mainly employed to examine the continuity of substance use over time, namely (a) correlational methods on discrete and interval data comparing smoking and drinking status and (b) transitional matrices on discrete data comparing absolute status (for a combination of both methods, see Pape \& Hammer, 1996a). In this chapter, the outline of the study by Pape and Hammer has been followed. Correlational methods on stages and consumption levels were employed to examine the relative position over time. In addition, transition tables were used to make the development from one state to another visible (c.f. Skog \& Duckert, 1993). For smoking, five states were constructed based on behavioral aspects (see Goddard, 1990). Data on substancespecific cognitions, such as intentions or pro-smoking attitudes, were not included in the classification. Additionally, four states of drinking were constructed. For drinking, the focus was not only on the acquisition process in terms of frequency of drinking, but also in terms of the consumption levels. It is relevant to mention that all measures of drinking and smoking consist of multiple items in order to minimize flaws in estimation due to measurement errors. As shown in the Method section of this chapter, the number of items to construct a variable ranges from 2 to 4 .

\section{Situational Aspects of Substance Use}

The ways of starting may be different for smoking and drinking. First, teenagers often report that their first alcoholic drink was offered by parents in the family home (Wilks and Callan, 1987). The opposite is true for smoking. According to the qualitative work of Presti, Ary and Lichtenstein (1992) and Friedman, Lichtenstein and Biglan (1985), most smokers tried their first cigarette in the company of peers. During adolescence, youth is spending more of its leisure time in settings outside the parental home. This pattern is reflected in the context of use. In mid-adolescence, drinking is usually concentrated at parties and in public drinking places within a network of peers and without the supervision of parents (e.g., Engels, Knibbe \& Drop, 1998a; Harford \& Grant, 1987; Thombs \& Beck, 1994). Although drinking is restricted to certain settings (i.e., youngsters usually do not drink at school or on the street), and smoking is socially tolerated in more settings, smoking and drinking meet each other in social situations, like informal parties, bars, discos, and special evenings with friends (see also Johnson $\&$ Jennison, 1992).

The situations young people smoke or drink in are related to normarive expectations associated with the particular behavior. The majority of parents do not object to drinking to celebrate an anniversary or when going out with friends. However, drinking solitarily or drinking for non-social reasons, such as to relieve stress, is considered to be inappropriate (Wilks et al., 1988). In the case of smoking, one of the last steps in the acquisition process is, in many cases, smoking in the 
presence of parents. Despite the fact that many parents smoke themselves, many youngsters are not allowed to smoke at home. In sum, variations in opinions and norms are reflected in the situations in which people drink and/or smoke.

Situational preferences could not only be age-related but also be related to the duration of the habit. For cigarette use particularly, long-term smoking with its addictive nature may lead to the 'necessity' to smoke more intensively during the day and eventually to smoking in more situations (Hirschman et al., 1984, Shiffman, 1988). To withdraw from smoking in the evening, for instance, becomes more difficult for addicted youngsters who smoke at school-hours and after school-hours but not at home. It is therefore expected that those who smoke or drink for many years, are doing so not only in more situations but also in different situations from beginners.

\section{The Present Study}

The main objectives of this chapter are (a) to provide information about the design, procedure and subjects of the cohort study and (b) to describe the development of smoking and drinking behavior. Although there are more representative data available on adolescent substance use in the Netherlands, changes on the aggregate level are presented by two reasons. First, because different subsamples were used in some of the chapters, it is not easy to get a clear view on the smoking and drinking trends in this study. Second, it permits us to examine whether our findings resemble those of other Dutch surveys. On the individual level, different analytic approaches were employed to investigate stability and instability of substance use patterns over time. Furthermore, the development of patterns is described by the changes in the situations in which young people drink and smoke.

\section{METHODS}

\section{Design and Procedure}

The main goal of this thesis is to throw light on processes leading to the onset of smoking and drinking, and the processes leading to changes in these behaviors. To do this, a longitudinal design is necessary. Since the taking up of smoking and drinking takes place primarily in adolescence, it is important to follow a cohort of youngsters from early adolescence (ages 10-13), a period in which the prevalence of smoking and drinking is rather low, into late adolescence (ages 17-20), which is signified by a large number of regular smokers and drinkers (De Zwart et al., 1997; Dutch Foundation on Smoking and Health, 1997; Results-section of this chapter). Therefore, in this study, a sample of youngsters was interviewed three times. The first measurement was at the beginning of their secondary education and the second measurement was carried out two years later when most adolescents were in the third year of their 
education. The third measurement was carried out five years later; a period that characterizes the end of the subjects' secondary school period, the beginning of working life, or the start of a post-secondary school education.

In 1989 (T1), the study started with a sample of students from first and second grade of secondary education in the Netherlands. A selection of 65 schools, differentiated by educational level and region (the provinces in the Netherlands) was approached for participation in the study. From the vocational schools $(n=17)$, three schools refused to participate. The refusal rate for the high schools $(n=48)$ was higher. We did not obtain formal consent from twenty-six school boards. One reason was privacy: names and addresses of subjects were needed for follow-up surveys. Furthermore, participation in other research or intervention programs was a reason for refusal. A sample of 36 schools finally participated.

Some studies have shown that questionnaires designed for both vocational and high schools might lead to problems with filling out the form for the former schools. In a pilot study, two classes of students from vocational schools were asked to fill out the questionnaire. In addition, a personal interview was carried out with a few pupils to trace difficulties. The final questionnaire was constructed guided by the findings of this pilot.

For the first measurement (Autumn 1989), the questionnaires were administered in the classrooms in the presence of a teacher, and the questionnaires were returned in a sealed envelope. No explicit refusals were recorded; non-response was exclusively due to absence (e.g., sickness) at the day of assessment. 1,454 adolescents participated in the first wave. The form took about an hour to complete. In the summer of 1991 (T2), an introductory letter about the procedure of the second wave was sent to the parents and respondents. In the autumn of 1991, forms were sent to subjects' homes. Subjects who did not respond to two mailed reminders were approached by phone to complete an interview. The response rate was $82 \%$ ( $n=$ 1192) in the 1991-follow-up survey.

Before the third wave took place, a second pilot study was carried out. New scales were added to the questionnaire for reasons of maturation of respondents, changes in main activities and social contacts, and new research questions. The revised questionnaire was tested by 43 adolescents aged $17-19$ at the Public Library in Maastricht. They were contacted to fill out the form and to make comments. Afterwards questions were asked about required time, length of the form and complexity of the questions. Furthermore, because of the older age of respondents and expired time after the second measurement (three years), it was assumed that many respondents had moved from their original addresses. Thus, by contacting community registers and checking addresses of parents or respondents by $C D$-phone (registered phonebook on cd-rom) almost all addresses were retrieved. In August 1994, a letter was sent to the addresses of parents or respondents to verify their homes and to introduce the third wave. In the autumn of 1994 (T3), the forms were sent to the participants. Mailed reminders and telephone interviews (CATI-format) were 
used to enhance the response rate. 1187 subjects ( $82 \%$ ) participated at the third wave. In total, data from 1,063 subjects $(73 \%)$ were collected from all three waves.

Attention was drawn to the confidentiality of responses (see Botvin $8 x$ Botvin, 1992). The letters of introduction and the questionnaires emphasized privacy aspects, and clearly stated that no information about the specific responses of subjects would be passed on to teachers or parents. The subjects put the filled out form in a sealed envelope so that the teacher was not able to check their responses. No anonymous questionnaires could be used due to the fact that we matched numbers and subjects" names for the follow-up surveys. Even so, matching of numbers and names was only done by the principal researcher.

One of the major problems conducting longitudinal surveys is to keep the attrition rates low. Response rates of $82 \%$ at the two follow-ups, and a overall response of $73 \%$, is relatively high compared to other longitudinal surveys on substance use by adolescents (Ary \& Biglan, 1988; Bauman, Fisher \& Koch, 1989; Chassin et al., 1984; Flay et al., 1994; Murray et al., 1983; Pederson \& Lefcoe, 1987). ${ }^{2}$ Although it is impossible to assess their impact on the response rates, we assume that an up-to-date address file, the use of reminders and telephone interviews, financial rewards (participants were offered the opportunity of 25 bonus prizes amounting $\$ 20$ (only at T3) and personal attention (those who were interested received a summary of preliminary results of the study (at T3) contributed to high response rates.

\section{Attrition Analyses}

As some studies in this area have shown (e.g., Ary \& Biglan, 1988), adolescents with problem behaviors, such as playing truancy, having a troublesome relationship with parents, using drugs, smoking and drinking, might be less prone to remain involved in a prospective study. On the other hand, there is some indication that people who refrain from alcohol, tobacco or drugs think that the form is irrelevant to them, and are therefore less likely to participate (Arner, Duckert \& Hauge, 1980, cited by Pape, 1997). We assume that this is less applicable to our study since we explicitly mentioned this issue in the introductory letters and the questionnaires.

When there is selective attrition, some caution is warranted in generalizing the findings to the general population or to specific subpopulations. A logistic regression analysis was conducted to compare participating subjects and the drop-outs with data from the first measurement as predictors of drop-out (see for details Chapter 4). Drop-outs $(n=391)$ appeared to be older, less educated, attended a church less often and less often had a father of Dutch origin than remainers $(n=1,063)$. Small differences $(p<10)$ were found for sex and intensity of cigarette smoking. Drop-outs were more likely to be male and reported a higher number of cigarettes smoked than remainers. No differences $(p>$.15) were found for frequency of drinking and smoking, or intensity of drinking. Although there was small but detectable selective 
attrition, no salient differences between respondents and dropouts on relevant behavioral outcome variables could be determined.

\section{Sample Characteristics}

The sample participating at all three waves consisted of 520 girls ( $49 \%$ ) and 543 boys $(51 \%)$ (Table 2.1). At the first wave, the mean age was $12.4(S D=.7)$. About $16 \%$ of the subjects were living in the three northern provinces in the Netherlands (Groningen, Friesland, Drente), $18 \%$ in the central/eastern provinces (Overijsel, Gelderland, Noordoostpolder), 44\% in the western provinces (Noord-Holland, ZuidHolland, Utrecht, Zeeland) and $21 \%$ in the southern provinces (Brabant, Limburg). Most subjects ( $75 \%$ ) were living in municipalities of fewer than 50,000 inhabitants. Thirteen percent lived in municipalities with 50,000 to 100,000 inhabitants and $12 \%$ in municipalities with more than 100,000 inhabitants. In 1989, one-third of the students were enrolled in vocational school and $65 \%$ in high school. A large proportion of the parents, $53 \%$ of the mothers and $49 \%$ of the fathers, had only finished primary or low secondary education. Only $5 \%$ of the mothers and $14 \%$ of the fathers followed any type of post-secondary education. Most youngsters (87\%) lived with two parents (including step-parents). Ninety-three percent had a father or/and mother of Dutch origin. With regard to religious affiliation, $29 \%$ reported having a mother who was Catholic, 29\% Protestant, 4\% Islamic and 5\% some other kind of religion. Only $16 \%$ indicated going to a church once a week or "often'. Furthermore, almost one out of five youngsters reported having a job on the side at the age of 12 .

\section{Measures}

All variables were assessed identically at each wave except for the quantity of alcohol consumption per week (see below).

Cigarette Smoking. A classification of stages of smoking initiation was constructed by asking the following questions. At all waves, subjects were asked to indicate their lifetime smoking: 'Have you ever smoked a cigarette, even a few puffs?'. Furthermore, two questions were used to assess smoking in the last three months and the last four weeks. Response categories for smoking in the last four weeks were 'daily', 'weekly', 'less than weekly" and 'did not smoke in the last four weeks.' These questions were used to construct a smoking index on a 5-point scale; 1 "never smoked', 2 'had smoked, but not in the preceding three months', 3 'had experimented with smoking or smoked regularly, but did not smoke in the past 4 weeks", 4 'smoked occasionally, at least in the past month' and 5 'smoked daily' (WHO, 1983; for a comparable method, see Goddard, 1990). The amount of smoking was measured by adding the number of cigarettes and hand-rolled cigarettes smoked per week. Only subjects reporting smoking in the past four weeks were asked to fill out these questions. The 
Table 2.1. Characteristics of the Sample in 1989

\begin{tabular}{|c|c|c|}
\hline & $\%$ & $\mathbb{N}$ \\
\hline \multicolumn{3}{|l|}{ Gender } \\
\hline boys / girls & $51 / 49$ & $(543 / 520)$ \\
\hline \multicolumn{3}{|l|}{$\mathrm{Age}^{\mathrm{a}}$} \\
\hline$\leq 11 / 12$ & $.3 / 65$ & $(3 / 691)$ \\
\hline $13 / \geq 94$ & $27 / 8$ & $(281 / 86)$ \\
\hline \multicolumn{3}{|l|}{ Region in the Netherlands } \\
\hline north / east & $16 / 18$ & $(167 / 185)$ \\
\hline west / south & $44 / 21$ & $(450 / 217)$ \\
\hline \multicolumn{3}{|l|}{ Urbanization } \\
\hline$<50,000$ habitants & 75 & $(772)$ \\
\hline $50,000-100,000$ habitants & 13 & $(128)$ \\
\hline$>100,000$ habitants & 12 & $(119)$ \\
\hline \multicolumn{3}{|c|}{ Level secondary education respondent } \\
\hline low & 35 & (361) \\
\hline middle & 39 & $(411)$ \\
\hline high & 26 & $(272)$ \\
\hline \multicolumn{3}{|l|}{ Education father / mocher ${ }^{b}$} \\
\hline only primary school & $6 / 10$ & $(35 / 60)$ \\
\hline low secondary education & $47 / 39$ & $(296 / 230)$ \\
\hline middle/high sec. educ. & $43 / 37$ & $(274 / 217)$ \\
\hline post-secondary education & $5 / 14$ & $(32 / 81)$ \\
\hline \multicolumn{3}{|l|}{ Living arrangements } \\
\hline both parents (step-parents) & 87 & $(898)$ \\
\hline one parent & 5 & (53) \\
\hline other & 8 & $(85)$ \\
\hline \multicolumn{3}{|l|}{ Origin } \\
\hline parents are both Dutch & 86 & (915) \\
\hline one parent is Dutch & 7 & $(75)$ \\
\hline neither of the parents is Dutch & 7 & $(73)$ \\
\hline \multicolumn{3}{|l|}{ Religion mother } \\
\hline Catholic / Protestant & $29 / 27$ & $(302 / 286)$ \\
\hline Isllam / other & $4 / 5$ & $(41 / 54)$ \\
\hline no & 33 & (346) \\
\hline \multicolumn{3}{|l|}{ Having a job (on the side) } \\
\hline yes/no & $18 / 82$ & $(189 / 869)$ \\
\hline \multicolumn{3}{|c|}{$\begin{array}{l}\text { For some variables, the absolute number of subjects does not sum up to } 1,063 \text {. This is due to } \\
\text { missing values on that particular item. The percentages are calculated for the available number of } \\
\text { subjects. Besides the questions on parental education, the percentage of missing values does not } \\
\text { exced } 4 \text { for the variables described in the table. "Many youngsters were not aware of the } \\
\text { (precise) educational background of their parents. } 420 \text { subjects explicitly reported that they did } \\
\text { not know the highest education their mother had finished (471 subjects for father's education). } \\
\text { Thus, the percentages depicted in this table should be interpreted with caution. }\end{array}$} \\
\hline
\end{tabular}


same category of smokers was asked whether they smoked in the following seven situations: parental living room or kitchen, own room, parties or special evenings, friends" homes, bars or discos, school and on the street. The responses were on a dichotomous scale: 1 'no', 2 'yes'.

Alcobol Consumption. Subjects' alcohol use was assessed by an index constructed of three questions. For life-time drinking (separately for beer, distilled spirits and other beverages), subjects were asked if they ever drank alcohol, even a sip. In 1994, the previous three years were used as a reference period. In addition, questions were asked about alcohol use in the previous six months and in the previous four weeks. Response categories for drinking in the last four weeks were 'daily', 'weekly", "less than weekly' and 'did not consume alcohol in the last four weeks'. A classification was constructed on a 5-point scale: 1 'total abstainer', 2 "experimental drinker, consumed alcohol in the past 6 months", 3 'occasional drinker, consumed alcohol in the past four weeks' and 4 'regular drinker, used alcohol at least once a week'.

Alcohol consumption in wave one was assessed by asking the number of glasses consumed in the last week. A distinction was made between the weekend days (3) and the weekdays (4). In wave two and three, weekly alcohol consumption was measured by four items. Not only was a distinction made between weekend day and weekdays, but also between two 'situations', namely inside their (parental) home or outside their home (i.e., mostly in public drinking places and at parties). Furthermore, the responses on the frequency of drunkenness were transformed to 1 'not in the preceding 6 months' and 2 'at least once in the preceding 6 months'. In the third wave, the frequency of drinking more than six glasses per occasion was asked, which served as an indicator of heavy drinking. Respondents who reported drinking in the previous four weeks were asked whether they had consumed alcohol in the following seven situations: parental living room or kitchen, own room, parties or special evenings, friends. homes, bars or discos, school and sport canteen.

\section{Strategies for Analyses}

Since previous research showed that the development of drinking and smoking patterns is, to some extent, gender-specific, the main analyses are performed for males and females separately.

Charnges in Srnoking and Drinking on the Aggregate Level. First, for the total sample, we calculated the smoking and drinking status across the three waves. Trends and gender differences were computed by means of cross-tabulations using chi-square statistics. Furthermore, the changes in quantity of cigarette use and alcohol consumption were examined by means of $t$-tests. ${ }^{3}$ In addition, in all three waves, the proportion of subjects being drunk at least once in the six months was computed.

Changes in Smoking and Drinking on the Individual Level. Different approaches were applied to examine whether (a) there is continuity in drinking and smoking during adolescence, and (b) the acquisition of substance use progresses through different 
states. Concerning the first question, Spearman correlations were used to investigate the associations between the states over time. Pearson correlations were applied to examine the associations between drinking and smoking levels over time for those who were occasional or regular users. Analyses were performed on the untransformed variables and the log-transformed variables. In addition, multiple regression analyses were used to scrutinize to what extent heavy drinking and regular smoking at wave three could be predicted by use at wave two. No multivariate analyses with wave one-data were conducted because of the low numbers of drinkers. The analyses predicting substance use at the third wave are most informative because of the strong increase in drinking and smoking between middle and late adolescence.

One-step transition tables were calculated to investigate whether teens' smoking and drinking develop through distinct states. In terms of probabilities, it was investigated how likely it is that someone who, for instance, tried smoking at wave one, stayed in the same state or made a transition to another state. The tables were calculated for the T1-T2 transitions and the T2-T'3 transitions. No separate tables for boys and girls were presented. Possible gender differences are presented in the text. The category of weekly drinkers turned out to be quite heterogenous, in particular in late adolescence (T3) when it includes adolescents who consume one or two glasses a week as well as adolescents who consume more than 15 glasses a week. An additional analysis was therefore carried out on the transitions from consumption levels at wave two to consumption levels at wave three. Notice that no normative cut-off points were used. The classification of drinkers was constructed by dividing subjects who reported drinking in the past four weeks into three categories (e.g., about equal in size and separately for the sexes). This analysis was not done for the T1-T2 transitions by reason of the low number of drinkers at the first wave.

Changes in the Context of Substance Use. The percentages of users in different situations were computed for each wave. The aim of this analysis was to scrutinize the changes in situations due to (a) the rising age of the subjects and (b) differences in duration of substance use. The analyses were conducted only for those who reported smoking or drinking in the preceding four weeks before administration of the questionnaire. For both drinking and smoking, 'use' was defined as drinking (or smoking) at least once in the preceding four weeks. In general, analyses were performed for the subsample of users at each particular wave. Additional analyses were carried out comparing subjects who started drinking or smoking at wave three with subjects who also drunk or smoked at the preceding wave.

\section{RESULTS}

\section{Changes in Smoking and Drinking on the Aggregate Level}

The prevalence of smoking increases rapidly during adolescence (Table 2.2). While at the first wave the majority of subjects never even tried a cigarette, only one fourth of 
the sample reported not having smoked at the third wave. Additionally, the percentage of daily smokers in the total sample increased from 3 at the first wave, through 10 at the second wave to 31 at the third wave. There were some gender differences in these trends. More girls than boys experimented with smoking in 1989 $(4,1051)=10.66, p<.05)$ while there were more regular smokers among girls in $1991(4,1029)=19.23, p<.001)$. In 1994, more girls smoked on an occasional basis $(4,1024)=12.55, p<.05)$. Furthermore, large differences were found for the amount of cigarette use across the waves. Both boys and girls smoked more cigarettes per week over the years. Nonetheless, boys smoked significantly more $(p<.01)$ cigarettes per week than girls at wave two and three.

Table 2.2. State of Smoking and Quantity of Smoking at Three Waves

\begin{tabular}{|c|c|c|c|}
\hline Mean age & $\begin{array}{c}\mathrm{T} 1(1989) \\
12.4 \\
\end{array}$ & $\begin{array}{c}\mathrm{T} 2(1991) \\
14.4\end{array}$ & $\begin{array}{c}\text { T3 }(1994) \\
17.4\end{array}$ \\
\hline \multicolumn{4}{|c|}{ Smoking states (\% girls in parrentheses) } \\
\hline has never smoked & $57 \quad(65)$ & $(41)$ & $(28)$ \\
\hline tried smoking & $(20)$ & $(29)$ & $(25)$ \\
\hline used to smokse & (3) & $(6)$ & $(3)$ \\
\hline smokes occasionally & $(10)$ & (13) & (16) \\
\hline smokes daily & (2) & (11) & $(29)$ \\
\hline \multicolumn{4}{|c|}{ Intensity of smoking (no. cigarettes per week) } \\
\hline Boys & $13.2(19.3)^{b}$ & $29.3 \quad(30.5)$ & $68.2(49.5)$ \\
\hline Girls & $8.8(19.9)$ & $18.0 \quad(21.3)$ & $53.2(41.9)$ \\
\hline
\end{tabular}

${ }^{2}$ Only for occasional and daily smokers ${ }^{\mathrm{b}} \mathrm{SD}$ in brackets

Table 2.3 depicts the changes in alcohol use. While at the ages of 12 and 13 about one third never drank alcohol, only $5 \%$ reported abstaining five years later. The increase in regular (i.e., weekly) drinking was most prominent between wave two and three. Boys drank more frequently than girls - significant $(p<.001)$ gender differences in allocation to the states were apparent at all waves. Furthermore, the adolescents drank not only more frequently but also more intensively at the third wave.The rapid increase in alcohol consumption, especially between wave two and three, is caused by the increase of drinking in public drinking places.

In general, boys drank more glasses per week than girls. Although no differences in use were found for data of the first wave, significant $(p<.01)$ differences in consumption levels between boys and girls were displayed at the second and third wave. An analysis on the changes in frequency of drunkenness revealed that between wave two and three the percentage of youngsters reporting drunkenness at least once in the past six months increased with a factor ten. At wave three, one fifth of the males reported being drunk at least once a month. 
Table 2.3. State of Drinking, Quantity of Drinking and Drunkenness at Three Waves

\begin{tabular}{|c|c|c|c|c|c|c|}
\hline Mean age & \multicolumn{2}{|c|}{$\mathrm{T} 1(1989)$} & \multicolumn{2}{|c|}{ T2(1991) } & \multicolumn{2}{|c|}{ T3(1994) } \\
\hline \multicolumn{7}{|c|}{ Drinking states (\% girls in parentheses) } \\
\hline has never drunk & 31 & (43) & 15 & $(18)$ & 4 & (6) \\
\hline tried drinking & & $(44)$ & 46 & $(45)$ & 15 & (23) \\
\hline drinks occasionally & 16 & (12) & 26 & $(31)$ & 19 & (33) \\
\hline drinks weekly & 5 & (1) & 13 & $(6)$ & 62 & (38) \\
\hline \multicolumn{7}{|c|}{ Consumprion levels (no. glasses per week) } \\
\hline \multicolumn{7}{|c|}{ Total } \\
\hline Boys & 2.8 & $(6.3)^{\mathrm{b}}$ & 5.8 & $(9.3)$ & 14.3 & $(17.6)$ \\
\hline Girls & $\mathbb{1 . 8}$ & $(3.4)$ & 2.7 & $(5.0)$ & 5.2 & $(6.6)$ \\
\hline \multicolumn{7}{|l|}{ At home } \\
\hline Boys & & & 1.5 & (3.6) & 3.3 & $(6.9)$ \\
\hline Girls & & & .7 & (2.1) & .8 & $(2.0)$ \\
\hline \multicolumn{7}{|c|}{ At public drinking places } \\
\hline Boys & & & 4.3 & (7.3) & 11.0 & $(13.6)$ \\
\hline Girls & & & 2.0 & $(3.8)$ & 4.4 & $(5.7)$ \\
\hline \multicolumn{7}{|c|}{ Drunkenness (once per 6 months, $\%$ ) } \\
\hline Boys & 3 & & 4 & & 49 & $(22)^{\mathrm{c}}$ \\
\hline Girls & 1 & & 3 & & 36 & $(8)$ \\
\hline
\end{tabular}

${ }^{2}$ Only for occasional and weekly drinkers. ${ }^{b} S D$ in brackets. ${ }^{c}$ Percentage of adolescents that reported drunkenness at least once a month in brackets

\section{Stability of Smoking and Drinking Behavior}

A first view on the stability of smoking and drinking behavior was carried out by computing correlations over time (Table 2.4). First, the correlations between the smoking states over time were relatively high, ranging from .41 to .71 . This implies that, to a large extent, the smoking status at a given time-point can be predicted by the smoking status at a preceding wave. However, when the amount of smoking was examined, the size of the associations appeared to be low to moderate. One of the explanations for the dissimilarity in findings is that non-smokers are excluded from the latter analyses. It is evident that incorporation of this category, due to its relative stability over time, would increase the magnitude of the associations. Additionally, individual responses on the two measures employed were quite different in nature. It was more likely that people reported a similar state of smoking than a similar number of cigarettes at a subsequent wave. In general, the associations for alcohol use over time were lower than for smoking. Correlations of the states of drinking over time were higher than the correlations between the levels of consumption. There were some gender differences for the drinking levels - the levels of girls could hardly be predicted by those measured at an earlier wave (except drinking at 'T3 by use at T2). 
Table 2.4. Stability of States of Smoking and Drinking, and Quantity of Cigarette and Alcohol Use across the Three Waves

\begin{tabular}{|c|c|c|c|c|}
\hline Time-interval & $\begin{array}{l}\mathrm{T} 1-\mathrm{T} 2 \\
2 \text { years }\end{array}$ & $\begin{array}{l}\mathrm{T} 2-\mathrm{T} 3 \\
3 \text { years }\end{array}$ & & $\begin{array}{l}T 1-T 3 \\
5 \text { years }\end{array}$ \\
\hline \multicolumn{5}{|c|}{ States of smoking (Spearman's sho) } \\
\hline Boys & .69 & 71 & & .53 \\
\hline Girls & .61 & .67 & & .41 \\
\hline \multicolumn{5}{|c|}{ Quantity of smoking (Pearson's $)^{\text {s }}$} \\
\hline Boys $(62 / 95)$ & $.25^{2 t} \quad\left(.32^{*}\right)$ & $.23 *$ & $\left(.21^{*}\right)$ & $.45 \quad(.43)$ \\
\hline Girls $(60 / 120)$ & $.46 \quad(.42)$ & .33 & $(.28)$ & $.14 \pi s\left(.25^{* x}\right)$ \\
\hline \multicolumn{5}{|c|}{ Sitates of drinking (Spearman's rho) } \\
\hline Boys & .50 & .34 & & .23 \\
\hline Girls & .51 & .37 & & .27 \\
\hline \multicolumn{5}{|c|}{ Quantity of drinking (Pearson's $r)^{b}$} \\
\hline Boys $(98 / 170)^{\mathrm{c}}$ & $.23^{+}(.19+)$ & .46 & $(.28)$ & $.21 *(.18 t)$ \\
\hline Givls $(59 / 174)$ & $.11 \mathrm{~ns}\left(.27^{*}\right)$ & .30 & $\left(24^{*+4}\right)$ & $-.10 \mathrm{~ns}(-.14 \mathrm{~ns})$ \\
\hline
\end{tabular}

Note. All coefficients are significant at the $p<.001$-level except $+p<.10, * p<.05, * p<.01$ and $n s p \geq .10$. Only for occasional and daily smokers; correllations with Jog-transformed variables in parentheses. "Only for occasional and weekly drinkers; correlations with logtransformed variables in parentheses. "Because the telephone interview at the third wave did not consists of questions on quantity of drinking, these analyses were performed on respondents who filled out the questionnaire.

To examine the stability of behavioral states in more detail, one-step transition matrices for the total sample were computed. Table 2.5 illustrates that never smokers and daily smokers were the most stable. More changes were observed among those who experimented with smoking or smoked but quit. The least stable were the accasional smokers - almost half this category had returned to the non-smoking state and one third to daily smoking by the second wave, while $60 \%$ smoked daily by the third wave. With regard to drinking states, relatively high stability was observed between $T 1$ and $T 2$, and more instability between $T 2$ and T3 (Table 2.6). The instability between mid and late adolescence could be explained by the fact that most people started to drink weekly. An analysis differentiating weekly and occasional drinkers by their drinking levels showed low stability. In particular, for the low and moderate levels of consumption, no clear trends to one direction or the other could be detected. Those with relatively high consumption levels, however, were relatively stable - almost $60 \%$ stayed in the same category. Thus, an approach in which transitions through behavioral states were computed revealed that smoking and drinking habits are less stable than was indicated by the simple correlations.

A second feature of a "transition approach" is that we can explore whether the acquisition of substance use progresses through distinct states. One of the assumptions was that the likelihood of having the status of, for example, a daily smoker at wave two is linearly related to the allocation of the states at wave one. This 
seems to be the case. The probability of being a daily smoker at wave two was three times higher for those who tried smoking than those who smoked occasionally at wave one. The figures were rather similar for smoking and drinking. Nevertheless, it should be stressed that, although in terms of probabilities those used to smoking were more likely to smoke daily three years later (T2-T3), many more experimental smokers started to smoke daily according to the absolute numbers. A similar picture was observed for weekly drinking between T2 and T3.

Table 2.5. One-step Transition Matrixes for Smoking States in 1989-1991 and 1991-1994 (turnover points are printed in italic; $\mathrm{N}$ in parentheses)

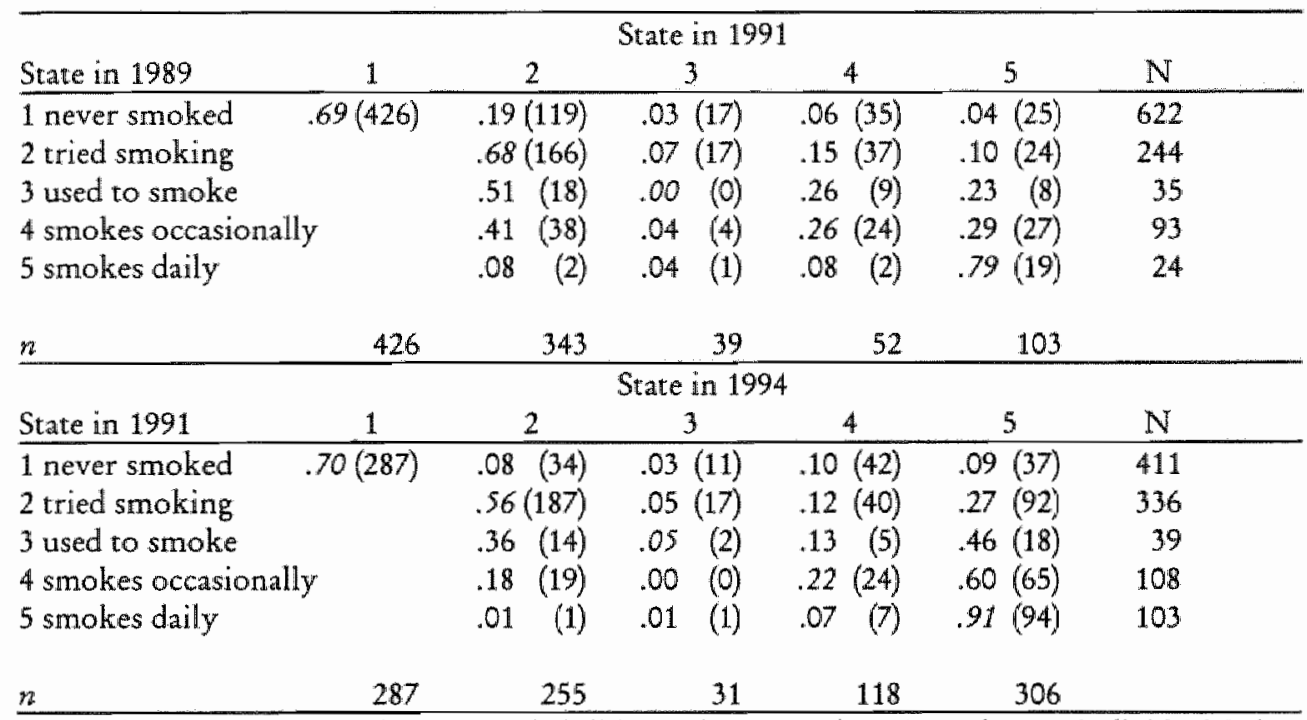

Note. The figures are conditional probabilities: this means that given that an individual is in a certain state at one measurement, the figures show the probability distribution for them occupying each of the possible states at the next measurement.

Multiple regression analyses were conducted to explore whether heavy drinking and regular smoking at T3 can be predicted by indicators of drinking (quantity of use and state of acquisition) within the category of users at T2. These findings (not in the Tables) showed that only a few percent of the variance in the frequency of drunkenness, the frequency of $6+$ drinking and overall consumption levels can be explained by the T2-drinking variables. In five out of six analyses, explained variances did not exceed ren percent. An exception is the frequency of $6+$ drinking among boys; the levels of alcohol use and state of acquisition predicted $6+$ drinking moderately $(23 \%)$. The findings for smoking are rather similar. The quantity 
of cigarettes and the state of acquisition could only predict a few percent (between 6 and $13 \%$ explained variance) of the frequency and quantity of use three years later.

Table 2.6. One-step Transition Matrixes for Drinking States in 1989-1991 and 1991-1994, and for Consumption Levels in 1991-1994 (turnover points in italic)

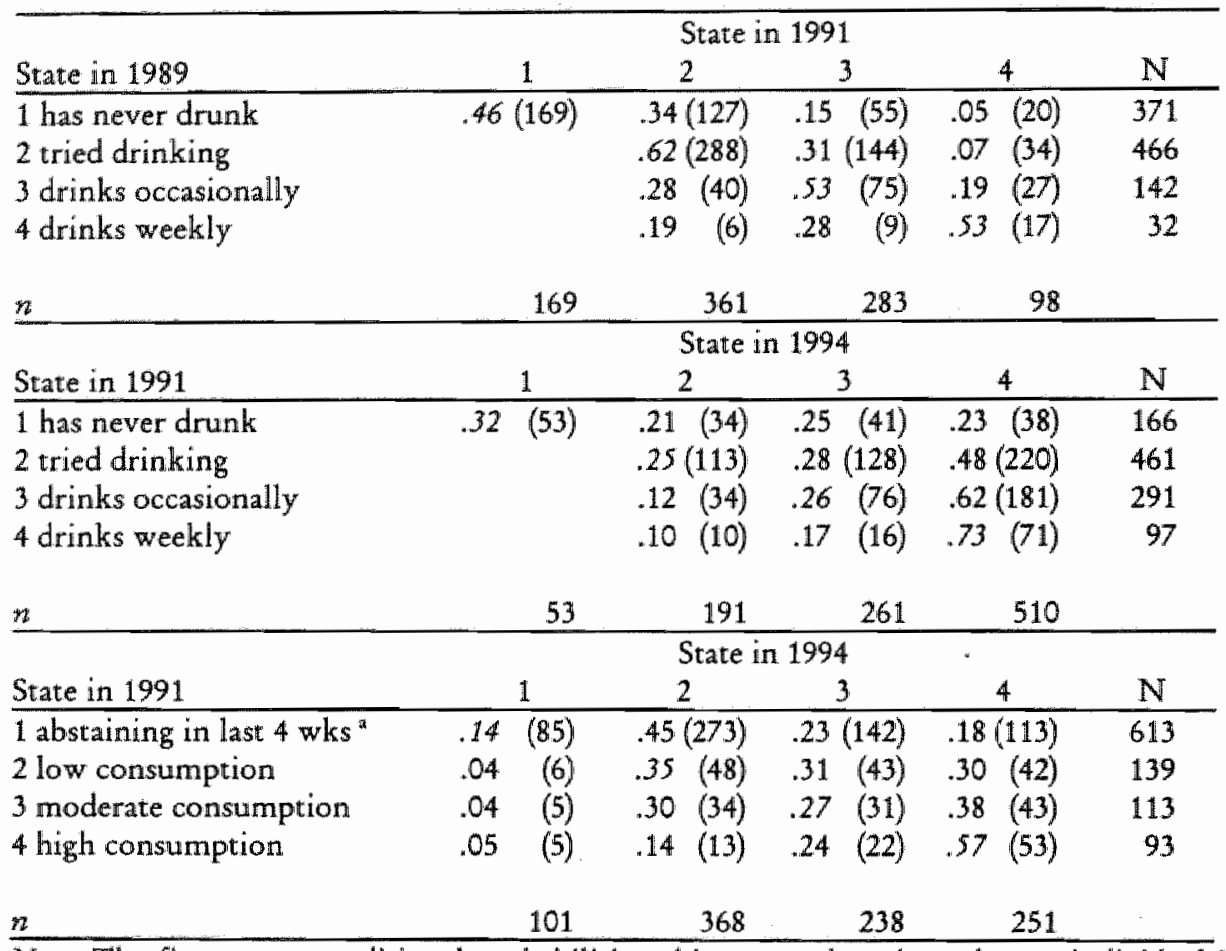

Note. The figures are conditional probabilities: this means that given that an individual is in a certain state at one measurement, the figures show the probability distribution for them accupying each of the possible states at the next measurement. ${ }^{a}$ Because the telephone interview at the third wave did not consist of questions on quantity of drinking, these analyses were performed on respondents who filled out the questionnaire $(n=958)$.

\section{Changes in the Context of Use}

The prevalences of smoking and drinking in seven situations are depicted in Table 2.7. For alcohol use, the number of youngsters drinking in peer settings increased during adolescence. The highest prevalence of drinking was at the subjects' parental home at the first wave. Throughout the years, drinking at parties, special evenings and in public drinking places became more relevant. Not surprisingly, the analyses by gender indicated that boys drank in more settings (especially at T2 and T3). 
Nevertheless, in terms of ranks of 'popular' settings or places, no explicit differences in preferences for specific situations were shown. The findings are different for smoking. In early adolescence, people did not smoke at home but on the street and at school. Over the years, people smoked more frequently at home, in public drinking places and with friends. Only a few gender differences were found, indicating that boys and girls smoked in the same settings.

Table 2.7. Percentage of Drinkers and Smokers (at least once in the past four weeks) in Different Settings across the Waves ( $N$ in parentheses).

\begin{tabular}{|c|c|c|c|c|c|c|}
\hline & \multicolumn{2}{|c|}{1989} & \multicolumn{2}{|c|}{1991} & \multicolumn{2}{|c|}{$1994^{b}$} \\
\hline & $\begin{array}{l}\text { Boys } \\
(114)\end{array}$ & $\begin{array}{c}\text { Girls } \\
(66)\end{array}$ & $\begin{array}{l}\text { Boys } \\
(202)\end{array}$ & $\begin{array}{l}\text { Girls } \\
(190)\end{array}$ & $\begin{array}{l}\text { Boys } \\
(378)\end{array}$ & $\begin{array}{l}\text { Girls } \\
(337)\end{array}$ \\
\hline \multicolumn{7}{|l|}{ Alcobol use } \\
\hline Living room/kitchen & 75 & 67 & 51 & $40^{2+3}$ & 75 & 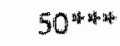 \\
\hline Own room & 4 & 3 & 7 & $2^{\text {虾 呫 }}$ & 19 & 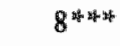 \\
\hline Parties/special evenings & 55 & 63 & 72 & 77 & 91 & 88 \\
\hline Friends' homes & 16 & 22 & 40 & $26^{44}$ & 71 & $48^{24}+25$ \\
\hline Bars/discos & 30 & 24 & 60 & $711^{4}$ & 91 & 92 \\
\hline School ${ }^{2}$ & 1 & 5 & 4 & 1 & 14 & 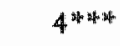 \\
\hline \multirow[t]{2}{*}{ Sport canteen } & 15 & $3^{404}$ & 16 & 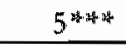 & 30 & $8^{\text {此此地 }}$ \\
\hline & $\begin{array}{c}\text { Boys } \\
(62)\end{array}$ & $\begin{array}{l}\text { Girls } \\
(60)\end{array}$ & $\begin{array}{c}\text { Boys } \\
(95)\end{array}$ & $\begin{array}{l}\text { Girls } \\
(120)\end{array}$ & $\begin{array}{l}\text { Boys } \\
(216)\end{array}$ & $\begin{array}{l}\text { Girls } \\
(223)\end{array}$ \\
\hline \multicolumn{7}{|l|}{ Cigarette smoking } \\
\hline Liwing room/kitchen & 21 & 10 & 35 & 28 & 48 & 53 \\
\hline Own room & 13 & 8 & 32 & 23 & 32 & 28 \\
\hline Parties/special evenings & 19 & 23 & 73 & 71 & 69 & 76 \\
\hline Friends' homes & 23 & 30 & 56 & $42^{\text {th }}$ & 63 & 62 \\
\hline Bars/discos & 27 & 22 & 60 & 63 & 69 & $80^{4}$ 내에 \\
\hline School ${ }^{2}$ & 50 & 43 & 68 & 77 & 69 & 72 \\
\hline Street & 69 & 67 & 75 & 72 & 66 & $56^{* 4}$ \\
\hline
\end{tabular}

Note. The asterisks refer to p-values of chi-square tests of possible gender differences. ${ }^{*} p<.05$, wh $p<.01,{ }^{*} p<.001 .{ }^{*}$ This item referred to use at school and the working place at T3.

- Because the telephone interview at T3 did not contain questions on quantity of drinking, these analyses were performed on respondents who filled out the questionnaire.

Additional analyses were conducted for the seven situations comparing subjects starting to use cigarettes or alcohol at a particular wave with subjects' continuing use over the waves. With respect to drinking at wave two, more continuing consumers than 'starters' reported drinking at home ( 52 ws. $41, p<.05$ ), in their own room ( 9 vs. $2, p<.01)$, at friends" homes ( 41 vs. $30, p<.05)$, at school $(6$ vs. $1, p<.01)$ and in sport canteens $(16$ vs $8, p<.01)$. At the third wave, more continuing consumers that starters reported drinking at friends' homes $(66 \mathrm{vs} .56, p<.05)$ and in public drinking 
places ( 96 vs. $89, p<.001$ ). With regard to smoking at wave two, more continuing smokers than starters used cigarettes at home ( 46 vs. $24, p<.001$ ), in their own room (35 vs. $22, p<.05)$ and at friends ${ }^{5}$ homes $(67$ vs. $39, p<.001)$. Similar findings were revealed for the third wave - differences were found for smoking at home $(60 \%$ of the smokers vs. $43 \%$ of the new smokers, $p<.001)$, in their own room (38 vs. $24, p<$ $.01)$ and on the street $(67$ vs. $56, p<.01)$. No significant differences were found for either smoking or drinking in public drinking places and parties between initial and continuing users.

In sum, data on drinking and smoking in various settings revealed that (a) drinking and smoking patterns are somewhat dissimilar in early adolescence adolescents drink with their parents and smoke with their peers - but converge at later ages, and (b) impact of habit formation is most explicit for smoking behavior. When people smoke for several years, it becomes more integrated into their daily life, with use not restricted to peer settings (such as school, public drinking places or parties).

\section{DISCUSSION}

This chapter aims (a) to provide information about the design, procedure and subjects in the cohort study and (b) to gain insight in the development of smoking and drinking in Dutch adolescents on the individual and aggregate level. When we sum up the findings on the prevalences of smoking and drinking in different stages of adolescence, some aspects are worth mentioning. Firstly, although the number of regular users is relatively small in early adolescence, the number of experimental smokers and drinkers is substantial. This picture alters throughout the years with large numbers of regular smokers ( $31 \%)$ and drinkers $(50 \%)$ in late adolescence. The increase in frequency and intensity of drinking and smoking is most salient between mid and late adolescence. Second, there are marked gender differences. In general, boys drink more frequently, consume more glasses per occasion and report more frequent moments of drunkenness. The case is less clear for smoking. No significant differences were shown between boys and girls for the states of smoking. Nonetheless, boys smoked more intensively than girls at the last two waves.

The Trimbos Institute carries out a large survey on smoking, drinking and drug use of Dutch school children every four years. When the prevalence figures in the current study are compared with the Trimbos data gathered in 1992 (Kuipers, Mensink \& De Zwart, 1993) and 1996 (De Zwart et al., 1997) ${ }^{4}$, the percentages of regular smoking and drinking in early adolescence (11-12 year olds) are rather similar. However, there were some differences in the older age categories; regular smoking and drinking were more prevalent in the present study than in the Trimbos study. An explanation for this difference is that our study comprised youth who followed secondary vocational education, dropped out from school, and worked (or were unemployed) whereas the Trumbos-study included only students of vocational and 
high schools. A study carried out by the Dutch Foundation of Smoking and Health (1997) among a more representative sample of youngsters showed higher rates of regular smoking than the Trimbos-study. Other explanations are related to differences in procedure of administration of questionnaires, the order of the questions, type of (other) variables in the form, exact wording of questions, etc.

\section{Stability or Change in Smoking and Drinking?}

Pape and Hammer (1996) stated that the answers to these questions largely depend on what kind of data and analytic strategy were used. The associations of the frequency measures at different time-points are indeed promising. The correllations range from .41 to .71 for smoking and from .23 to .51 for drinking. However, these analyses include abstainers; a group with relatively stable behavior over time.

The consistency estimates of the quantity measures are much lower due to (a) the exclusion of abstainers and (b) the higher ranges in the responses. Concerning the latter it is important to stress than the scores on a frequency measure with anchors 1 to 5 are, by nature, more stable that the scores on a quantity scale as was used in the current study, with scores ranging from 0 to $\infty$ (i.e., ratio scale). Summarizing, the more precisely one measures alcohol consumption or cigarette smoking, the less stable and predictable the behavior appears to be (see also Bailey, Flewelling $8 x$ Rachal, 1992). Furthermore, the higher correlations between quantity of drinking at wave two and three compared to the correlations between use at wave one and two suggest that in early adolescence, when people are just starting to drink alcohol, alcohol use is of minor predictive value for drinking patterns later on. Another test of the predictability of drinking over time, with evident implications for practice, are the analyses in which more problematic drinking behavior in late adolescence was predicted. These analyses showed that frequency of $6+$ drinking and frequency of drunkenness could hardly be predicted by drinking variables three years earlier (except the frequency of $6+$ drinking of boys which could be explained quite satisfactory, $r^{2}=.23$ ). Additionally, for the regular smokers at wave two, it was difficult to predict their quantity and frequency of use at wave three (as was also indicated by the simple correlations).

The relatively low stability of smoking and drinking within the category of users might be explained by measurement errors. Although the frequency and quantity measures were constructed out of two or more variables, there still might be substantial errors in measurement. In particular when substances are infrequently used, people might have problems remembering their use in the last month. On the orher hand, when a person drinks in a pub every weekend, the specific setting and actuality might result in a more accurate recall of alcohol (e.g., or cigarette) use (chapter 11; Sobell, Sobell, Leo \& Cancilla, 1988). A second limitation in this study is related to the low prevalence of smokers and drinkers in early adolescence. The small number of subjects in the analyses on the stability of amount of smoking and 
drinking leads to an increased susceptibility for the effects of outliers. Therefore, these findings should be interpreted with caution. Further research concentrating on late childhood and early adolescence should include large numbers of respondents to study stability of substance use over time.

\section{Transitions through States}

The correlations between the states over time suggested a relatively high stability of smoking and drinking. Nonetheless, when these relationships are examined in more detail as is done by computing transition matrices, it is evident that this stability is due to high stability in certain groups, namely the non-users and the regular users. Among the occasional users, there is strong instability with some subjects returning to a non-using status and other subjects shiffing to more frequent use. Of course, this is to some extent to be expected from the perspective of a stage approach. The likelihood of being a regular smoker at wave two is linearly related to allocation in the states at wave one. Thus, the probabilities as depicted in the columns provide evidence for a stage approach (see also Grant et al., 1987; Hirschman et al., 1984; Murray et al., 1983).

This is, however, only one interpretation of the transition matrices. The picture described above alters if one studies the rows in the matrices. The incidence of, for instance, daily smoking at wave three, is to a large extent due to the experimental smokers $(n=92)$ at wave two instead of the occasional smokers at wave two $(n=65)$. So reading the probabilities only, might lead to incorrect conclusions. This might be bad news for the development of prevention programs. Should one focus on the non-smokers, experimental smokers or occasional smokers in middle adolescence in order to prevent daily smoking later on? It is assumed that the interval between the waves (two and three years, respectively) might be too long to comprehend the dynamic nature of development of smoking and drinking. The lengthy follow-ups invite mis-classification bias with respect to smoking and drinking status and, subsequently, errors in the description of trends over time (see Grant et al., 1987, p. 254). Perhaps if shorter intervals were used between the waves, more support would be found for the stage approach. We stress that research is required to gain insight into a stage approach because recent studies demonstrated that it is likely that different processes and mechanisms are related to transitions through stages (e.g., Chassin, Presson, Sherman \& Edwards, 1990; De Vries \& Backbier, 1994; DiClemente et al., 1991). If, indeed, predictors of the transition from non-smoking to experimental smoking are different from the transition from occasional smoking to daily smoking, research on how (and at what pace) people move through states is warranted. 
In early adolescence, youngsters drink under parental supervision and smoke with peers. Throughout the years, they start to drink at parties, special evenings and public drinking places. Youngsters' smoking habits develop differently: they start to smoke in peer settings and then, as time goes on, they start to smoke at home. These findings coincide with studies of Wilks and Callan (1988), Presti et al. (1992) and Friedman et al. (1985), and stress the relevance of separating the different routes of smoking and drinking initiation.

That more youngsters smoke at home through the years might be explained by (a) the increasing age of the respondents or/and (b) the duration of use. At least, our data clearly show that continuing smokers more often smoke at home and in their own room compared to new smokers. The results are less clear for drinking. Although some differences between starters and continuing drinkers were displayed in mid-adolescence, no differences were found in early and late adolescence.

A limitation of our data is that the bipolar scale we used to register the frequency of smoking and drinking (i.e. at least once in the past four weeks) might not be precise enough. Particularly in the case of substance use in late adolescence, with a high prevalence of drinking and smoking in peer settings, this scale may not provide enough variation. Data on frequency and quantity of drinking and smoking in various settings will lead to more insight in the uptake of these substances in adolescence.

\section{NOTES}

${ }^{1}$ A shorter version of this chapter (with a focus on changes in substance use on the individual level) is submitted for publication as:

Engels, R.C.M.E., Knibbe, R.A., \& Drop, M.J. (1998). Changes in smoking and drinking behavior in adolescence: A five-year three-wave longitudinal study.

${ }^{2}$ Many researchers do not provide accurate data on response and attrition rates in their reports. An unpublished review of 43 papers concerning longitudinal surveys among children or adolescents showed that in 13 studies $(30 \%)$ no information was provided about attrition rates and that in 4 studies (9\%) incomplete or rather puzzling information was provided.

${ }^{3}$ Since the questions on the levels of alcohol consumption were not included in the telephone interview at the third wave, corresponding analyses were restricted to those who filled out a questionnaire at the second wave (90\% of the sample).

4 Although the Trimbos institute also conducted a study in 1988 (Plomp, Kuipers \& Van Oers, 1989), which is closer to our first wave (i.e., 1989), differences in operationalizations of variables would probably lead to inpossible comparisons. 



\section{HOMOGENEITY OF SMOKING BEHAVIOR IN PEER GROUPS: INFLUENCE OR SELECTION? 1}

Merlijn (boy, 12 years old): "Then you will join them. If you do not, you are not part of the group. You are afraid that they will judge you as stupid." Tamara (girl, 12 years old): "You do not have to lie about it. I just say: I do not want to. If they think you are stupid, it is still their problem." (reactions at the program 'Lifestyle for youth'; STIVA-news, 1995).

Peer groups are assumed to be of major importance for the development of cigarette smoking, alcohol use and drug use (Oetting \& Beauvais, 1992; Petraitis, Flay \& Miller, 1995). Throughout adolescence, youngsters experience feelings of uncertainty about their self-image, and consider themselves more or less dependent on the opinions and judgments of peers. Meeting the expectations of one's group is essential for preventing loss of friends, becoming a loner, and eventually losing one's (social) identity (Gordon, 1986). Therefore, associating with friends will be less complicated when own preferences and those of others are congruent. Studies carried out in the 1970 s focusing on determinants of drug use among adolescents, underline the assumption that there is a strong tendency towards similarities in behaviors and preferences with respect to adolescent friendships (Cohen, 1977; Jessor \& Jessor, 1977; Kandel, 1978).

Homogeneity of health related behavior in peer groups is caused by two processes: influence and selection. Influence refers to the assumption that an individual group member's behavior or opinions are affected by other members. Selection can be divided into two conceptually different mechanisms. Adolescents acquire new friends with similar characteristics, attitudes and behaviors. On the other hand, adolescents can turn down contacts with potential new friends, or even break off friendships because of differences in opinions and behaviors. 'This study' will examine to what extent homogeneity of cigarette smoking within peer groups can be attributed to either influence or selection processes. 


\section{Influence and Selection}

Recent refinements of theoretical models explaining substance use by adolescents have tried to unravel the concept of social influences (Petraitis et al., 1995). According to Graham, Marks and Hansen (1991) different types of influences must be distinguished. Through their perception of group members' behavior, young people receive information which can be interpreted normatively. Furthermore, they observe the positive and negative physical or social outcomes of the behavior of others. In order to comply with implicit requirements of members, to gain support and affection, or to stay in the group, one might start smoking.

Besides the modeling effects of perceived behavior by friends, pressures and explicit offers by peers are expected to contribute to the initiation of smoking. A longitudinal study by De Vries, Backbier, Kok and Dijkstra (1995) shows that smoking by significant others does play a significant role as a determinant of cigarette smoking, even when other environmental factors such as norms, pressures and offers and cognitive factors such as attitudes, self-efficacy and intentions are entered into the equation. Further studies extrapolating the Fishbein and Ajzen framework (Fishbein \& Ajzen, 1975) underscore the importance of smoking by peers as a robust predictor of smoking onset (Chassin, Presson, Sherman, Corty \& Olshavsky, 1984; Flay et al., 1994). Prevention programs have adopted the general idea of peer influences by arranging sessions in which young people are trained to cope with peer pressures (Botvin, Eng \& Williams, 1980; Bruvold, 1993; Evans et al., 1978).

Theoretical and intervention studies tend to interpret homogeneity in smoking in friendships as due to peer influence. A prominent reason for this assumption is due to the cross-sectional design of most research: sellection processes can be detected exclusively in prospective research. Only when information about the stability of friendships and behavior is obtained, can conclusions about the causes of homogeneity be drawn. Longitudinall research on similarities in cigarette smoking (Ennett \& Bauman, 1994; Fisher \& Bauman, 1988), drug use (Kandel, 1978), sexual behavior (Billy \& Udry, 1985), alcohol consumption (Fisher \& Bauman, 1988), and attitudes to delinquency (Jussim \& Osgood, 1989) explicitly show that selective association provides a comparable, or even better interpretation of homogeneity in friendships than influence processes. The authors of these studies emphasize that peer influences are overvalued as a predictor of individual behavior. An approach which includes selection processes sheds a new light on the role of adolescents in interpersonal relationships. It might be that young people are to a lesser extent passive actors, who are left to the mercy of environmental influences, and to a greater extent actively choose their own friends on the basis of similarities in attitudes, preferences or behavior. 


\section{Study Purposes}

The aim of this study was to examine whether influence and selection processes determine homogeneity of cigarette smoking in peer groups. Data for analyses were derived from a three-wave longitudinal study among secondary schoolchildren in the Netherlands (Van Reek, Knibbe \&c Van Iwaarden, 1993). Our approach will contribute to other social network studies in three ways. Firstly, this paper will not concentrate on friendship pairs but focus on the proportion of smokers within a peer group. In this way mechanisms can be analyzed at a group-level instead of a dyadic-level. Furthermore, some studies report difficulties with the required changes in configuration of the peer group, and behavioral patterns such as cigarette smoking by adolescents, at the follow-up survey. By employing a study with intervals of two and three years between the waves, we have tried to accommodate these complications.

\section{METHOD}

\section{Procedure and Subjects}

In 1989 the study started with the selection of 65 secondary schools differentiated by educational level and region. Formal consent was obtained from 36 schoolboards. Refusals to participate concerned privacy considerations, or affiliation with orher research or intervention programs. Questionnaires were administered to students of first and second grade, and filled out in the classroom. ${ }^{2} 1,454$ subjects were enrolled in the baseline measurement. Special attention was paid to the confidentiality of responses. The letters of introduction and the questionnaires emphasized privacy aspects, and clearly stated that no information about specific responses of subjects would be passed on to teachers or parents. Furthermore, matching of numbers and names was only possible by the principal researcher.

At the follow-ups, two and five years after the first measurement, forms were send to youngsters" homes. More than $76 \%$ of the adolescents returned questionnaires at the second wave, and $72 \%$ at the third wave. In 1991 parents of subjects who did not respond were contacted for permission to complete an interview with their son or daughter by phone. In 1994 subjects who did not return the questionnaire were directly approached for a telephone-interview. The response rates were $82 \%$ at both follow-up surveys. A total of 1,063 adolescents $(73 \%)$ were enrolled in all three waves.

Attrition analyses are reported elsewhere (see chapter 4). A logistic regression analysis showed no significant differences $(p>05)$ in frequency and intensity of tobacco use between remainders (e.g. participation at all three waves) and drop outs (participation at one or two waves). 
The sample consisted of $520(49 \%)$ girls and $543(51 \%)$ boys who supplied data at all waves. The average age of subjects was 12.4 years $(s d=.69)$ at wave one, 14.4 at wave two and 17.4 at wave three. In $1989,36 \%$ of the subjects were enrolled in vocational schools, and $64 \%$ went to high schools. Twenty-six percent of the subjects reported that their farher was Catholic, 27\% Protestant, 4\% Islamic, 5\% had a different religion and $38 \%$ had no religion. Sixteen percent attended a church weekly, $32 \%$ less often and $52 \%$ never attended a church. The majority of the adolescents (85\%) lived with two parents (and no stepparent). Eighty-nine percent of the adolescents had a father of Dutch origin.

\section{Measures}

Smoking status was assessed by asking subjects to indicate their frequency of smoking during the past four weeks. Responses were recoded to a dichotomous variable differentiating smokers (smoked in the past four weeks) and non-smokers (did not smoke in the past four weeks). This variable was measured at all three waves.

Peer smoking was measured by asking about the proportion of smoking friends in their immediate peer cluster on a 5-point scale with anchors 1 'no one', 2 'less than half of the peer group', 3 'half of the peer group', 4 'more than half of the peer group' and 5 'all members of the peer group smoke'. Responses were recoded to a dichotomous variable differentiating predominantly non-smoking (score 1,2 or 3) and predominantly smoking (score 4 or 5). This item was included in all three waves.

Changes in the formation of the subjects' peer group were assessed at the third wave (1994) by asking youngsters whether their current peer group configuration corresponded to the peer group of the second wave. Response categories were positioned on a 5-point scale ranging from 1 'no changes in the composition of my peer group', 3 'half of the peer group has changed' to 5 'I have a completely new peer group'. Since only $7 \%$ of the respondents reported to have no changes in their peer group, those with a score of 1 or 2 were labeled as limited changes and with a score 3 or more as substantial changes.

\section{Data Analyses}

Owing to information about changes in the peer group being obtained at the last wave, the analyses concerned changes between the last two follow-ups. At first, we examined to what extent peer groups are homogenous in smoking behavior. Crosssectional associations between adolescent and peer smoking were analyzed for the second (T2) and third wave (T3) using contingency tables with chi-square $\left(x^{2}\right)$ statistics. 
In order to test influence processes analyses were restricted to the selection of subjects who reported none or only small changes in the composition of their peer group. This restriction was required to ensure that selection processes did not interfere. Influence processes were examined by looking at the impact of peer smoking at T2 on subject" smoking at T3. Separate contingency tables were analyzed for adolescent smokers and non-smokers at T2.

Selective association was examined for those subjects who reported substantial changes in their friendships, or who had a totally new peer group. Selective association in terms of the acquisition of new friends with similar smoking habits was analyzed by examining whether the smoking status of adolescents at T2 appeared to be a predictor of the smoking status of their 'new' peer group at T3. Distinction is made between subjects with a stable smoking status, where possible peer influence is minimized, and the total group. This latter analysis is added to enhance the generalization of our findings.

Deselection, dropping of friends with dissimilar smoking status, was examined by considering whether differences in smoking status at T2 turned out to be a reason for changes in peer group configuration at T3. It was expected that if subjects experience differences in smoking status they will be more inclined to change their peer group than those who experience no differerences. For these analyses only those subjects with stable smoking status were included. Otherwise it was possible that influence processes interfered. The aforementioned analyses are presented as contingency tables with chi-square statistics.

\section{RESULTS}

\section{Changes in Peer Group and Smoking Behavior}

Variability and stability of friendships must be reported in order to investigate influence and selection processes. Forty-five percent of the sample indicated that there were merely minor changes in their peer group in the last three years. According to $55 \%$ of the subjects, half (or more) of their peer group consisted of new friends.

Of the 1,063 subjects involved, 215 adolescents (20\%) were classified as smokers at the second wave and 439 adolescents ( $41 \%$ ) at the third wave. At wave two, $63 \%$ indicated being affiliated with a predominantly non-smoking peer group, while $23 \%$ reported being associated with a predominantly smoking peer group. Fourteen percent indicated not being aware of the proportion of smokers within their peer group or did not answer this particular question. In $1994,56 \%$ had a nonsmoking peer group, $40 \%$ a smoking peer group and $4 \%$ did not provide information. If no data of the peer group smoking status was retrieved, subjects were excluded from analyses. 
Table 3.1. Cross-sectional Associations between Adolescent and Peer Group Smoking Status (in row percentages, and sample size in parentheses)

\begin{tabular}{|c|c|c|c|c|}
\hline \multirow{3}{*}{$\begin{array}{l}\text { Peer group } \\
\text { smoking status }\end{array}$} & \multicolumn{4}{|c|}{ Adolescent smoking status } \\
\hline & \multicolumn{2}{|c|}{ Wave 2} & \multicolumn{2}{|c|}{ Wave 3} \\
\hline & Non-smoker & Smoker & Non-smoker & Smoker \\
\hline $\begin{array}{l}\text { Predominantly } \\
\text { non-smoking }\end{array}$ & $\begin{array}{c}89 \\
(603)\end{array}$ & $\begin{array}{c}11 \\
(72)\end{array}$ & $\begin{array}{c}76 \\
(442)\end{array}$ & $\begin{array}{c}24 \\
(142)\end{array}$ \\
\hline $\begin{array}{l}\text { Predominantly } \\
\text { smoking }\end{array}$ & $\begin{array}{c}47 \\
(112) \\
\end{array}$ & $\begin{array}{c}53 \\
(129) \\
\end{array}$ & $\begin{array}{c}33 \\
(139) \\
\end{array}$ & $\begin{array}{c}67 \\
(281)\end{array}$ \\
\hline
\end{tabular}

Note. Figures are presented in percentages. Statistics for wave two contingency tables; $\chi^{2}(1, n=$ $916)=190.47, p<.001$. Statistics for wave three contingency tables; $\chi^{2}(1, n=1,004)=$ $181.76, p<.001$.

\section{Cross-sectional Associations of Smoking by Adolescents and Friends}

If a subject had a predominantly non-smoking peer group, the probability of being a non-smoker emerged as being $89 \%$ (T2), and $76 \%$ (T3) (Table 3.1). The probabilities of being a smoker, among those with a predominantly smoking peer group, were $53 \%$ (T2), and $67 \%$ (T3). Correlates between subjects' use and peer use measured on the original 5-point scale supported these findings. Positive associations were found at $\mathrm{T} 2(r=.52, p<.001)$, and $\mathrm{T} 3(r=.48, p<.001)$.

Table 3.2. Influence Processes: The Impact of Peer Smoking on the Onset of Adolescent Smoking for Non-smokers at T2 with a Stable Peer Group (in row percentages, and sample size in parentheses)

\begin{tabular}{lcc}
\hline Peer group & \multicolumn{2}{c}{ Adolescent smoking T3 } \\
\cline { 2 - 3 } smoking T2 & Non-smoker & Smoker \\
\hline Predominanty & 75 & 25 \\
non-smoking & $(200)$ & $(66)$ \\
& & \\
Predominantly & 65 & 35 \\
smoking & $(28)$ & $(15)$ \\
\hline
\end{tabular}

Nore. For subjects with a stable peer group; $\chi^{2}(1, n=309)=1.94, p=.16$.

\section{Influence Processes}

Of the adolescents associated with a predominantly non-smoking peer group at $\mathrm{T} 2$, $25 \%$ took up regular smoking habits three years larer (Table 3.2). Of adolescents with smoking friends at $\mathrm{T} 2,35 \%$ reported smoking at $\mathrm{T} 3$. These differences did not 
reach significance $(p=.16)$. In an analysis with all subjects, stable and unstable peer groups, $27 \%$ of adolescents with a predominantly non-smoking peer group became smokers three years later, while $40 \%$ of subjects who had smoking peers began smoking. Results were significant with $\left.\chi^{2}(1, n=715)=8.36, p<.01\right)$. Smoking cessation was relatively rare in this study. A few subjects $(n=24)$ appeared to quit their regular smoking habits three years later. Within the subsample of adolescents with stable friendships only five people quit smoking. It was, therefore, not possible to examine the effects of peers on smoking cessation.

\section{Selection Processes}

Since selective association exclusively concerned adolescents with new friends at $T 3$, we investigated whether the subjects smoking status at $T 2$ was related to the extent of changes in friendships. This was not the case in the present study. Fifty-four percent of the actual smokers, and $55 \%$ of the non-smokers reported acquiring new friends in their peer group $\left(\chi^{2}(1, n=1,041)=.05, p>.10\right)$.

Adolescents tended to associate with those peers who corresponded in smoking status (Table 3.3). Non-smoking subjects established new relationships with persons with similar smoking status $(63 \%)$, rather than with dissimilar smoking status $(27 \%)$. Smoking youngsters were more likely to associate with other smokers (63\%). This pattern was more distinct for adolescents who remained stable in their smoking behavior between the waves. Only $25 \%$ of the T2-non-smokers was affiliated with a predominantly smoking peer group at T3, while $70 \%$ of the T2smokers was associated with smoking friends.

An additional analysis was conducted for those who reported having a completely new peer group $(n=94)$. The possibility of interference of influence processes is reduced because none of the friends at T3 were also peer group members at $T 2$. The findings are quite similar to those described above. Sixty-five

Table 3.3. Selection Processes: The Impact of Adolescent Smoking at T2 on the Formation of Peer Groups at T3 for Those Who Reported Substantial Changes in their Peer Group (in row percentages, and sample size in parentheses)

\begin{tabular}{|c|c|c|c|c|}
\hline \multirow{3}{*}{$\begin{array}{l}\text { Adolescent } \\
\text { smoking T2 }\end{array}$} & \multicolumn{4}{|c|}{ Peer group smoking " $\mathrm{T}^{3} 3$} \\
\hline & \multicolumn{2}{|c|}{ Stable smoking status } & \multicolumn{2}{|c|}{ Total group } \\
\hline & Non-smoking & Smoking & Non-smoking & Smoking \\
\hline Non-smoker & $\begin{array}{c}75 \\
(225)\end{array}$ & $\begin{array}{c}25 \\
(76)\end{array}$ & $\begin{array}{c}63 \\
(275)\end{array}$ & $\begin{array}{c}37 \\
(165)\end{array}$ \\
\hline Smoker & $\begin{array}{c}30 \\
(28)\end{array}$ & $\begin{array}{r}70 \\
(65)\end{array}$ & $\begin{array}{c}37 \\
(41)\end{array}$ & $\begin{array}{l}63 \\
(70)\end{array}$ \\
\hline
\end{tabular}

Note. For subjects who were stable in smoking status; $\chi^{2}(1, n=394)-61.62, p<.001$. For the total subsample; $\chi^{2}(1, n=551)=23.68, p<.001$. 
percent of the smokers was associated with a predominantly smoking peer group at T3 while $61 \%$ of the non-smokers was associated with non-smoking friends $\left(\chi^{2}(1, n\right.$ $=90)=4.82, p<.03$ ).

No data was retrieved about the smoking status of individual friends within the peer group of subjects. Neither could the continuation of friendships be investigated, nor the dropping out of friends from the youngsters' peer group. Therefore, deselection of friends in order to achieve similarity in smoking status, was analyzed on an aggregate level. Outcomes depicted in Table 3.4 do not provide evidence for the existence of deselection processes. Cross tabulations for smoking and non-smoking adolescents were nonsignificant $(p>.10)$.

Table 3.4. Deselection Processes: The Impact of Differences in Smoking Status between Adolescents and their Peer Group at T2 on the Deselection of Friends at T3 for Those Who Were Stable in Smoking Status (in row percentages, and sample size in parentheses)

\begin{tabular}{lccccc}
\hline & \multicolumn{4}{c}{ Changes in peer group formation } \\
Peer group & \multicolumn{1}{c}{ Non-smoking adolescent } & & Smoking adolescent \\
\cline { 2 - 3 } \cline { 5 - 6 } smoking T2 & Limited & Substantial & & Limited & Substantial \\
\hline Predominantly & 46 & 54 & & 45 & 55 \\
non-smoking & $(200)$ & $(236)$ & & $(27)$ & $(33)$ \\
& & & & 50 & \\
Predominantly & 43 & 57 & & 50 \\
smoking & $(28)$ & $(37)$ & & $(56)$ & $(57)$ \\
\hline
\end{tabular}

Note. Contingency tables were not significant at the $p<.10$ level.

\section{DISCUSSION}

The present study examines to what extent homogeneity of smoking in peer groups can be explained by influence and selection processes. Before this question could be answered, we tested whether peer groups are indeed homogenous in smoking. Cross-sectional analyses showed that respondents and their peers are quite similar in smoking status.

Findings with regard to peer influences are not straightforward. For the total sample, the probability of becoming a smoker appeared to be higher for adolescents who were associated with a predominantly smoking peer group (40\%) than for adolescents with a predominantly non-smoking peer group $(27 \%)$. However, when the analysis was restricted to stable peer groups, the effects of peers on individual behavior did not reach significance.

Selection processes can only be detected if people report transformations in their social relationships. Our results show that most of the adolescents' friendships are open for change - only a small number of respondents indicated having an unchanged peer group over a period of three years. Strong support was found for 
selective association. In general, adolescents choose those peers with corresponding smoking status. Young people with stable smoking status were most systematic in their choice of friendships: smokers were more likely $(70 \%)$ to affiliate with other smoking peers. Furthermore, only one out of four non-smoking adolescents indicated that their peer group was predominantly smoking.

No evidence was found for deselection processes. It is possible that differences in smoking are not important enough to break off existing friendships. However, further research should examine which factors are accountable for discontinuity of friendships, and explore the role of smoking, or other substances, in these considerations.

Our findings which are mainly based on analyses at an aggregate (group) level are, in general, consistent with previous network studies (Billy \& Udry, 1985; Cohen, 1977; Ennett \& Bauman, 1994; Jussim \& Osgood, 1989; Kandel, 1978). Most of these studies found only little or no evidence for the effects of friends' behavior causing homogeneity of behavioral patterns in peer groups. Moreover our study support the outcomes of a recent review (Bauman \& Ennett, 1996) which stress the relevance of selective association. The fact that discrepancies in smoking status within peer groups were not associated with discontinuity of existing relationships is supported by other studies (Ennett \& Bauman, 1994; Fisher \& Bauman, 1988). Consequently, we underscore that similarities of smoking within friendships found in cross-sectional studies should more often be regarded as the result of selective association.

The main analyses presented in this paper were conducted with data, derived from two measurements with an interval of three years. Therefore, it is hardly possible to comprehend the dynamic nature of friendships. Which transitions in behavior or friendships occurred in the intervening years could not be explored. It would be worthwhile to scrutinize whether selection or whether adaption processes could take place in short periods of time, or certain sequences in processes can be identified. Certain factors might effect the magnitude of different processes. For instance, youngsters with low self-confidence could be more dependent on the continuation of contacts, and may be more susceptible to potential peer influences. On the other hand, adolescents with high self-confidence might be more autonomous and be more competent in resisting influences, or choosing their own friends.

\section{Limitations of the Present Study}

The progression from experimentation to daily smoking is considered to be based on distinct stages (Leventhal \& Cleary, 1980). Some studies are based on the idea that stages are linked to different behavioral patterns while others describe cognitive as well as behavioral considerations (Werch \& Anzalone, 1995). Dividing subjects into categories of regular smokers and non-smokers suggest that smoking is bipolar 
and not associated with stages, whilst in fact youngsters who are experimenting with cigarettes, or are even developing positive attitudes towards smoking may be vulnerable to the effects of (non)smoking peers. It would be of interest to explore selection and adaption processes from the perspective of homogeneity in attitudes and intentions as well as behavioral aspects.

The definition of a peer group differs across studies. Sometimes does it refer to a specific age category, the students at school, kids in the neighbourhood or to the inner circle of friends. The latter meaning, which provides the most information regarding influence and selection processes was used in this study. Furthermore, besides the best friend or the peer cluster, it would be worthwhile to pay attention to the impact of dating partners on smoking habits.

Respondents supplied detailed information about the smoking status of referent others. These kind of self-reports have the drawback that smokers tend to exaggerate the prevalence of smoking in society, and in their peer group in particular (Marks \& Miller, 1987; Sherman, Presson, Chassin, Corty \& Olshavsky, 1983). Another potential drawback deals with the use of self-report measures of actual smoking. In this study, a biochemical test of smoking was not entailed. Because administration of the questionnaires took place at subjects' homes at the two follow-ups, it was from a logistic (and financial) point of view hardly possible to conduct these tests. In addition, it is unknown if people can remember the specific changes in their peer group(s) over a period of three years. Further research must reveal whether errors in estimations of peer smoking or changes in peer groups, or in their own smoking history, are related to the strength of the mechanisms discussed in this report.

The fact that no extensive support was found for peer influences might be due to the assessment of this concept. Although the effects of peer smoking is an often employed indicator of peer influences, perceived pressures or explicit offers could be prominent in affecting adolescent use (Evans et al., 1978; Graham et al., 1991). However, if there is no or little evidence for modeling effects, one might doubt the role of more explicit influences of smoking others.

\section{Implications for Practitioners}

How can the acquired knowledge be implemented in health promoting programs? First, we must state that this paper deals with the interpretation of homogeneity of regular smoking within groups, and does not explicitly focus on factors affecting the initiation of smoking. The most prominent finding of this study is the relative contribution of peer influences as a determinant of homogeneity in smoking behavior within peer groups. Programs which are based on an 'influence' approach must acknowledge the impact of processes of selection.

This study shows that adolescents report substantial transformations in their peer group. Although discrepancies in smoking behavior did not cause friendships 
to break off, the selection of new friends was influenced by whether there was similarity or not in smoking status. Creating awareness of these more or less implicit choices might contribute to more effective programs. In our view, health educators should focus on future friendships along with the present peer group.

Many smoking prevention programs for adolescents concentrate on teaching social skills and strengthening self-efficacy (Botvin et al., 1980; Bruvold, 1993; Evans et al., 1978). The fact that peer influences might be somewhat overvalued as predictors of regular smoking advocates concentration on other determinants. Especially, premature positive attitudes towards tobacco of young children and norms exhibited by parents might affect influence and selection processes at a later age. In addition, the limited effects of peer influences emphasize a renewed attention in research to other sources of social influence such as parents, siblings, other adults, teachers, media (e.g. advertisements, television).

Finally, quitting rates were remarkably low among adolescents who were regular smokers. Although it is feasible that in earlier stages of smoking, decisions are made about maintenance or cessation of smoking, interventions are still needed to enhance adolescents' efforts, intentions, and strategies to quit smoking.

\section{NOTES}

"Based on:

Engels, R.C.M.E., Knibbe, R.A., Drop, M.J., \& De Haan, J.T. (1997). Homogeneity of smoking behavior in peer groups: Influence or selection? Health Education and Bebarior, 24 , 801-811.

Engels, R.C.M.E., Knibbe, R.A., Drop, M.J., \& De Haan, J.T. (1996). Invloed en selectie in vriendschapsrelaties van adolescenten: Een longitudinale studie naar homogeniteit in rookgedrag. Gedrag en Gezondheid, 23, 257-268.

${ }^{2}$ In the Netherlands, people follow primary education until they are about 12 years old. Then they follow secondary education for 4 to 6 years (depends on level of education). The baseline measurement included youngsters who were in first and second grade and in the age category 12 to 14 years. 



\section{INFLUENCES OF PARENTAL AND BEST FRIENDS' SMOKING AND DRINKING ON ADOLESCENT USE ${ }^{1}$}

Many researchers acknowledge the importance of social influences with respect to development of adolescent substance use. However, it is still debatable whether it is parents or peers who most strongly affect adolescents' behavior. In this article, we contribute to this discussion by proposing a model which examines differential influences of perceived behavior of friends and parents on youngsters' smoking and drinking behavior. Studies have rarely explored the relative influences of both friends and parents simultaneously over an extended period of time. The present study addresses these limitations by employing a longitudinal design in which a sample of adolescents was followed during their secondary school years.

\section{Impact of Parents and Peers}

Associations between substance use by parents and their offspring have been attributed to several mechanisms, such as the impact that parents have as models (Akers, Krohn, Lanza-Kaduce \& Radosevich, 1979; Bandura, 1977), and the increased availability of cigarettes and alcoholic beverages when parents are users (e.g., Kandel, 1978; Skinner, Massey, Krohn \& Lauer, 1985; Stein, Newcomb \& Bentler, 1987). Others suggest that especially youngsters with strong family bonds are likely to imitate behavior (Krosnick \& Judd, 1982). Biddle, Bank and Marlin (1980) hypothesized that children are affected by parental use through internalization of family norms, values and life-style. Although different explanations are provided for similarities of behavior in parent-child relationships, the literature is generally affirmative about the fostering effects of parents at 
initiation and maintenance of adolescent substance use.

Cross-sectional studies show that youngsters who smoke or drink are more likely to be exposed to a 'using' family background (e.g., Biglan, Severson, Bavry $8 x$ McConnell, 1983; Chassin et al., 1981; Dusenbury, Epstein, Botvin \& Diaz, 1994; Jensen \& Overgaard, 1993; Nolte, Smith \& O'Rourke, 1983). In a review of findings of prospective studies on predictors of onset of smoking, Conrad, Flay and Hill (1992) reported, however, that in six out of eleven studies parental cigarette use appeared not to be associated with children' use at a later age. In some studies parental use was only a determinant for girls and not for boys (Charlton \& Blair, 1989; Chassin, Presson, Sherman, Montello \& McGrew, 1986). Only a few longitudinal studies have been conducted to examine effects of parental alcohol use. A recent study by Green, Macintyre, West and Ecob (1991) found that the drinking behavior of both parents was associated with alcohol use by their offspring after a 12 to 18 month follow-up, but only for families with a non-manual occupational background.

In many sociall psychological and sociological models, peer group influence is considered to be a major cause of initiation of smoking or drinking, as it is assumed that youngsters are vulnerable in their attempts to join and remain in certain peer groups (see for theoretical review Petraitis, Flay \& Miller, 1995). It has been postulated that when experiencing dissimilarities between their own and a friend's behavior, preferences or attitudes, adolescents try to resolve discrepancies by adopting friends' smoking or drinking habits (e.g., Oetting \& Beauvais, 1986). Smoking prevention programs are, therefore, designed to teach young people how to resist pressures to smoke (Flay, d'Avernas, Best, Kersell \& Ryan, 1983; Telch, Miller, Killen, Cooke \& Maccoby, 1990). At secondary schools, video-material and role plays are incorporated into programs to generate awareness of the influences of peers on substance use (Eiser, Morgan, Gammage, Brook \& Kirby, 1991).

Numerous cross-sectional studies have demonstrated the strong positive relationship between adolescents' alcohol use or smoking and that of their friends. Evidence was provided for similarities of smoking and drinking status in friendships by using data from adolescents and corresponding friends (Biddle et al., 1980; Wilks, Callan \& Austin, 1989). Other studies emphasize the effect of friends' smoking on the intention to smoke (Chassin et al., 1981; Evans, Dratt, Raines \& Rosenberg, 1988). Longitudinal studies of smoking (Flay et al., 1994; Ferguson, Lynskey \& Horwood, 1995), alcohol use (Johnson, 1988) and marijuana use (Aseltine, 1995) showed direct effects of friends" use on adolescents" use over time. In addition, according to the review of Conrad et al. (1992), friends' smoking predicted onset of smoking of the adolescent in 15 (out of 16) studies.

In general, the extent to which parental influences are apparent remains unresolved according to the discussed empirical research. However, it seems to be the case that peer influences are one of the most consistent and robust predictors of smoking and drinking behavior of young people. 


\section{Limitations of Designs and Aralyses}

Most longitudinal and cross-sectional research relies on the assumption that similarities in behavior among friendships must be attributed to peer influences. However, homogeneity of behavior in friendships can also be interpreted as the outcome of a process of choosing persons with equal characteristics (Bauman \& Ennett, 1996; Billy \& Udry, 1989; Cohen, 1977; Ennett \& Bauman, 1994; Hu, Flay, Hedeker, Siddiqui \& Day, 1995; Kandel, 1978; Urberg, Degirmenciogly \& Pilgram, 1997). In a prospective study, Fisher and Bauman (1988) found that similarities in cigarette smoking and alcohol use in friendship pairs were mostly due to selection processes and only rarely to peer influences. If selective attraction is considered to be a basis for the formation of friendships, this may change the dominant views on the impact of peer groups. In addition, Aseltine (1995: 116) concludes that '... these mutually-reinforcing processes highlight the need to emphasize social interaction, as opposed to social influence, in general theories of problem behavior'. Until recently, however, interactions in friendships were often neglected in this field of research. Most study designs do not permit separation of bilateral influences. Eventually this might lead to an overestimation of the amount of peer influences (Urberg, Cheng \& Shyu, 1991).

Another problem in this field of research concerns the role of past behavior. Many studies reported that earlier experimentation with substance use appears to be a strong determinant of use later on (e.g., Pederson \& Lefcoe, 1987; Farell, 1994; Ferguson et al., 1985; Graham, Marks \& Hansen, 1991; Hu et al., 1995; Stein et al., 1988). As Graham and coworkers (1991) point out, omission of previous substance use by adolescents from analyses could lead to an overestimation of the effects of other predictors. For example, one may found spurious peer effects due to the crosssectional correlation between prior behavior and prior peer use. A few strategies are apparent to examine selection effects. First, one could use different subsamples existing of stable and unstable peer clusters. When stable friendships are examined, effects of friends" substance use on participants' use could be interpreted as due to influence because the chances that selection effect interfere are limited. Using unstable friendships, the extent to which homogeneity in peer smoking can be ascribed to selective association by the participant can be investigated (Urberg et al., 1997). Second, selection effects could be investigated by using cross-lagged paths between friends use and participants use in an analytical model. This implies that previous behavior of both peers and participants are included in analyses. The present study examines the effects of peer and parental smoking and drinking in the context of effects of previous use by adolescents themselves.

The presence of smoking or drinking parents may interact with the potential effects of use by friends. For instance, when young people experience the impact of smoking parents, the perceptions of smoking friends could be redundant in influencing subjects own behavior: they are already affected by parental use. On the 
contrary, for youngsters who are not exposed to smoking parents, friends' smoking might affect behavioral patterns. This hypothesis will be tested in our study.

\section{The Present Study}

This study addresses three major issues. In the first place, we explore whether cigarette smoking and alcohol use by parents and best friends foster adolescents' use over time. To optimize the possibility of friends influencing the adolescents' use, this question will be addressed both for the total sample and for the selection of adolescents who grow up within a non-using family. Secondly, attention is paid to the impact of adolescents themselves on similarities of substance use in friendships. Thirdly, this study will also focus on the influence of previous substance use, which will allow a more realistic estimate of the predictive value of the influence of parents and friends.

A model was constructed to represent the relative influences of referent others on smoking and drinking of youngsters (Figure 4.1). The postulated causal model was tested with self-report data gathered at three time points, with intervals of two and three years, respectively. Although constructs may be transversely

Time 1

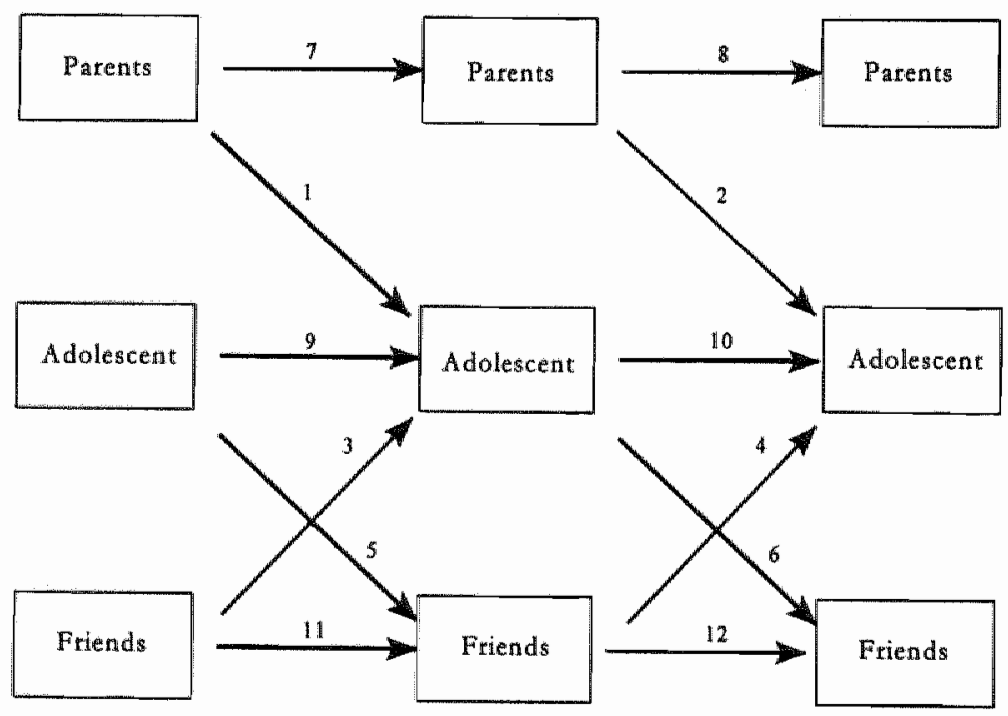

Figure 4.1. Theoretical Model of Relationships between Best Friends', Parental, and Adolescents' Smoking and Drinking Behavior. 
correlated, no assumptions were made about the directions of relationships on a cross-sectional level. Numbers were assigned to different paths to illustrate our hypotheses.

Parental smoking and drinking is hypothesized to directly foster adolescent use over time (paths 1 and 2). Moreover, similarities in the behavior of parent and child are assumed to be exclusively due to the child adopting the parent's behavior: it is not very likely that adolescents are responsible for changes in parental use. Cross-lagged effects between adolescents' and best friends' behavior are hypothesized as follows: exposure to smoking or drinking by best friends is of influence on adolescents' own substance use $(3,4)$. However, as argued before, adolescents' own substance use may affect the selection of friends. Therefore, the model will test to which extent adolescents' use is positively associated with subsequent best friends" use over time $(5,6)$. Direct effects of previous behavior by adolescents $(9,10)$ on substance use over time are expected. Finally, it is hypothesized that cigarette smoking and alcohol use by parents $(7,8)$ and best friends $(11,12)$ are determined by levels of use at a preceding time point.

\section{METHOD}

\section{Procedure and Attrition Rates}

This study is part of a 5-year longitudinal research project concerned with predictors of smoking and drinking behavior of adolescents in the Netherlands (Van Reek, Knibbe \& Van Iwaarden, 1993). In 1989, the cohort study started with a selection of 65 schools differentiated to educational level and region. Formal consent from 36 school boards was obtained. Twenty-nine secondary schools refused to participate in the study. One reason was privacy; names and addresses of subjects were needed for follow-up surveys. Furthermore, participation in other research or intervention programs was a reason for refusal.

Special attention was placed on confidentiality of responses. Only the principal researcher could match the names and numbers. At the first measurement the questionnaires were administered in classrooms and returned by the subjects in a sealed envelope. 1,454 subjects of first and second grade of secondary education (mean age 12.4 years) completed the questionnaire.

In the second and third wave forms were sent to subjects' homes. In 1991, parents of the subjects who did not respond, were contacted for permission to interview their child by telephone. In 1994 (subjects' mean age 17,4), subjects were directly contacted to participate in the study. Eighty-two percent of the adolescents participated in the second wave, and $82 \%$ also was enrolled in the third wave. In total, $73 \%$ of the original sample participated in the two follow-up surveys. Response figures are depicted in Table 4.1. 
Table 4.1. Response Percentages

\begin{tabular}{llll} 
& Year & $\mathrm{N}$ & $\%$ \\
\hline $1^{\text {sf }}$ Wave & 1989 & 1,454 & \\
$2^{\text {nd }}$ Wave & 1991 & 1,192 & 82 \\
$3^{\text {rd Wave }}$ & 1994 & 1,187 & 82 \\
All three waves & & 1,063 & 73 \\
\hline
\end{tabular}

Note. 124 subjects were enrolled in the third wave, but not in the second wave.

A logistic regression analysis was conducted to compare participating subjects and the drop outs (i.e., not participating in wave two and/or three) with data of the first measurement as predictors of drop out. Independent variables were sex (1 male), age (ratio level), education ${ }^{2}$ (1 vocational school, 2 high school), church attendance (1 every week, 2 less often, 3 never), origin of the father (1 Dutch, 2 nonDutch), intensity of smoking (number of cigarettes smoked in the last week) and intensity of alcohol consumption (number of glasses of alcohol consumed in the last week). The indicators for frequency of smoking and drinking are described in the Measures section. The logistic regression analysis was conducted with the backward elimination method. The likelihood-ratio statistic was used to select variables for removal. The criteria used to control the selection of independent variables entering the model was $p<.10$, and for removal from the model $p<.15$ (Hosmer $8 x$ Lemeshow, 1989: 108).

Dropouts $(n=391)$ appeared to be older (Odd Ratio $(O R)=1.31,95 \%$ Confidence Interval $(C I)=1.12-1.54)$, lower educated $(\mathrm{OR}=.61,95 \% \mathrm{CI}=.47-$ $79)$, less often attended a church $(\mathrm{OR}=1.36,95 \% \mathrm{Cl}=1.13-1.64)$ and less often had a father of Dutch origin (OR $=3.20,95 \% \mathrm{CI}=2.35-4.36)$ compared to remainers $(n=1,063)$. Small differences were found for $\operatorname{sex}(\mathrm{OR}=.77,95 \% \mathrm{CI}=$ $.60-1.00, p=.053)$ and intensity of cigarette smoking $(\mathrm{OR}=1.01,95 \% \mathrm{CI}=1.00$ $1.02, p=.09$ ). Dropouts were more likely to be male and reported a higher number of cigarettes smoked than remainers. No differences $(p>15)$ were found for frequency of drinking and smoking, and intensity of drinking. Although there was small but detectable selective attrition, no salient differences between respondents and dropouts on relevant behavioral outcome variables were found.

\section{Subjects}

The sample consisted of 543 boys (51\%) and 520 girls (49\%). At the first wave the mean age was $12.4(S D=.69)$. In $1989,35 \%$ of the subjects were enrolled in vocational schools, and $65 \%$ went to high schools. Most youngsters (85\%) were in first grade of secondary education. Twenty-six percent of the students reported having a Cartholic father, $27 \%$ Protestant, $4 \%$ Islamic, $5 \%$ had a different religion and $38 \%$ had no religion. Sixteen percent of the sample attended a church weekly, 
$31 \%$ less frequently, and $53 \%$ never. Eighty-five percent lived with two parents (and no stepparents). Ninety-three percent of the adolescents had a father or mother of Dutch origin.

\section{Measures}

Parental smoking was assessed with the question: 'Do the following persons smoke?'. Subjects were asked to indicate smoking by father and mother on a 3-point scale (i.e. 'My father smokes/may smoke/does not smoke') (De Vries, Backbier, Kok \& Dijkstra, 1995). A sum index was constructed which ranged from 1 "neither of them smokes' to 5 'both smoke".

Parental alcohol use was measured by asking the respondents how often their father and mother consumed alcoholic beverages (responses ranged from 'daily', 'weekly', 'less than weekly' to 'never'). These scores were summed to one scale ranging from 1 'both parents are abstainers' to 7 "both parents drink daily".

Friends' smoking and drinking was assessed in comparable ways to parental substance use. Scales were composed for the two closest friends of the subjects. Friends' smoking was measured on a 5-point scale: 1 'both friends are non-smokers' to 5 'both are smokers'. The extent of alcohol use by friends was assessed on a 7 . point scale ( 1 'both friends are abstainers' to 7 'borh friends are daily drinkers').

Subjects' smoking was measured by the following questions. At all waves subjects were asked to indicate their life-time smoking: "Have you ever smoked a cigarette, even a few puffs?'. Furthermore, two questions were used to assess smoking in the last three months and the last four weeks. Response categories for smoking in the last four weeks were 'daily', 'weekly", 'less than weekly' and 'did not smoke in the last four weeks'. These questions were used to construct a smoking index on a 6-point scale; 1 'never smoked', 2 'had experimented with smoking, but did not smoke in the last 3 months', 3 'had experimented with smoking, but did not smoke in the last 4 weeks', 4 'smoked occasionally, less than weekly", 5 'smoked at least once per week', and 6 'smoked daily'.

Subjects' alcoholl use was assessed with an index constructed of three questions. For life-time drinking (separately for beer, distilled spirits and other beverages) subjects were asked if they ever drank alcohol, even a sip. In 1994, the past three years were used as a reference period. In addition, questions were asked about alcohol use in the last six months and in the last four weeks. Response categories for drinking in the last four weeks were 'daily', 'weekly", 'less than weekly' and 'did not consume alcohol in the last four weeks'. A classification of drinking behavior was constructed on a 5-point scale: 1 'total abstainer', 2 "experimental drinker, consumed alcohol in the last 6 months", 3 "experimental drinker, did not consume alcohol in the last 4 weeks', 4 'occasional drinker, consumed alcohol in the last four weeks' and 5 'regular drinker, used alcohol at least once a week'. 
Identical assessments of variables were used for all three waves. Subjects who did not provide information on two or more questions about parental and best friends' behavior, were omitted from analyses. Where subjects had one missing value on the twelve items concerning parental and best friends' smoking (also for drinking) it was substituted by the mean of that particular item. By this procedure, 113 subjects for cigarette smoking and 68 subjects for alcohol use were excluded from further analyses. Furthermore, when no information about adolescents' own substance use was retrieved, subjects were subsequently excluded from structural modeling analyses. For smoking behavior, 77 subjects did not provide data of their smoking on at least one of the three waves. Regarding alcohol use, 65 subjects did not report their own use on at least one of the waves. In total, 886 adolescents were included in analyses regarding cigarette smoking, and 938 adolescents regarding alcohol use.

\section{Strategies for Analyses}

In the first stage of analyses a structural model (Figure 4.2) was developed relating subjects, parental and best friends' behavior by longitudinal paths based on theoretical assumptions in which (a) measures of substance use by parents, adolescent and friends were used as predictors of use two and three years later respectively, (b) parental behavior predicts smoking and drinking by adolescents over time and (c) both respondent and friends influence each other over time. Cross-sectional relations between variables were modelled though associations $(\varphi)$ at wave one and residual associations $(\Psi)$ at wave two and wave three instead of causal effects.

Covariance structure models wrere estimated and fitted with LISREL-7 (Jöreskog \& Sörbom, 1989). * Because all variables were ordinal, polychoric rather than Pearson correlations were used as input, and the weighted least squares (WLS) estimation method was used, as suggested by Jöreskog and Sörbom (1989). Preliminary analyses were done with PRELIS to obtain all polychoric correlations and their asymptotic covariance matrix which is needed for WLS estimation.

Model fit was assessed by the following global fit measure: $\chi^{2}$, the NFI (Bentler-Bonnett index), NNFI (Tucker-Lewis index), the root mean square of approximation (RMSEA) and the root mean square residual (RMR). Formally, the $\chi^{2}$ provides a significance test of the null hypothesis that the model is correct. However, $\chi^{2}$ is known to depend strongly upon the sample size $\mathrm{N}$, such that in large samples models may be rejected due to minor specification errors, and in small samples models may be maintained in spite of major misspecification. The NFI and NNFI are much less dependent on $\mathrm{N}$ and generally accepted as descriptive goodness of fit statistics for LISREL models (Bollen, 1989; Jöreskog \& Sörbom, 1993). Both are based upon a comparison of the model $\chi^{2}$ with the $\chi^{2}$ of an independence model that predicts zero correlations. NFI and NNFI should be near 1 , or at least above 
0.90 , for a model to be acceptable. The RMSEA is a measure of model discrepancy $\left(\alpha^{2}\right)$ per degree of freedom, corrected for $\mathrm{N}$, and should be lower than 0.05 . The RMR is a measure of 'average' difference between the observed and fitted covariance matrix. With correlations instead of covariances as input, an RMR below 0.05 seems a reasonable criterion.

If necessary, the initial model was improved with local fit measures. The model could be extended by adding parameters (i.e. causal paths or residual correlations) as suggested by the so-called "modification indices'. The model could be reduced by skipping parameters if these were not statistically significant as suggested by the $T$-tests. Modifications were carried out only if they made sense and the model remained identified.

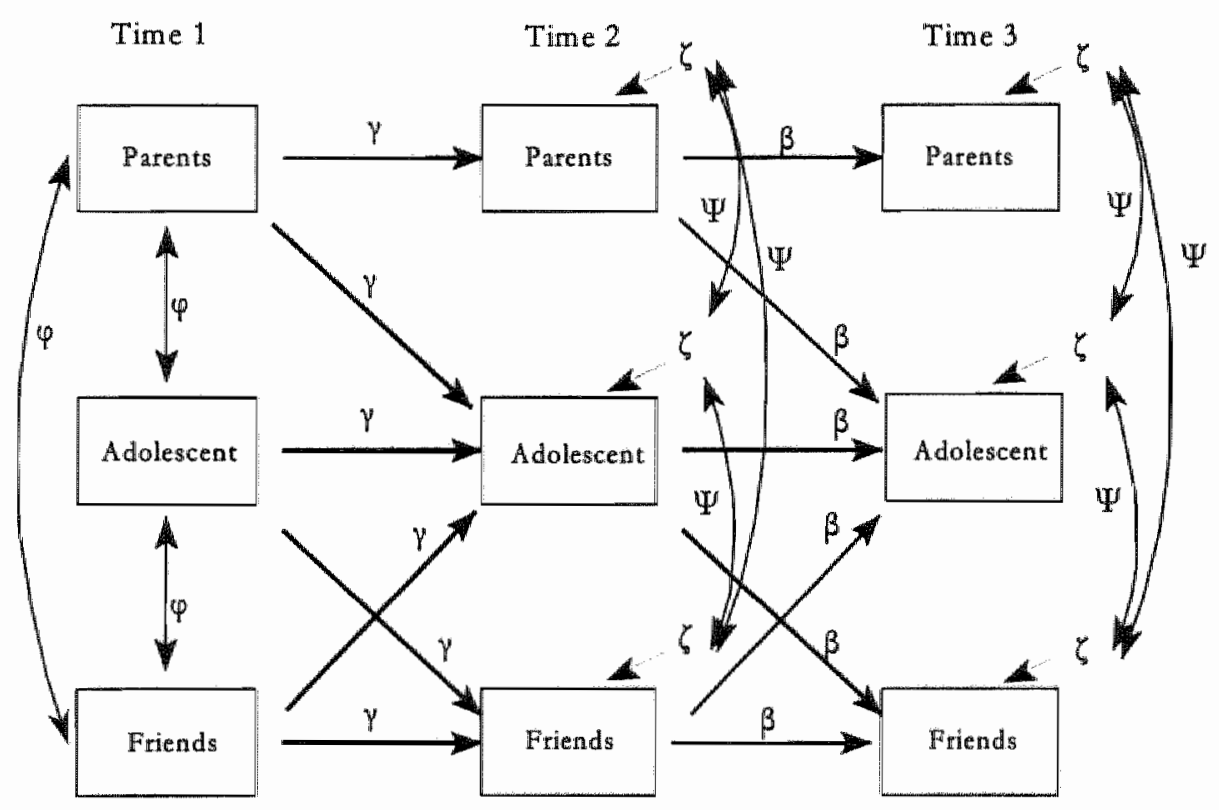

Figure 4.2. Initial Structural Model for Influences of Best Friends and Parents on Adolescent Smoking and Drinking (Standardized coefficients $(\beta)$ are representing longitudinal relations between endogenous variables. Associations between exogenous variables are shown by a ' $\varphi$ ' symbol. Standardized path coefficients between exogenous and endogenous variables are displayed with a ' $\gamma$ '. Residuals of variables are presented with a ' $\zeta$ '. Within time correlations between residuals of endogenous variables are presented by a " $\Psi$ "). 
Table 4.2. Polychoric Correlations, Means and Standard Deviations of Parental, Adolescent and Best Friends' Smoking and Drinking at Three Waves

\begin{tabular}{|c|c|c|c|c|c|c|c|c|c|c|c|}
\hline $\begin{array}{l}\text { Model } \\
\text { variables } \\
\end{array}$ & Mean & $S D$ & 1 & 2 & 3 & 4 & 5 & 6 & 7 & 8 & 9 \\
\hline \multicolumn{12}{|l|}{$\begin{array}{l}\text { varlabies } \\
\text { Cigarente smoking }\end{array}$} \\
\hline Adolescent T1 (1) & 1.72 & 1.20 & 1.00 & .74 & .62 & .21 & .23 & .18 & .59 & .48 & .31 \\
\hline Adolescent T2 (2) & 2.30 & 1.64 & & 1.00 & .79 & .26 & .30 & .28 & .38 & .67 & .43 \\
\hline Adolescent T3 (3) & 3.31 & 2.10 & & & 1.00 & .26 & .26 & .32 & .33 & .51 & .61 \\
\hline Parents T1 (4) & 2.80 & 1.51 & & & & 1.00 & .93 & .88 & .21 & .21 & .24 \\
\hline Parents T2 (5) & 2.73 & 1.61 & & & & & 1.00 & .88 & .19 & .24 & .23 \\
\hline Parents T3 (6) & 2.64 & 1.59 & & & & & & 1.00 & .15 & .19 & .29 \\
\hline Friends T1 ( 7$)$ & 1.69 & 1.12 & & & & & & & 1.00 & .41 & .26 \\
\hline Friends T2 (8) & 2.15 & 1.45 & & & & & & & & 1.00 & .39 \\
\hline \multirow[t]{2}{*}{ Friends T3 (9) } & 2.82 & 1.66 & & & & & & & & & 1.00 \\
\hline & Mean & $S \bar{D}$ & 1 & 2 & 3 & 4 & 5 & 6 & 7 & 8 & 9 \\
\hline \multicolumn{12}{|l|}{ Alcohol use } \\
\hline Adolescent T1 (1) & 2.10 & 1.14 & 1.00 & .57 & .36 & .30 & .22 & .23 & .38 & .25 & .13 \\
\hline Adolescent T2 (2) & 2.85 & 1.26 & & 1.00 & .45 & .24 & .26 & .23 & .33 & .54 & .31 \\
\hline Adolescent "T3 (3) & 4.04 & 1.21 & & & 1.00 & .31 & .36 & .37 & .11 & .18 & .50 \\
\hline Parents T1 (4) & 3.67 & 1.76 & & & & 1.00 & .69 & .62 & .14 & .10 & .14 \\
\hline Parents T2 (5) & 3.86 & 1.74 & & & & & 1.00 & .72 & .01 & .12 & .20 \\
\hline Parents T3 $(6)$ & 3.80 & 1.82 & & & & & & 1.00 & .01 & .05 & .27 \\
\hline Friends 'T'1 ( & 1.28 & .78 & & & & & & & 1.00 & .38 & .18 \\
\hline Friends T2 (8) & 1.91 & 1.30 & & & & & & & & 1.00 & .22 \\
\hline Friends T3 (9) & 3.34 & 1.60 & & & & & & & & & 1.00 \\
\hline
\end{tabular}

\section{RESULTS}

Developments of Smoking and Drinking by Parents, Friends and Adolescents: Means and Correlates

Table 4.2 presents the adolescents' reports of their own smoking and alcohol use, and perceived behavior of parents and peers presented by means and standard deviations. In addition, cross-sectional and longitudinal associations between all variables are depicted. The amount of smoking increased during adolescence. In 1989 prevalence of regular smoking was quite low: $2.3 \%$ were daily smokers. Two years later, a proportion of $10.1 \%$ reported smoking daily. Five years after the first measurement, nearly a third of the sample $(30.5 \%)$ smoked on a daily basis. Similar patterns were found when using the sum scores of the index describing smoking (on a 6-point scale): $M=1.72$ at the first wave, $M=2.30$ at the second wave, and $M=$ 3.31 at the last wave. Weekly uptake of drinking progressed rapidly during adolescence. Only $2.9 \%$ were weekly drinkers at the first measurement. Two years later, $9.3 \%$ of the subjects reported that they drank at least weekly. In 1994, 
approximately half of the respondent group (49.3\%) indicated drinking weekly. A similar pattern was displayed for the index of adolescent' alcohol use with means of 2.10 (1989), 2.85 (1991) and 4.04 (1994).

Both parental and best friends' substance use were, in all cases, positively related to use by subjects. In general, considering the magnitude of coefficients, adolescent behawior appeared to be more strongly associated with friends' use than parental use. At a transversal level, associations of use by parents and their offspring were somewhat stronger with respect to alcohol than cigarette use. Coefficients varied from .21 to .32 for smoking, and .26 to .37 for drinking. High correlations at each wave supported assumptions about homogeneity of smoking and drinking in friendships. For smoking, correlates ranged from .59 to 67 . Associations indicating similarities in alcohol use were slightly smaller than for smoking but nevertheless substantial (estimates between .38 and .54).

\section{Structural Madel of Influences of Peers and Parents on Cigarette Smoking}

An initial model was estimated with all pathways depicted in Figure 4.2. This initial model fitted the data very well: $\chi^{2}(886)=23.21, p=.08, \mathrm{df}=15, \mathrm{NFI}=1.0, \mathrm{NNFI}$ $=1.0, \mathrm{RMSEA}=.03, \mathrm{RMR}=.03$. Examination of the modification indices showed that the model fit could be further improved by freeing two paths, one path between parental smoking at T1 and friends' smoking at T2, and one path between parental smoking at T2 and friends' smoking at T3. Freeing these parameters resulted in excellent fit of this model with statistics: $\chi^{2}(886)=5.79, p=.95, \mathrm{df}=13$, NFI $=1.0$, NNFI $=1.0$, RMSEA $=.00$, RMR $=.02$. The modification indices suggested no further model extensions. Model reduction was done by testing each free parameter for significance with $\alpha=0.10$ instead of 0.05 to avoid type II errors. Three non-significant paths were excluded from the initial model resulting in a well fitting model: $\chi^{2}(886)=9.25, p=.90, \mathrm{df}=16, \mathrm{NFI}=1.0, \mathrm{NNFI}=1.0, \mathrm{RMSEA}=$ $0.00, \mathrm{RMR}=.02$. Standardized parameter estimates obtained from the final model are displayed in Figure 4.3.

Model parameters show that smoking by subjects is strongly predicted by previous behavior with path coefficients of .710 and .801 . The final model suggests that individual smoking can be more accurately predicted by parental smoking than best friends' smoking. One cross-lagged longitudinal path from parental to adolescent behavior was displayed (T1-T2, .130). Thus, youngsters with a smoking family background at wave two were more likely to develop smoking habits themselves over a period of two years. We found no support for longitudinal effects of two best friends' smoking on adolescents use. On the contrary, the extent to which an adolescent has friends who smoke is predicted by prior smoking of the respondent. Standardized coefficients for impact of subject smoking at time 1 on friends' smoking at time 2 were .324 , and for adolescent use at time 2 on friends' smoking at time $3, .269$. 
Time 1

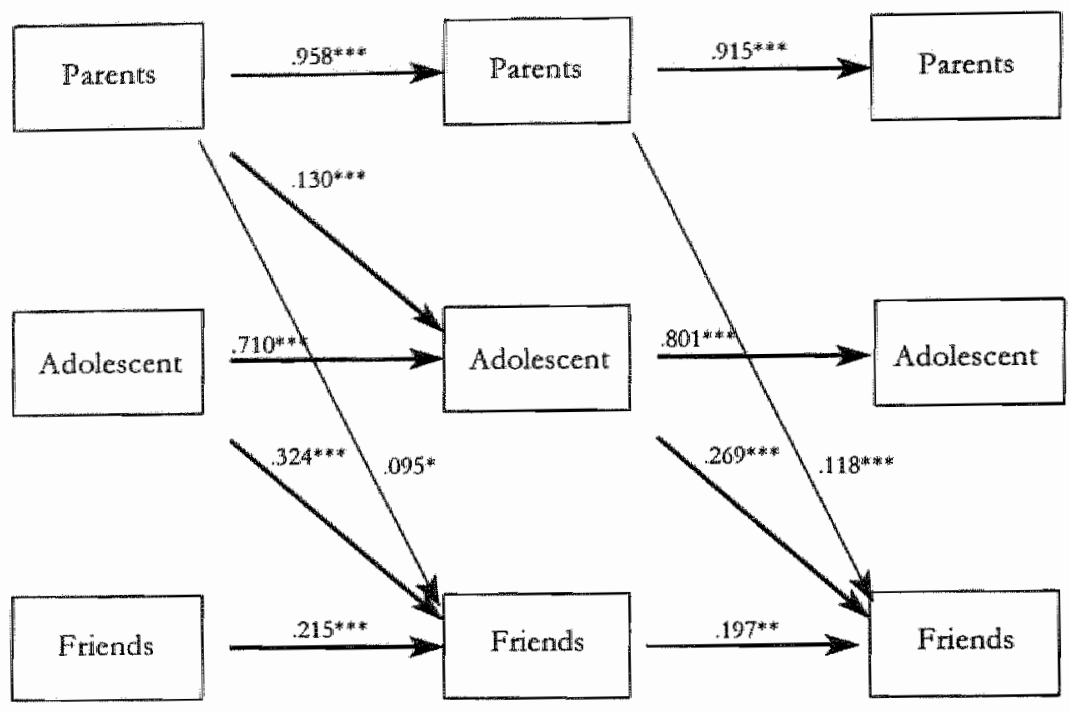

Figure 4.3. Structural Model for Impact of Best Friends and Parents on Adolescent Smoking (Significant values: ${ }^{*} p<.05,{ }^{* * *} p<.01, * * * p<.001$ )

Other interesting outcomes, besides the hypothesized effects, can be mentioned. The effects of previous behavior were strong for parental smoking with coefficients of .958 and .915 . For friends' smoking, however, the effects were less convincing (estimates of .215 and .197 ). The polychoric correlations showed that associations of friends' smoking over time were not as high as parental and adolescent smoking. As subjects were asked to report their current best friends behavior, low associations of friends' smoking over time may be due to changes in referent persons rather than behavioral inconsistency. Furthermore, parents seem to exert an influence on behavior of friends over time. Perceived smoking by parents predicted higher levels of use by best friends with structural coefficients of .095 and .118 .

With respect to other significant parameters, parental alcohol use appeared to be the most stable behavior (estimates of .695 and .552) with a direct path between time 1 and time 3 (.246). Smaller effects of previous drinking were found for friends, with a coefficient of .339 (T1-> T2) and a non-significant path ( $p$ between .05 and .10) between time two and three. Similar to our results for smoking, a significant path from parental alcohol use to friends' drinking was found (T2->T3, $\beta=.074$ ). 
Structural Model of Influences of Peers and Parents on Alcobol Use

As explained before, an identical model was postulated to examine changes in alcohol use by influences of perceived behavior of parents and peers. The initial model provided a fair fit: $\chi^{2}(938)=38.15, p<.01, \mathrm{df}=15, \mathrm{NFI}=99, \mathrm{NNFI}=$ $99, \mathrm{RMSEA}=0.04, \mathrm{RMR}=.03$. Inspection of the modification indices showed that two paths might be added to the initial model, that is, a path between parental use at 'T2 and friends' use at T3, and the association between parental alcohol use at $\mathrm{T} 1$ and T3. This resulted in an excellent fit: $\chi^{2}(938)=12.83, p=.46, \mathrm{df}=13, \mathrm{NFI}$ $=1.0, \mathrm{NNFI}=1.0, \mathrm{RMSEA}=0.00, \mathrm{RMSR}=.02$. There were no non-significant paths $(p>.1)$ which could be excluded from the adjusted model. 'Thus, the initial model with two additional paths became our final model (Figure 4.4).

The results show that by far the strongest predictor of alcohol use by adolescents was previous behavior. Adolescents' drinking at T2 and T3 was predicted by their own drinking at the previous time point, with standardized regression weights of .503 and .479 respectively. Over a period of two and three years alcohol use by parents fostered higher frequency of use by their children. Estimation parameters were .083 at T2 and .262 at T3. In early adolescence, friends

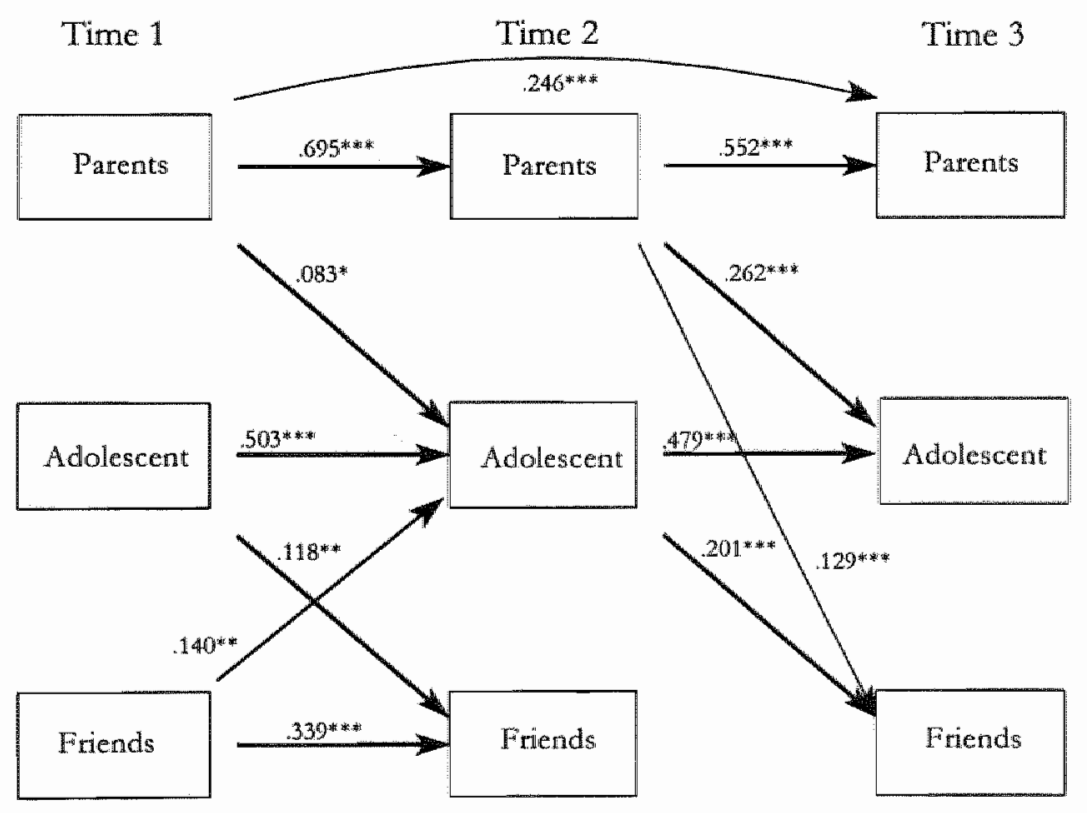

Figure 4.4. Structural Model for Impact of Best Friends and Parents on Adolescent Alcohol Use (Significant values: ${ }^{*} p<.05,{ }^{* * 4} p<.01$, ${ }^{* 4 * * t} p<.001$ ) 
exerted an impact on subject use (T1- $\left.>T^{2} 2 ; \beta=.140\right)$, but this effect disappeared three years later $(\mathrm{T} 3)$. On the other hand, an impact of adolescents on friends was found at both time points with path coefficients of .118 and 201.

\section{The Effects of Best Friends' Use for Subjects Without Using Parents: A Further Analysis}

In order to examine whether parental use precluded possible influence of friends, additional analyses were performed with reciprocal cross-lagged effects of friends' and adolescents' smoking or drinking at the three time points for the selection of youth with non-using parents at wave one. ${ }^{5}$

A model was evaluated for those subjects who had no smoking parents $(n=$ 344). The initial model was constructed according to Figure 4.2, except that parental use was left out of the model. An initial model was tested which fitted the data well: $\chi^{2}(344)=2.57, p=.63, \mathrm{df}=4, \mathrm{NFI}=1.0, \mathrm{NNFI}=1.0, \mathrm{RMSEA}=.00, \mathrm{RMR}=$ .03. After deleting four non-significant paths a final model was obtained: $\chi^{2}(344)=$ $4.16, p=.84, \mathrm{df}=8, \mathrm{NFI}=1.0, \mathrm{NNFI}=1.0, \mathrm{RMSEA}=.00, \mathrm{RMR}=.03$. Best friends' use was not predictive of adolescent use at the following time point, but adolescent smoking was predictive of best friends' use at the following time point with path coefficients of respectively .503 and .502 .

For the analyses with respect to drinking, we selected those subjects with no regular drinking parents $(n=409)$, since non-use of both parents hardly occurred. An initial model was tested: $\chi^{2}(409)=5.05, p=.28$, df $=4$, NFI $=.99$, NNFI $=$ $1.0, \mathrm{RMSEA}=.03, \mathrm{RMR}=.04$. A final model was estimated after deleting three non-significant paths: $\chi^{2}(409)=6.86, p=.44, \mathrm{df}=7, \mathrm{NFI}=.99, \mathrm{NNFI}=1.0$, RMSEA $=.00, R M R=.05$. This model showed that best friends' alcohol use was influenced over time by subjects ${ }^{x}$ use, with coefficients of .166 and .385 . No impact of friends' use on subjects' alcohol use was found. ${ }^{6}$

\section{DISCUSSION}

The present study examined the relative influence of smoking and drinking behavior of two best friends and parents on adolescents' substance use. Prospective associations were compared by means of structural equation modeling. Overall, proposed models fitted our data well, although this fit could be improved by a few model modifications.

Transwersal correlations between parental smoking and drinking and adolescent use were moderate. In the long run, influences of parents on their children were small but consistent. Youngsters were affected by exposure to "using" family members with regard to specific behavior and stage of adolescence. For instance, alcohol use by parents fostered drinking by their offspring at a later age. Only for smoking, was no effect apparent of parental smoking at the second wave on adolescent smoking at the third wave. 
Young people tended to associate with similar others. High cross-sectional associations, between .38 and .67 , emphasized the importance of friendships with respect to smoking and drinking. In this study, however, homogeneity of smoking and drinking in friendships could hardly be explained by the influences of the two best friends. Only in one of four equations, was a significant path found between friends' use (drinking, time 1) and adolescent use (time 2). In contrast, cross-lagged effects of adolescent use on peer use were significant for smoking and drinking at wave two and three. Thus, instead of the hypothesized longitudinal impact of smoking or drinking friends, our findings suggest that adolescents themselves are for a greater part accountable for homogeneity of behavior in friendships.

The present study shows that prior experiences with substances are generally robust in predicting use a few years later. In agreement with the hypotheses, adolescents who reported earlier alcohol or cigarette use at time 1 and time 2 were more likely to maintain or increase their use at following measurements. Continuity of smoking and drinking behavior by young people was confirmed in previous studies (e.g., Fergusson et al., 1995; Stacy et al., 1994).

The assumption that the effects of smoking and drinking friends are more prominent when adolescents grow up within a non-using family background was tested in additional analyses. For those youngsters without using family members, there were no effects of friends' use on subsequent adolescent smoking and drinking. In general, these results strengthen the generalization of aforementioned outcomes.

\section{Parental Influences}

In agreement with the social learning theory of Bandura (1977; 1986) support was found for the modeling effects of exposure to smoking and drinking parents. The results are in contrast with studies which point out that individual substance use is primarily determined by peers or specific friends (e.g., Ary $8 x$ Biglan, 1988; Aseltine, 1995). Besides a different analytic perspective compared to most studies (e.g., use of structural modeling, and reciprocal paths between friends" and subjects' use), explanations for the apparently more prominent role of parents might also be owing to cultural differences. Differences in family structure, life-style and socialization processes might be accountable for cross-cultural variation in parental influences.

Unexpectedly, direct paths between parental use and friends' smoking and drinking were displayed. This finding is congruent with studies by Hansen, Graham, Sobel, Shelton \& Flay (1987) and Allegrante, O'Rourke and Tuncalp (1977) who stated that the selection of friends was mediated or influenced by parents. One of the postulated explanations is that '... parents may act as friendship formation gatekeepers' (Hansen et al., 1987: 577). Parents who do not smoke may be more active in prohibiting their children smoking by exerting influence on 
friendship choices. Internalization of a family life-style and socialization processes might also be responsible for this association. Moreover, the role of the two closest friends of adolescents was investigated and not the overall peer group. When friends visit adolescents" parental homes, parents might have an impact by functioning as role models, or offering them alcohol beverages or cigarettes. Further research is necessary to unravel particular mechanisms of family influences.?

\section{Influences of Friends}

Perception of best friends" substance use had virtually no effect on respondents' behavior over time. Before we exclude peer influences as a major factor in predicting adolescent substance use, some aspects must be taken into consideration. First, this study did not focus on different stages of substance use. Another analytic strategy is required to examine the impact of peers on the onset of smoking and drinking. However, in another study we examined the effects of peer group smoking, best friend smoking and parental smoking on the transition from nonsmoking to smoking using the same data-set. Logistic regression analyses revealed that peer group smoking as well as best friend smoking did not affect smoking onset at wave two and wave three (Engels, Knibbe \& Drop, 1998b). Furthermore, we included the use of two best friends as an indicator of peer influences. Although it is assumed that these persons are most relevant for individual behavior (Urberg et al., 1997), results might be different when the smoking or drinking status of the overall peer group or the schoolmates are included.

Overall, our results are counter to considerations of Conrad et al. (1992) in their review of longitudinal studies. Most of their reviewed studies, however, did not include a test of the relation of adolescents' use with the tobacco use of friends later on. The few studies which did include the possibility of selective association as an explanation for homogeneity in friendships concluded that when selection processes were taken into account less variance of behavioral similarities in friendships could be attributed to the fostering effects of friends (Bauman \& Ennett, 1996; Billy \& Udry, 1985; Ennett \& Bauman, 1994; Kandel, 1978). In the present research, reciprocal paths between friends and adolescents were incorporated to acknowledge the role of adolescents themselves in friendships. However, in our study, it was not possible to determine whether the impact of adolescents on friends' behaviors could be entirely attributed to selective association. Specific information about changes in relationships with best friends is required to delineate adlaption and selection processes from the subjects perspective. Nonetheless, information was available on the differences in peer group constellation between the second and third wave. Analyses on these data showed that homogeneity of smoking behavior was not affected by influence processes. In stable peer groups, no impact of smoking peers on the onset of adolescent smoking was observed. Strong 
support, however, was found for selection effects (Engels, Knibbe, Drop \& De Haan, 1997). An argument against interpretation of the influence of adolescents on their friends" behavior in terms of peer influence is the fact that a substantial proportion of the adolescents reported that their peer group changed considerably (Engels, Knibbe, Drop \& De Haan, 1997). Nearly 45 percent (of 1,063 subjects) indicated that more than half of their peer group consisted of new social contacts in a period of three years. Furthermore, if the impact of adolescents on their friends was interpreted as actual influence this would lead to the conclusion that our sample comprised an over representation of adolescents with friends who were likely to adopt their smoking and drinking behavior. However, the sampling procedure (i.e., entire classrooms were included in the study) makes this very unlikely. Finally, the correlations between friends" substance use over time are relatively low. It is plausible that not the individual behavior is unstable, but that we deal with different referent persons.

One may question to what extent the absence of longitudinal influences by friends leads to the conclusion that peer influences are not apparent. Considering the years between measurements (two and three years, respectively) it is possible that friends who actually influenced the respondent did not belong to the "two best friends' which were reported at each wave. This is especially so if one takes into account that many friendships changed during the study. More accurate information may be gained by using shorter intervals between waves through which mutual reinforcing processes within steady friendships can be observed. Nevertheless, it should be mentioned that studies with shorter follow-up surveys (i.e., one year) found also only limited support for peer influences, even when a differentiation was made between steady and unsteady friendships (e.g., Ennett \& Bauman, 1994).

\section{Limitations of the Study}

Although the fit of the model is good for the total sample, subsamples may differ in the model explaining adolescent smoking and drinking. For example, it is feasible that the pathways to substance use are dissimilar for girls and boys. Variations in education, urbanization, neighbourhood, and religious affiliation could also result in other effects of friends and parents.

To be more precise about the relative contribution of selection and influence processes to similarities in peer groups, detailed data is needed on the changes in friendships between two or more waves. Moreover, it would be better if a social network was included in the study. In that case, one can investigate whether friendships are reciprocal at the level of best friends and the peer group. Sociograms can be constructed which provide information on dyads, cliques and loners. Another advantage is that data on actual friends' use can be obtained instead of perception-data. Although some studies used a network approach (see Ennett \& 
Bauman, 1994; Urberg et al., 1997), in our opinion, insight in social interaction processes would be enhanced if parents and siblings are also included. Then questions about, for example, the impact of parents on friendships choices and the relative impact of siblings and friends, could be answered.

Besides friends and parents, siblings and other adults could play a significant role in the development of substance use. Other studies have already shown the relevance of mainly older siblings at initiation of smoking and drinking (e.g., Conrad et al., 1992; Needle et al., 1986). Measures of substance use by older siblings could not be included in analyses. In further research it would be interesting to examine the role of siblings with respect to interactions within families.

This paper focuses on the direct impact of perceived behavior of significant others on individual behavior. Some researchers have postulated that the perceived behavior of others only indirectly fosters substance use. According to the extended theory of reasoned behavior, behavior is exclusively determined by adolescents' intentions, substance-specific beliefs, perceptions of social norms, and self-efficacy (Ajzen, 1991; De Vries, Dijkstra \& Kuhlman, 1988). Nevertheless, some empirical studies show the unique contribution of perceived behavior in addition to these concepts, featuring the exposure to 'using' significant others as a direct predictor of behavior (De Vries, Backbier, Kok \& Dijkstra, 1995; Flay et al., 1994). Furthermore, it is conceivable that influences from friends do not only operate by modeling processes. Pressure or explicit offers are not only related to the behavior of friends, but demonstrate a unique contribution to the initiation of youngsters' substance use (Evans et al., 1978; Graham et 2l., 1991). Further research is warranted to compare different processes of social influences on adolescent substance use.

The present study relied on the self-reports of adolescents about their own smoking and drinking, and use by parents and peers. Confidentiality was assured to obtain accurate reports of subjects' own use. This is one of the ways of obtaining reliable and valid responses (Botvin \& Botvin, 1992). Moreover, it is possible that the perception of alcohol and cigarette use is affected by ones own substance use (Marks \&x Miller. 1987). However, when independent reports of parents, peers and adolescents were obtained, high correlations indicated that young people were well aware of drinking by significant others (Wilks et al., 1989). In addition, we assume that people are better able in the recall of their best friends use than the behavioral patterns of their peer group or school mates (e.g., or to give a precise estimate of the number of friends who smoke or drink). It remains a question whether our findings are profoundly distorted by potential problems with self-reports. According to Urberg et al. (1997) and Hu et al. (1996) predicting future behavior on the basis of adolescents' perceptions of their friends behavior is, to an extent, predicting future behavior on the basis of adolescents' present behavior and produces more evidence of influence than use of the friends ${ }^{3}$ actual behavior. Thus, if we found strong evidence of peer influences on adolescent use in particular, we should be cautious because of the use of perception data. 
Finally, the findings of this study may have some implications for the development of prevention programs. Until now, many programs have been designed to improve resistance skills and make youth aware of peer influences. The limited support for peer influences in our study may lead to a reconsideration of the content of these programs. In our opinion, two aspects deserve some attention. First, the strong effects of previous behavior on substance use lead to the conclusion that young people must be approached at early stages of initiation. Secondly, the fact that parents do play a small but significant role in the development of smoking and drinking habits stresses the importance of family factors into health promotion programs. For example, community based programs directed at both parents and their children might result in a lower uptake among adolescents, higher quitting rates among adults and a reduction of role models. Leventhal, Keeshan, Baker and Wetter (1991) suggest to enhance the dialogue between adolescents, schoolmates, friends and parent which might facilitate a better understanding for youngsters of their decisions, influences and feelings.

\section{NOTES}

\section{${ }^{1}$ Based on:}

Engels, R.C.M.E., Knibbe, R.A., De Vries, H., Drop, M.J., \& Van Breukelen, G.J.P. (1998). Influences of parental and best friends' smoking and drinking on adolescent use: A longitudinal study. Journal of Applied Social Psychology (in press).

${ }^{2}$ Two main types of secondary education can be distinguished in the Netherlands: vocational schools preparing students for jobs like electrician, carpenter or housewife, and high schools for continued and more specialized education at colleges and universities.

${ }^{3}$ Separate attrition analyses were conducted for the samples used in structural equation modelling. Regarding cigarette smoking, dropouts $(n=568)$ were more likely to be male, older, lower educated, went less often to church and had less ofteri a father from Dutch origin compared to remainers $(n=886)$. Also significant differences were found on frequency of smoking $(\mathrm{OR}=1.17,95 \% \mathrm{CI}=1.09-1.26)$. No differences were found for frequency and intensity of alcohol use, and intensity of cigarette smoking. For alcohol use, drop outs $(n=516)$ were more likely to be male, older, lower educated, went less often to church, and had less often an father from Dutch origin. They also consumed alcohol more frequently $(O R=1.25$, $95 \% \mathrm{Cl}=1.17-1.38)$ and consumed more glasses per week $(\mathrm{OR}=96,95 \% \mathrm{CI}=.91-1.01)$ compared to remainers $(n=938)$.

"No multiple indicators were awailable in this study. However, there are other reasons for using LISREL here. First, LISREL allows residuals (prediction errors) of different variables to be correlated. Such correlations are typically found between variables measured at the same time (e.g. adolescent and friends use). One might replace such correlations by causal paths, of course, but their direction would be arbitrary. Second, LISREL not only gives the $\mathrm{R}^{2}$ per dependent variable, but also overall model fit measures and indices where the model needs to be modified. These are indispensable for comparing and improving models. Third, since our variables were measured on ordinal instead of continuous scales, Pearson correlations and 


\section{Chapter 4}

analyses based there upon (like path analysis) can be misleading due to non-normality and heterogeneity of distributions (some variables positively skewed, others not etc.). LISREL provides a solution to this by computing polychoric correlations and estimating the model with a special method called WLS.

${ }^{5}$ Only the main findings of this hypothesis are presented in this paper. An extensive report of the analyses and the polychoric correlation matrices is available from the first author.

"We also performed analyses for subjects who had at least one smoking parent $(n=542)$ and for subjects with at least one regular drinking parent (daily or weekly, $n=529$ ). Borh initial models provided a good fit. In general, the findings were comparable with those for non-using parents - non-significant effects were found for friends' use on adolescent use and significant effects were found for adolescent use on friends' use.

${ }^{7}$ It could be argued that adolescents have some effect on the drinking and smoking habits of their parents. For example, it is often mentioned that a side effect of health promotion activities at school consists of non-smoking children trying to persuade their parents to quit smoking. Or, on the other hand, when adolescents start to drink more frequently at home, their parents might join them resulting in higher consumption levels. However, in the current study, the high correlations of parental smoking and drinking over time, and the fact that modification indices did not suggest additional paths from adolescent use to parental use over time, do not point to a strong impact of children on parental use. 


\section{5}

\section{INTIMATE RELATIONSHIPS AND ALCOHOL USE: WHEN LOVE COMES TO TOWN ${ }^{1,2}$}

During adolescence, social relationships are open to change. In early adolescence, children focus primarily on their parents to fulfill their personal needs (Douvan \& Adelson, 1966; Kelly \& Hansen, 1987; Moore, 1987). Over the years, adolescents' concern of achieving intimacy goals, such as closeness and trust, refocus on activities concentrating on the company of peers. According to Erikson (1958) two main goals exist in the period of late adolescence, namely, separating from parents and making a commitment to one's own identity. It is therefore important to associate with new peers or strengthen existing bonds; in this way one can reflect one's own ideas, opinions and emotional development (e.g., Paul \& White, 1990). Adolescence is also a period of love and romance - teenagers become interested in dating and sexual experiences, and have their first intimate relationship (Sanderson \& Cantor, 1995). Although adolescent relationships are in many cases sequential and short in duration, researchers have stressed the facilitating and influential effects of these relationships on teenagers' well-being and communicative skills (Paul \& White, 1990; Samet \& Kelly, 1987; Weinstein \& Rosen, 1991). The impact of intimate relationships on the personal and social development of adolescents draws attention to the associations between romantic relationships and substance use patterns. Recent literature on alcohol and dating or partnership among adolescents has concentrated mostly on the effects of alcohol consumption on violence and sexual abuse in dating situations (e.g., Gagne \& Lavoie, 1993; Williams \& Smith, 1994) or on the use of contraceptives (e.g., Leigh, Schafer \& Temple, 1995; McLean \& Flanigan, 1993). The present study explores the associations between involvement in a steady relationship and adolescents' alcohol use using longitudinal data of 14 to 
18 year-olds. First, the effects of alcohol use on the formation of steadly relationships were examined. Second, the possible tendency of young people to change their drinking habits once they became engaged in a romantic relationship was exmined.

For the development of prevention programs, it is considered important to focus on the relationships between these two phenomena. Recent research has suggested that (moderate) alcohol use could be functional to young adolescents in terms of peer relationships and personal development (Leifman et al., 1995; Pape \& Hammer, 1996). If alcohol use appeared to be positively related to partnership, health education and policy elforts preventing youngsters drinking might have very limited effects if the personal and social benefits of drinking were ignored. Furthermore, despite the significance of intimate relationships for adolescents, prevention programs have hardly acknowledged possible effects of a partner on the development of drinking habits. If research demonstrated that partnerships were of influence, more effective programs could be developed.

\section{Alcobol Consumption and the Formation of Steady Relationships}

Does drinking increase or decrease the chances of involvement in romantic relationships? One could argue for a positive relationship between drinking and partnership. Drinking in adolescence could be part of a life style which involves going out to pubs and discos, spending time with friends, less control by parents, and also first experiences with intimate relationships. There is some empirical evidence for this assumption. In a longitudinal study of 19 to 22 year-olds, Pape and Hammer (1996) found that those who experienced their first intoxication late, or were abstainers were less likely to have a steady partner. The mechanism behind this positive association might be sought in the setting in which adolescents consume alcohol. Adolescents" alcohol consumption is concentrated in public drinking places and at parties in most Western countries (Harford \& Grant, 1987; Knibbe, Oostveen, Van de Goor, 1991; Weiss \& Moore, 1994). Silbereisen, Noack and Von Eye (1992) showed that when youngsters are trying to establish a relationship or are looking for a romantic affair, they go to places that provide this possibility. Since their research on Berlin teenagers showed that discos and bars are settings which serve the purpose of a "marriage market" for many adolescents, it is likely that drinking is positively associated with partnership. For youngsters in the Netherlands, going to social settings such as bars, discos and parties is also an important leisure activity (De Zwart, Warnaar \& Van Dam, 1994; Engels, Knibbe \& Drop, 1998a). At the ages of 17 to 19 , they spend about eight hours per week in public drinking places or parties. Particularly those who drink in public drinking places and at parties have more opportunities to meet potential partners and to become involved in a steady relationship. On the contrary, it is unlikely that drinking in the company of parents or drinking solitarily improves one's chances to become involved in a relationship. 
However, one could also argue that alcohol consumption by adolescents is most likely negatively related to the formation of a steady relationship. A few studies on drug use and the occupancy of young adult social roles (e.g., employment, marriage) have suggested that drug use and partnership are incompatible (Chassin, Presson, Sherman \& Edwards, 1992; Newcomb \& Bentler, 1988; Yamaguchi \& Kandel, 1985). The rationale behind a negative relationship is twofold. First, drug use could be perceived as a less attractive feature for potential partners. Particularly so if substance use is imbedded in a cluster of deviant behaviors and values, such as truancy, rebelliousness, delinquency, and the use of other illicit drugs. Drinking could be part of a way of life which does not appeal to others. Second, drug use could cause impairment that makes it difficult for individuals to maintain their relationships (Chassin et al., 1992). It is difficullt to extrapolate the findings on drug use to alcohol. In contrast to illegal drugs, the use of alcohol is widely accepted (and almost normative) in most if not all European countries. Nevertheless, it is still possible that high levels of alcohol use and frequently being drunk in middle adolescence is perceived as deviant and negatively related to the formation of a steady relationship.

\section{The Impact of Partnership on Changes in Alcobol Use}

Once young people become engaged in a romantic relationship, they might change their drinking habits. We would expect a decrease in alcohol consumption. To the best of our knowledge, no studies have investigated this issue in an adolescent sample. The literature on adults, however, offers some insights into the links between relationship formation (marriage, cohabiting) and changes in alcohol consumption (e.g., Hanna, Faden \& Harford, 1993; Temple et al., 1991). Most studies have reported that partnership moderates drinking. For instance, a thorough study among young adults by Power and Estaugh (1990) showed that relationship formation was associated with lower levels of alcohol use, while disruption of the relationship was associated with higher levels of alcohol use. There are some explanations for these effects. First, involvement in a steady relationship might activate specific norms and expectations of how people could and should act in relationships (i.e., which subsequently affect drinking behavior). In addition, partnership implies changes in activities and in the distribution of leisure time - people spend more time with their partners in private settings (at home or at a friend's home) and less time in public drinking places (Knibbe, Drop \& Muytjens, 1987).

When generalizing these results to adolescents, two crucial differences are worth mentioning. Marrying or cohabiting in (young) adulthood, or having your first partner in adolescence are two different worlds. Perhaps the mechanisms proposed for the impact of partnership on drinking patterns by adults might not be suitable for adolescents. Furthermore, there is a very strong increase in alcohol consumption during the adolescent years in the Netherlands (Kuipers, Mensink \& 
De Zwart, 1993) and in other Western countries (Johnston, O'Malley \& Bachman, 1996 , Plant \& Foster, 1991). Therefore, if partnership is of influence at these ages, it is more realistic to expect that partnership will negatively influence the rate of increase of alcohol use than that it will cause a decrease in consumption levels.

\section{The Present Study}

This study aims to explore the associations between alcohol consumption and partnership among adolescents. Data from a nationwide three-wave longitudinal study among children at secondary school were used for analyses. Two main hypotheses were tested in this study. First, we tested the hypothesis that higher consumption of alcohol at the age of 14 or 15 (wave two) would be positively related to partnership three years later (wave three). In addition, it was examined whether the likelihood of becoming involved depended on the context of drinking. Drinking at home was hypothesized not to be related to partnership formation while drinking in peer settings was expected to be related to partnership. Second, it was investigated to what extent changes in alcohol consumption between the two waves were related to partnership. Adolescents involved in a relationship were expected to report a smaller increase in alcohol use than those who were not involved. Because of the robust differences in alcohol use between boys and girls, separate analyses were conducted. Regarding the research questions, no gender differences were hypothesized.

\section{METHOD}

\section{Procedure}

Data for this article were derived from a three-wave longitudinal study on the predictors of smoking and drinking behavior among secondary school children in the Netherlands. The study started in 1989 with a sample of 1,454 students from first and second grade. Thirty-six schools differentiated by educational level and region cooperated. At the first measurement, the questionnaires were administered in the classrooms. In the autumn of 1991 and 1994, forms were sent to subjects" homes. Participants not responding to two postal reminders were contacted by phone to complete an interview. The response rates were $82 \%$ at both follow-up surveys. A total of 1,063 adolescents $(73 \%$ ) were enrolled in all three waves. Because the telephone interview at the third wave contained only a limited set of items, the sample was restricted to those who filled out the questionnaire $(n=958)$. To determine the nature of sample attrition, a logistic regression analysis was conducted which showed no significant differences $(p>.05)$ on frequency and intensity of smoking and drinking between drop-outs and respondents. An extensive description of the attrition analyses is reported in Chapter 4. 


\section{Measures}

The main analyses were restricted to data from the second (mean age of the respondents 14.4) and third wave (mean age 17.4) with an interval of three years. At both waves, subjects were asked whether they had consumed alcohol during the previous six months ( 1 'yes', 2 'no'), and what the frequency of drinking in the preceding four weeks was ( 1 'not in the last four weeks', 2 'in the last four weeks but not in the last week', 3 'every week but not daily', and 4 'daily'). In addition, the frequency of use was reported for four situations: at home, at parties and special gatherings, at a friend's home and in public drinking places. The responses ranged from 1 'not in the last four weeks' to 4 'daily'. A quantitative measurement of alcohol use was obtained by adding the number of glasses they consumed during weekdays and the weekend of the preceding week. A distinction was made between weekly consumption at home and weekly consumption in public drinking places. The frequency of drunkenness assessed at wave two ranged from 1 'not in the last two years' to 7 'more that 20 times'. At wave three, subjects were asked whether they had been involved in a steady relationship during the previous three years. ${ }^{3}$ The response categories were 1 'Yes, I have a steady partner at the moment', 2 'No, I have had a partner in the last three years but not any more', and 3 'No, I have not had a partner in the last three years'.

\section{Data Analyses}

We restricted ourselves to respondents in the age group of 14 to 18 year-olds (wave two and three) since the central focus of our study concerned the role of a steady partner. The numbers of respondents who were involved in a romantic relationship were too small at the first wave (mean age 12.4). Furthermore, it is important to note that we consistently differentiated three categories of adolescents: youngsters with a steady partner, youngsters who were not involved in an intimate relationship at the moment of measurement but reported having had a partner in the preceding three years and youngsters with no experience of a steady relationship.

First, univariate analyses were conducted to examine the scores on the alcohol measures: frequency of drinking, frequency of drunkenness and level of weekly consumption at we two for the three categories. Subjects with at least one missing value on the variables employed were omitted from the subsequent analyses. Second, the frequency of drinking in four drinking situations (i.e., at home, at a friend's place, at parties and special gatherings, and in public drinking places) at wave two were related to the three partnership categories by univariate analyses. All these analyses were performed for men and women separately. The extent to which people's involvement in a steady relationship was associated with changes in drinking behavior between wave two and three was examined by univariate analyses. The changes in the total weekly consumption, weekly consumption at home and weekly 
consumption in public drinking places were calculated. To provide insight into the relative increase in consumption for the three partnership categories, the consumption levels for ave two and three are also presented. Since the range of the distribution of age within the sample was quite limited, no control for age was conducted.

Table 5.1. Longitudinal Relationships between Drinking Variables at Wave Two and Partnership at Wave Three

\begin{tabular}{|c|c|c|c|c|}
\hline & $\begin{array}{l}\text { Having a } \\
\text { partner }\end{array}$ & $\begin{array}{c}\text { Currently } \\
\text { not involved }\end{array}$ & $\begin{array}{c}\text { Never had a } \\
\text { partner }\end{array}$ & F-value \\
\hline \multicolumn{5}{|l|}{ Males } \\
\hline Freq drinking $(1-4)^{2}$ & $(.75)$ & $(.75)$ & (.63) & $7.66^{\text {나내 }}$ \\
\hline Freq. drunkenness $(1-7)^{b}$ & $1.76 \quad(1.43)$ & $1.33(1.04)$ & $(.93)$ & $8.45^{* 254}$ \\
\hline Consumption levele & $3.44(7.84)$ & $2.15 \quad(6.15)$ & $.95 \quad(3.23)$ & $7.79^{* 44}$ \\
\hline \multicolumn{5}{|l|}{ Females } \\
\hline Freq. drinking & $(.64)$ & $(.63)$ & $(.52)$ & $6.01^{* 4}$ \\
\hline Freq. drunkenness & $1.34 \quad(.76)$ & $1.47 \quad(.98)$ & $1.20 \quad(.68)$ & $3.64 *$ \\
\hline Consumption level & $.88 \quad(2.27)$ & $1.30(3.58)$ & $.49(2.32)$ & $3.01^{\text {*t }}$ \\
\hline
\end{tabular}

Note. $S D$ in parentheses. $p<.05$ * $p<.01$ * $p<.001$. The $n$ "s for the three "partnership" categories were for males; 105, 142 and 231 and for females; 209, 101 and 163, respectively. "Responses ranged from 1 'not in the last four weeks' to 4 'daily' b Responses ranged from 1 "never" to 7 "more than 20 times" in the last 12 months. "Total number of glasses consumed in the preceding week.

\section{RESULTS}

\section{Characteristics of the Sample}

The sample consisted of 478 girls $(50 \%)$ and 480 boys. The average age was 17.4 years $(S D=7)$ at wave three. ${ }^{4}$ Approximately fifty percent of the respondent group were enrolled in secondary technical and vocational training, $24 \%$ in high schools, $1 \%$ in vocational schools, and $17 \%$ involved in another type of education. Eight percent were not enrolled in any education. The majority of the respondents ( $82 \%)$ lived with two parents, $12 \%$ with one parent, and $6 \%$ had left the parental home (e.g., lived with friend, partner, alone or with other family members). Concerning involvement in an intimate relationship, $33 \%$ of the sample $(n=314)$ indicated having a steady partner at the time of measurement. Females were more likely to have a partner $(44 \%)$ than males $(22 \%), \chi^{2}(951)=53.07, p<.001 .^{5}$ Furthermore, $30 \%$ of the males and $21 \%$ of the fermales reported having had a steady partner in the last three years but were currently nor involved. Forty-eight percent of the males and $35 \%$ of the females indicated not having a partner in the last three years. The duration of the current relationship differed between the sexes. On average, males 
had a steady partner for 13 months $(S D=10.7)$ and females for 17 months $(S D=$ $11.2), t(295)=2.96, p<.01$.

\section{Longitudinal Relations between Alcobol Use and Involvement in a Steady Relationship}

Prospective analyses were conducted to examine whether differences in drinking at the second wave were related to partnership three years later. The frequency of alcohol use and the frequency of drunkenness were positively associated with partnership at the third wave. For instance, $47 \%$ of the males with a partner reported having drunk alcohol in the last months at the second wave compared to $42 \%$ of the males who were currently not involved and $26 \%$ of the males who never had a partner (figures for females were $40 \%, 45 \%$ and $26 \%$, respectively). In addition, the level of alcohol use was positively related to partnership in both sexes. An analysis on level of consumption in which the abstainers (i.e., those not drinking in the last 6 months) were excluded, showed rather similar trends to the findings depicted in Table 5.1. Notice that the strongest differences were found between the category of subjects who had never been involved in a steady relationship and the other two categories.

The association between drinking and partnership might be curvilinear. Possibly not only abstainers (or light drinkers) but also those with high consumption levels were less likely to become involved in intimate relationships. An additional cross-tabulation was conducted in which subjects with relatively high consumption levels (90-100 percentile) were compared with the other drinking

Table 5.2. The Frequency of Drinking in Different Contexts at W/ave Two and Partnership at Wave Three

\begin{tabular}{|c|c|c|c|c|c|c|c|}
\hline \multirow{2}{*}{ Males } & \multicolumn{2}{|c|}{$\begin{array}{l}\text { Having a } \\
\text { partner }\end{array}$} & \multicolumn{2}{|c|}{$\begin{array}{c}\text { Currently } \\
\text { not involved }\end{array}$} & \multicolumn{2}{|c|}{$\begin{array}{c}\text { Never had a } \\
\text { partner }\end{array}$} & F-value \\
\hline & & & & & & & \\
\hline At home & 1.28 & $(.58)$ & 1.27 & $(.56)$ & 1.17 & $(.46)$ & $2.27 \mathrm{~ns}$ \\
\hline At a friend's place & 1.29 & $(.57)$ & 1.20 & $(.48)$ & 1.09 & $(.35)$ & 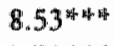 \\
\hline At parties/gatherings & 1.50 & $(.74)$ & 1.43 & $(.73)$ & 1.23 & $(.52)$ & 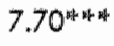 \\
\hline In PDP & 1.50 & $(.80)$ & 1.39 & $(.72)$ & 1.20 & $(.55)$ & 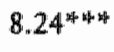 \\
\hline \multicolumn{8}{|l|}{ Females } \\
\hline At home & 1.17 & $(.46)$ & 1.19 & $(.46)$ & 1.15 & $(.39)$ & $.28 \mathrm{~ns}$ \\
\hline At a friend"s place & 1.11 & $(35)$ & 1.15 & $(.38)$ & 1.07 & $(.35)$ & $1.72 \mathrm{~ns}$ \\
\hline At parties/gatherings & 1.41 & $(.67)$ & 1.46 & (.64) & 1.22 & $(.54)$ & 6.24 \\
\hline In PDP & 1.42 & $(72)$ & 1.48 & $(.72)$ & 1.20 & $(50)$ & $7.55^{\text {称* }}$ \\
\hline
\end{tabular}

Note. $S D$ in parentheses. Responses on the frequency measures ranged from 1 "not in the last four weeks' to 4 'daily". ns non-significant, " $p<.05$. "** $p<.01$. "***t $p<.001$. PDP = public drinking places 
categories. We found no evidence of a non-linear relationship between quantity of use and partnership in both sexes.

The situation in which alcohol is used is expected to affect the association between drinking and partnership. Primarily, when people drink in settings where they have the opportunity to associate with the opposite sex (parties, pubs, discos), alcohol consumption is related to partnership. Table 5.2 shows that drinking at home was not related to partnership whereas drinking at parties and in public drinking places was positively associated with partnership three years later for both sexes.

\section{Changes in Substance Use and Intimate Relationships}

Table 5.3 displays the means of the drinking variables at the second and third wave, and the differences in level of consumption between the second and third wave for the three categories on partnership. ${ }^{6}$ It could be argued that because some people were already involved in their relationship at the second wave, they had already adjusted their drinking habits. It appeared, however, that most relationships did not endure more than 36 months (i.e., time between the last two waves). Only $3 \%$ ( $n=$ 3) of the males and $7 \%(n=19)$ of the females at wave three reported that their relationship continued for more than three years. It should be noted that only a small percentage of the subjects reduced their alcohol consumption ( $5 \%$ of the males and $7 \%$ of the females).

Young men adjusted their drinking patterns when they became involved in a steady relationship. In terms of total consumption, the strongest increase was reported by males who were not involved at the time of measurement but had experiences with steady relationships in the preceding three years. As a result, this category of adolescents had the highest consumption levels at wave three while three years earlier the category of adolescents with a partner reported the highest levels of alcohol use. More specifically, males with a partner increased their consumption in public drinking places to a lesser extent than those not dating at the time of measurement. However, this increase was comparable to that of those who did not have a partner in the preceding three years. The opposite result was found for drinking at home: the strongest increase was reported for young men with a partner.

Although the findings on levels of consumption were in most cases not significant for young women, the trends were rather similar. For instance, the lowest increase in alcohol use in public drinking places was found for those involved in a relationship. 


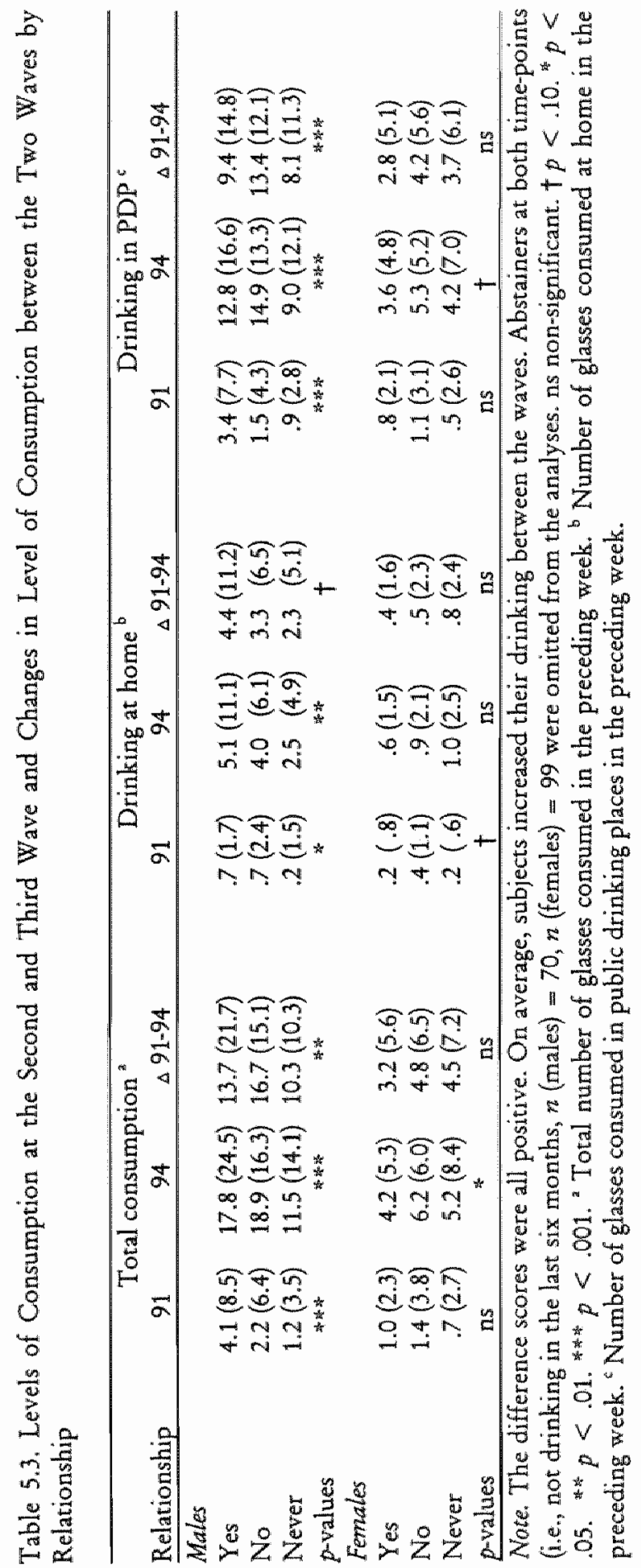




\section{Discussion}

This study investigated the associations between alcohol use and involvement in intimate relationships using data of a longitudinal study among adolescents. The results showed that drinking in middle adolescence is related to partnership in late adolescence. Those who reported higher levels of alcohol use, and higher frequency of drinking and drunkenness were more likely to be involved in a romantic relationship. Moreover, an analysis in which drinking in different contexts was related to partner ship showed that drinking in peer settings affected the likelihood of having a partner three years later while drinking at home did not. Visits to public drinking places and parties not only offer the opportunity to consume alcohol, to forget daily hassles or to enhance contacts with friends and peers (Træen \& Nordlund, 1993) but also operate as occasions on which people search for a potential partner (Sanderson \& Cantor, 1992; Silbereisen et al., 1992). ${ }^{7}$

It was hypothesized that becoming involved in a steady relationship would affect adolescents' drinking habits. According to our data, male respondents with a partner increased their alcohol levels to a lesser extent than the category of youngsters who were currently not involved. These findings can be understood in the light of the study of Silbereisen et al. (1992). The authors stated that involvement in a relationship is accompanied by changes in leisure activities - partner go to a pub or disco less often and seek each other"s company in private settings. Knibbe et al. (1987) suggested that certain roles (such as marriage, parenthood or employment) result in explicit norms and expectations about how people should behave in specific situations. Leaving the role of being single implies that people experience restrictions and social monitoring with respect to their drinking habits. Information on the opinions of people in the direct environment of youngsters (partner, friends, parents) on functioning in intimate relationships as well as data on how people spend their leisure time is required to gain more insight into the effects of partnership on drinking habits.

Why did these effects not show up in young women? An important methodological reason lies in the lower consumption levels of females. Particularly at the second wave, the average levells were rather low (ranging from .7 to 1.4 glasses per week for the three categories). Therefore, it was difficult to detect differences between the categories. It is also possible that the formation of a relationship does not affect females drinking habits. Perhaps the drinking levels are not high enough to cause a significant change. Or young men pur less pressure on their partner to change her drinking habits than the other way around. However, more in-depth research is necessary to address this issue.

The strongest increase in consumption was reported for the category of young men who have had a steady relationship but were not involved at the time of measurement. One could argue that because the previous relationship had broken down, they were drinking more intensively to reduce their feelings of stress and 
disappointment (see for research on loss of an adolescent relationship, Kaczmarek $\&$ Backlund, 1991). Although we did not find support for a negative association between heavy drinking at wave two and involvement in a relationship at wave three, the absolute levels of consumption were relatively low for the 14-15 age category. A few years later, some adolescents consume large amounts of alcohol in public drinking places and are a less attractive partner for opposite sex peers. Another explanation deals with environmental influences. Perhaps these youngsters do not experience any pressure from dating partners or friends to reduce consumption levels. They simply perceive no or less barriers to increase their drinking. Since the use of alcohol in specific settings appears to enhance the chances of partnership, they might visit public drinking places and consume alcohol more frequently. A study is required in which youngsters (and their partners) are followed intensively for a period of time (see Power \& Estaugh, 1990) to examine the merits of these explanations.

Perhaps we should focus on adolescents" dependency, satisfaction and communicational styles in relationships in order to explain changes in substance use. For instance, youngsters who are strongly attached and committed to their partner (or the relationship) experience stronger internal or external pressures to adjust their drinking habits when this is required by the partner. On the other hand, for certain categories of people, feelings of dissatisfaction within the relationship could lead to specific coping strategies, such as seeking other company in "wet" settings or drinking alone. The investment model of Caryl Rusbult (1983; Rusbult, Verette, Whitney, Slovik \& Lipkus, 1991) distinguishes relational satisfaction, investments, quality of alternatives and commitment as factors affecting accommodation processes in interpersonal relationships. It would be worthwhile to address the value of this model with respect to communication about substance use habits in adolescent relationships. In addition, adolescents' prevailing concerns in intimate relationships may moderate their likelihood of following a partner's opinions on going out and drinking. Whether they engage in a relationship with self-focused and narcissistic goals (making a positive impression on friends or becoming sexually experienced) or with more other-focused goals (with open communication and mutual dependence) might affect their vulnerability to partner influences and their willingness to change particular behaviors (Miller, Bettencourt, DeBro \& Hoffman, 1993; Sanderson \& Cantor, 1995).

Recent studies have reported that alcohol consumption and pub-going is positively related to the social development of late adolescents in terms of adequate integration in the peer group and becoming emotionally and physically more separated from the nuclear family (see Engels, Knibbe \& Drop, 1998a; Pape \& Hammer, 1996). The present study indicates that drinking in middle adolescence is positively associated with the formation of intimate relationships in late adolescence. All in all, one could argue that these youngsters are better off than those with no or limited experiences with dating and partnership. Samet and Kelly (1987), for 
instance, reported that partnership in adolescence is related to higher self-esteem and gender identity. To be more specific on this issue, data is needed on the psychological well-being of adolescents as well as on their situation in young adulthood. It is possible that some of those who experiment intensively with relationships become unhappy and frustrated in their twenties while among the "late matures", many people develop a satisfying intimate relationship later on.

Further research should reveal to what extent the presented findings on alcohol use can be extrapolated to other substances. Perhaps the use of illegal drugs, such as cocaine and heroine has a negative impact on the development of personal relations as suggested by Yamaguchi and Kandel (1985). Differences in negative side effects of substances (such as inadequate functioning in personal relationships) or the societal norms regarding these substances might account for variation in findings. Furthermore, if we follow the sample in their young adulthood (22 to 24 year-olds), heavy drinking or problem drinking in this period could have a negative effect on the formation and continuation of intimate relationships (see Newcomb $\&$ Bentler, 1988).

Our study is explorative in the sense that it does not offer information about underlying mechanisms which could be used for the construction of effective prevention programs. Nonetheless, it signifies that alcohol is part of a life style involving important values and behaviors for a large groups of adolescents. If alcohol is related to beneficial social aspects, such as integration in the peer group and development of intimate relationships, it becomes more difficult to prevent youngsters from entering alcohol related settings and consuming alcohol. Furthermore, the present study emphasizes the importance of a partner on the development of drinking habits. Empirical and theoretical studies dealing with social influence processes and substance use, however, concentrate primarily on peer and parental influences (see Conrad et al., 1992; Petraitis et al., 1995). In our opinion, attention should be paid to the relative impact (modeling and normative influences) of a partner in the total constellation of environmental influences. It is essential to know which social factors affect changes in substance use patterns in particular periods of time in adolescence especially if we consider effective prevention and intervention programs on risky behaviors.

\section{NOTES}

Based on:

Engels, R.C.M.E., \& Kribbe, R.A. (1998). Alcohol use and intimate relationships in adolescence: When love comes to town. Manuscript submitted for publication.

An earlier version of this paper is presented at the $23^{\text {rd }}$ Annual Alcohol Epidemiology Symposium of the Kettil Bruun Society for Social and Epidemiological Research on Alcohol, Reykjavik, Iceland, 2-6 June 1997. 
2" when love comes to town" is a song written by Bono (1988).

"As a result of this formulation, subjects used their own interpretation and definition of the term "steady relationship". This implies that an individual might perceive his/her informal relationship of two weeks as being steady while somebody else might perceive his/her balanced and persistent relationship of five months as being non-steady.

4 Because the data on partnership formation were exclusively gathered at the third wave, the socio-demographic characteristics of the sample referred to the third wave. This was done for the sake of clarity.

5. Two men and five women did not provide information about involvement in an intimate relationship and were excluded from further analyses.

${ }^{6}$ Those who reported abstaining (i.e., not drinking in the last six months) at both waves were excluded from these analyses. Nevertheless, it should be mentioned that the category of subjects remaining stable in their consumption included respondents who indicated not drinking alcohol in the preceding week before administration of the questionnaire at botb waves.

"One could question to what extent the positive effect of drinking on partnership is due to the social setting in which drinking takes place. However, the strong correlation between drinking wariables and the frequency of pub-going (see allso Knibbe et al., 1991) makes it difficult to draw conclusions about this issue with our type of survey data. 



\section{6}

\section{FUNCTIONS OF PUB-GOING FOR LATE ADOLESCENTS ${ }^{1}$}

Young people's alcohol use is concentrated at the weekends, and in public drinking places, such as bars, youth centers, discos, and at parties. A study in the Netherlands showed that $85 \%$ of the weekly consumption of $16-25$-year-olds took place in public drinking places (Knibbe, Oostveen \& Van de Goor, 1991). A similar concentration of juvenile drinking in situations like bars, pubs and parties is reported by researchers from other Western countries (Harford \& Grant, 1987; Thombs \& Beck, 1994; Weiss \& Moore, 1994). Going out is a significant leisure activity for youngsters in terms of time investment. Between the ages of 17 and 19, they spend about eight hours per week, mostly at the weekends, in public drinking places, organized parties and the cinema (De Zwart, Warnaar \& Van Dam, 1994).

There is a tendency in the literature to focus on the negative consequences of alcohol consumption and visits to alcohol-related settings. It is well-known that the negative side effects of drinking, such as violence, aggression, other criminal acts and traffic accidents (Hingson, Heeren, Howland \& Winter, 1991; Mayhew, Donelson, Beirness \& Simpson, 1986; Milgram, 1993) account for many problems for teenagers and their (immediate) environment. In many cases, these side effects are associated with visits to pubs and discos. However, such findings do not clarify why, despite the obvious hazards associated with pub-going, the vast majority of young people visit these places quite regularly. To gain insight into the reasons youngster have for entering alcohol-related settings, research should focus on the functions of pubgoing. 
Some researchers have dealt with the positive effects of young people's alcohol consumption (Leifman, Kühlhorn, Allebeck, Andréasson \& Romesjö, 1995; Pape $8 x$ Hammer, 1996a) and the perceived positive consequences of drinking (e.g., Christiansen \& Goldman, 1983). Less attention, however, has been paid to the beneficial aspects of the setting in which most of the alcohol is consumed. The present study examines to what extent pub-going is associated with social integration in the peer group, maturing out and psychosocial well-being.

The transition from adolescence to adulthood is accompanied by intensified contacts with peers and an entrance into new social contexts and activities. (Schulenberg et al., 1996). The concerns which adolescents have to achieve intimacy goals, such as closeness and trust, is redirected from parents towards peers. It is essential for them to come in contact with new friends or strengthen existing bonds. In this way they can reflect their own ideas, opinions and emotionall development (Johnson \& Jennison, 1992; Yamaguchi, 1990). This period also brings an increasing interest in dating and sexual experiences (Engels \& Knibbe, 1998; Samet \& Kelly, 1987). Silbereisen, Noack and Von Eye (1992) stressed that adolescents prefer settings which provide the opportunity to approach others and make progress with intimate contacts. In their study, they found that particularly discos served this purpose among the Berlin youth. Furthermore, Trzen and Nordlund (1993) showed that people's attitudes towards the social consequences of pub-going (e.g., meeting friends or establishing new relationships) affect the intentions of 16 to 24 year-olds to visit public drinking places. In addition, some studies have shown that abstinence, which is negatively associated with visiting public drinking places, is related to less likelihood of having a close friend and less time spent with their peers (Reid, 1978; Selnow \& Crano, 1988; Silbereisen \& Noack, 1988), and with low levels of sociability (Leifman et al., 1995).

At approximately the same time as youngsters seek integration in the peer group, they are inclined to distance themselves from the social control of parents and other authorities. In a longitudinal study by Jessor and Jessor (1977), young drinkers were less involved with and bonded to their parents, and experienced less influence from traditional institutions compared with abstainers. According to Jessor (1987, pp. 335), behaviors such as drinking, smoking and sexual experiences are "not necessarily irrational, perverse or pathological; for adolescents, such behaviors can fulfill important goals and can be an essential aspect of psychosocial development". Therefore, we expected pub-going to be imbedded in a framework of behaviors (e.g., having a job, spending more time with friends and less time with parents) and values (e.g., less emphasis on religion or school) which mark transitions from one life-phase into another (Kandel, 1980).

Pub-going by adolescents can also be seen as a collectively appreciated timeout situation in which it is legitimate to forget everyday obligations. It facilitates the sharing of activities, experiences and emotions with peers. The exchange of common experiences and the knowledge that others are in a similar position have a 
positive impact on youngsters" well-being (Dolcini \& Adler, 1994; Hartup, 1983). If the potential advantages of pub-going for this age group are considered, not entering these types of settings might be related to loneliness, isolation and stress. Perhaps people who do not go out regularly have less opportunities to relieve their daily stress or to associate with peers. This point of view is related to the ideas of Pape and Hammer (1996a) on alcohol abstinence in late adolescence and young adulthood. They emphasize that alcohol use is the norm for people in this period of life. Deviating from the mainstream seems to be associated with negative aspects, such as a lack of social integration as well as low self-esteem and feelings of depression.

Research on the purposes of public drinking places for adolescents may contribute to a more adequate prevention of alcohol misuse and its consequences. If, from an adolescent perspective, pub-going is more than just drinking alcohol, preventive efforts which do not take this into account are likely to have limited effects. In addition, strategies that reduce the availability of alcoholic beverages, for instance, by raising the minimum age for entering public drinking places, could have negative side effects if the setting is functional to adolescents. When particular age groups are prohibited to go to pubs and discos they might seek other settings (street, malls) which also provide a symbol of maturity or serve as an entrance to the peer group social life (Botvin et al., 1989), but in which the use of licit and illicit substances is less controllable.

The present study explores the extent to which pub-going is rellated to transitions marking the adoption of more adult behaviors, social integration and psychosocial well-being. ${ }^{2}$ Our study is explorative because we examined the correlates of pub-going at a specific moment in time. The direction of relationships could not be tested. Having a job, spending less time with parents, and developing romantic relationships were used as indicators of transition markers. Social integration included items on the number of friends, hours spent with friends, feelings of loneliness and the quality of friendships. Self-esteem and perceived stress were considered as indicators of psychosocial well-being. Data from a study of 958 late adolescents were used for analyses.

Firstly, differences in drinking behavior between adolescents who visited public drinking places, subsequently called visitors, and adolescents who did not visited public drinking places, subsequently called non-visitors, were described. It was hypothesized that visitors would report a higher frequency and quantity of alcohol use than non-visitors.

Secondly, univariate analyses were performed to investigate the relations between transition markers, social integration, psychosocial well-being, and pubgoing. We expected that visitors would score higher on other transition marking behaviors and values, would be better integrated in their nerwork of friends, and would experience feelings of distress or low self-esteem less often than non-visitors. Multivariate logistic regression analyses were performed to examine the importance of these variables in more detail. No hypotheses were formulated about which 
factors most strongly differentiated visitors from non-visitors.

In general, it was hyporhesized that pub-going would be positively associated with personal and social aspects of adolescent development. Nevertheless, it is possible that there is a curvilinear relation between pub-going and psychosocial well-being. Visiting public drinking places frequently could be associated with, for instance, problems at school or a troublesome relationship with parents. For this category of teenagers, pub-going might be interpreted as an escape from reality to forget their worries and feelings of distress. Therefore, we tested whether frequent visitors experienced more stress or low self-esteem than those who visited public drinking places less frequently. We also examined whether the two categories of visitors differed on the other independent variables.

\section{METHOD}

\section{Procedure and Sample Characteristics}

Data for this analysis were derived from a three-wave longitudinal study on predictors of smoking and drinking behavior among Dutch secondary schoolchildren. The study started in 1989 writh a sample of 1,454 students from first and second grade. Thirty-six schools differentiated by educational level and region cooperated. Twenty-nine schools refused to participate owing to privacy considerations or affiliations with other research or prevention programs. At the first measurement in the autumn of 1989, the questionnaires were administered in the classrooms. In the autumns of 1991 and 1994, forms were sent to subjects' homes. Participants who did not respond to two postal reminders were approached by phone to complete an interview. Special attention was paid to the confidentiality of responses. The letters of introduction and the questionnaires emphasized privacy aspects and clearly stated that no information about specific responses of subjects would be passed on to teachers or parents. Furthermore, matching of numbers and names was only possible by the principal researcher. The response rates were $82 \%$ at both follow-up surveys. A total of 1,063 adolescents $(73 \%)$ were enrolled in all three waves.

This paper focuses on persons in late adolescence. Therefore, data from the third measurement were used for analyses. Because the telephone interview contained a limited set of items, the sample was restricted to those who filled out the questionnaire. The sample consisted of 478 girls $(50 \%)$ and 480 boys. The average age was 17.4 years $(S D=.7)$. Approximately fifty percent of the respondents' group were enrolled in secondary technical and vocational training. Twenty-four percent of the subjects were enrolled in high schools, $1 \%$ in vocational schools, and $17 \%$ on another course. Eight percent were not enrolled in any educational establishment. The majority of the respondents $(82 \%$ ) lived with two parents, $12 \%$ with one parent, and $6 \%$ had left the parental home (i.e., lived with a friend, partner, alone or with other family members). 
To determine the nature of sample attrition, a logistic regression analysis was conducted comparing remainers (i.e., participating at all three waves) and dropouts (i.e., not participating at wave two or/and three) with data of the first measurement as predictors of drop-out. Independent variables were sex, age, level of education, church attendance, origin of the father, quantity and frequency of cigarette use and quantity and frequency of alcohol consumption. Dropouts $(n=391)$ appeared to be older, lower educated, attended a church less often and had a father of Dutch origin less often than remainers $(n=1,063)$. Small differences $(p<.10)$ were found for sex and quantity of smoking, Dropouts were more likely to be male and reported a higher number of cigarettes smoked. No differences $(p>.10)$ were found for frequency of drinking and smoking, and intensity of drinking. It must be stated that this set of variables predicted only a few percent of the attrition.

An additional attrition analysis was conducted on data of the third wave to examine whether subjects who participated in the telephone interview $(n=105)$ differed from those who filled out the questionnaire $(n=958)$. Within the set of variables sex, age, education, origin father, church attendance, smoking status, quantity of smoking frequency of drinking and $6+$ drinking, these two groups differed on education and smoking status. ${ }^{4}$ Those who did not follow any educational courses or were smokers were more likely to participate in the telephone interview. They were also more likely being male, being older and going to church less often. These differences were however small $(p<.10)$.

\section{Measures}

Visiting Public Drinking Places. Respondents were asked if they went to a pub, a dance or a disco occasionally. People who responded positively were asked how often they went to a public drinking place. Response categories ranged from (1) less than once a month to (6) more than twice a week.

Transition Markers. Six variables were indicators of the transition from adolescence to adulthood. The value put on school success was measured on a 5-point scale (1) very unimportant to (5) very important. Involvement in an intimate relationship was assessed by asking whether the respondent had a steady relationship. A no response was scored 1 and a yes 2 . A question about employment was dichotomized into answers no (1) and yes (2). In addition, subjects were asked to indicate the hours spent on education, working activities, and contacts with parents (and other family members or partner) per week. If a given question was not applicable for someone, the response was recoded to zero (hours).

Sacial Integration. Subjects were asked whether they had a same-sex close friend. A no response was recoded as 1 and a yes 2 . Changes in the number of friends was measured by asking the respondents if the number of friends they associated with 
had altered compared with the second measurement, three years ago. The response categories ranged from (1) I have fewer friends to (5) I have many more friends. T'wo items served as indicators of the perceived quality of friendships. Items were "I miss having a good friend to discuss personal issues with" and "At times, I feel that I have no one to talk to" with response categories ranging from (1) totally agree to (5) totally disagree. Thus, higher scores were associated with improved quality of friendships. Furthermore, they were asked to provide information on the hours spent with friends and on relaxing activities (e.g., watching television, listening to music and playing computer games). The latter item was used as an indicator of solitary activities.

Psychosocial Well-being. Three concepts were used to assess psychosocial well-being. A translation of the Self-Derogation Scale of Kaplan and colleagues (Kaplan et al., 1984) was used. This index is an additive sum of six items measuring a lack of selfesteem. Two examples of questions are: "I wish I could have more respect for myself" and "At times, I think I'm no good at all". Response categories were different from the original dichotomous scale and ranged from (1) totally agree to (5) totally disagree. Internal consistency was good; Cronbach's $\alpha=.78$. Higher scores implied higher levels of self-esteem. A short form of the Perceived Stress Scale (PSS; Cohen, Kamarck, \& Mermelstein, 1983) was employed to measure the degree to which the respondent's life was unpredictable, uncontrollable, or overloaded. This form is a translation of the two-factor solution examined by Hewitt, Flett \& Mosher (1992) assessing perceived general distress ( 7 items) and the perceived ability to cope with current stressors (4 items). Items were rated on a 5-point scale ranging from never to very often. The two subscales showed satisfactory internal reliability, $\alpha=.84$ and .70 , respectively, for general distress and ability to cope. The items of the perceived-ability-to-cope scale were recoded. Thus, higher scores were associated with increased stress on both scales.

Alcobol Consumption. The question: "Have you drunk alcohol in the last six months?" was used to determine abstaining from alcohol consumption. Subjects who had consumed alcohol in the preceding four weeks were asked the number of glasses they had drunk during weekdays and the weekend of the preceding week. A distinction was made between the consumption outside and inside their (parental) home. The responses on these items which covered the weekly consumption were summed to serve as a measurement of the quantity of alcohol use. Both questions on the frequency of $6+$ drinking and frequency of drunkenness in the preceding 12 months ranged from (1) never to ( 7 ) more than twice a week.

\section{Data Analyses}

First, we examined the differences in scores on indicators of drinking between subjects who visited drinking places and subjects who did not visit public drinking places. T-tests and contingency tables with chi-square statistics were used for the 
variables abstinence, level of weekly consumption, frequency of drinking, frequency of $6+$ drinking and frequency of drunkenness. Abstainers were omitted for the analyses on the last four drinking measures. T'o test whether pub-going was associated with transition markers, social integration and psychosocial well-being, twotailed $t$-tests were performed. Since this study aims to explore the diversity of aspects associated with pub-going, we did not use a sum score of the indicators of the three "groups" of variables. A logistic regression analysis was used to examine the associations of pub-going multivariately. Variables were included in analyses by a forward selection procedure and the likelihood ratio criterion was used to determine which variables should be added to the model. To examine whether intensive pub-going (i.e., twice or more times a week) might be negatively associated to, for instance, social interaction or psychosocial well-being, $t$-tests were performed in which frequent visitors were compared with those subjects who visited public drinking places less frequently (i.e., once a week or less often).

Table 6.1. Associations between Alcohol Measures and Visiting Public Drinking Places

\begin{tabular}{|c|c|c|c|}
\hline & $\begin{array}{c}\text { Non-visitors } \\
(n=110)\end{array}$ & $\begin{array}{l}\text { Visitors } \\
(n=831)\end{array}$ & povalues \\
\hline Abstainung $(\%)^{2}$ & 31.5 & 6.8 & 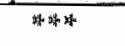 \\
\hline Drinking $>2$ days a reek ${ }^{b}$ & 2.6 & 9.3 & 하하나 \\
\hline $6+$ drinking once a week & 1.3 & 21.2 & $x+4$ \\
\hline Drunkenness once a wreek & 0 & 5.4 & 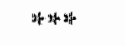 \\
\hline Weekly consumption" & $1.5 \quad(3.3)$ & $10.1(14.6)$ & 幽此- 此 \\
\hline
\end{tabular}

Note. $P$-values are based on two-tailed $t$-tests and chi-quare tests. ${ }^{*+34} p<.001 .{ }^{2}$ Abstainers are those not reported alcohol use in the past six months. ${ }^{b}$ Abstainers were excluded for the analyses on the four drinking variables. "Number of glasses consumed in the last week. $S D$ in parentheses.

\section{RESULTS}

\section{Visiting Public Drinking Places and Alcobol Consumption}

Most of the respondents reported going to a public drinking place once in a while. Only $54(12 \%)$ women and $56(12 \%)$ men did not visit a pub or disco occasionally. The findings clearly demonstrated that visits to pubs and discos is positively related to the level of weekly consumption as well as to the frequency of drinking (Table 6.1). Pub-going is also associated with indicators of heavy drinking, such as the frequency of $6+$ drinking and frequency of drunkenness. Since it is well known that boys and girls differ in their drinking patterns, the analyses were also performed by sex. Although, on average, men scored higher on every alcohol measure than women, the direction of the findings were comparable to those for the total sample. 
Table 6.2. Comparisons of Scores on Transition Markers, Siocial Integration and Psychosocial Well-being between Visitors and Non-visitors of Public Drinking Places

\begin{tabular}{|c|c|c|c|c|c|}
\hline \multirow[b]{2}{*}{ Transition markers } & \multicolumn{2}{|c|}{$\begin{array}{c}\text { Non-wisitors } \\
(n-10)\end{array}$} & \multicolumn{2}{|c|}{$\begin{array}{c}\text { Visitors } \\
(n=831)\end{array}$} & \multirow[t]{2}{*}{ two tailed $t$-test } \\
\hline & & & & & \\
\hline Value on education & 4.31 & (.82) & 4.06 & $(.90)$ & $2.73^{4+4}$ \\
\hline Hours on education & 23.84 & $(19.25)$ & 24.73 & $(17.35)$ & $.50 \mathrm{~ns}$ \\
\hline Intimate relationship & 1.15 & $(.35)$ & 1.35 & $(48)$ & $4.41^{3+24}$ \\
\hline Work & 1.30 & $(.46)$ & 1.42 & $(.49)$ & 2.58 *⿻一𠃋十 \\
\hline Hours on work & 7.06 & $(13.52)$ & 10.37 & $(13.31)$ & $2.45^{* 2}$ \\
\hline Hours with parents & 19.67 & $(21.54)$ & 19.16 & $(18.06)$ & $.27 \mathrm{~ns}$ \\
\hline \multicolumn{6}{|l|}{ Social integration } \\
\hline Best friend & 1.89 & $(.31)$ & 1.97 & $(.17)$ & 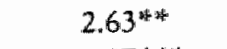 \\
\hline Number of friends & 3.35 & $(90)$ & 3.75 & $(.84)$ & 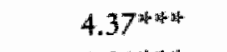 \\
\hline Discussing personal issues & 3.59 & $(1.53)$ & 4.12 & $(1.31)$ & 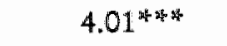 \\
\hline No feelings of loneliness & 3.86 & $(1.42)$ & 4.25 & $(1.20)$ & $2.82^{254}$ \\
\hline Hours with friends & 10.98 & $(12.52)$ & 13.75 & $(12.45)$ & $2.20^{*}$ \\
\hline Hours alone & 17.91 & $(14.22)$ & 15.40 & $(10.71)$ & $1.79+$ \\
\hline \multicolumn{6}{|l|}{ Psychosocial well-being } \\
\hline General distiress & 2.40 & $(.73)$ & 2.40 & $(.69)$ & $.03 \mathrm{~ns}$ \\
\hline Inability to cope & 2.39 & $(.65)$ & 2.34 & $(.61)$ & $.78 \mathrm{~ns}$ \\
\hline Self-esteem & 3.91 & $(.83)$ & 3.91 & $(80)$ & $.03 \mathrm{~ns}$ \\
\hline
\end{tabular}

Note. $S D$ in parentheses. ns nonsignificant. $+p<.1{ }^{*} p<.05 .{ }^{* 4} p<.01 .{ }^{* 4 *} p<.001$.

Univariate Analyses on Transition Markers, Social Integration and Psychosocial Wellbeing

T-tests were conducted to examine the scores on the 15 independent variables berween visitors and non-visitors (Table 6.2). Significant differences between nonvisitors and visitors were apparent on the item involvement in an intimate relationship. Non-visitors were less likely to have a steady partner than visitors. Furthermore, non-visitors attached more value to their educational aspirations, had a paid job less often and spent less hours on working activities than visitors. Having a best friend was more common among people who visited public drinking places. Visitors were more likely to acquire more friends over time than were adolescents who never went to a public drinking place. In addition, non-visitors indicated missing a friend to discuss personal issues with more often and reported feelings of loneliness more often. Small differences in the hypothesized direction were found for hours spend with friends and hours spend alone. No significant differences were found on levells of general distress, inability to cope with stressors and self-esteem between visitors and non-visitors. 


\section{Logistic Regression Analyses of Model Variables on Pub-going}

A logistic regression analysis was performed to examine the joint effects of the predictor variables on pub-going. A general model was tested which included the 15 model variables (Table 6.3). Attaching less value to educational aspirations, having a steady partner, spending hours on work were associated with visiting public drinking places. In addition, having a best friend, the number of friends, and the hours spend with friends were positively related to pub-going. Having feelings of loneliness and hours spending alone were negatively related to pub-going.

To examine possible effects of gender, we tested a second model in which the variables were included in the first step and the interaction terms of the 15 model variables with sex in the second step. It appeared that no interaction terms were included in the equation after the eight model variables were included.

Table 6.3. Logistic Regression of Model Variables on Visiting Public Drinking Places

\begin{tabular}{lccc}
\hline & OR & $\begin{array}{c}\text { Regression } \\
\text { Coefficient }\end{array}$ & $\begin{array}{c}95 \% \\
\mathrm{CI}\end{array}$ \\
\hline Value on education & .62 & $-.47^{* 4}$ & $.46-.84$ \\
Intimate relationship & 3.01 & $1.10^{* * * *}$ & $1.68-5.39$ \\
Work & 1.77 & $.57^{*}$ & $1.09-2.86$ \\
Best friend & 3.21 & $1.17^{* *}$ & $1.33-7.73$ \\
Number of friends & 1.63 & $.49^{* 4 *}$ & $1.25-2.13$ \\
No feelings of loneliness & 1.25 & $.22^{4 *}$ & $1.06-1.47$ \\
Hours with friends & 1.02 & $.02^{*}$ & $1.00-1.05$ \\
Hours alone & .97 & $-.02^{* 4}$ & $.96-.99$ \\
\hline
\end{tabular}

Note. The odds ratios presented in this table are adjusted for the other variables in the model. * $p<.05$. * $p<.01$.*** $p<.001$.

\section{Are There Negative Effects of Frequent Pub-going?}

Frequent visits to public drinking places might be interpreted as a way of coping with problems and negative feelings. Therefore, we compared those who visited public drinking places twice or more times a week with those who visited these places once a week or less often. Table 6.4 shows the significant differences in scores on the model variables for the two categories. Adolescents who visited pubs and discos frequently spent less time on their education but spent more time on their work and with friends. Furthermore, they acquired more friends over time. Frequent visitors less often had a steady relationship. In general, there was no indication that this category experienced stronger feelings of stress or low selfesteem, or were less integrated in their peer group. 
Table 6.4. Comparisons of Scores of Transition Markers, Social Integration and Psychosocial Well-being between Visitors and Frequent Visitors

\begin{tabular}{|c|c|c|c|c|c|}
\hline \multirow[b]{2}{*}{ Hours on education } & \multicolumn{2}{|c|}{$\begin{array}{c}\text { Visitors } \\
(n-614)\end{array}$} & \multicolumn{2}{|c|}{$\begin{array}{l}\text { Frequent visitors } \\
(n=224)\end{array}$} & \multirow{2}{*}{$\frac{\text { two tailed t-test }}{3.73^{\text {th }}}$} \\
\hline & 28.18 & $(17.30)$ & 21.07 & $(17.05)$ & \\
\hline Hours on work & 9.82 & $(12.69)$ & 12.80 & $(14.91)$ & $2.62^{4 *}$ \\
\hline Intimate relationship & 1.37 & $(.48)$ & 1.30 & $(46)$ & $1.75+$ \\
\hline Number of friends & 3.70 & $(.85)$ & 3.98 & $(.72)$ & 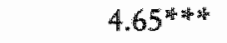 \\
\hline Hours with friends & 12.49 & $(11.37)$ & 16.54 & $(13.06)$ & $4.28 * 4$ \\
\hline
\end{tabular}

Note. SD in parentheses. Frequent visitors went to public drinking places twice or more times a week. Visitors went to public drinking places once a week or less often. Only the significant differences $(p<.10)$ are presented. $+p<.1 . * 0.05 .{ }^{*} p<.01 . * *^{*} p<.001$.

\section{DISCUSSION}

Pubs and discas are settings in which young people come together and consume alcohol. In this paper, we looked for other functions of public drinking places for youngsters in the Netherlands. First, pub-going was associated with stronger social integration in peer networks. Those who visited public drinking places were more likely to have a close friend, acquired more friends over time, spent more time with their friends and spent less time alone compared to non-visitors. Additionally, nonvisitors judged the quality of their relationships with others differently from visitors: they were more likely to miss having a friend to discuss personal issues with. They were also less likely to have feelings of loneliness. In other words, visiting public drinking places is indicative of a way of life which facilitates social integration (see Feinhandler, 1986, in Johnson \& Jennison, 1992).

Love and romance are one of the prevailing concerns of adolescents (Berndt, 1982; Paul 8x White, 1990). Research has shown that establishing a romantic relationship and having their first sexual experiences is important for their emotional development and intimacy needs (Sanderson \& Cantor, 1995). Our results support the study of Silbereisen et al. (1992) which indicating that public drinking places serve the opportunity to meet the opposite sex and to start a romantic relationship - the multivariate analysis clearly showed that visitors were more likely to be involved in a steady relationship than non-visitors.

The process of gaining autonomy and detachment from parents, which are important normative transitions for late adolescents (see Moore, 1987), was not investigated in our study. Nevertheless, there are some indirect suggestions that pub-going is related to this separation process. One of the aspects of the psychological separation process deals with emotional independence or detachment (Hoffman, 1984). Since visitors have more opportunities to get support from peers or a partner they might consider it easier to become independent from their parents. In addition, earning money, which is more common among visitors, is also considered to be one 
of the features of independence. Therefore, we assume that further research providing detailed information about the parent-adolescent relationship will show that pub-going is positively associated with this separation process.

No differences were found between the indicators of psychological well-being and visiting public drinking places. This might be due to the age group used in the current study. It is possible that in young adulthood, when most persons visit pubs for several years, continued absence from settings with evident social functions will lead to feelings of dissatisfaction and to a decrease in self-confidence (see Pape $8 x$ Hammer, 1996a). Another explanation deals with the assessment of self-esteem. Brown and Alexander (1991) used a scale covering four areas namely family acceptance, academic competence, peer popularity and personal security. If a more specific scale had been used in our study, it might have provided more in-depth information on how people feel and behave in peer groups. A third explanation has to do with selective association (Bauman \& Ennett, 1996; Engels, Knibbe, Drop \& De Haan, 1997). Some youngsters who do not visit pubs and discos (or use alcohol) might undertake activities (e.g., sports, attending church, playing music) in which they meet peers with similar preferences and habits. Functioning in these networks may not be related to low self-esteem or distress for at least certain categories of youngsters. A similar point is raised by Pittman (1995) who stressed the relevance of norms in subpopulations. Abstinence and not visiting public drinking places could be features of a lifestyle which are labeled as positive in some sub-cultures. For instance, certain religious movements are prominent in some villages in the Netherlands and have regulations which do not allow alcohol consumption or exposure to alcohol-related situations. Although the size of our sample did not permit a further differentiation it would be worthwhile to investigate the heterogeneity of the groups of non-visitors and abstainers.

Elliott, Huizinga and Ageton (1985) suggested that deviating from a relatively normal psychosocial development would result in high levels of substance use. Although our study focused on the category of non-visitors as "deviant" in late adolescence, frequent pub-going could also be seen as deviating from the mainstream. For instance, youngsters who play truancy because of problems at school or in the family could seek settings in which parents and teachers have low levels of control and power over them. Within our study, however, we found no evidence for negative effects of frequent pub-going. It must be said that no data was available on possible causes of poor psychological status. Some researchers reported that poor academic grades (Bailey \& Hubbard, 1990) and having divorced parents (Baumrind, 1985) were indicative of drug use and perhaps also of frequent pub-going.

A limitation of the current study is that we could not carry out a longitudinal investigation. None of the social integration and psychosocial well-being variables were measured at earlier waves. Therefore, this study could not examine the long term effects of a limited exposition to certain situations but displayed the correlates of pub-going at a specific time-point. This affects the generalizability of our results. 
For instance, those who do not visit pubs and discos might be late maturers. Thus, some of the differences in social and emotional development could disappear if our sample were reinterviewed in their mid-twenties. On the contrary, some adolescents who do not experience what for the majority of youngsters are important changes in late adolescence might encounter problems with the detachment from the nuclear family or the formation of intense friendships in young adulthood.

Since the main result of this study could be viewed as encouraging visiting alcohol-related settings it should be stressed that the main goal was to explore pubgoing from a different perspective. We acknowledge the negative effects of adolescent drinking in terms of violence, aggression or drunk driving, and stress that some drinking patterns in adolescence could lead to problem drinking or binge drinking in young adulthood (e.g., Pape \& Hammer, 1996b; Schulenberg et al., 1996). Gathering data on facilitating effects as well as negative outcomes of youngsters' drinking patterns would benefit future studies.

It is questionable to what extent our findings can be generalized to other countries and cultures. The legal age for entering public drinking places in the Netherlands is 16 years. At this age, youngsters are allowed to order soft alcoholic beverages, such as beer and wine. Since in some western countries the legal age for pub-going or alcohol use happens to be higher, attention must be paid to the significance of pub-going across age groups and comparability of findings across countries. The importance of religion is another issue which deserves attention. Religious affiliation in terms of frequent church attendance or strong religious values has been regarded as a sign of late maturation (Donovan \& Jessor, 1985; Jessor \& Jessor, 1977). In the Netherlands where most adolescents do not attend a church or religious gathering on a regular basis (SCP, 1994), the absence of church visits can not be understood in terms of transition marking.

Although our findings on the beneficial effects of pub-going are considered to be preliminary, we wish to raise a few points of concern for the prevention of alcohol misuse and its consequences. First, the vast majority of late adolescents visit pubs and discos in the Netherlands. This large proportion implies that pub-going is not only a socially acceptable behavior but also normative. Therefore, health education which aims to discourage people visiting these settings are expected to have limited effects.

Further legislation on adolescent pub-going, for example, raising the minimum age for accessing public drinking places could have some negative consequences. Young people will still seek opportunities to meet each other and might end up in places (in the street, shopping centers, at a friend's home without the company of adults) in which alcohol consumption is less controllable than in pubs and discos. Although it might sound paradoxical, an advantage of the drinking habits of adolescents remains in the fact that they are concentrated in specific public settings. One strategy to reduce levels of alcohol use could exist in changing aspects of the drinking situation while maintaining the purpose of the setting. Observational 
studies suggest that variations in alcohol consumption partly depend on the tempo of the music (Bach \& Schaefer, 1979), the seating arrangements (Cutler \& Storm, 1975) and the sex-ratio of the visitors (Knibbe, Van de Goor \& Drop, 1993). In addition, creating opportunities to play games or to dance might affect overall consumption levels. Policies which, in cooperation with the local authorities and owners of bars and discos, try to focus on contextual characteristics of the drinking setting have potential value.

Young people might still have different reasons for visiting public drinking places. Perhaps the majority of youngsters visit pubs and discos, and subsequently drink alcohol, for social reasons (e.g., Christiansen \& Goldman, 1983). They might be in control of their drinking habits and might not suffer from any acute or chronic negative effects. Those who judge drinking places as primarily related to alcohol, and who also have robust and positive beliefs about the effects of alcohol, could be the ones with high consumption patterns and alcohol-related problems later on. In terms of social consequences, a point of concern is the number of youngsters who become involved in fights and violence after the use of alcohol in pubs and discos. Police surveillance and efficient transportation (busses, taxis) to and from the scene might help to prevent criminal acts (see Leegwater, 1996). With respects to these negative effects, we assume that health education interventions which concentrate on risk-prone categories of youngsters are more effective in the prevention of certain side effects than school-based programs which concentrate on youngsters in general.

This also leads to the question of which parties should be included in programs targeting people in their late adolescence. Since the period between adolescence and young adulthood is marked by feelings of independence, disentanglement of family ties and lower levels of parental control, parents may not be the most appropriate party to guide youngsters into "safe" drinking levels. Incorporating parents in a prevention program (see for examples Perry et al., 1993; Klitzner, Greunewald \& Bamberger, 1990) might even have a boomerang effect.

\section{NOTES}

\section{"Based on:}

Engels, R.C.M.E., Knibbe, R.A., \& Drop, M.J. (1998). Visiting public drínking places: An explorative study into the functions of pub-going for late adolescents. Accepted for publication in Substance Use and Misuse.

Engels, R.C.M.E., \& Knibbe, R.A. (1997). De zonnige zijde van alcoholgebruik. De betekenis van drinken en uitgaan voor jongeren. Tijdschrift voor Alcobol, Drugs en andere Psychotrope Stoffen, 22, 154-163.

An earlier version of this paper is presented at the 22, Annual Alcohol Epidemiology Symposium of the Kettil Bruun Society, Edinburgh, Scotland, 1996.

2 The three perspectives on pub-going presented in this paper are not entirely exclusive. For 
instance, an adequate integration in a peer netwrork is associated with behaviors and values marking the transition from adolescence to young adulthood. In essence, it aims to structure the literature and thoughts on this topic.

"The fact that we present analyses on cross-sectional data of a longitudinal study might seem awkwrard. Because almost all independent variables were exclusively included in the third wave, we could not test our hypotheses prospectively.

4 The sets of predictor variables in the two attrition analyses were not similar because the questionnaire for the third wave was adjusted for the raising age of the respondents. 


\section{WHY DO LATE ADOLESCENTS DRINK AT HOME? A STUDY ON PSYCHOLOGICAL WELL-BEING, SOCIAL INTEGRATION AND DRINKING CONTEXT ${ }^{1}$}

In the course of adolescence, alcohol use becomes common for the majority of people. The high prevalence of drinking in late adolescence indicates that drinking is a socially acceptable behavior (e.g", Johnston, O'Malley \& Bachman, 1994; Mann, Chassin \&x Sher, 1987). According to Jessor (1991), drinking is one of the behaviors which might have beneficial features for youngsters" psychosocial development. Nonetheless, alcohol use might serve other functions. For instance, drinking is often interpreted as a coping mechanism to alleviate feelings of anxiety, depression, stress or low self-esteem (Conger, 1956). When youngsters adopt the idea that a solution to their problems and distress can be found in a bottle, they could be at risk for alcohol problems as adults. The present study discusses stress-reducing and social aspects of drinking from the perspective of the setting in which young people consume alcohol.

It is widely believed that some people drink in response to stress (Cooper, Russell, Skinner \& Windle, 1992). It gives them some short-term relief from the unpleasant effects of strain and conflict. The underlying causes of emotional distress among adolescents are diverse. Elliott, Huizinga and Ageton (1985) stated that adolescents with a poor attachment to the nuclear family, or a lack of commitment to school and academic achievement, are more prone to seek out deviant peers and to initiate drinking. In particular, low academic achievement and a problematic familly environment (e.g., divorced parents, living in deprived areas) are factors leading to stress and, eventually, to the initiation of alcohol use or to heavy drinking (Kumpfer $8 x$ Turner, 1990). The rationale behind the pathways from low 
self-esteem to alcohol use are quite comparable to those proposed for stress. According to Kaplan and co-workers (1984), feelings of rejection or being unwanted by 'conventional others' result in withdrawal from traditional values, to $\mathrm{a}$ search for unconventional company, and to the taking up of deviant behavior. In this scenario, self-esteem will be enhanced when youngsters can share their values and behaviors in a new group of 'similar' others.

Empirical results are mixed about the direct relationships between indicators of psychosocial well-being and substance use. Whille some studies have reported that low self-esteem and high levels of stress are related to heavy drinking or problem drinking among young people, others have failed to demonstrate significant relationships (see review by Petraitis, Flay and Miller, 1995). An explanation for this ambivalence is that the relationships are much more complex. In a study on midadolescents, Windle and Windle (1996) stressed that personal and social factors affect the relationship between stressors and problematic behaviors. For instance, research on general styles of coping (Endler \& Parker, 1994; Folkman \& Lazarus, 1988; Moos et al., 1982) and alcohol-specific coping styles (Cooper et al., 1992) has shown that, in contrast to problem-focused behaviors, emotional-focused coping or avoidance strategies are rellated to alcohol misuse and heavy drinking, especially under conditions of stress. Another line of inquiry directs attention to social support as a mediating factor (e.g., Pierce, Sarason \& Sarason, 1992). The general hypothesis is, that whether stress leads to deviant behavior depends on the amount of social support. For example, when someone is not performing well at school and might become a drop-out, the amount of support s/he will get from parents and friends will affect whether problem behaviors come into sight and are attractive solutions for stress and negative feelings experienced.

The notion of social support in the stress-alcohol relationship directs attention to the context of drinking. Although most youngsters start to drink in the company of parents, over the years they drink more often in the surroundings of peers and outside the parental home. In the Netherlands, about $80 \%$ of young people's total consumption is concentrated in public drinking places and at parties (Knibbe et al., 1993). This pattern of young people drinking primarily outside the parental home is also apparent in other Western countries (Harford and Grant, 1987; Weiss \& Moore, 1994; Wilks and Callan, 1987). According to Engels, Knibbe and Drop (1998a), visiting public drinking places is associated with an adequate integration in peer networks and with sociability. These latter findings coincide with recent research on facilitating aspects of drinking, stating that moderate drinking is a normative behavior for the well-adjusted, socially integrated and assertive young adult (e.g., Leifman et al., 1995; Pape, 1997; Winefield et al., 1989). Concluding, the social functions of drinking in peer settings might affect the eventual association between distress and alcohol use. In particular, drinking in nonpeer settings, like drinking solitarily, or with parents, at home, might be associated with distress. 


\section{The Present Study}

Two approaches are employed in this study to explore the relationships between distress, alcohol and context. Cross-sectional data from a study among 17 to 19 yearolds in the Netherlands were used for analyses. In the first approach, it is postulated that drinking levels in public drinking places and at parties are not associated with distress whereas drinking levels at home are positively associated with distress (i.e. higher consumption levels are related to low self-esteem and high levels of stress).

A small percentage of youngsters drink exclusively at home and subsequently refrain from positive social effects of drinking. They might have several reasons for not going to (and drinking in) bars and discos. For example, they might be less sociable and have no friends to go out with. Another interpretation is that youngsters who are rejected by others and have dropped out of the peer group become loners, and drink out of frustration and dissatisfaction. Thus it is possible that only for a selection of youngsters, drinking is related to distress (second approach). To explore this possibility, drinking levels were related to stress and selfesteem in three categories, namely, youngsters who drink exclusively at home, those who drink exclusively in public drinking places and those who drink in both settings. In an additional analysis, the interpretation of a possible socially deprived situation of 'home drinkers' was explored by comparing the three categories on indicators of social integration.

\section{METHOD}

\section{Procedure and Sample Characteristics}

Data for this paper were derived from a three-wave longitudinal study on predictors of smoking and drinking behavior among secondary schoolchildren in the Netherlands (Van Reek, Knibbe \& Van Iwaarden, 1993). In 1989, the study started with a sample of 1,454 students from first and second grade. Thirty-six schools differentiated by educational level and region cooperated. For the first measurement, the questionnaires were administered in the classrooms. In 1991 and 1994, forms were sent to participants" homes. Participants who did not respond to two postal reminders were approached by phone to complete an interview. The response rates were $82 \%$ at both follow-up surveys. A total of 1,063 adolescents $(73 \%)$ were enrolled in all three waves.

This paper focuses on persons in late adolescence only. Data from the third measurement were used for analyses. Because the telephone interview contained a limited set of items, the sample was restricted to those who filled out the questionnaire. The sample consisted of 478 girls (50\%) and 480 boys whose average age was 17.4 years $(S D=.7)$. Approximately fifty percent of the respondents were enrolled in secondary technical and vocational training, twenty-four percent in high 
schools, $1 \%$ in vocational schools and $17 \%$ other courses. Eight percent were not enrolled in any educational establishment. Of this latter category, $74 \%$ had a paid job. The majority of the respondents $(82 \%)$ lived with two parents, $12 \%$ with one parent and $6 \%$ had left the parental home.

Logistic regression analyses were conducted to verify whether there were differences between respondents and drop-outs (see chapter 4). No significant differences $(p>10)$ were found in relevant behavioral measures, such as the frequency and quantity of alcohol consumption.

\section{Measures}

Alcobol Consumption. Subjects who had consumed alcohol in the preceding four weeks were asked the number of glasses they had drunk during the previous week. A distinction was made between the consumption at the (parental) home and in public drinking places or at parties. The responses on these items which covered the weekly consumption were summed to serve as a measurement of the quantity of alcohol use. In this paper, abstaining is defined as not drinking alcohol in the past week.

Psycbological Well-being. Three concepts were used to define well-being. A translation of the Self-Derogation Scale of Kaplan and colleagues (Kaplan et all, 1984) was used. This index consists of six items measuring lack of self-esteem. Two examples of items are: "I wish I could have more respect for myself" and "At times, I think I'm no good at all". Response categories were adjusted from the original dichotomous scale to a five-point scale (1) totally agree to (5) totally disagree. Cronbach's alpha was .78. Higher scores mean lower levels of self-esteem. A short form of the Perceived Stress Scale (PSS; Cohen, Kamarck \& Mermelstein, 1983) was employed to measure the degree to which the respondent perceived his/her life was unpredictable, uncontrollable, or overloaded. This form is a translation of a twofactor solution (Hewitt, Flett and Mosher, 1992) that assesses perceived general distress ( 7 items) and the perceived ability to cope with current stressors (4 items). Items were rated on a 5-point scale ranging from (1) never to (5) very often. The two subscales 'general distress" and 'ability to cope' showed satisfactory internal reliability, $\alpha=84$ and 70 , respectively. The items of the perceived-ability-to-cope scalle were recoded. Thus, higher scores were associated with increased stress on both scales. The sumscore of the three scales was used as an indicator of psychological well-being $(\alpha=73)$.

Social Integration. Subjects were asked whether they had a same-sex close friend (no $=1$, yes $=2$ ). Changes in the number of friends were measured by asking the respondents if the number of friends they associated with had altered compared to the second measurement, three years before. The response categories were defined as (1) I have fewer friends or the same number of friends and (2) I have more friends. Involvement in an intimate relationship was assessed by asking whether the 
respondents (have) had a steady relationship $($ no $=1$, yes $=2)$. Two items served as indicators of the perceived quality of friendships. These items were: "I miss having a good friend to discuss personal issues with" and "At times, I feel that I have no one to talk to" with response categories ranging from (1) totally agree to (5) totally disagree. Scores on both questions were coded as (1) I can not discuss personal issues II have feelings of loneliness and (2) I can discuss personal issues / I have no feelings of loneliness. The respondents were also asked to provide information on the hours spent with friends ( 1 ' $\leqslant 8$ hours per week', 2 '> 8 hours per week'). Thus, higher scores are associated with higher social integration for all indicators.

\section{Data Analyses}

Descriptive information is provided for the scores on the indicators of psychological well-being and the alcohol consumption measures. T-tests were used to examine the differences between males and fernales. Abstainers were omitted from all analyses. Finally, two strategies were employed to examine the associations between drinking and well-being. First, Pearson correlations were calculated for all drinkers to examine the relationship between the levels of use at home and in public drinking places on the one hand, and stress and self-esteem on the other. The scores on the alcohol measures were log transformed.

Second, correlations were calculated between drinking and stress and selfesteem in the three categories of drinkers (i.e., 'home drinkers', 'public drinkers' and 'drinkers in both settings"). Furthermore, we examined whether those who drink exclusively at home might differ from those who drink in public drinking places (or in both settings) on several indicators of social integration. ${ }^{2}$ Contingency tables with chi-square statistics were used to look into differences between categories of drinkers.

Table 7.1. Means and Standard Deviations of Stress, Self-esteem and Alcohol Use by Gender

\begin{tabular}{lrrrrr}
\hline & \multicolumn{2}{c}{ Males } & \multicolumn{2}{c}{ Females } & \multicolumn{1}{c}{ Sign } \\
& M & SD & M & SD & t-test \\
\hline General distress (1-5) & 2.22 & .64 & 2.61 & .68 & $p<.001$ \\
Inability to cope (1-5) & 2.30 & .65 & 2.39 & .54 & $p<.05$ \\
Lack of self-esteem (1-5) & 1.94 & .75 & 2.26 & .82 & $p<.001$ \\
Psych. well-being (1-15) & 6.46 & 1.61 & 7.24 & 1.64 & $p<.001$ \\
& & & & & \\
Weekly consumption & 17.90 & 17.95 & 6.67 & 6.83 & $p<.001$ \\
Alcohol at home & 4.17 & 7.46 & 1.05 & 2.25 & $p<.001$ \\
Alcohol in PDP & 13.73 & 13.92 & 5.62 & 5.94 & $p<.001$ \\
\hline
\end{tabular}

Note. PDP = public drinking places 


\section{RESULTS}

\section{Descriptive Data on Well-being and Alcobol Medsures}

Table 7.1 depicts the scores on stress, self-esteem and levels of alcohol use for boys and girls. The findings showed that boys experienced less general distress, were better able to cope with stressors and reported fewer feelings of low self-esteem than girls. Significant gender differences were displayed for the alcohol measures. Boys drank about three times more glasses per week at home and in public drinking places than girls.

\section{Correlations between Well-being Variables and Drinking}

Table 7.2 displays the correlations between the scores on indicators of well-being and alcohol consumption by gender. First, the analyses for male drinkers displayed no significant associations ( 0 out of 8 ) between stress, self-esteem and alcohol use. For girls, the findings were somewhat different. The analyses showed that levels of alcohol use in public drinking places were negatively associated with low self-esteem and general stress - those who drank more glasses per week indicated fewer feelings of stress and low self-esteem. For drinking at home, no significant relationships were found.

Table 7.2. Correlations between Self-esteem, Perceived Stress and Levels of Alcohol Consumption for the Total Sample by Gender.

\begin{tabular}{|c|c|c|c|c|}
\hline Level of consumption & $\begin{array}{c}\text { General } \\
\text { stress }\end{array}$ & $\begin{array}{c}\text { Inability to } \\
\text { cope }\end{array}$ & $\begin{array}{l}\text { Lack of } \\
\text { self-esteem }\end{array}$ & $\begin{array}{l}\text { Psychological } \\
\text { well-being }\end{array}$ \\
\hline \multicolumn{5}{|l|}{ Males $(n=323)$} \\
\hline in public drinking places & .02 & .10 & .05 & .07 \\
\hline $\begin{array}{l}\text { at home } \\
\text { Females }(n=276)\end{array}$ & .01 & .10 & .04 & .06 \\
\hline in public drinking places & $-.13^{\text {故 }}$ & .09 & $-19^{-14}$ & $-.18^{*+4}$ \\
\hline at home & .03 & -.02 & -.01 & .01 \\
\hline
\end{tabular}

Note. ${ }^{*} p<.05,{ }^{* * 4} p<.01,{ }^{* * 4} p<.001$. No asterisk implies that the association was nonsignificant $(p \geq 10)$.

Table 7.3 depicts the correlations by drinking category. For boys, these analyses demonstrated that only in the category of 'home drinkers", were levels of alcohol consumption positively related to general distress, inability to cope with stressors and low self-esteem. ${ }^{3}$ In none of the examined relationships in the other two categories were scores on stress and self-esteem (positively or negatively) related to levels of alcohol consumption. For girls, the analyses by drinking category 
displayed that, especially among those who drink in both settings, drinking levels in public drinking places were associated with a higher psychological well-being. In the categories of 'home drinkers" and 'public drinkers', no significant associations were found between the indicators of well-being and levels of alcohol use.

Table 7.3. Correlations between Self-esteem, Perceived Stress and Levels of Alcohol Consumption by Drinking Category and Gender

\begin{tabular}{|c|c|c|c|c|}
\hline Level of consumption & $\begin{array}{c}\text { General } \\
\text { stress }\end{array}$ & $\begin{array}{c}\text { Inability to } \\
\text { cope }\end{array}$ & $\begin{array}{c}\text { Lack of } \\
\text { self-esteem }\end{array}$ & $\begin{array}{l}\text { Psychological } \\
\text { well-being }\end{array}$ \\
\hline \multicolumn{5}{|l|}{ Males } \\
\hline Home drinkers $(n=70)$ & $.25^{*}$ & $.29 *$ & $.32^{3 * 4}$ & $.32^{2+4}$ \\
\hline Public drinkers $(n=130)$ & .02 & .10 & .11 & .10 \\
\hline \multicolumn{5}{|l|}{ Both settings $(n=123)$} \\
\hline at home & -.01 & .10 & .05 & .06 \\
\hline $\begin{array}{l}\text { in PDP } \\
\text { Females }\end{array}$ & .11 & .05 & .05 & .10 \\
\hline Home drinkers $(n=44)$ & .15 & .05 & .03 & .09 \\
\hline Public drinkers $(n=181)$ & -.03 & -.03 & -.12 & -.08 \\
\hline \multicolumn{5}{|l|}{ Both settings $(n=51)$} \\
\hline at home & -.19 & .08 & -.16 & -.13 \\
\hline in PDP & $-.32^{\text {*4 }}$ & $-.15 t$ & $-.36^{\text {*45 }}$ & $-.39 * *$ \\
\hline
\end{tabular}

\section{Differences on Social Integration between the Drinking Categories}

Contingency tables with chi-square statistics were used to examine the differences in indicators of social integration between the three drinking categories (Table 7.4). The results varied by gender. Male 'home drinkers' had less often a best friend, acquired fewer friends over time, had fewer experiences with intimate relationships, reported more often feelings of loneliness and spent less time with friends than the males in the other two categories. Notice that the most significant differences were apparent between the 'home drinkers' and the 'drinkers in both settings.' Although the trends for girls were comparable to the findings for boys, only for two variables (i.e., 'discussing personal issues' and 'hours with friends') were small differences ( $p$ $<.10)$ found between the three categories. 
Table 7.4. Indicators of Social Integration and Drinking Categories by Gender.

\begin{tabular}{lcccc}
\hline & $\begin{array}{c}\text { Home } \\
\text { drinkers }\end{array}$ & $\begin{array}{c}\text { Public } \\
\text { drinkers }\end{array}$ & $\begin{array}{c}\text { Both } \\
\text { setrings }\end{array}$ & $\begin{array}{c}\text { Sign. } \\
\text { chi-square }\end{array}$ \\
\hline Males & 78.9 & 86.5 & 97.1 & $p<.001$ \\
Best friends & 62.0 & 62.4 & 73.5 & $p<.10$ \\
No. friends & 47.9 & 48.9 & 66.7 & $p<.01$ \\
Never had a partner & 61.4 & 60.4 & 67.4 & ns \\
Discussing pers. issues & 77.5 & 72.7 & 85.8 & $p<.01$ \\
No feelings of loneliness & 52.1 & 70.9 & 74.3 & $p<.01$ \\
Hours with friends (s 8) & & & & \\
Females & 82.2 & 90.7 & 90.4 & $\mathrm{~ns}$ \\
Best friends & 63.6 & 61.2 & 73.1 & $\mathrm{~ns}$ \\
No. friends & 66.4 & 76.8 & 67.3 & $\mathrm{~ns}$ \\
Never had a partner & 80.0 & 84.2 & 94.2 & $p<.10$ \\
Discussing pers. issues & 73.3 & 80.3 & 88.5 & $\mathrm{~ns}$ \\
No feelings of loneliness & 48.9 & 56.3 & 69.2 & $p<.10$ \\
Hours with friends (s 8) & 4 & & & \\
\hline
\end{tabular}

\section{DISCUSSION}

The current study shows that (a) within the category of adolescent male 'home drinkers', consumption levels are related to poor psychological well-being and (b) male "home drinkers" are less socially integrated in a peer network. The characteristics of this category as well as the processes behind the differences remain, however, unclear. Are some youngsters frustrated in their attempts to belong to a social group and try to forget their sorrows at home, or are those who drink at home more likely to be deviant and therefore less attractive to peers? Alternatively, are their drinking habits shaped by parental alcohol misuse which provided an example of drinking to cope? The explanations we offer in this discussion section for our findings should be seen in the light of the explorative character of this study. The cross-sectional design, the limited data on underlying mechanisms, and the small numbers in some drinking categories leave no room for definitive conclusions.

In this study, drinking in public drinking places appears not to be related to stress and low self-esteem. Among those who drink only in public drinking places, or drink both at home and in public drinking places, heavy drinking cannot be interpreted in terms of the reduction of negative feelings and emotions. This seems to contradict studies that report that higher levels of alcohol consumption are related to distress and low self-esteem (e.g., Dolcini \& Adler, 1994; King et al., 1992; Wills, 1994). However, cultural differences in drinking patterns might account for the variation in findings between our Dutch study and North-American studies. For example, the legal age to purchase alcohol is higher in the United States, which is 
likely to result in differences in drinking situations and drinking patterns (for research on 'binge drinking', see Schulenberg et al., 1996). In addition, the relationships between distress and alcohol vary by age and educational level. For instance, O'Callaghan and Callan (1992) explored the relationships between alcohol use, drinking context and reasons for drinking in a sample of 122 Australian college students (18-20 years old). In their study, the most common drinking location was the private home where students drank mostly wine together with some friends. Due to the differences in samples in the O'Callaghan and Callan study and our study (college students versus high school students), the reasons young people have for their drinking and the importance of specific contexts of drinking variate. So the findings are difficult to compare. Both studies, however, show that it is relevant to trace categories of youngsters for whom drinking does not necessarily hold a 'normative' peer activity, but is associated with stress and low self-esteem.

Those who drank exclusively at home were less integrated in peer networks, reported less often involvement in a steady relationship and had more feelings of loneliness compared to those who (also) drank in public drinking places. These effects, which were found only for males, might imply that drinking with peers is not only perceived to be related to enhanced sociability (Brown, 1991), but does indeed have some social benefits for teens" functioning in peer networks. Windle and Windle (1996, p. 551) stated that "... a supportive social environment may protect, or buffer, adolescents from the adverse impact of stressful events..." Since the findings in the current study clearly showed that there are no differences between the drinking categories in absolute levels of stress and self-worth, Windle and Windle's expectation might explain the non-significant relationships between drinking and well-being for drinking in outdoor settings. In other words, although the three drinking categories did not differ in stress levels, it is possible that a good peer network prevents individuals from drinking to cope with stress and low selfworth.

In contrast to males, no negative effects of drinking were apparent for females drinking exclusively at home. ${ }^{4}$ One of the explanations is that young women seek other ways for handling negative emotions. In a review of studies on psychotropic drugs, Cooperstock and Parnell (1982) concluded that women use tranquillizers more often than men. Additionally, Goddard (1990) stressed that girls smoke more frequently than boys in middle adolescence. Thus, perhaps, among female adolescents part of the solution to cope with negative feelings is found in tranquillizers, sleeping pills and cigarettes. A statistical explanation is that the absolute levels of alcohol use were rather low among females who drank at home. This low variation in consumption levels might be responsible for the nonsignificant findings.

One of the most important outcomes from the current study is that examination of the relationships between alcohol and well-being should include (a) the context of use and (b) selection effects. It shows that analyses for the total 
sample, even when the analyses were conducted by setring, do not provide much insight. Only when separate categories of drinkers were distinguished, were significant relationships between level of alcohol consumption and psychological well-being found in one particular category (males who drink only at home). Mann et al. (1987) underscored the importance of distinguishing categories of high-risk adolescents. The authors stated that high and low risk categories (defined on the basis of parental alcoholism and personality characteristics) might not only differ in mean scores on particular variables. Different factors and mechanisms might be involved, resulting in different pathways leading to drinking in separate populations (Mann et al., 1987, p. 416). Better understanding of the psychological processes in relevant categories enhances the chances of construction of effective programs to prevent adolescent alcohol misuse. Other reseachers differentiated high and low risk groups by indicators of deviance and school performance (see Eggert \& Herting, 1993). They found strong variations in drug involvement comparing dropouts and "typical" youth. In the present study, we aimed to make a first step in identifying risk-prone adolescents by looking at the purposes of drinking in different contexts.

It would seem to be worth while to examine the role of coping responses (e.g., Endler \& Parker, 1994; Folkman \& Lazarus, 1988) to gain insight into the mechanisms behind the relationships between drinking, stress and self-esteem for the home drinkers. For instance, emotion-focused coping (i.e. blaming oneself, worrying, inappropriate regulation of emotions) towards stressful events such as inadequate social skills or problems with peers, might not only lead to social dysfunctioning (Windle \& Windle, 1996; Endler \& Parker, 1994) but also to alcohol and drug use (Cooper, Russell \& George, 1988). On the other hand, those with a problem-solving coping style might try to deal with problems more effectively by entering peer-associated and age-normative situations, such as pubs, discos and youth centers. We intend to examine the relations between social support, coping styles, psychological well-being and the context of alcohol consumption in the near future.

Most studies on parental misuse and alcoholism and youngsters' drinking have shown an enhancing effect of parental (i.e. father) misuse on the drinking habits of their off-spring (e.g., Pulkinen \& Pitkanen, 1994). It is likely that children learn about the reasons and norms associated with drinking by observing parental habits. Information about appropriate and inappropriate reasons and settings for drinking (Wilks \& Callan, 1987) induces for a large portion of young people relatively normal drinking behavior. Nonetheless, it is possible that especially parents who drink to alleviate feelings of stress and low self-worth are setting a bad example to their children. Research is therefore warranted to examine how parental drinking and reasons for drinking are related to drinking contexts of both parents and their off-spring. Since we do not have data on drinking motives, coping styles, personality traits or parental alcoholism, it is not possible to trace the different pathways leading to certain drinking styles. 
The present study is limited in some respects. First, its cross-sectional nature limits the understanding of the dynamic processes in the turmoil of the adolescent years. We cannot be conclusive on the position of male "home drinkers'. For some of them, drinking at home may be a temporary phenomenon - they might become socially well-adjusted people a few months or a few years later. Nonetheless, for others it may signify the starting point of a problematic drinking career. It would be necessary to follow a group of male late adolescents for some years to look into these processes in more detail. Second, the low prevalence of adolescents drinking exclusively at home results in rather small numbers of subjects in this category. In future research, it is recommended that the focus should be on larger samples of adolescents. Third, although it is assumed that public drinking places and parental homes are important drinking situations for youngsters, other drinking settings, such as friends' homes, on the street or at work are potentially relevant in studying the effect of context. Fourth, to be more precise about which aspects of youngsters' social life are varying for 'home drinkers' and other categories, scales assessing sociability, quality of social relationships, social anxiety and loneliness should be included in a questionnaire. It would be fruitful to carry out qualitative interviews with subjects in these three categories focusing on parental behaviors, stressful events, coping mechanisms and social functioning. A second step might be the development of a quantitative prospective study following late adolescents into young adulthood.

\section{NOTES}

${ }^{1}$ Based or:

Engels, R.C.M.E., Knibbe, R.A., \& Drop, M.J. (1998). Why do late adolescents drink at home? A study on psychological well-being, social integration and drinking context. Accepted for publication in Addiction Research.

An earlier version of this paper is presented at the 24th Annual Alcohol Epidemiology Symposium of the Kettil Bruun Society for Social and Epidemiological Research on Alcohol, Florence, Italy, 1998.

${ }^{2}$ There were only a few persons who drank exclusively at home. Therefore, the three drinking categories were constructed as follows: those who drank at home and consumed only a few glasses per week (i.e., boys $\leq 5$ glasses; girls $\leq 3$ glasses) outside the parental home were defined as 'home drinkers', those who drank only outside the parental home were defined as 'public drinkers' and those who drank at home and more than a few glasses outside home were defined as 'drinkers in both settings'. Notice that the classificarion is exclusive; an individual can only be allocated to one category.

${ }^{3}$ It is important to mention that it was not expected that, in general, young people who drink at home experience more stress and feelings of low self-esteem than the other categories - we hypothesized their drinking levels to be correlated with levels of psychological well-being. 
Oneway-analyses demonstrated that there were no significant differences of stress and selfesteem between the three drinking categories.

4 Unexpectedly, within the category of young women who drank at home and in public drinking places, levels of consumption were positively related to well-being. We do not have explicit explanations for these findings. One reason could be that girls who are raised to be free, independent and emancipated young women are more likely to function fairly well in social groups and are more used to drinking alcoholic beverages. For those girls, drinking might not be seen as a way to deal with negative feelings but as an expression of independence and well-being. 


\section{8}

\section{PREDICTABILITY OF SMOKING IN ADOLESCENCE: BETWEEN OPTIMISM AND PESSIMISM ${ }^{1}$}

Many people still take up cigarette smoking in teens in the United States (Johnston, O'Malley \& Bachman, 1996) and in European countries (European Bureau for Action on Smoking Prevention, 1994). Recent findings from household surveys in the UK (Office for National Statistics, 1997) and the US (U.S. Department of Health and Human Services, 1997) even showed a small increase in smoking among youth. In the Netherlands, the prevalence of smoking among adolescents has been stable. About forty percent of the 15 to 19 year-olds smoke on a weekly or daily basis (Dutch Foundation of Smoking and Health, 1996).

Insight into the reasons and backgrounds of people who initiate smoking is a central issue in many empirical studies. In their review of theories on the initiation of substance use, Petraitis, Flay and Miller (1995, pp. 79) stated that 'social scientists have many (if not most) of the pieces but do not know how they all fit together". The pieces of a set expected to explain the process of smoking onset are mainly social-psychological factors, such as smoking-specific cognitions, modelling and tolerance of tobacco use in the social environment, personality characteristics and affective states as well as socio-demographic factors. In the field of health education and public health there is optimism about the evidence available on the set variables predicting smoking onset. Particularly peer influences, smoking attitudes, self-efficacy and self-esteem are factors commonly dealt with in theoretically based prevention programmes for youth (e.g., Glynn, 1989; Rundall \& Bruvold, 1988). In general there has been less attention to the relative efficacy of model variables to predict smoking onset. The current study aims to give a critical view of the identification of the precursors of smoking onset. 
According to the findings of cross-sectional studies, much of the variance in smoking behaviour can indeed be explained (see Pederson \& Lefcoe, 1987). The implicit assumption of this type of research is that factors which are found to be associated with smoking behaviour at a specific time-point also predict the onset of use at a later period of time. However, some finely grained longitudinal analyses concentrating on the transition from non-smoker to smoker yield somewhat conflicting findings. Collins et al. (1987) and Graham, Marks and Hansen (1991) showed that it is difficult to predict the onset of smoking over a period of one or two years from a comparable set of factors used in cross-sectional surveys. If a model explains two-thirds of the variance in smoking but only predicts a few percent of the variance in smoking over an extended period of time, the relevance and practical value of this model is limired (Randall \& Wolff, 1994). Unfortunately, the boundaries of predicting smoking onset are rarely questioned. Nonetheless, it would provide essential information for practice. Smoking prevention programmes concentrate on children before they start to smoke (see Flay, 1985; Oei \& Fae, 1987). It is, therefore, important to know to what extent the factors which the prevention programmes aim to influence are precursors of onset a few years later.

Much of the confusion concerning the value of individual factors and, subsequently, on the predictability of onset is due to differences in research designs and analytic strategies. We used data from a three-wave five-year longitudinal research project of secondary schoolchildren (measurements in 1989, 1991 and 1994) to illustrate how predictability depends on the design and analyses employed.

\section{Cross-sectional versus Longitudinal Studies}

Most studies have used a cross-sectional design to investigate the relations between predictor variables and smoking behaviour. A drawback of cross-sectionall research is that only the correlates of differences in smoking status can be discussed. More definite conclusions can not be drawn about the causal interpretation of an empirical association (Conrad, Flay \& Hill, 1992; Chassin, Presson, Sherman, Corty \& Olshavsky, 1984). It remains unclear to what extent, for example, a change in attitude is accompanied by a change in behaviour rather than preceding it (Sutton, 1992). In fact, no information is gathered on the variables associated with the onset of cigarette use. Thus, cross-sectional studies are helpful in generating hypotheses but do not make firm conclusions about predictors of onset possible.

One of the features of prospective research is that more definite conclusions can be drawn about the causality of an empirical association. A prospective design rules out the possibility that an association between predictor $X$ at time 1 and smoking behavior at time 2 is due to causal effect of time 2 behavior on the time 1 predictor. They do not rule out the possibility that the association is due wholly or partially to some other variable(s). Nevertheless, the development of prospective designs to examine precursors of smoking onset does not necessarily exclude 
difficulties with the interpretation of outcomes. A major limitation of many longitudinal studies concerns the use of cross-sectional analytic strategies; pre-test scores on predictor variables being related to post-test smoking status. Another widely employed method is to include pre-test smoking status as one of the determinants of post-test smoking status. A disadvantage of the latter approach is that only changes in smoking status are predicted. The conclusions from this type of analyses are less specific because different smoking groups are included. The causes of smoking onset might be different from the causes of maintenance or quitting. Some researchers stated, therefore, that the process of transition from one smoking category to another should be predicted (Ary \& Biglan, 1988; Chassin et al., 1984; Leventhal \& Cleary, 1980). To predict onset, one should select the nonsmokers at a given time-point and investigate the factors preceding the change from non-smoking to experimental or regular smoking.

Longitudinal studies on potential determinants of smoking have mostly used a design with a baseline measurement, with a follow-up survey being carried out and a sample of secondary schoolchildren being followed (e.g., Ahlgren et al., 1982; Ary \& Biglan, 1987; Charlton \& Blair, 1989; Chassin et al., 1984; Ennett \& Bauman, 1994; Goddard, 1990; Graham et al., 1991; McNeill et al., 1988; Morgan \& Grube, 1991; Urberg, Cheng \& Shyu, 1991; Warheit et al., 1995). Some of these researchers carried out analyses in which the effects of factors measured at time one on smoking at time two were examined for the total sample. Earlier smoking status accounted for most of the variance in changes in smoking status at a later wave (see Collins et al., 1987; Graham et al., 1991; Urberg et al., 1991). This effect was strongest in the case that the time-lag between the waves did not carry over one or two years (see Pederson $8 x$ Lefcoe, 1987, for a study over 5 and 8 years). The effects of other variables, after earlier tobacco use entered the equation, were modest. In most cases, the explained variance by predictor variables did not exceed ten percent.

Studies concentrating on the transition from non-smoker to smoker, however, did not offer a completely different picture. Although some factors are consistently found to be predictive of onset, such as environmental factors, smoking-related cognitions and socio-demographics (see the review of longitudinal studies by Conrad et al., 1992), the absolute effects were rather small. Furthermore, one should bear in mind that most of these studies followed youngsters for only a short period of time. The limited time-span of the majority of prospective research (mostly a follow-up after one year) and the low predictive value of the models tested in these studies leads to serious doubts about what we know about the factors leading to smoking onset.

\section{The Present Study}

The central theme in this study is the predictability of adolescent smoking behaviour. We evaluated the ability of social-psychological and socio-demographic 
factors to predict smoking by a comparison of cross-sectional and longitudinal findings. In the cross-sectional analyses, we examined to what extent variables were multivariately associated with smoking behaviour. With respect to the prospective analyses, two different strategies were carried out. First, one analysis was conducted in which changes in smoking status were predicted by factors (including smoking behaviour) measured at a previous wave. Within a second analysis, the transition from non-smoker to current smoker was examined. In order to determine whether a particular variable was related to smoking onset, the analysis was restricted to the selection of non-smokers at a preceding wave.

A set of variables was employed which consisted of smoking-related cognitions, such as attitudes, self-efficacy and future intentions, social influences, such as smoking by the parents, a best friend and the peer group, and sociodemographical factors, sucth as gender, family structure and educational level. Although the primary goal of this study relates to the predictability of smoking, attention was paid to differences in strength of particular variables in the analyses. For instance, it is possible that some factors were associated with smoking in the cross-sectional analyses but were not predictors of smoking onset (or vice versa). Nevertheless, for reasons of clarity, we discuss only the most remarkable differences in findings. In addition, we did not test explicit hierarchical models with specified interrelations between variables by two reasons. ${ }^{2}$ There is no consensus in the literature about which model (within the set of variables used here) should be applied (see Petraitis et al., 1995). Thus it is rather arbitrary to choose between one or the other. In this study, therefore, a simple model was tested without distinction of proximal and distal variables. Notice that our main aim is to look for predictability -- we assume that, in terms of explained variance, variations in theoretical models will not lead to completely different outcomes. Furthermore, attention is focused upon differences in predictor variables which "explain" why some youngsters start to smoke (and which can be implemented in intervention programs) and predictor variables for which the link with smoking is more difficult to explain (e.g., gender, education).

Prevalence figures clearly show that only a small proportion of children in the 10 to 14 age category use cigarettes (Dutch Foundation of Smoking and Health, 1996). The increase in prevalence of current smoking is highest in the 15 to 19 age category. To gain insight into the precursors of onset, one should focus on the period in which most youngsters start to smoke. Therefore, the longitudinal part of this study dealt with smoking onset measured at the third wave in which subjects were at the ages of 17 or 18 . The predictor variables were measured at the first wave (mean age 12.4) and the second wave (mean age 14.4) five and three years, respectively before the third wave took place. ${ }^{3}$ 


\section{METHOD}

\section{Procedure, Attrition Analyses And Sample Characteristics}

In 1989 (T1), this study started with a sample of students from first and second grade of secondary education in the Netherlands. A selection of 36 schools differentiated by educational level ${ }^{4}$ and region participated in the study. At the first measurement, the questionnaires were administered in the classrooms in the presence of a teacher and the questionnaires were returned in a sealed envelope. No explicit refusals were recorded; non-response was exclusively due to absence (e.g., sickness) at the day of assessment. 1,454 adolescents participated in the first wave. In the autumn of 1991 (T2) and 1994 (T3), forms were sent to subjects' homes. Subjects who did not respond to mailed reminders were approached by phone to complete an interview. The response rates were $82 \%$ in both follow-up surveys. A total of 1,063 adolescents $(73 \%)$ were enrolled in all three waves.

A logistic regression analysis was conducted to compare participating subjects and the drop-outs with data of the first measurement as predictors of drop-out. Dropouts $(n=391)$ appeared to be older, less educated, attended a church less often and had a father of Dutch origin less often compared to remainers $(n=1,063)$. Small differences $(p<.10)$ were found for sex and intensity of cigarette smoking. Dropouts were more likely to be male and reported a higher number of cigarettes smoked than remainers. No differences $(p>15)$ were found for frequency of drinking and smoking, or intensity of drinking.

The sample participating at all three waves consisted of 520 girls (49\%) and 543 boys $(51 \%)$. At the first wave, the mean age was $12.4(S D=.7)$. In 1989 , onethird of the students were enrolled in vocational school, and $64 \%$ in high school. Most youngsters ( $85 \%$ ) lived with two parents (not including stepparents). Ninetythree percent had a father or mother of Dutch origin.

\section{Measures}

The dependent variable smoking behaviour was included in all three waves. The predictor variables $(n=19)$ were measured identically at each wave with a few exceptions. At the third wave, the indicators of level of education, employment and pocket money were adjusted to the rising age of the sample. Furthermore, no questions were asked about exposure to prevention programmes at school or in the media at the third wave. For waves one and two, an identical (in number of variables and in operationalizations) set of predictor variables was used. Those subjects with at least one missing value on the variables were omitted from the analysis.

Subjects were asked to report their life-time smoking with the question: 'Have you ever smoked a cigarette, even a few puffs?' This item was used to indicate 
whether they had experimented with cigarettes ( 1 yes, 2 no). Responses on the item smoking in the last four weeks were used as an indicator of the frequency of use with the options: not in the last four weeks, less than weekly, at least weekly and daily. This item was transformed into (0) non-smoking and (1) current smoking (i.e., smoking in the last four weeks).

A subset of five questions, taken from a 24-item attitude scale of De Vries et al. (1986), measured different kinds of aspects associated with smoking. An example of a question was: 'If I smoke (or would smoke) ... I (wrould) strongly dislike the taste (1), or (would) strongly like the taste (6)'. A similar kind of format was used for: passive smoking by others, alleviation of boredom, shortness of breath, and a smart thing to do. Beliefs were measured on a 4-point scale ( 3 items) and 6-point scale ( 2 items). Cronbach's alphas were .62 (T'1), .69 (T'2) and .70 (T'3).

Three items were abstracted from a 9-item self-efficacy scale (De Vries, Dijkstra \& Kuhlman, 1988) and measured the confidence subjects had in the ability to become (or stay) non-smokers and their confidence to refuse a cigarette when one was offered. An example of a question was: "To become (or to stay) a non-smoker is ... very difficult for me (1), or very easy for me (7). Cronbach's alphas were .64 (T1) and .83 (T2). This concept was measured with two items at wave three $(r=.75)$.

A one item indicator of adolescents' future intentions to smoke was used. For the question 'Do you intend to start smoking (or to maintain smoking)?', response categories ranged from (1) absolutely not to (7) absolutely.

For the father, mother, eldest brother, eldest sister and the best same-sex friend, subjects were asked if they considered them to be smokers. Scores were dichotomized to (1) no/do not know and (2) yes. Peer group smoking was measured by the proportion of smokers in the peer group on a 5-point scale which ranged from no one/do not know (1) to all members smoke (5).

Questions on gender, level of education, living arrangement, having a job, church attendance, pocket money and origin were included. Two indicators of awareness of smoking prevention were employed. First, subjects were asked if they had followed any health education on smoking during primary school years. The response categories were (1) never/do not know, (2) once and (3) more than once. In addition, they were asked about whether they noticed activities in the media targeting on smoking among children. This item had response categories (1) never/do not know, (2) once and (3) often.

\section{Data Analyses}

Multivariate logistic regression analyses were conducted for the cross-sectional and longitudinal data. ${ }^{5}$ 'The dependent variable was smoking in all analyses with $0=$ 'non-current smoking" and $1=$ "current smoking'. Variables were included in analyses by a forward selection procedure and the likelihood ratio criterion was used to determine which variables should be added to the model. A probability of 
.05 was used for a variable to enter the equation and a probability of . 10 to remove a variable from the equation. The $R_{L}^{2}$, which is an estimate of the efficacy predicted by the model variables (see DeMaris, 1995), was indicative of the explained variance. The models reported in the tables provides estimates of the effects while controlling for the other variables in the model.

The relations between predictors and smoking on a cross-sectional level were examined for the three waves. ${ }^{6}$ The longitudinal effects of predictors were investigated in two ways. First, the effects of the predictor variables measured at the first wave on smoking at the third wave were examined for the total sample. The smoking status at the first wave was considered as a predictor variable. Another strategy was employed wherein the non-current smokers at the first wave were selected, and the effects on smoking onset at the third wave were investigated. Experimental smoking at wave one was included as a predictor variable. These two types of analyses were also performed for the predictors measured at wave two.

Although it is conceivable that interaction effects between variables were apparent, we only examined the main effects. Moreover, no assumptions were made about possible hierarchical models in the set of predictor variables. It is beyond the scope of this paper to address these issues.

\section{RESULTS}

\section{Changes in Smoking Bebaviour across the Waves}

This prospective cohort which follows young people from their first year in secondary education (12-13-years) until their late teenage years (17-18-years) give a clear picture of the build up of smoking prevalence with age. The sexes did not differ significantly in current smoking at the first wave (11\% for boys and $12 \%$ for girls). However, two years later, more girls $(23 \%)$ than boys $(18 \%)$ were current smolkers $\left(\chi^{2}(1063)=5.13, p<.05\right)$. A sharp increase in smoking prevalence is seen at the third wave for both sexes. More than one third ( $40 \%$ of the boys and $43 \%$ of the girls) indicated to smoke.

\section{Cross-sectional Associations between Predictor Variables and Smoking}

Table 8.1 depicts the odds ratios, $95 \%$ confidence intervals, regression coefficients and estimates of the explained variance of the logistic regression analyses. T'he efficacy of the model variables to explain current smoking was relatively high at each time point. Fifty percent of the variance in smoking in the first wave could be explained by the model variables at the first wave. These estimates were even higher at the following waves: $64 \%$ at the second wave and $71 \%$ at the third. wave.

There were substantial similarities in the three cross-sectional analyses. Scores on attitudes and self-efficacy were most strongly related to current smoking. ${ }^{7}$ Those 
with positive attitudes on smoking and lower self-confidence in resisting external and internal pressures to smoke were more likely to smoke. Smoking by their best same-sex friend and the proportion of smokers in the peer group were also positively associated with current smoking at each time point. Smoking by family members was not related to smoking at $\mathrm{T} 1$ but smoking by the father or mother was associated with smoking at T2 and T'3. Some socio-demographic variables were only weakly related to smoking. Following higher education ( $\left.\mathrm{T}^{\prime} 1\right)$ and being male (T3) were associated with non-smoking.

Table 8.1. Multivariate Analyses of Predictors on Smoking Status: Cross-sectional Analyses

\begin{tabular}{|c|c|c|c|c|}
\hline & Odds ratio & $\begin{array}{l}\text { Regression } \\
\text { coefficient }\end{array}$ & $\begin{array}{c}95 \% \text { Confidence } \\
\text { interval }\end{array}$ & $\begin{array}{c}\text { Explained } \\
\text { variance }\end{array}$ \\
\hline \multicolumn{5}{|l|}{$1989(n=943)$} \\
\hline Attitude $(5-24)$ & 1.39 & $.33^{2 x+25}$ & $1.27-1.52$ & $\mathrm{R}_{\mathrm{L}}^{2}=.50$ \\
\hline Self-efficacy $(3-21)$ & .84 & 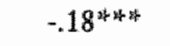 & $78-.90$ & \\
\hline Best friend smokes $(n / y)$ & 3.11 & 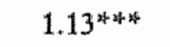 & $1.69-5.72$ & \\
\hline Peer group smoking (1-5) & 1.48 & $.39^{\sin x^{2}}$ & $1.14-1.91$ & \\
\hline \multicolumn{5}{|l|}{ Education } \\
\hline high vs low & 3.72 & $1.31^{\text {sh }}$ & $1.38-10.04$ & \\
\hline high vs average & 4.24 & $1.45^{*}$ & $1.57-11.50$ & \\
\hline \multicolumn{5}{|l|}{$1991(n=900)$} \\
\hline Self-efficacy & .81 & $-.21^{4 \text { 차 } 3 \text { 极 }}$ & $.76-.87$ & $\mathrm{R}_{\mathrm{L}}^{2}=.64$ \\
\hline Best friend smokes & 5.43 & $1.69^{4+3}$ & $2.98-9.87$ & \\
\hline Attitude & 1.41 & 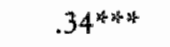 & $1.28-1.56$ & \\
\hline Peer group smoking & 2.05 & $.72^{* * 4}$ & $1.56-2.71$ & \\
\hline Father smokes $(n / y)$ & 1.84 & $.61^{*}$ & $1.03-3.27$ & \\
\hline Brother smokes $(\mathrm{n} / \mathrm{y})$ & 2.18 & $.78^{*}$ & $1.02-4.66$ & \\
\hline \multicolumn{5}{|l|}{$1994(n=850)$} \\
\hline Self-efficacy (2-14) & .60 & $-.51^{+44+4}$ & .54 .67 & $\mathrm{R}_{\mathbb{L}}^{2}=.71$ \\
\hline Atritude & 1.39 & $.33^{2544}$ & $1.26-1.53$ & \\
\hline Peer group smoking & 1.55 & $.44^{4+4}$ & $1.15-2.08$ & \\
\hline Intention to smoke $(1-5)$ & 1.96 & $.67^{2+3+4 x}$ & $1.41-2.72$ & \\
\hline Best friend smokes & 2.30 & $.83^{*+4}$ & $1.69-4.24$ & \\
\hline Mother smokes $(n / y)$ & 2.02 & $.71^{*}$ & $1.12-3.65$ & \\
\hline Sex (male/temale) & 1.84 & $.61^{3 *}$ & $1.03-3.27$ & \\
\hline
\end{tabular}

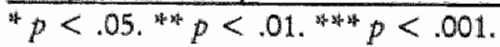

\section{Longitudinal Predictors of Changes in Smoking}

Over a period of five years, the model variables predicted $11 \%$ of the variance in smoking (see Table 8.2). Furthermore, the T2-predictor variables predicted $30 \%$ of the variance in smoking status over a period of three years. Earlier smoking did not affect the smoking status over a five year period according to this multivariate 
analysis. However, over a period of three years, earlier smoking predicted changes in smoking: those who smoked at wave two were almost three times more likely to smoke at the third wave. Differences in attitudes, future intentions and self-efficacy (only for T2-data) were related to current smoking at T3. Modelling effects were apparent for the reference persons mother, sister (T1-data) and best friend (T1-data). Those with a smoking mother, a smoking sister and a smoking best same-sex friend were more likely to smoke themselves. Having a job at T2 was related to current smoking.

Table 8.2. Multivariate Analyses on Variables Related to Current Smoking at the Third Wave: Longitudinal Analyses for the Total Sample

\begin{tabular}{|c|c|c|c|c|}
\hline & OR & $\begin{array}{l}\text { Regression } \\
\text { coefficient }\end{array}$ & $95 \% \mathrm{CI}$ & $\begin{array}{c}\text { Explained } \\
\text { variance }\end{array}$ \\
\hline \multicolumn{5}{|l|}{$1989 \cdot 1994$} \\
\hline Atritude $(5-24)$ & 1.15 & $.14^{\text {thtsor }}$ & $1.10-1.21$ & $\mathbb{R}_{L}^{2}=11$ \\
\hline Intention to smoke (1-7) & 1.26 & $.23^{* 2+4+4}$ & $1.13-1.41$ & \\
\hline Mother smokes $(\mathrm{n} / \mathrm{y})$ & 1.67 & 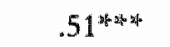 & $1.26-2.22$ & \\
\hline Best friend smokes $(n / y)$ & 1.59 & $.47^{*}$ & $1.06-2.39$ & \\
\hline Sister smokes $(\mathrm{n} / \mathrm{y})$ & 1.85 & $.62^{*}$ & $1.05-3.26$ & \\
\hline \multicolumn{5}{|l|}{ 1991-1994 } \\
\hline Self-efficacy (3-21) & .84 & $-17^{4+3}$ & $.79-.90$ & $\mathrm{R}_{\mathrm{L}}^{2}=.30$ \\
\hline Attitude & 1.14 & 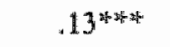 & $1.08-1.20$ & \\
\hline Smoking T2 $(\mathrm{n} / \mathrm{y})$ & 2.90 & $1.06^{5+24245}$ & $1.61-5.23$ & \\
\hline Mother smokes & 1.65 & 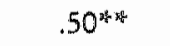 & $1.17-2.32$ & \\
\hline Intention to smoke $(1-7)$ & 1.23 & $.21^{34}$ & $1.07-1.42$ & \\
\hline $\operatorname{Job}(\mathrm{n} / \mathrm{y})$ & 1.51 & $.41^{\text {th }}$ & $1.07-2.13$ & \\
\hline
\end{tabular}

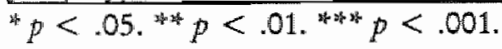

\section{Longitudinal Predictors of Smoking Onset}

Table 8.3 depicts the findings of two logistic regression analyses in which the effects of the predictor variables (T1 and T2) on smoking (T3) were investigated for the selection of non-smokers. Respectively eight percent and fourteen percent of the variance in smoking onset at the third wave could be predicted by six factors. Those who experimented with smoking (i.e., tried smoking but did not smoke on a regular basis at $\mathrm{T} 1$ or $\mathrm{T} 2$ ) were more likely to take up smoking later on. Cognitions such as future intentions, attitude and self-efficacy (only for T2-data) affected smoking onset. In addition, smoking by the mother and sister (T1-data), being female (T1data) and having a job (T2-data) were of predictive value on onset.

It should be mentioned that some of the most robust correlates of cigarette use, namely smoking by a best friend or the proportion of smoking peer group members, did not reach significance in the models predicting smoking onset. The 
effects of smoking family members were small but consistent in cross-sectional as well as longitudinal analyses.

Table 8.3. Multivariate Analyses on Variables Related to Initiation of Smoking at the Third Wave: Longitudinal Analyses for the Selection of Non-smokers

\begin{tabular}{|c|c|c|c|c|}
\hline & $\overline{\mathrm{OR}}$ & $\begin{array}{l}\text { Regression } \\
\text { coefficient }\end{array}$ & $95 \% \mathrm{CI}$ & $\begin{array}{c}\text { Explained } \\
\text { variance }\end{array}$ \\
\hline \multicolumn{5}{|l|}{$1989 \cdot 1994$} \\
\hline Tried smoking $(n / y)$ & 2.04 & $.711^{20 * 4}$ & $1.45-2.86$ & $R_{L}^{2}=.08$ \\
\hline Attitude (5-24) & 1.10 & $.09^{\text {25 } 54+4}$ & $1.03-1.16$ & \\
\hline Mother smokes $(\mathrm{n} / \mathrm{y})$ & 1.59 & 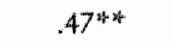 & $1.18-2.15$ & \\
\hline Interition to smoke (1-7) & 1.18 & $17^{* \text { * }}$ & $1.04-1.34$ & \\
\hline Sex (male/fernale) & 1.36 & $.31^{*}$ & $1.01-1.85$ & \\
\hline Sister smokes $(\mathrm{n} / \mathrm{y})$ & 1.88 & $63^{4}$ & $1.00-3.52$ & \\
\hline \multicolumn{5}{|l|}{$1991-1994$} \\
\hline Self-efficacy $(3-21)$ & .84 & $-17^{2}$ & $.78-91$ & $\mathrm{R}_{\mathrm{L}}^{2}=.14$ \\
\hline Attitude & 1.08 & $.08+2+4$ & $1.02-1.15$ & \\
\hline Mother smokes & 1.64 & $.50^{\text {at* }}$ & $1.14-2.37$ & \\
\hline Intention to smoke & 1.26 & $.23^{\text {sit }}$ & $1.08-1.47$ & \\
\hline $\operatorname{Job}(n / y)$ & 1.47 & $.39^{* 2}$ & $1.02-2.13$ & \\
\hline Tried smoking $(\mathrm{n} / \mathrm{y})$ & 1.53 & $.43^{*}$ & $1.02-2.29$ & \\
\hline
\end{tabular}

\section{DISCUSSION}

The present study took a closer look at the efficacy of a set of variables to predict smoking behaviour by using cross-sectional and longitudinal data of a study among secondary schoolchildren in the Netherlands. In general, the predictability of smoking strongly depends on the design and analytic strategy employed. According to the findings of the cross-sectional analyses, one could be optimistic about the explained variance of the set of predictor variables. The explained variance ranged from $50 \%$ in 1989 to $71 \%$ in 1994 . However, the longitudinal analyses displayed a different picture. Prospective analyses for the total sample in which earlier smoking was incorporated as a predictor variable explained $11 \%$ of the variance in smoking over five years and $30 \%$ in three years. However, these analyses predicted changes in smoking instead of onset. The most precise analyses in which the onset of smoking was predicted showed that $8 \%$ of the new smokers could be predicted by the model variables. Over a period of three years, this percentage improved to fourteen. The longitudinal findings with respect to the prediction of smoking (onset) did not differ much from other research which used a 'similar' set of variables (see Collins et al., 1987; Graham et al, 1991; Pederson and Lefcoe, 1987). In conclusion, our results showed that cross-sectional analyses highly overestimate the value of predictor 
variables and that the most informative prospective analysis shows that only a few percent in smoking onset can be predicted. Before we take a rather pessimistic view on the efficacy of variables to predict the onset of smoking, the question should be asked what kind of effect sizes we could expected. Initiation of smoking takes place in turmoil of adolescence which is characterized by many personal and social changes. Using a prospective design with relatively long periods between the waves (two and three years), it might be difficult to capture, and understand, the dymamics. in which uptake of risky behaviors takes place. In this respect, an effect size of $14 \%$ might not considered to be that low. According to Rosenthal (1990) and Cohen (1977), even very small effect sizes can have theoretical and practical utility. Thus, although the effect sizes imply that much remains unknown about why youngsters start smoking, they still offer some tools for prevention efforts.

Smoking-related cognitions, such as attitude, self-efficacy and future intentions were consistently included in the regression equations. Those with positive attitudes toward smoking and with high intentions to smoke were more likely to initiate smoking over three and five years. Self-efficacy was not included in the multivariate analyses predicting smoking over a period of five years, but was a predictor of onset over three years. Although the absolute effects of these factors were small, they seem to be the relatively strongest predictors of smoking onset (in our set of variables). An practical advantage is that these variables are open for change - interventions can be developed in order to change cognitions. Nonetheless, the prominent differences in effects between cross-sectional and longitudinal findings point to some alternative explanations. The high correlation between cognitions and smoking measured at a given time point might reflect the effects of behavior on attitudes and vice versa. If the possible effects of earlier experiences with smoking are minimised, as we did in the longitudinal analyses for the sample of non-smokers, the effects of attitudes on behaviour are less convincing. Another interpretation deals with the duration of the attitude effects. It is possible that teenagers alter their attitudes and start to smoke in a shorter period of time than expected. So these two events might be so close together that longitudinal studies with more than a few months between the waves can not capture this process (Goddard, 1990; Sutton, 1992).

Cross-sectional analyses showed that smoking by peers is strongly related to the smoking status of the respondent. However, peer influences were in most cases not significantly related to changes in smoking behaviour or to smoking onset. "This discrepancy is reported in other studies (Bauman \& Ennett, 1996; Kandel, 1978). Selective association, distortions in the perception of peer smoking and instability of friendships are proposed as explanations for this phenomenon. Some studies have shown that adolescents seek contacts with peers who have similar opinions and behaviours. Longitudinal analyses in which these "birds of a feather" effects were controlled for demonstrated only small effects of peer influences (Engels, Knibbe, Drop \& De Haan, 1997; Bauman \& Ennett, 1996). Furthermore, smoking teens 
tend to overestimate the prevalence of smoking in their inner circle of friends, peers, or class members (Sherman et al., 1983). This false consensus effect might partly account for the strong similarities of smoking behaviour within friendships. Finally, friendships change rapidly in adolescence. It might not be surprising that the impact of friends assessed at a specific moment of time does not carry over a period of a few years. In this respect, the findings for parental smoking are worthwhile. The problems with causality are not prominent - it is not that likely that children strongly affect parental smoking or that selection effects occur. Thus, the similarities of smoking in parent-child-relationship deserves (renewed) attention in theoretical models as well in prevention efforts.

Studies with a design of three or more waves can provide insight into the relationship between the time interval between measurements and predictability of smoking onset. From our findings, one might conclude that the predictive value of factors did not sustain over time. Research which has provided information concerning temporal contiguity has also described that the predictability of smoking declines with time (Collins et al., 1987; Guy, Smith \& Bentler, 1994; Pederson \& Lefcoe, 1987; Stein, Newcomb \& Bentler, 1996) while others have not found any support for this assumption (Semmer et al., 1987).

We did not tested an explicit hierarchical model with specified intercorrelations between variables because there is no consensus in the literature about which model (within the set of variables used here) should be applied (see Petraitis et al., 1995). However, the choice for forward regression analyses has its pittfals. For instance if a particular variable correlates with another variable which has explanatory power, it may be excluded from the model although it correlates with smoking on the univariate level.

The estimates of the explained variance in the cross-sectional analyses were dissimilar for the three waves. The correlations of the determinants with smoking became stronger with the years. A possible explanation lies in the design with repeated measurements. Filling out identical items on more than one occasion might affect the inter-relations between items because people remember their earlier answers. The time-lag between waves, however, makes this unlikely. Another explanation refers to the relevance of the topic. Perhaps children aged 12 to 14 do not think seriously about the pros and cons of smoking, or they are not confronted with situations in which they must demonstrate their efficacy to deal with cigarette offers adequately.

One of limitations of the current study is that analyses were only performed for the total sample. Predictor variables might vary in their impact on smoking onset for different categories, such as gender (Flay et al., 1994) ethnic background (Warheit et al., 1993) or educational level (Chassin et al., 1984). We did not perform the analyses for subpopulations. However, if prevention programmes are directed towards certain categories, specific information on the determinants of smoking onset in these categories might be more helpful than some general information 
about the overall population.

Within this field of research, many studies have concentrated on personality characteristics (e.g., sensation seeking, extraversion, neuroticism) and affective states (low self-esteem, depression, anxiety) as precursors of smoking behaviour (see review by Petraitis et al., 1995). These type of variables were not included in the current study. It would be worthwhile for researchers focussing on other predictors of onset to test the research questions of this paper.

\section{Directions for Research and Prevention}

The gap between the outcomes of cross-sectional and longitudinal analyses allows several explanations to be given. First, the processes leading to smoking habits might take place in a very short period of time which can not be identified by longitudinal research with intervals of more than a few months between the waves. For every individual, there are a few critical moments in adolescence in which cigarette use turn up as a significant topic and one must decide whether to try a cigarette or not. Moreover, it is conceivable that people express a negative view on the advantages of smoking for some years but at a particular moment, when they are seriously confronted with the habit, they change their attitudes and intentions. Additionally, many teenagers smoke their first cigarette in the presence of peers, friends or class mates (Friedman, Lichtenstein \& Biglan, 1985). Friendships are, however, in most cases very unstable. In the adolescent years, people have many opportunities to meet peers and establish new friendships (e.g., by entering new classes or developing activities such as sports, pub-going or clubs). One of the drawbacks of most longitudinal research (including our cohort study) is that changes in orientations, activities, social relationships and smoking-specific cognitions in the period between the measurements are not assessed. This points to a need for longitudinal studies which monitor a group of young people for an extended period of time. Particularly the use of quantitative as well as in-depth qualitative data (individual interviews and focus group interviews) should enhance the understanding of the process of smoking onset.

Differences in measurements of predictor variables and dependent variables, sample characteristics, attrition rates, analytic strategies and time-intervals between waves make it barely possible to compare the outcomes of longitudinal studies. As mentioned by Conrad and co-workers (1992, pp. 1722), most studies have not included factor analysis or other assessments of scale properties. The use of predictor variables of unknown validity and reliability leads to serious complications if one wishes to examine the value of variables to predict smoking across studies. Furthermore, presentation of multivariate analyses on different sets of items leaves no room for an accurate comparison across studies. Researchers should be encouraged to present univariate analyses and scale properties.

Much of the work on adolescent smoking is based on the adolescents' 
perceptions of the acts and thoughts of others. Asking persons in the immediate social environment of adolescents themselves about these issues can result in inaccurate conclusions about the value of some predictor variables (see Bauman \& Ennett, 1996). In addition, significant others could provide more information on subjects" functioning in peer groups and in the family and about the specific moments and situations in which influence processes lead to changes in smoking.

Primary prevention is mostly aimed at young people who have not used substances yet. One tries to catch the thief before he or she even thinks about stealing. By offering information to encourage thinking about the pros and cons of smoking, and teaching skills to resist peer influences, youngsters are expected to have sufficient tools to say no to cigarettes. There are a few disadvantages to this approach. Our results show that differences in attitudes, self-efficacy or peer smoking do not account for much of the variance in smoking onset later on. Following this argument, it is not very surprising that the effects of actual intervention and prevention programmes are marginal (see Bruvold, 1993). In addition, according to Fillmore (1996, pp. 11) school-based educational programmes are poor competitors to peer influence in these experimental years of life. This suggests that to improve the effectiveness of prevention one should continue smoking education throughout the primary and secondary school years. Furthermore, much attention is focussed on peers as the most influential party. Our findings show that parents might be mistakenly neglected in most programmes. It would be interesting to explore how parental influences can be taken into account in prevention, and whether acknowledgement of family factors would actually improve the effectiveness of prevention (Klitzner, Gruenewald \& Bamberger, 1990).

In conclusion, this study illustrates that what we think we know about the processes leading to smoking onset strongly depends on the research design and analytic strategies employed. Cross-sectional analyses showed high correlations between explanatory variables and smoking. Nonetheless, the most informative longitudinal analyses in our study indicated that only a few percent of new smokers can be predicted by the model variables. We are aware of the fact that reducing findings to percentages of explained variance is not the cure. However, it does focus attention on the limitations of the present models and research. Eventually, this might lead to alternative ways of thinking about the endpoints, insights into the mechanisms behind smoking, and research methods.

\section{NOTES}

'Based on:

Engels, R.C.M.E., Knibbe, R.A., \& Drop, M.J. (1998). Predictability of smoking in adolescence: Berween optimism and pessimism. Accepted for publication in Addiction.

Engels, R.C.M.E., Knibbe, R.A., \& Drop, M.J. (1997). Wie beginnen er met roken? Een kritische studie naar de woorspelbaarheid wan rookgedrag bij jongeren. In: $D$. Daamen, $A$. Pruym, W. Otten \& R. Meertens (Eds.), Sociale Psychologie en baar toepassingen (pp. 159-171). 


\section{Delft: Eburon.}

${ }^{2}$ A reviewer suggested a hierarchical analytic strategy in the analyses instead of the stepwise method as we used in this paper. In essence, there are two reasons for our approach. First, it should be stressed that the main goal of this paper is the more strategic question how much we know about predictors of smoking. The relative effect of, for instance, attitudes or self-efficacy in a specific analysis is of less importance for us. We also think that, within our data-set, other models would not show really different outcomes in terms of explained variance. Second, it is rather arbitrary how to organize your hierarchical analyses because of the confusion in the literature. Bentler and Speckart (1979) assumed that attitudes and modelling are on the same level and are both proximal predictors of intentions. However, Flay et al. (1994) proposed a model in which it was hypothesized that there are no direct effects of perceived behavior of significant others but that it runs through social norms, attitudes and self-efficacy. In addition, researchers have different views on how to deal with experimental smoking. Most of them, as is mentioned in the introduction, set up an analysis in which previous behavior is entered in the first step. Nonetheless, De Vries et al. (1995) stressed that the effects of habits and addiction run through cognitions, and that there is no direct impact of previous behavior. So they entered previous behavior in the last step after the cognitions and social influences. Another problem is how to deal with socio-demographic variables. In terms of Petraitis et al. (1995) and the Fishbein and Ajzen approach, variables such as sex, age and SES are distal variables. It is postulated that there is no direct path between these kind of variables and substance use, but that their impact runs through other, more proximall factors. In contrast with this approach, along with some sociological traditions, sex and age are entered first and, in a second step, the other predictor variables (see for example Urberg et al., 1991). Because of these wide-ranging and quite different standpoints in the literature, we decided to use a somewhat 'a-theoretical' approach in which no underlying structure and model within the predictor variables was constructed (as also done by Chassin et al., 1991; Charlton \& Blair, 1989 and others)(Note that the above mentioned references are examples of an approach).

${ }^{3}$ A third longitudinal analysis could be performed by examining the effects of predictors measured at T1 on smoking at T2. Since the present study concentrated on the precursors of the uptake of smoking, and most adolescents started to use at the third wave, this analysis was not presented.

* Two main types of secondary education can be distinguished in the Netherlands: vocational schools preparing students for jobs, such as electrician, carpenter or housewife, and high schools for continued and more specialized education at colleges and universities.

${ }^{5}$ In many studies, multiple regression analyses have been used to investigate the effects of predictor variables (Conrad et al., 1992). One might argue whether the frequency of smoking behavior can be considered as an interval scale (see Pederson \& Lefcoe, 1987). In most cases, subjects are positioned on the extremes of the scale: one smokes on a regular basis or one does not smoke. The dichotomous character of the variable led us to decide to test hypotheses by means of logistic regressions.

${ }^{6}$ Since the telephone interview at wave three did not contain all predictor variables, the crosssectional analyses were restricted to those who filled out a questionnaire $(n-958)$. 
Chapter 8

" The fact that that some variables were measured on a dichotomous scale (e.g., parental smoking, sexe) whereas other wariables were assessed on a ordinal scale (e.g*, attitudes, selfefficacy) makes it difficult to compare the odds ratios. 


\section{ANTECEDENTS OF SMOKING CESSATION AMONG ADOLESCENTS: WHO IS MOTIVATED TO CHANGE? ${ }^{1}$}

Traditionally, determinants of smoking onset by young people have been one of the central themes in social research on health issues. Research has focused on factors leading to smoking acquisition in order to construct prevention programs. So far, many programs have been designed to provide people with information about the advantages and disadvantages of smoking, and to teach youngsters skills to resist pressures from peers and media (Flay, 1985; Oei \& Fae, 1987). Recent research has suggested that a considerable number of teenage smokers are well aware of the dangers and drawbacks of their tobacco use and therefore want to stop smoking (Tuakli, Smith \& Heaton, 1990). Adolescents seem to undertake quit attempts frequently, have withdrawal symptoms and might relapse more ofien than adults (Charlton, 1984; Ershler, Leventhal, Fleming \& Glynn, 1989; Pallonen, Murray, Schmid, Pirie \&c Luepker, 1990; Presti \& Lichtenstein, 1992). Few longitudinal studies, however, have investigated the factors affecting quitting behavior of young people.

During the last decades, researchers as well as prevention workers have considered smoking cessation as a complex process which involves transitions through different phases or stages in order to become an ex-smoker. Several perspectives have been proposed consisting of motivational elements, addiction and habitual factors, and actual quitting behavior (DiClemente, Prochaska, Fairhurst, Velicer, Velasquez \& Rossi, 1991; Farkas, Pierce, Zhu, Rosbrook, Gilpin \& Berry, 1996; Leventhal \& Cleary, 1980; Prochaska \& DiClemente, 1983; Prochaska, 
DiClemente \& Norcross, 1992; Sutton, 1995; Werch \& Anzalone, 1995). One of the most important outcomes from the research on stages of smoking cessation concerns the heterogeneity of the group of smokers. Prochaska and colleagues (e.g., DiClemente et al., 1991; Prochaska et al., 1983; Prochaska et al., 1992) suggest that people can move from precontemplation, contemplation and preparation to action, and then to maintenance or relapse. In terms of smoking cessation, this implies, for instance, that precontemplators are not considering quitting while preparators are planning to quit in the near future. The general assumption is that smokers in these stages will demonstrate a developmental sequence of movement to smoking cessation. Furthermore, it is assumed that moving from one stage to another is accompanied by differences in events and activities that create successful change of a problem behavior (DiClemente et al., 1991). As a result, becoming prepared to quit has been associated with becoming convinced of the pros of quitting and the cons of smoking, higher levels of self-efficacy and stronger social support during their quitting attempt(s) (De Vries \& Backbier, 1994; DiClemente et al., 1991). It is particularly of value for prevention practice to identify the factors which differentiate berween stages of cessation. An advantage is that smoking interventions can be tailored to the individual's position in a specific phase of cessation. Nevertheless, the empirical research on stages or phases in smoking cessation have been carried out in adult samples - most adolescent studies have considered quitting as a dichotomous outcome variable (see for an exception, Pallonen et al., 1990).

The vast majority of smoking cessation programs are based on research amongst adult samples. Studies of unaided smoking cessation as well as studies of more formal treatment programs have suggested that quitting is predicted by nicotine dependence factors, such as the intensity and frequency of smoking or the years of smoking, and by environmental factors, such as smoking by family members, friends or colleagues and getting social support during the quitting attempt (Breslau \& Peterson, 1996; Marlatt, Curry \& Gordon, 1988; Rose, Chassin, Presson \& Sherman, 1996; U.S. Surgeon General, 1991). Furthermore, some studies have shown that the smokers' perceptions of the pros and cons of smoking and quitting as well as their perceived efficacy to succeed when a quitting attempt is undertaken affects their quitting behavior (De Vries \& Backbier, 1994; Dijkstra, De Vries $8 x$ Bakker, 1996; Prochaska et al., 1992; Rose et al., 1996). The current study examined predictors of young people's motivation to quit using a longitudinal study on 215 regular smokers. Three categories of predictor variables were used namely smoking specific cognitions, social influences, and habitual aspects of smoking.

Atritudes towards smoking seem to be one of the central elements in the process of initiation and cessation of smoking (Ajzen, 1991; De Vries, Dijkstra \& Kuhlman, 1988). From research among adult smokers, it is known that beliefs on the social and health consequences of smoking predict quitting attempts (Eiser, Van De Pligt, Raw \& Sutton, 1985) and success (Kirscht, Janz \& Becker, 1989). An explorative study among occasional and daily adolescent smokers revealed that 
health reasons and discontentment with the addiction were related to intentions to quit (Stone \& Kristeler, 1992). The importance of smoking beliefs was also shown in a longitudinal study (Hansen, Collins, Johnson \& Graham, 1985) in which quitters were more concerned with the consequences for others and the morality of smoking - they perceived smoking as devaluing their personal worth. In addition, Rose and colleagues (1996) found that having specific motives to smoke, such as stimulation ("to perk myself up") or sensorimotor ("enjoy handling a cigarette"), are related to a less successful quitting attempt among young adults. Additionally, the self-confidence of smokers to resist external or internal pressures to continue smoking, and the ability to cope with these pressures, affect them to become and remain ex-smokers (Bandura, 1986). Some studies showed the relevance of the selfefficacy concept in relation to changes in smoking status of young people (De Vries, Backbier, Kok \& Dijkstra, 1995; Lawrence, 1989).

With regard to social influences, parental and peer use have been related to the onset of smoking by their offspring in several studies (Conrad, Flay \& Hill, 1992). Complementary to these findings, most studies found that smokers with a nonsmoking family background or with nonsmoking peers, feel more strongly motivated and supported to quit (Chassin, Presson $8 x$ Sherman, 1984; Rose et al., 1996; Skinner, Massey, Krohn \& Lauer, 1985; Stanton, Currie, Oei \& Silva, 1996; Stone \& Kristeler, 1992). Because peer groups are quite homogenous in their substance use patterns, smoking friends could stimulate others to continue smoking and discourage smoking cessation. For instance, in a qualitative study (Presti et al,, 1992) almost $20 \%$ of those who tried to quit reported that friends made it harder to stop, by offering cigarettes, harassment, and by smoking around the individual. Gordon (1986) suggested that quitting can even result in a temporary loss of friends or clique membership due to differences in smoking status. In sum, findings from longitudinal studies and qualitative work indicate that a smoking social environment makes it more difficult to quit.

Mixed results are reported on the relationships between tobacco dependence, habit formation and adolescents' ability to quit. Within a study among 18-year olds, dependent smokers were as likely as nondependent smokers to try to quit, to intend not to smoke when older, or to seek professional help for smoking-related problems (Stanton, 1995). Hansen et al. (1985) did not find support for a relation between intensity of smoking and cessation. Another study by Hansen (1983), however, showed a significant relationship between the frequency of use and the length of time a youngster remained abstinent later on. Furthermore, Breslau and co-workers (Breslau, Kilbey \& Andreski, 1994; Breslau \& Peterson, 1996) stressed that the age of initiation is a predictor of quitting. In studies among young adults, they showed that smokers who had delayed the initiation of smoking were more likely to quit than those who had begun to smoke at an earlier age. Moreover, the variety of situations in which someone smokes might be related to smoking cessation. An attempt to quit might be more difficult for those smoking in a large variety of 
(social) settings since they will be confronted with their habits on various occasions. Shiffman and Jarvis (1987; Shiffman, 1989) found that smokers trying to quit were challenged by situations in which cigarette use is commonly related to socializing with others or to relaxing and leisure activities. In general one should bear in mind that in contrast to adult smokers, young people only smoke for a limited period of time. Differences in smoking habits and tobacco dependence could not only reflect differences in people's preparations to quit but could also reflect differences in the process of initiation.

The aim of this study was to investigate the factors affecting the allocation to four stages of cessation: precontemplation, contemplation, preparation and quitting. Data for analyses were derived from a three wave longitudinal among secondary schoolchildren in the Netherlands. The main analyses concentrated on the effects of the predictor variables measured at wave two (14-15 year olds) on subjects" motivation to quit at wave three (17-18 year olds).

Our approach will contribute to other research in three ways. First, smoking cessation has been studied in many cross-sectional studies - there are only a few prospective studies which have examined the factors predicting unaided cessation by adolescents. Furthermore, some studies have tested the effects (or correlates) of specific groups of predictor variables. Only a few, however, have focused on the value of smoking-specific cognitions, social influences and habitual factors in one study (see for an exception Rose et al., 1996). Finally, the most commonly applied analytic strategy is to examine the factors distinguishing smokers from quitters. We assume that the category of smokers is quite heterogenous and making differentiations within this category according to their motivation to quit can contribute to the understanding of the quitting process.

In this study, a classification was constructed which combined the degree of motivation to quit with actual quitting behavior. The first two stages included those smokers who had not considered quitting in the future or within 6 months. The third stage involved those who were prepared to quit. The fourth group consisted of ex-smokers at the third wave. First, we investigated if our classification of smokers was related to actual smoking and quitting behavior at wave three. It was expected that precontemplators report fewer quitting attempts and are heavier smokers than contemplators and preparators. Second, univariate analyses were used to examine the factors discriminating the four groups of smokers. In general, we expect that a stronger motivation to quit (or having actually quitted) is related to having a less positive attitude towards smoking, having stronger self-efficacy expectations, experiencing more social support, and being less dependent of tobacco. Third, multivariate analyses were carried out to explore which factors affect the allocation to the different stages. We do not have hypotheses about differences or similarities in these analyses. 


\section{METHOD}

\section{Procedure}

In 1989 , the cohort study started with a selection of 65 schools differentiated to educational level and region in the Netherlands (Van Reek, Knibbe \& Van Iwaarden, 1993). Formal consent from 36 school boards was obtained. Twenty-nine secondary schools refused to participate in the study. One reason was privacy; names and addresses of subjects were needed for follow-up surveys. Furthermore, participation in other research or intervention programs was a reason for refusal. Special attention was placed on confidentiality of responses. Only the principal researcher could match the names and numbers. At the first measurement the questionnaires were administered in classrooms and returned by the subjects in a sealed envelope. 1,454 subjects of first and second grade of secondary education (mean age 12.4 years, $S D=.7$ ) completed the questionnaire.

In the second (T2) and third (T3) wave, forms were sent to subjects' homes. In 1991, parents of the subjects who did not respond, were contacted for permission to interview their child by telephone. In 1994 (subjects' mean age 17,4), subjects were directly contacted to participate in the study. Eighty-two percent of the adolescents participated in the second wave, and $82 \%$ also was enrolled in the third wave. In total, $73 \%$ of the original sample $(n=1,063)$ participated in the two follow-up surveys.

An attrition analysis was conducted to examine if those who participated in all three waves differed from those who were not enrolled in the second and/or third wave (see chapter 4). A logistic regression analysis showed no significant differences $(p>05)$ on frequency and intensity of smoking between dropouts $(n=$ 391) and respondents $(n=1,063)$.

\section{Measures}

The analyses presented in this paper are performed on data of the second and third wave with an interval of three years. All variables were included in the second wave except smoking history and smoking context which were measured at wave two and three. Additionally, questions about subjects' motivations to quit and actual quitting behavior were only incorporated in the third wave.

Stages of Change (T3). In this study, four stages of smoking cessation are presented. The classification of the smoking subjects was based on two questions about their intentions to quit smoking. The first three stages were differentiated by the itemscores on the respondents' thoughts concerning smoking when they were older, and within the next six months. This classification did not match the stage algorithms suggested by Prochaska and colleagues. As we did not wish to add new labels to this field of research, we used their labeling for the stages of smoking cessation. It should 
be clear that this article does not aim to test their stage dimensions. Notice that the difference between our classification and those employed by Prochaska et al. (e.g., DiClemente et al., 1991; Prochaska et al., 1983; Prochaska et al., 1992) consists in the time frame of the intention items. In their approach, two questions regarding seriously considering quitting within 6 months and planning to quit within 30 days were used while in our approach two questions regarding quitting when they were older and quitting within 6 months were used. Thus, in the current study, precontemplators were those smokers who did not consider quitting when they were older. Contemplators were those who did not want to smoke or were ambiguous about smoking when they were older, but did not consider quitting within the next six months. Preparators were those who were seriously considering quitting within the next six months. Quitters were those who indicated being a nonsmoker at the third wave. The classification was exclusive so that subjects could be allocated to only one stage. Subjects not providing information about either intention or behavioral aspects were omitted from analyses.

Quit Attempts (T3). At the third measurement, subjects (i.e., those who responded to the life-time use item) were asked to indicate if they had tried to quit smoking in the last three years. Subjects who responded positively to this question were asked if their attempt has been successful (for some time), the number of attempts and the duration of the last attempt. They were also asked whether they had tried to stop for 24 hours.

Smoking History (T2, T3). Subjects were asked to answer questions about age of onset, smoking in the preceding four weeks and the quantity of use. The age of onset referred to the age a subject first smoked a few puffs. Responses on smoking in the preceding four weeks were used as an indicator of the frequency of use with options: not in last four weeks (1), less than weekly (2), at least weekly (3) and daily (4). Subjects who reported smoking in the preceding four weeks were also asked to report the number of cigarettes and hand rolled cigarettes smoked per week. These two items were summed to an indicator of the quantity of tobacco use.

Smoking Context (T2, T3). Subjects who reported smoking in the preceding four weeks were asked whether they smoked in the following seven situations: school, parental living room or kitchen, own room, parties or special evenings, friends" homes, bars or discos, and on the street. Subjects responded on a dichotomous scale, and scores were subsequently summed to an 8-point scale with options ranging from no situation (0) to each situation (7).

Attitude (T2). A subset of five questions, retrieved from a 24-item attitude scale of De Vries and Kok (1986), measured the following consequences of smoking: passive smoking for others, less boredom, shortness of breath, a nice taste, and a wise act. Beliefs were measured on a 4-point scale ( 3 items) and a 7 -point scale ( 2 items). Cronbach"s alpha was .60. The sum score ranged from 5 (negative smoking attitude) to 26 (positive smoking attitude).

Self-efficacy (T2). Two items were extracted from a 9-item self-efficacy scale (De 
Vries, Dijkstra \& Kuhlman, 1988), and measured the confidence of a subject's ability to become (or stay) a nonsmoker and their confidence to refuse a cigarette when one was offered. Subjects were asked to rate their confidence on a 7 -point scale ranging from very low (1) to very high (7). The correlation between the items was .64. The sum score for self-efficacy ranged from 2 (low self-efficacy) to 14 (high self-efficacy).

Social Influences (T2). Subjects were asked if they considered their father, mother, eldest brother, eldest sister, best same-sex friend and the peer group to be smokers. Responses were: no/do not know (1) and yes (2). Peer group smoking was measured by the proportion of smokers in the peer group on a 5-point scale which ranged from no one (1) to all members smoke (5). Pressures to smoke were measured by asking if they felt that the referent others mentioned above, tried to persuade them to smoke. Response categories ranged from never (1) to very often (5).

\section{Statistical Analyses}

First, we examined whether the motivational stages of change employed in this study are actually related to quitting behavior and smoking habits at wave three. One-way analyses of variance were performed to compare the differences in smoking behavior (e.g., frequency and intensity of smoking) and quitting behavior (e.g., trying to quit, the number of attempts) between precontemplators, contemplators and preparators. If the F-test of the univariate analysis of variance appeared to be significant, the Tukey-HSD method was used for pairwise comparisons. Second, multiple univariate analyses of variance were conducted to compare the scores on the predictor variables assessed at wave two for the three smoking groups and the group of quitters at wave three. The Tukey-HSD method was employed for post hoc pairwise comparisons.

Third, multiple logistic regression analyses were performed to examine the joint effects of the predictor variables on the stages. These analyses were explorative in a sense that not an explicit model, with specifications of intercorrelations, has been tested. Basically, three analyses were carried out comparing the groups which are most closely related. Thus, the three comparisons were: precontemplators versus contemplators, contemplators versus preparators and preparators versus quitters. An additional analysis was performed to gain insight into which variables predict the allocation to the most contrasting groups (precontemplators and quitters). In order words, in what respect differ those who are absolutely immotivated (at the time of measurement) from those who are successful quitters. Thus, variables were included in analyses by a forward selection procedure. The likelihood ratio criterion was used to determine which variables should be added to the model. In order to avoid Type II errors, a probability of .10 was used for a variable to enter the equation and a probability of .15 to remove a variable from the equation (Hosmer \& Lemeshow, 1989). 


\section{RESULTS}

\section{Subjects}

Of those who participated at all three measurements, $215(20 \%)$ subjects smoked on a regular basis (i.e., had smoked in the preceding four weeks) at wave three. This sample comprised $95(44 \%)$ men and 120 women. The average age of the sample at the second measurement was $14.5(S D=.8)$. More than forty percent $(45 \%)$ were enrolled in vocational schools, $54 \%$ were enrolled in high schools and $1 \%$ were not enrolled in secondary education at wave two. Nineteen percent reported that their father was Roman Carholic, 28\% Protestant, 3\% Islamic, 4\% followed another religion, and $46 \%$ were not religious. Most subjects (78\%) lived with both parents, $18 \%$ with one parent, and $4 \%$ had left the parental home. Three years later, 68 subjects were classified as precontemplators, 50 subjects as contemplators and 40 subjects as preparators. Twenty-five T2-smokers did not smoke at the third wave. 32 subjects could not be allocated to one of the stages of change due to missing data. ${ }^{2}$

Table 9.1. Means and Differences in Quitting Behavior and Habitual Variables between Three Smoking Groups at the Third Wave; Cross-sectional Analyses

\begin{tabular}{|c|c|c|c|c|c|}
\hline T3 wariables & $\begin{array}{c}\mathrm{Pc} \\
(68)\end{array}$ & $\begin{array}{c}\mathrm{C} \\
(50)\end{array}$ & $\begin{array}{c}\mathrm{Pa} \\
(40)\end{array}$ & F-test & Tukey-HSD" \\
\hline \multicolumn{6}{|l|}{ Quatting } \\
\hline Tried to quit (\%) & 43 & 60 & 80 & $7.87^{* * 1+3}$ & $\mathrm{Pc}<\mathrm{Pa}$ \\
\hline Successful attempt (\%) & 40 & 62 & 68 & $5.13^{\text {将 }}$ & $\mathrm{Pc}<\mathrm{C}, \mathrm{Pa}$ \\
\hline 24 hours attempt (\%) & 55 & 62 & 79 & $3.17^{\text {25.44 }}$ & $\mathrm{Pc}<\mathrm{Pa}$ \\
\hline No. attempts & 1.75 & 1.26 & 1.88 & $.63 \mathrm{~ns}$ & - \\
\hline \multicolumn{6}{|l|}{ Smoking Bebavior } \\
\hline No. cigarettes & 81.41 & 72.60 & 53.35 & $5.41^{\text {* B }}$ & $\mathrm{Pa}<\mathrm{Pc}$ \\
\hline Frequency & 3.82 & 3.88 & 3.55 & $4.92^{\text {年皮 }}$ & $\mathrm{Pa}<\mathrm{Pc}, \mathrm{C}$ \\
\hline Age of onset & 12.15 & 11.96 & 12.60 & $1.02 \mathrm{~ns}$ & - \\
\hline Total of 7 situations & 5.62 & 5.52 & 4.83 & $3.79^{24}$ & $\mathrm{~Pa}<\mathrm{Pc}$ \\
\hline
\end{tabular}

Note. Pc Precontemplators, $\mathrm{C}=$ Contemplators, $\mathrm{Pa}=$ Preparators. ns $=$ not significant $(p>.05),{ }^{*} p<.05, p<.01, \vec{p}<.001 .{ }^{\circ} \mathrm{Pc}<\mathrm{Pa}$ implies that the mean of $\mathrm{Pc}$ differs significantly from the mean of Pa. One-way analyses with Tukey-HSD contrasts.

Differences in Quitting Behavior and Smoking Behavior between Precontemplators, Contemplators and Preparators

Prelliminary analyses were used to verify whether subjects (i.e., all four smoking groups) varied on some socio-demographic variables. Since differences in gender, education, origin and family structure were not significant $(p>10)$, no separate 
analyses for these items were conducted.

The classification of smoking adolescents into three groups based on differences in their motivation to quit was related to actual quitting variables and smoking behavior (Table 9.1). In this way, we verified whether our classification not only reflected differences in intentions but was also associated with other possible indicators of the quitting process. Prepared smokers were more likely to have undertaken a quit attempt or a 24 -hours quit attempt. They were also more successful in accomplishing a nonsmoking period. For instance, $68 \%$ of the preparators reported a successful quit attempt compared to $62 \%$ of the contemplators and $40 \%$ of the precontemplators. Furthermore, preparators smoked less cigarettes $(M=53.35)$ than precontemplators $(M=72.6)$. Precontemplators and contemplators $(M=81.41)$, however, did not differ significantly. Moreover, data on the frequency of smoking revealed that preparators smoked less often that the other two smoking groups. For example, $85 \%$ of the precontemplators and $92 \%$ of the contemplators were daily smokers compared to $68 \%$ of the preparators. No significant differences were found for the age of onset. The duration of smoking habit was not related to the stages of smoking cessation. The groups differed in the number of settings in which they used cigarettes. Additional analyses on each of the seven situations showed that within the preparating group, less respondents smoked at friends' homes (compared with precontemplators) and on the street (compared with precontemplators and contemplators).

\section{Differences in Antecedents across Four Stages of Smoking Cessation}

Differences in attitude and self-efficacy were associated with the allocation to the stages of change in the hypothesized direction. Those smokers holding positive attitudes to smoking and with lower levels of confidence in cessation efforts were more likely to be precontemplators than preparing to quit or actually having quit three years later (Table 9.2). In particular, differences were revealed between the most extreme stages of change. Less differences were found between the more closest groups of smokers. For example, precontemplators and contemplators dit not differ in smoking-related cognitions three years earlier. Furthermore, neither smoking by a best same sex friend, the peer group or family members nor the perceived pressure to smoke by significant others were related to the stages. Habitual factors appeared to be important regarding the stages of smoking. Differences in frequency and intensity of smoking were related to the stages - those who smoked many cigarettes per week at the second wave were less likely to be motivated to quit three years later. Additionally, those subjects who were smoking occasionally at wave two were much more likely to quit or to be prepared to quit three years later. Furthermore, those who smoked in various situations were less likely to be motivated to quit. Separate one-way analyses of variance showed that those who smoked in social situations such as parties and special evenings, and bars 
and discos were more likely to be precontemplators or contemplators than quitters three years later. Differences in smoking at either their own home, their friends' homes, or on the street did not affect young people's motivation to change later on. Although it was expected that the length of time somebody smokes was related to the motivation to quit, we found no effect of age of onset on allocation to the stages.

Table 9.2. Univariate Analyses of Differences in Predictor Variables for the Four Smoking Groups at the Third Wave; Longitudinal Analyses

\begin{tabular}{|c|c|c|c|c|c|c|}
\hline T2 variables & $\begin{array}{l}\mathrm{Pc} \\
(68)\end{array}$ & $\begin{array}{c}\mathrm{C} \\
(50)\end{array}$ & $\begin{array}{c}\mathrm{Pa} \\
(40) \\
\end{array}$ & $\begin{array}{c}Q \\
(25)\end{array}$ & F-test & Tukey-HSD \\
\hline \multicolumn{7}{|l|}{ Cognitions } \\
\hline Attitude & 16.84 & 16.13 & 15.44 & 14.42 & 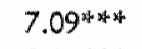 & $\mathrm{Pc}>\mathrm{Pa}, \mathrm{Q} ; \mathrm{C}>\mathrm{Q}$ \\
\hline Self-efficacy & 7.42 & 7.85 & 9.44 & 11.22 & $8.211 \%$ & $\mathrm{P}_{\mathrm{c}}<\mathrm{Pa}, \mathrm{Q} ; \mathrm{C}<\mathrm{Q}$ \\
\hline \multicolumn{7}{|l|}{ Social infinences } \\
\hline \multicolumn{7}{|l|}{ Smoking by } \\
\hline Family members & 5.75 & 5.32 & 5.67 & 5.28 & $3.16^{*}$ & - \\
\hline Best same sex friend & 1.79 & 1.82 & 1.65 & 1.80 & $1.44 \mathrm{~ns}$ & - \\
\hline Peer group & 3.73 & 3.60 & 3.33 & 3.50 & $1.45 \mathrm{~ns}$ & - \\
\hline \multicolumn{7}{|l|}{ Pressure to smoke by } \\
\hline Family members & 1.10 & 1.04 & 1.09 & 1.04 & $1.15 \mathrm{~ns}$ & - \\
\hline Best same sex friend & 1.63 & 1.60 & 1.55 & 1.80 & $.32 \mathrm{~ns}$ & - \\
\hline $\begin{array}{l}\text { Peer group } \\
\text { Smoking behavior }\end{array}$ & 1.75 & 1.82 & 1.93 & 1.88 & $.28 \mathrm{~ns}$ & - \\
\hline No. cigarettes & 29.37 & 25.04 & 21.65 & 8.32 & $4.27^{n+4}$ & $Q<P c, C$ \\
\hline Frequency & 2.44 & 2.46 & 2.00 & 1.48 & $12.60^{* * 44}$ & $\begin{array}{l}\mathrm{Q}<\mathrm{PC}, \mathrm{C}, \mathrm{Pa}_{3} \\
\mathrm{~Pa}_{\mathrm{a}}<\mathrm{P}_{\mathrm{c}} \mathrm{C}\end{array}$ \\
\hline Age of onset & 11.68 & 11.23 & 12.28 & 12.25 & $2.53 \mathrm{~ns}$ & - \\
\hline Total of 7 situations & 4.41 & 3.94 & 3.70 & 2.56 & $5.24^{* * * t}$ & $\mathrm{Q}<\mathrm{Pc}, \mathrm{C}$ \\
\hline
\end{tabular}

\section{Multivariate Relations between Predictor Variables and Motivation to Change}

Logistic regression analyses were conducted to investigate which variables predicted the allocation to the stages multivariately. Three analyses were carried out to compare the groups which are most closely related (Table 9.3). ${ }^{3}$ Precontemplators and contemplators did not differ on smoking behavior or smoking cognitions three years earlier. In contrast, those who smoked more frequently and started to smoke at an earlier age were more likely to be contemplator than preparator three years later. Furthermore, smoking cognitions did not distinguish those who were 
prepared to quit from those who had actually quit. Only the intensity of cigarette smoking affected the allocation to one of these groups. In sum, it appeared that in all three analyses the scores on smoking attitudes and self-efficacy did not predict the allocation to these groups. Particularly differences in smoking habits (or dependency) affected the motivation to quit. However, between the two groups who not motivated to quit in the near future (precontemplators and contemplators), no differences were found on the set of variables employed in this study. Notice that the variables only predicted a small amount of variance in the dependent variable. The explained variance was in the second analysis, comparing contemplators with preparators, six percent and in the third analysis, comparing preparators and quitters, eleven percent.

Table 9.3. Logistic Regression Analyses of Predictor Variables on Adolescents" Motivation to Quiv

OR $(95 \% \mathrm{Cl})^{2}$

Precontemplators versus Contemplators

No predictor variables included

Contempators versas Preparators

Frequency of smoking

Age of onset

$.56 \quad(.31-1.01) \quad<.05$

$1.37(1.02-1.83) \quad<.05$

Preparators versus Quitters

No. cigarettes

$.96 \quad(.93-1.00)$

$<.05$

"Variables were included in analyses by a forward selection procedure and the likelihood ratio criterion was used to determine which variables should be added to the model. A probability of .10 was used for a variable to enter the equation and a probability of 15 to remove a variable from the equation. "This implies that no wariable contributed to the prediction after the variable frequency of smoking and age of onset entered the equation.

An additional analysis was carried out which compared the most contrasting groups differing most (precontemplators - quitters). ${ }^{4}$ This analysis displayed that those who are immotivated to quit differ from the successful quitters in their scores on the smoking cognitions. Those with a negative attitude towards smoking (OR = $.72,95 \% \mathrm{CI}=.56-93, p<.01)$ and with high self-efficacy expectations $(\mathrm{OR}=1.35$, $95 \% \mathrm{CI}=1.11=1.64, p<.001)$ were more likely to be quitters later on. Indicators of smoking habits did not discriminate precontemplators from quitters in this multivariate analysis. The explained variance was $26 \%$.

\section{DISCUSSION}

The present study explored the predictors of young smokers" motivation to quit using a set of variables consisting of smoking-related cognitions, social influences 
and habitual factors. Differences in cognitions were related to the stages three years later. Within the group of regular smokers, those who perceived the consequences of smoking as positive and disregarded the negative aspects, and those with lower self-efficacy scores were less likely to be motivated to quit later on. Within a longitudinal study, Rose et al. (1996) also found that motives to smoke (and to quit) are related to quitting among those who attempted to quit. Although they differentiated smokers on their quitting behavior and not on their motivation to quit, both studies display the long term ( 3 years in our study and 7 years in Rose et al.) effects of smoking cognitions on quitting behavior. The multivariate analyses in the current study, however, showed that these cognitions distinguished those who were not motivated to quit from those who actually quit but that they did not distinguish between all smoking groups (for instance, preparators and quitters). Some cross-sectional studies among adults also reported that differences in cognitions (such as attitude and self-efficacy) were evident between the extremes of the continuum but not between all stages of cessation (De Vries \& Backbier, 1994; Dijkstra, et al., 1996).

Smoking by peers, a best same sex friend or family members were not related to smoking cessation in both univariate and multivariate analyses. These findings, which were in contrast with those of other studies (Chassin et al., 1984; Chassin, Presson, Rose \& Sherman, 1996; Skinner et al., 1985; Stone \& Kristeller, 1992), might be explained by the extended interval between the waves. Perhaps parents have some impact on their offsprings' motivation to quit in the short term, but have a limited effect in the long run (i.e., when their children have already smoked for some time). Another explanation comes from Flay et al. (1994) who suggested that social influences might have an indirect effect on smoking. The perception of smoking by others could affect adolescents' attitudes and self-efficacy which could subsequently affect their intentions to smoke. However, the findings of the logistic regression analyses presented in this study do not make this suggestion very likely. Another explanation is that smokers might not want to be confronted with the explicit norms and pressures of nonsmokers or quitters, and seek friends with similar habits and opinions about cessation (Engels, Knibbe, Drop \& De Haan, 1997; Ennett \& Bauman, 1994; Gordon, 1986). In other words, the 'die hards' cluster together into homogenous groups. This could explain the absence of significant effects of peer smoking on adolescents' motivation to quit.

The univariate analyses clearly showed that the intensity and frequency of smoking affected the motivation to quit three years later. Those who smoked daily and used many cigarettes per week were less likely to be motivated to quit, or to have quit three years later. These findings coincide with the work of Stanton (1995) on tobacco dependence and quitting behavior. Our data were also indicative of the relevance of the context of smoking when looking at the process of cessation. Particularly smoking in social settings, such as public drinking places and parties determined whether subjects still smoked or quit. Public drinking places and parties 
could be 'high risk situations' (Shiffman \& Jarvis, 1987) which are linked to the maintenance of smoking.

The major difference between the univariate and multivariate analyses remains in the absence of the smoking cognitions in the equations. We stress that these analyses are used for explorative purposes. The strong intercorrelations between items concerning smoking behavior and cognitions, such as attitudes and self-efficacy, as is shown in many other (cross-sectional) surveys, makes it difficult to draw definite conclusions from these analyses. Nonetheless, the univariate analyses clearly show that both smoking cognitions and tobacco dependence are precursors of young people's motivation to quit.

In the present study, antecedents of quitting were examined using a classification which differentiated smokers on their motivation to quit. Although these smoking groups were not comparable to those suggested by Prochaska and colleagues, the utility of our classification was shown by the actual quitting and smoking behavior across the three smoking groups. Differences in quit attempts were found across the smoking groups in the expected direction. The three smoking groups also varied on the quantity and frequency of tobacco use. For example, the group precontemplators consisted of many more daily smokers than the group preparators. Further research should, however, reveal whether the classification used in this study reflects all the aspects of the process of cessation. For instance, information about the future allocation of the smoking subjects is necessary to examine the external validity of this classification. We do not know if our classification contains different groups or categories of smokers, or incorporates smokers in different stages of cessation. In addition, it should be mentioned that our analyses were based on a group of regular smokers. It is possible that in the early stages of smoking (Leventhal \& Cleary, 1980) - initiation and experimentation people weigh up the pros and cons and, eventually, decide to quit or to continue smoking. There could be a critical point where continued smoking goes along with a declining interest in quitting. This might also explain the small number of quitters in this study. All in all, our understanding of the quitting process would perhaps be enhanced if, at the same time, the process of initiation were taken into consideration. For adolescent samples, the stages of initiation and cessation might operate parallel to each other (see Werch \& Anzalone, 1995). It would be worth while to examine in more detail how young people progress through stages of onset and cessation, and whether similar factors affect them.

Our study is subject to some limitations. First, the incidence of quitting was rather low; only 25 subjects reported having quit between the wraves. This implies that when studies concentrate on the early grades, with a low prevalence of smoking and quitting, large numbers of respondents are required to yield informative conclusions. Furthermore, the current study is explorative in a sense that it provides insight into the precursors of smoking cessation using some general measures of cognitions. A specific self-efficacy scale would identify the situations and conditions 
in which individual smokers find it hard not to smoke and lack sufficient coping strategies (Lawrence, 1989). In addition, an assessment of the pros and cons of botb smoking and quitting might provide precise information on which beliefs affect adolescents" motivation to quit.

Another concern is the rather low internal consistency estimate for the attitude scale $(\alpha=60)$. We assume that one of the reasons for this estimate remains in the heterogeneity of the content of the five items. Although previous research (De Vries \& Kok, 1986) have shown that these beliefs are associated with youngsters' smoking behavior, distracting singular items from different domains (and subscales) might not result in a high consistent scale. It is recommended to measure different attitudinal domains with separate scales in future research.

Although the smoking status of significant others provides normative information about their ideas on smoking, other measures of social influence could be considered (De Vries et al., 1995). Support from parents or peers might help youngsters with their attempts to quit. Research on adult samples has already shown that the perception of social support is positively associated with smokers' attempts to quit (Cohen \& Lichtenstein, 1990). In addition, an assessment of social encouragement to smoke cigarettes might be more complicated than simply asking youngsters whether they experienced pressure from significant others (Friedman, Lichtenstein \& Biglan, 1985).

Self-reports were used to obtain an indication of subjects' actual smoking behavior. In this study, a biochemical test of smoking (e.g., saliva cotinine test, Abrams, Follick, Biener, Carey \& Hitti, 1987) was not included. However, special attention was paid to assure confidentiality in order to obtain accurate reports of subjects' own use. According to Botvin and Botvin (1992), this is one of the ways of obtaining reliable and valid responses. All in all, we do not think that our findings are significantly distorted with respect to subjects' current smoking status.

School-based prevention programs have overlooked the issue of smoking cessation among teenagers. Only a few cessation programs are constructed for adolescents which are mostly based on adult approaches, or on the determinants of onset. Although this study is not conclusive about the determinants of quitting, some recommendations for secondary prevention can be formulated. In our study, it appeared that many young smokers were not motivated to quit (almost thirty percent thought that they would not stop when older). Since these findings are based on a sample of regular smokers, it might be more effective to approach those who are still not smoking on a regular basis. Other studies also stress that 'there may be a point in the sequence beyond which quitting is far less likely to be successful' (Erschler et al., 1989 p. 376, Glynn, Leventhal \& Hirschman, 1985). One of the interesting findings of this study is that modelling and social pressure by friends as well as nuclear family did not affected young people's motivation to change. Although one can debate about the interpretation of these results, interventions primarily focussing on teaching youngsters skills to resist explicit or 
implicit peer pressure to continue smoking, as is done in many primary prevention programs, are expected to have limited effects. In contrast, our research showed that an approach should involve modification of smoking cognitions. We stress that making people aware of their atritudes towards smoking (and quitting) and their personal barriers regarding quitting might enhance the motivation to change their habits. For instance, a program in which different groups of smokers are involved might help especially immotivated people to become aware of their opinions (see group discussion approach, Charlton, 1984). However, the strong impact of smoking habits on adolescents' quitting behavior suggests that programs should incorporate methods which tackle the psychological and physiological dependence on nicotine among young people. Research is required on development, implementation and evaluation of such programs (Burton, 1994; Prokhorov, Pallonen, Fava, Ding \& Niaura, 1996).

In conclusion, this study clearly shows that smoking cessation of young people should be put on the research agenda. In a period of three years, only a small percentage of the regular smokers have quitted $(12 \%)$. Our findings also demonstrate that many young smokers are absolutely not motivated to quit. In terms of predictors of people's motivation to quit, it appeared that subjects' opinions about smoking or their efficacy to resist temptations to smoke affect people's intentions and abilities to quit. But tobacco dependence and habit formation are important factors in the process of smoking cessation, not only for adults who smoke for many years but also for teenagers. Furthermore, the intriguing non-significant relationships between peer factors and quitting in this study also deserves attention in future research. Finally, we assume that models which consider smoking as a process involving different stages of behavioral and cognitive change can help us to display the determinants of smoking cessation of adolescents.

\section{NOTES}

\section{${ }^{1}$ Based on:}

Engels, R.C.M.E., Knibbe, R.A., De Vries, H., \& Drop, M.J. (1998). Antecedents of smoking cessation among adolescents: Who is motivated to change? Preventive Medicine, 27, 348-357.

2 We did not gather information from 32 subjects about their smoking intentions. So these subjects could not be allocated to one of the stages of change. An additional attrition analysis was conducted to verify if this group would turn out to be selective. However, no significant differences $(p>.10)$ were found for the intensity and frequency of smoking between these subjects $(n=32)$ and those who were classified $(n=183)$.

${ }^{3}$ Only the cognitions and the behavioral variables were included in the logistic regression analyses. The social influence variables were omitted because (a) there were no differences between the groups at a univariate level and (b) the (non-significant) trends were not linear. Concerning the latter point, it is rather puzzling that contemplators reported to have less 


\section{Chapter 9}

smoking family members compared to both precontemplators and preparators.

"One of the implicit assumptions of our classification is that the endpoint of the motivational scale is quitting itself. So it is expected that quitters had the strongest motivation to quit at an earlier wave. Although we did not measure their motivation to quit, as described in the Method section, at the second wave, we did ask a general question on their intentions to quit. Results showed a linear trend indicating that those with the lowest intention were precontemplators three years later, and that those with the highest intention were quitters three years later. 


\section{INCONSISTENCIES IN ADOLESCENTS' SELF-REPORTS OF INITIATION OF ALCOHOL AND TOBACCO USE ${ }^{1}$}

Studies concerned with predictors of initiation and maintenance of substance use by young people rely heavily on self-reports. Most empirical literature does not address problems of errors in measurements, or assumes that "... deficiencies are not severe enough to impeach the main findings' (Kozlowski \& Heatherton, 1990, p. 54). However, behavioral variables, such as actual and prior smoking, drinking or drug habits, might be susceptible to specific assessment errors. Although investigators in the field of alcohol use (Akers, Massey, Clarke \& Lauer, 1983; Needle, McCubbin, Lorence \& Hochhauser, 1983), drug use (Barnea, Rahav \& Teichman, 1987; O'Malley, Bachman \& Johnson, 1984), and cigarette smoking (Evans, Hanson \& Mittelmark, 1977; Martin \& Newman, 1988) have discussed the validity and reliability of measures of consumption in self-reports by adolescents, less attention has been paid to the accuracy of self-reports about the precise time-point of first substance use.

One way to study errors in self-reports refers to consistency over time. A commonly applied method to measure the coherence of responses over a certain time-period is the test-retest reliability. According to Czarnecki, Russell, Cooper $8 x$ Salter (1990) test-retest procedures consist of repeated interviews conducting the same or parallel test-forms. Another approach refers to a reinterview where subjects are asked to provide information about their earlier responses (e.g. recall history of alcohol use).

In general, correlational analyses are used to determine the reliability of measurements of variables which are assumed to be stable over time. Substance use by adolescents is in principle not stable but varies throughout adolescence. 
However, if these changes are gradual, correlational parameters may overestimate the reliability of responses longitudinally. Therefore, additional correspondence methods should be employed to evaluate reliability in self-reports. Another limitation of correlational analysis might be the lack of adjustment for the prevalence of non-use (Maisto, McKay \& Connors, 1990; Single, Kandel \& Johnson, 1975). Substances that are infrequently used by adolescents, such as marijuana, will show the highest internal consistency (Needle et al., 1983). In these cases, exclusion of non-users will lead to lower percentages of agreement or correlates of responses over time. Therefore, correlational parameters will systematically be high for behavior which is seldom exhibited and reported. This study will examine the reliability of the assessment of age at first tobacco and alcohol use by adolescents who reported having drunk alcohol or smoked cigarettes at the first measurement. Correspondence methods were used to determine the accuracy of estimates by comparing estimates provided at three waves.

\section{Age at First Use}

In several studies on drug use, the age of initial experimentation is assumed to be a determinant of substance use or abuse at later ages (Flemming, Kellam $\&$ Brown, 1982; Mills \& Noyes, 1984; Newcomb \& Bentler, 1986). Smoking a few puffs or trying a sip of beer or wine in early adolescence is considered to be the starting point of a career of use. Moreover, age of onset is presumed to be one of the indicators that reflects the level of addiction to cigarette smoking (Fava, Velicer \& Prochaska, 1995). However, only a few studies have focused on the reliability of assessment of "age of onset". In a longitudinal study among 6-8-grade students, Bailey, Flewelling and Rachal (1992) examined inconsistencies in self-reports of age of initiation of drinking and marijuana use. When only the users were taken into account $32.9 \%$ of the subjects made inaccurate estimations of their age at first alcohol use after a 1 year follow-up. For marijuana use, an identical pattern was found ( $26.9 \%$ made estimation errors). Thus, youngsters who were using substances suffered from 'biases of retrospective recall' (see also Krall, Valadian, Dwyer \& Gardner, 1989; Todds \& Laws, 1959).

Although questions have been raised about the reliability of self-reports with respect to the age of initiation, assumptions about the magnitude of inconsistencies are still unaddressed. Studies with short intervals between waves are not suitable for exploring the effects of inconsistencies over a longer period of time, and do not allow conclusions to be drawn about possible trends in inconsistent estimations. In her review, Midanilk (1988) stated that people were likely to alter their cognitions about earlier use. This might result in distortions about the real time-point of onset after substance use has progressed. We assume that there is an upward trend towards a higher age of onset. On the other hand, differences in responses might be distributed around zero, with no recognizable pattern at all. 
This study aims to explore variations in self-reports about age of onset measures. Furthermore, directions of feasible changes in reported age of first use are examined. Data for analyses were derived from a five-year prospective study: estimates of age of first use were compared for three waves with intervals of two and three years, respectively. Variations in reported age of onset across waves would, of course, influence the value of age of onset as a predictor of later use. The first assessment of age of onset is assumed to be the most reliable indicator available. The accuracy of predictions is examined by comparing estimations of age of first use at first, second and third wave with actual smoking and drinking behavior at the third wave. These analyses lead to conclusions about whether, irrespective of errors in estimates of onset, correlations with actual smoking and drinking are stable and significant. If these correlations are stable but not significant, there is no predictive value. If the self-reports of age of first use are inconsistent, and only later assessments of age of first use are significant, the predictive value is doubtful. If only the estimate at the baseline measurement is significant, this concept has predictive value. However, if estimations are inconsistent, the time of measurement of age of onset must be very close to the actual age of first use to consider the predictive value.

\section{METHOD}

\section{Procedure and Sample Characteristics}

In 1989 a three-wave longitudinal study was started with a sample of 1,454 students from first and second grade of secondary education in the Netherlands (Van Reek, Knibbe \& Iwaarden, 1993). A selection of 65 schools differentiated by educational level and region was approached for participation in the study. From the vocational schools $(n=17)$ three schools refused to participate. The refusal rate for the high schools $(n=48)$ was higher. We did not obtain formal consent from twenty-six school boards. One reason was privacy: names and addresses of subjects were needed for follow-up surveys. Furthermore, participation in other research or intervention programs was a reason for refusal. Finally, a sample of 36 schools participated. Speciall attention was placed on confidentiality of responses. Only the principal researcher could match the names and numbers. Subjects were also told that only the researchers had access to the data. Parents, teachers or peers could not obtain any information about subjects' responses.

At the first measurement the questionnaires were administered in the classrooms in the presence of a teacher, and the questionnaires were returned in a sealed envelope. No explicit refusals were recorded; non-response was exclusively due to absence (e.g., sickness) at the day of assessment. In the autumn of 1991 and 1994 forms were sent to subjects" homes. More than $76 \%$ of the adolescents returned the questionnaire in the first follow-up, and $72 \%$ in the second follow-up. 
Subjects who did not respond to mailed reminders were approached by phone to complete an interview. During the telephone interview, however, no information was obtained about subjects' age of first substance use. Combining the responses of the questionnaires and the telephone interviews, $1,192(82 \%)$ and $1.187(82 \%)$ subjects particpated in 1991 and 1994 , respectively. In conclusion, a total of 1063 adolescents $(73 \%)$ were enrolled in all three waves. Attrition analyses are addressed elsewhere (see Chapter 4). The sample participating at all three waves consisted of 520 girls $(49 \%)$ and 543 boys $(51 \%)$. At the first wave the mean age was 12.4 (SD = .69). In 1989 one-third of the students were enrolled in vocational school, and 64\% in high school. Most youngsters ( $85 \%$ ) lived with two parents (and no stepparents). Ninety-three percent had a father or mother of Dutch origin.

\section{Measures}

The self-report questionnaire included measurements of potential predictors of cigarette smoking and alcohol use, and an extensive list of items on history of use and actual use. Two questions were of importance for this paper. First, subjects were asked to answer the question: 'Have you ever smoked a cigarette, even a fere puffs?'. Subjects who responded positively to this particular item were asked: 'How oll were you, when you first smoked a few puffs?". A 6-point scale was constructed to assess smoking behavior with the categories: 1 'have never smoked', 2 'have experimented with smoking, but have not smoked in the last 3 months", 3 "have experimented with smoking, but have not smoked in the last 4 weeks", 4 'have smolked occasionally, less than weekly', 5 'smoke at least once per week' and 6 'smoke daily". A quantitative measurement was obtained by adding numbers of manufactured cigarettes and hand-rolled cigarettes smoked in the last seven days. These latter questions were filled out by subjects who indicated having smoked in the preceding four weeks.

An analogous strategy was used for alcohol use. Subjects were asked to report their life-time drinking with the question: 'Have you ever drunk alcohol, even a sip?'. Responses were supplied for three categories: beer, distilled spirits and other beverages. Age of onset was subsequently assessed with the question: "How old were you when you first tried an alcoholic beverage?'. In addition, a classification was constructed to assess drinking: 1 'total abstainer', 2 'experimental drinker, has not consumed alcohol in the last 6 months', 3 'experimental drinker, has not consumed alcohol in the last 4 weeks", 4 "occasional drinker, has consumed alcohol in the last four weeks" and 5 "regular drinker, consumes alcohol at least once a week'. In addition, subjects who had consumed alcohol in the preceding four weeks were asked the number of glasses they had drunk during weekdays and the weekend of the preceding week. Those two items were added to serve as a quantitative measurement of intensity of alcohol use. 
Subsequent analyses were performed on cleaned data which had been inspected for sanges and errors. For example, data files were examined for patterns where, at a certain time point, the reported age of onset was higher than the respondent's age. Illogical outcomes were apparent in a few cases, and were substituted with missing values. One of the aims of this study was to examine the accuracy of responses to age of onset measures over an extended period of time. Therefore, the analyses were restricted to subjects who provided information about their age of initial substance use at the first measurement. Thus, subsequent analyses were conducted with 338 adolescents for smoking, and 523 adolescents for alcohol use. For these selected categories, mean ages of initiation of use at the three time-points were calculated to determine the direction of discrepancies in estimations at the aggregate level.

At the individual level, correspondence of estimates was examined by comparing self-reports about age of first use estimates reported at the first and third wave. To demonstrate the magnitude of inconsistencies in estimations over time, the two waves with the largest time-interval were chosen for comparison. In order to investigate whether inconsistencies in 'age of onset' measures affected associations with behavioral outcome measures, correlational (Pearson R) analyses were computed between reported age of onset at each (3) wave and frequency and intensity of cigarette smoking and alcohol use at the last wave.

In this article, we defined underestimation as the tendency to report a higher age of onset at the second or third wave compared to an earlier wave. Overestimation is the tendency to report an earlier age of onset at a later wave. It. must be stated that although some subjects might provide consistent estimates over the three waves, this does not imply that their reported age of onset is also the actual age of onset. Youngsters could have given an incorrect answer at the first wave. Finally, errors may be owing to denial of earlier use. For example, if subjects reported that they had started drinking at wave one, and five years later they indicated being a life-time abstainer, reports would be inconsistent. The extent of later denial of use will be addressed in this paper.

\section{RESULTS}

\section{Variations in Reported Age of First Use at the Three Waves}

Figure 10.1 depicts the mean age of alcohol and cigarette use for subjects who provided information about their age of initial use at wave one. Reported age of first drinking increased during the five year period of the study. At the first measurement adolescents reported an average age of 9.8 years $(S D=2.18)$. The mean age increased to $12.02(S D=2.32)$ at the second wave, and $13.53(S D=2.47)$ at the last wave. Paired $i$-tests were significant $(p<.05)$ for all three comparisons. 
Findings for the first use of cigarettes showed an identical pattern. In 1989, the mean age was 10.52 years $(S D=1.89)$. Two years later subjects indicated an average age of $11.63(S D=1.99)$ while an average age of $12.42(S D=2.18)$ was reported at the last wave. All estimates differed significantly at the .05 level. Generally, these results show that young people's reports of age of first smoking and drinking increases with age. Differences were not randomly divided: adolescents tended to underestimate their years of drinking and smoking.

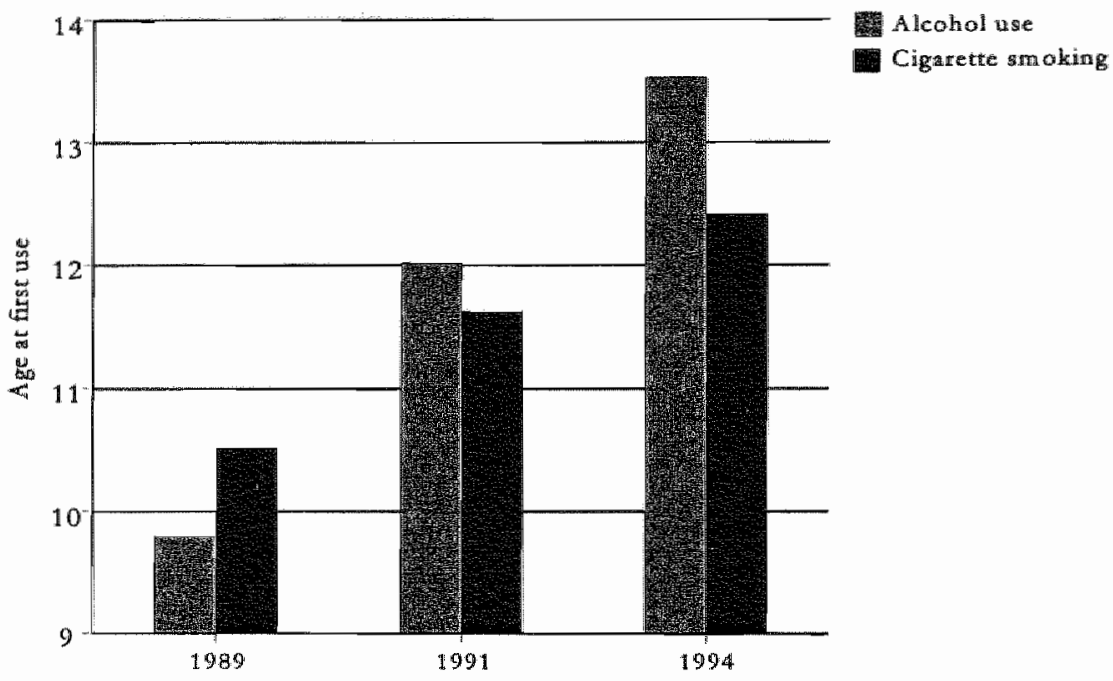

Figure 10.1. Means of Age at First Use of Alcohol and Cigarettes at the Three Waves (only for those who reported life-time use at the first wave).

\section{Magnitude of Errors in Estimations}

The data presented above show the gradual increase in reported age of first use during the three waves of this study. In Table 10.1 the reported age of onset at the first measurement and last measurement are compared in more detail. Examination of results concerning measures of age at first alcohol use shows that barely one out of twenty adolescents supplied an identical response after five years. Underestimation of age at first use by one to three years was identified for 6.1 percent. The majority of youngsters $(89 \%)$ reported an older age at wave three. Adolescents were most likely to err by five or more years $(35.6 \%)$ rather than to err by one or two year $(21.3 \%)$ or three or four years $(32.1 \%)$. Young people did not deny their initial alcohol use later on. A small percentage (13.6) supplied consistent 
estimates about their age of first cigarette use. Overestimations of smoking years ranged from one to three years. A total of $7.7 \%$ reported this type of estimation errors. Most adolescents were likely to report a higher age at the last measurement. Differences in ages ranged from one to more than five years. Nearly a third of the sample erred by one or two years, and $19.6 \%$ erred by three or four years. Discrepancies of five (or more) years were displayed for $10 \%$ of the sample. Furthermore, $14.2 \%$ of the subjects denied their earlier cigarette use at the last measurement.

A comparison of the magnitude of estimation errors for both substances displayed remarkable differences. Adolescents were more able to make consistent estimations about initiation of smoking than of drinking. Additionally, a small number of subjects overestimated the years of their substance use. Underestimation of smoking $(61.7 \%)$ and drinking $(89 \%)$ was, however, quite common in this subsample. Findings of this study suggest that subjects were more likely to report older (differences of more than three years) ages for alcohol use than cigarette use. With regard to smoking, 19.6 percent erred by 4 or more years while 54.7 percent made these estimation errors for drinking. None of the subjects denied their earlier drinking, while one out of seven adolescents denied earlier smoking a few years later.

Table 10.1. Comparisons of Reported Age at First Use of Alcohol and Cigarettes at Wave One and Three

\begin{tabular}{lccccc}
\hline & \multicolumn{2}{c}{ First alcohol use } & & \multicolumn{2}{c}{ First cigarette use } \\
\cline { 2 - 3 } \cline { 5 - 6 } & $\mathrm{N}$ & $\%$ & & $\mathrm{~N}$ & $\%$ \\
\hline Consistent estimates & 24 & 4.6 & & 46 & 13.6 \\
Inconsistent estimates & 10 & 1.9 & & 7 & 2.1 \\
$-3^{3}$ & 11 & 2.1 & & 5 & 1.5 \\
-2 & 11 & 2.1 & & 14 & 4.1 \\
-1 & & & & \\
& 37 & 7.1 & & 53 & 15.7 \\
+1 & 74 & 14.2 & & 55 & 16.3 \\
+2 & 68 & 13.0 & & 34 & 10.1 \\
+3 & 100 & 19.1 & & 32 & 9.5 \\
+4 & 186 & 35.6 & & 34 & 10.1 \\
25 & & & & 48 & 14.2 \\
& & & 10 & 3.0 \\
\hline
\end{tabular}

Note. Only those subjects are included who provided information about their age of initial use at the first measurement. The samples for alcohol and cigarette use were 523 and 338 , respectively. ${ }^{2}$ the reported age of first use at the third wave is three years earlier than the reported age at the baseline measurement. 
Table 10.2 depicts correlation coefficients for age of onset measures at each wave with actual use at the last measurement. Positive coefficients signify that reported initial use at an early age is associated with a higher frequency or intensity of smoking and drinking. The age of initiation of smoking, reported at the baseline measurement, was not significantly related to the frequency or intensity of smoking at wave three. Two years later, the age at first smoking was modestly correlated with the frequency (.15) and intensity (.09) of smoking. The highest associations were found for the reported age of onset and cigarette use at the last follow-up (correlates of .49 and .34 , respectively). A quite different pattern was displayed for alcohol use. The frequency of alcohol use was barely determined by reported age of first use, and was not conditioned by the expired time between the waves. The intensity of alcohol consumption was significantly $(p<.05)$ associated with the reported age of initiation at wave one (.14), wave two (.08) and wave three (.17).

In conclusion, our findings reveal that the interpretation of associations of the 'age of onset' concept with later behavior yields substantial problems. Transversal relationships at wave three seemed to demonstrate the relevance of the initiation of cigarette use for the progression of use later on. When the associations of smoking (T3) and the apparently most correct indicator of the actual age of smoking onset were taken into account, however, no predictive value was found. Regarding alcohol use, the age of first use appeared to be of minor importance for use later on.

Table 10.2. Correllations of Age at First Use Reported at Three Waves with Intensity and Frequency of Alcohol and Cigarette Use at Wave Three

\begin{tabular}{lccl}
\hline Substance use & \multicolumn{3}{c}{ Age at first use } \\
\cline { 2 - 4 } in 1994 (wave 3) & 1989 & 1991 & 1994 \\
\hline Alcohol use & & $.08^{*}$ & $.09^{* *}$ \\
Frequency" & .07 & $.08^{* 4}$ & $.17^{* 4 *}$ \\
Intensity & $.14^{* * 4}$ & $.15^{* * *}$ & $.49^{* * * *}$ \\
Cigarette smoking & .05 & $.09^{* *}$ & $.34^{* * *}$ \\
Frequency & .05 & .06 & \\
Intensity & &
\end{tabular}

Note. $p<.05,{ }^{*} p<.01$, $p<.001 .{ }^{2}$ Positive coefficients signify that an earlier age of first alcohol use is associated with a higher frequency of use at wave three.

\section{Discussion}

In this paper the internal consistency of self-reports about the age of first alcohol and cigarette use by adolescents was analyzed. Information was gathered at three waves with intervals of two and three years respectively. Variations in the average 
age of onset at the waves, the magnitude of errors in estimations and the consequences of different estimates for associations with smoking and drinking at the last wave were examined. Findings and conclusions were based on a selection of subjects: only youngsters who indicated having smoked at the baseline measurement, and who responded to the question about age of initiation were included in analyses. In this section we will summarize the major findings, discuss the improvement of measurements, and address some possible explanations regarding errors in estimations.

Substantial differences were found when the average age of first use was calculated for each wave. Throughout the study, adolescents were more likely to report a higher age of initiation. Mean ages increased from 9.8 (T1), 12.02 (T2) to 13.53 (T3) for alcohol use. A similar pattern was displayed with respect to cigarette smoking. The reported age at first smoking rose from 10.52 (T1), 11.63 (T2) to 12.42 (T3). At the individual level, consistency rates were $13.6 \%$ for smoking, and $4.6 \%$ for alcohol use in a period of five years. Only a few subjects overestimated their years of substance use, but nearly $61.7 \%$ and $89 \%$ underestimated the timespan of tobacco and alcohol use respectively. Approximately 10\% (smoking) and $36 \%$ (drinking) of the sample demonstrated errors in estimations of 5 or more years.

Correlational analyses between the age of onset measures and actual behavior at wave three showed different effects. The highest associations were found when cross-sectional data of the third wave were analyzed. Only in one out of four cases (intensity of alcohol use) was age of onset reported at wave one correlated to actual behavior at wave three.

In conclusion, our findings clearly demonstrate not only the existence of substantial errors in estimations, but also stress the poor predictive value of the age of onset measures for recent smoking and drinking behavior of adolescents.

\section{Conceptualization and Assessment}

In this study, the age of initial use had only a minor predictive value for later smoking and drinking. Before omitting this variable as a determinant of substance use, additional assessments of early use which may lead to other outcomes, should be discussed.

Inaccurate responses might be due to changes in the definitions of what substance use is. Perhaps measurements of concepts should be age-related, and adjusted for changing interpretations and assumptions of youngsters. Moreover, the concept age of first use might be replaced by adopting the assessment of regular consumption. Young people may more easily be able to recall the time they started with, for instance, weekly consumption, and the starting point of weekly drinking seemed to be a better predictor of consumption patterns later on. Furthermore, in this study, subjects were asked to report their life-time tobacco use and age of onset of smoking, even if they smoked only a few puffs. For alcohol consumption, 
however, we first asked them if they ever drunk alcohol, even a sip and then we asked how old they were when they first tried alcohol beverages. Further research might focus on the consequences of different formats of questions about substance use on young people's responses.

The utilization of specific events in questionnaires may provide the opportunity to improve the quality of assessment, and obtain more accurate responses. A method to anchor events and drinking episodes is the Timeline Follow-Back technique (Sobell, Sobell, Leo \& Cancilla, 1988; Sobell \& Sobell, 1992). Among several categories like normal drinkers, heavy drinkers or liver disease patients, this method is one of the best to assess drinking history by self-reports. It would be interesting to examine whether this technique could be used for the recall of responses on smoking, drinking or illicit drug use among adolescent populations. For example, if adolescents are asked to remember personally relevant events such as birthdays, transitions in friendships, or specific dating-partners, and hence are questioned about their smoking and drinking habits in that period, more accurate responses could be obtained. Questions about situational (Where did you first $\mathrm{drink}$ an alcoholic beverage?) and social (With whom did you try your first cigarette?) aspects of first use might contribute to more precise responses as well. Another promising option is to employ discrete response categories. It is conceivable that people find it difficult to provide exact data on substance use patterns after a few years. Supplying answers in a fixed format might improve the capabilities of adolescents to classify their own behavior.

\section{Interpretations of Inconsistencies in Estimations}

One can only speculate on the causes of inconsistent estimates. Bailey et al. (1992, p. 645) suggest that high rates of estimation errors were partly due to '...greater precision requirements for the age measures'. The use of a stringent measurement of the age of onset in the current study might account for the small percentage of consistent responders. It is also possible that adolescents are more able to recall earlier frequency, consumption levels, or drunkenness than the exact time point of first use. Another interpretation is that adolescents change their definitions of smoking and drinking. For example, consuming only a few drinks at the age of 10 could be redefined consciously as non-use a few years later. Although in longitudinal research formulations of behavioral items are usually constant over waves, interpretations of these formulations by adolescents may change over time. A third interpretation refers to the episodic nature of experimentation with alcoholic beverages and cigarettes by youngsters. For instance, if a person drinks at a family party at the age of 10 , has a period of abstinence of three years, and then consumes alcohol with friends at the age of 13 , this person might remember only the last occasion and report it is as his or her first use. 
Although we assume that redefinitions of use are accountable for a substantial amount of errors in estimations, attention should also be focused upon a fourth interpretation; the possible positive functions of underestimation of years of substance use. For example, realizing how many years one has used a substance may lead to negative feelings, such as dissatisfaction, worries or distress. With respect to cigarette smoking, there is an extensive body of research, and widespread information about the negative health consequences of smoking, such as lung cancer and heart-related problems. Additionally, media campaigns are designed to emphasize the negative effects of alcohol abuse such as losing contact with friends, becoming aggressive and drunk driving. Adolescents who have feelings of uncertainty about the continuation of their habits, might have stronger needs to underestimate their years of substance use than adolescents who do not experience such feelings. Another explanation for the increase in reported age refers to environmental influences. Research indicates that norms and beliefs of peers and parents are of influence on individual behavior (Conrad, Flay \& Hill, 1992; Petraitis, Flay \& Miller, 1995). If adolescents' own norms and the norms they perceive their parents and friends to hold are strongly opposed to cigarette smoking and regular alcohol use, one would expect them to indicate a greater increase in age of first use than youngsters who experience less conflicting values or norms. Since only a small number of subjects provided consistent responses, we could not examine the reasons for consistent or inconsistent estimates. Research is, therefore, warranted to explore potential causes of errors in "age of onset" measures.

Findings of this study pointed out to a cautious approach when using the concept age of onset as a predictor of use later on, or when presuming that it is a reliable indicator of addiction to substances. Although the predictive value of this variable appeared to be limited, a search for explanations concerning errors in estimations of years of use is still required. Finally, it would be fruitful to replicate this study with a sample of adolescents, but focusing on the recall of different behavioral patterns.

For instance, people might remember their first kiss and sexual experiences very well. While a first sip of beer or wine could be an occurrence which did not stay in their memory for a long time. Besides behavioral aspects, further studies should concentrate on the effects of recalling earlier attitudes or opinions about specific topics. Although speculative, our findings could have implications for prevention efforts. Research on risk perceptions (e.g., Lopes, 1993) and the false consensus effect (Marks \& Miller, 1987) show that some youngsters do not consider themselves at risk for the negative effects of smoking or drinking on their personal well-being on the long run, or have incorrect beliefs about the prevalence of smoking in general or, more specifically, in their own peer group. This study indicate that most adolescent users also have an inadequate view on their own history of substance use. It would be worthwhile to pay more attention to these cognitions in health education programs. 


\section{Chapter 10}

\section{NOTES}

'Based on:"

Engels, R.C.M.E., Knibbe, R.A., \& Drop, M.J. (1997). Inconsistencies in adolescents' selfreports of initiation of alcohol and tobacco use. Addictive Behaviors, 22, 613-623. 


\title{
11 \\ GENERAL DISCUSSION
}

\author{
"In the middle of the front side of the rider is a pin \\ Turn it around twrelve times, and the gate will open." \\ (from Story of the City of Brass, \\ Tales from the Thousand and One Nights)
}

This research project was designed to examine predictors of changes in smoking and drinking behavior in adolescents. In this thesis, the focus is on the role of social environmental factors (such as parents, siblings, friends, peers and partners) in the development of substance use patterns. Furthermore, a relatively new path was explored by looking at the beneficial functions of drinking. It was assumed that, in order to gain insight into the reasons people have to drink, one should concentrate not only on the negative consequences but also on the positive sides. In addition, the relative value of individuals' opinions, intentions and skills regarding smoking in explaining specific transitions in smoking status (namely onset and quitting) was examined. The findings reported in the individual chapters are briefly summarized below (see also Figure 11.1). Some of the major issues in this thesis, namely social influence processes and substance use and functions of drinking and pub-going, are discussed in more detail. In particular, attention is paid to the shortcomings of our study and strategies to deal with them in future research. Furthermore, two important aspects in studying transitions in substance use, the impact of previous behavior and inconsistencies and errors in self-reports, are discussed. Some suggestions for applications to health education and policies are put forward. In addition, in order to deal with some of the limitations of this thesis (and of other studies) as well as to gain more theoretical insight into these topics, three plans of investigation for future research are presented. 


\section{SUMMARY OF FINDINGS}

The interpretation of similarities of substance use in peer groups and friendships is addressed in chapter 3. Data on changes in smoking behavior in adolescents and their immediate peer group, and changes in the construction of the peer group, over a three year period were used, to test the contribution of selection and influence processes on homogeneity in smoking. The central question is whether the strong association between smoking by an adolescent and his/her peers is due to the impact of peers on individual smoking, or to the selection by adolescents of friends on the basis of shared beliefs and/or behaviors. Our results showed that strong support was found for selective association, no support for deselection (i.e., breaking of friendships due to dissimilarities) and only weak support for influence processes. The notion of selection processes is used in the development of a structural model employed to test the relative effects of friends and parents on smoking and drinking behawior in the three waves of the study. In chapter 4, it was found that parents had a weak but significant impact on the smoking and drinking habits of their offspring. On the other hand, best friends' use had no effect on adolescent use over time. Interestingly, parental smoking and drinking behavior seem to affect their children's friends behavior over time. This was unexpected, because we assumed that possible parental effects on friends would be mediated by their children. On the other hand, it fits with our impression that the influence of parents does not end at their off-spring, but that they also affect their children's friendships choices.

One of the most prominent changes in adolescents' social life is related to dating, romantic affairs and development of steady relationships. Besides friends and family members, a partner is a potential socializing agent. However, only few researchers have studied the role of intimate partners in changes of adolescent substance use. In chapter 5 , the impact of partnership on changes in alcohol consumption between mid- and late adolescence was examined. Males with a partner reported the lowest increase in consumption compared to males without a current partner and males who had never had a partner. It should be noted that males with a partner reported drinking more glasses at home (compared to the other categories) and fewer glasses in public drinking places. Additionally, chapter 5 deals with drinking in mid-adolescence in association with the likelihood of becoming involved with a steady partner. It appeared that abstainers less often have a steady partner compared to drinkers (for both sexes).

Chapters 6 and 7 explicitly concentrate on the functions of drinking and pubgoing for late adolescents. Cross-sectional data of the third wave were used for analyses in both chapters. Previous studies have reported that drinking might have some beneficial consequences for youngsters; moderate drinkers are more apt to adopt more adult roles, are more advanced in their identity formation, have more and better contacts with their friends and report higher levels of psychological well- 
being than abstainers. Since these studies leave open the question of whether these beneficial effects should perhaps be ascribed to their presence in pubs, discos and parties instead of the characteristics of the beverage, we looked at the relationships between functions and pub-going. Non-visitors reported adopting less adult roles and being less socially integrated than visitors. Surprisingly, no differences were found between stress levels, self-esteem and pub-going. Chapter 7 aims to explore another function of drinking, namely drinking to alleviate negative feelings of stress and low self-worth from a contextual perspective. It was found that, in particular among males who exclusively drink at home, a higher level of alcohol use was related to enhanced stress and low self-esteem. For males who drink in public drinking places or in both situations, no significant assaciations in either direction between alcohol and stress were found. Additional analyses showed that male homedrinkers are less integrated in their peer group and have fewer experiences with steady partners than the other two categories. These effects were not found for girls.

There is an ongoing discussion about the efficacy of models to explain and predict adolescent smoking behavior. In chapter 8 , findings from cross-sectional and longitudinal analyses are compared. Smoking-specific cognitions, smoking by peers and family members, and socio-demographic variables were used as predictor variables. Although the cross-sectional analyses are rather optimistic, the finely grained prospective analyses concentrating on the transition from non-smoking to regular smoking indicate that only a few percent of the new smokers can be predicted by the model variables attitudes, self-efficacy and parental smoking. The study described in chapter 9 focuses on another transition in smoking behavior, from regular smoking to quitting. In this study, smokers were distinguished by their motivation to quit. Smoking attitudes, self-efficacy expectations and habitual factors (frequency and quantity of smoking, integration of smoking into daily life) affected young people's motivation to quit smoking. Those with positive smoking beliefs, low levels of self-efficacy, high frequency of use, high number of cigarettes smoked per week and high prevalence of smoking in various situations, are less motivated to quit. Social pressures or modelling (by family members, best friends, or the peer group) did not affect their motivation to quit.

Most empirical studies rely heavily on people's own reports about their individual preferences and behaviors. Without observing people's behavior it is rather difficult to check whether information on the history and actual smoking and drinking behaviors is valid and reliable. Chapter 10 describes a study on the reliability of the assessment of age at first tobacco and alcohol use. Correspondence methods were used to determine the accuracy of estimates by comparing estimates provided at three waves. In general, adolescents who indicated life-time prevalence at the first wave (and their age of onset) reported higher ages of onset at wave two and at three. Only a small proportion estimated a similar, or even lower, age of onset in a five year period. The unreliability of the age of onset assessment had its impact on the value of this concept as a predictor of later use. The presumed most 
reliable estimation of the actual age of first use (given at the first wave) is in most cases not rellated to smoking or drinking behavior at wave three.

Figure 11.1. A Summary of the Findings in this Thesis

- Wherher smoking and drinking behaviors can be predicted by earlier use

$\frac{\text { Chapter }}{2}$ depends on the measures and analytic strategies employed.

- Homogeneity of smoking behavior in peer groups shoulld to a large extent be ascribed to selection processes and to a lesser extent to influence processes.

- When selection processes are considered, parental smoking and drinking have more impact on changes in adolescent use than best friends" use.

- The impact of family members is more complicated than is often believed because parents not only have an effect on their off-spring but also on their choice of friends.

- Having a steady partner implies higher levels of use at home and lower levels of use in public drinking places (especially for malles).

- Pub-going is positively related to good integration in a peer group, involvement in an intimate relationship and other transitions marking the adoption of more adult roles.

- Late adolescents who visit pubs and discos show no better well-being than non-visitors.

- Alcohol use might be interpreted as a way to deal with negative emotions for males drinking exclusively at home but not for males who drink (also) in pubs and discos.

- The factors which strongly correlate with adolescent smoking (peer smoking, atritude, self-efficacy) at $\mathrm{t} 1$ are only weakly related to smoking onset three $(\mathrm{t} 2)$ or five ( $t 3)$ years later.

- Young people's motivation to quit smoking is associated with smoking attitudes, self-efficacy expectations and habits three years earlier.

- Large numbers of young smokers are absolutely not motivated to quit.

- The concept 'age of first use' should be utilized with caution because the relliability of assessment is insufficient, and correlations of age with actual smoking and drinking are inconsistent.

\section{SOCIAL INFLUENCE PROCEsses: SHORTCOMINGS IN OUR STUdies AND NEW DIRECTIONS}

A central question in this thesis is to what extent young people are affected by others in their immediate surroundings, such as parents, best friends, peer group, siblings and steady partners. To examine this issue, research should also focus on the extent to which similarities in smoking and drinking in social groups or pairs are due to selection and influence processes. Furthermore, we concentrate on the relations of social influences with substance-specific cognitions, such as attitudes, 
self-efficacy and intentions to smoke, and with socio-demographic factors. In this chapter, we aim to address the pitfalls in our research and elaborate on 'new' directions for the study of social influence processes.

\section{Shortcomings in Current Research on Influence and Selection Processes}

First, no information was acquired from subjects" friends in the present study. Besides the problem of projection which is discussed in chapter 4 , the absence of data from friends also precluded the opportunity to look into the structure of peer networks and to relate it to changes in substance use patterns. Second, to understand the dynamics in adolescents lives with many changes in social and personal life within sometimes just a few months, it is assumed that one or more years between measurements is perhaps too long. A study that monitors a group of youngsters for 1.5 years with intervals of no more than 3 months might provide more precise information about selection and influence processes. In such a design, it is also possible to examine the ordering of influence and selection processes in more detail. For instance, in the case of alcohol use, it is likely that on the one hand youngsters would seek others with similar opinions on drinking and consumption patterns; on the other hand, in a given drinking situation (a Saturday night in a disco) they are influenced by others in the number of glasses they consume that evening. Third, parents might affect the extent to which selection and influence processes occur. Parental rearing styles and communication in famillies affects how children act and behave in peer groups (Dekovic \& Janssens, 1992). For instance, if parents have strong anti-smoking norms and socialize their off-spring into nonsmoking, children might be more likely to stand up against peer pressure. On the other hand, if an adolescent grows up in a smoking family environment, $s /$ he might be less effective in handling pressure from peers. Asking adolescents about the role of parents directly might perhaps lead to incorrect and incomplete information. In order to gain accurate data one should include one (or both parents) in a network study.

The prevailing belief that smoking is primarily determined by peer influence conflicts with the findings of Ennett and Bauman (1993) who reported high taking up of smoking in social isolates. They have some explanations for this phenomenon. For instance, social isolation might cause smoking out of feelings of stress and boredom. Second, it is possible that smoking causes social isolation - a non-smoking peer environment might drive a smoking member out of the peer group. Our study (see chapter 3) and another study by Ennett and Bauman (1994) do not support deselection processes: youngsters do not lose group membership due to differences in smoking status. A third explanation might be that, because the authors employed their network approach only in schools, isolates actually have friends outside school who smoke. Although these explanations need further study, the high prevalence of 
smoking in social isolates demonstrates the relevance of unravelling social networks of youngsters in order to examine peer influences.

A problem in studying adolescent networks has to do with a culture of multiple peer groups: adolescents have friends at school, sport, in pubs and bars, scouting etcetera (see Mosbach \& Leventhal, 1988). A school approach might result in low numbers of 'exclusive" cliques, or in large numbers of isolates who have actually many friends outside their school. Inclusion of not just a sample of the target group, but the target group itself, will result in complete data on (changes in) social relationships. A study on a more-or-less 'close' community, such as among young inhabitants of one the Wadden Islands in the Netherlands might offer a solution.

Related to this latter point, cultural, ethnic or religious aspects are mostly neglected in the research on social influence processes. Peer networks, however, develop in a social and cultural context. For example, studies from the U.S. have shown that the majority of (early) adolescent friendships are formed at school (Ennett \& Bauman, 1994; Urberg et al., 1997). Whether this school is based on Catholic, Protestant or non-religious principles has its impact on the attitudes and life style of people entering these schools. In addition, young people spend a lot of time in their neighborhood. It is likely that in deprived areas with drug use and drug-related problems, young people have other ideas about, and experiences with, substance use than children who grow up in more privileged areas. Additionally, there could be regional differences in how youngsters act in their peer groups. Youngsters in rural areas (small villages) might have different situations in which they spend their free time, they might have fewer networks to become a member of and also might have different substance use patterns than those who live in urban areas. Although it is difficult to examine how, for instance, culture and religion are precisely related to selection and influence processes, one could start with exploration of the differences and similarities in operation of peer influences in different ethnic groups in the Netherlands.

\section{Windows of Vulnerability: Relative Influences of Parents, Peers and Partners}

Parents seem to have more effect on adolescent smoking and drinking than the peer group or best friends when selection processes with respect to similarities in peer groups are controlled for. It is important to say that it was not expected that one party on its own would affect changes in smoking and drinking: the relative influence of parents and peers was studied. In this respect, an interesting perspective on the changing influential agents in adolescence is suggested by Lau, Quadrel and Hartman (1990) in their work on young Americans" health beliefs. Their windows of vulnerability model assumes that parental influence on children's attitudes and behavior will persist throughout life unless the child is exposed to other influences during critical periods. According to the authors, in adolescence when youngsters 
try to become more independent from their parents, the time when people grown up and leave their home and the moment that they start their own family (get married, cohabiting, children) are three vulnerable periods. The model predicts that parental influences endure unless the adolescent is exposed, at that moment, to more relevant socializing agents (who differ in norms and opinions from parents). Whether the impact of friends or intimate partners in adolescence drives an individual away from his/her parents of course depends on the differences in attitudes on certain topics between significant others.

Adolescence is marked by an increasing need, capacity and opportunity for intimacy (e.g., Paul \& White, 1990). It is the time in which romantic relationships start which, besides good friends and the immediate peer group, have a function in the personal development of adolescents. In terms of influence processes, a new source of potential impact on substance use patterns becomes apparent in youngsters" lives. Our study showed that, in particular for males, involvement in a steady relationship affects drinking behavior. The increase in drinking levels in public drinking places is lower for young men with a steady partner than for those who are nor (or never have been) involved in a relationship. However, they seem to change their drinking pattern by consuming more alcohol at home. This phenomenon can be explained by a study of Silbereisen et al. (1992). The authors state that when youngsters have a partner, they spend more time in private settings and less time with other peers.

In our opinion, it is curious that not more emphasis is placed on the role of intimate partners in research and prevention. For the latter, an explanation might be that most programs focus on early adolescents (10-13 year olds) who have limited experiences with intimate relationships. However, if the goal of a program is to prepare adolescents against the pressure of peers to drink or smoke in the future, it might be worthwhile to think about the role of future partners more thoroughly. There are some issues to solve first. It is very likely that selection processes are accountable for many of the similarities in smoking and behaviors and attitudes within intimate relationships. In addition, it would be interesting to compare the impact of a partner, friends and family members in a study among late adolescents. Limitations in our data did not permit us to do further analyses on this topic.

\section{Mediating Factors Affecting Susceptibility to Peer Influences}

Although not much support was found for the hypothesized strong impact of peers in this thesis, this does not mean that peer factors should be neglected as a relevant source of influence. In our opinion, future attention should be focused on the conditions under which peer influences are apparent. The ways people react to pressure by peers to smoke or drink depend not only on the amount (or type) of pressure exhibited. For example, personal and group characteristics might also affect young people"s susceptibility to peer influences. 
Bruun (1959) observed drinking in small groups and examined the importance of group cohesion on drinking patterns among adults. Cohesiveness tends to homogenize opinions and attitudes of group members. Among the most important beliefs that emerge from extended interaction in groups are the rules of conduct that define appropriate or expected behaviors of group members (Selnow \& Crano, 1986). Consistent with this reasoning, Johnson (1980) postulated that belonging to a group while receiving acceptance and social support from group members contributes to sensitivity to the needs of others, and to keeping the group together. 'Thus, it is postulated that high group cohesion would result in relative homogenous drinking patterns. In addition, variations in dominance, prestige and control among group members resulting in status relations reflect an important aspect of people's functioning (Doreian, 1986). Studies on social standing of adolescents in classes (e.g., Wright et al., 1986) revealed that individuals in a group setting fall into different categories, resulting, for instance, in a classification of popular, isolates, outcasts and average members. Higher status within a group is associated with a stronger control on communication patterns in the group, higher attractiveness and acceptability among other members, and stronger impact on people's choices and performances (Forsyth, 1990).

Personality factors also affect how people react to peer pressures. Perhaps young people who suffer from low self-esteem have a stronger desire to belong to a certain group and are more prone to conform to influences of friends. Simons, Conger and Whitbeck (1988) extended these assumptions by adding social skills to the examination of the relations between distress, low self-esteem and substance use. The way young people handle social forces (for instance, by negotiation or avoidance) is expected to have an effect on the extent to which they conform to peer influences. Besides the uncertainty of adolescents on how they function (or think they should) in peer groups, they differ also in their needs to belong to a certain group. The motivation to affiliate, defined as the tendency to enjoy being with friends, to make efforts to win friendships and to maintain associations with others (Wong \& Csikszentmihalyi, 1991) affects people's performances in social situations. It would be interesting to look for conditions under which adolescents are likely to be vulnerable to peer influences. Information might lead to the possibility of tracing categories which are at risk and to offer effective tools for skills training for these youngsters. In the section 'New Directions' a project proposal on this topic is discussed.

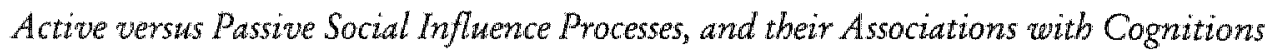

The focus in this thesis was on modelling. Only in the paper on smoking cessation (chapter 8) was an attempt made to compare the impact of active and passive social pressures. There are several reasons for the limited attention given to unravelling different types of influences. First, a question on explicit pressures to smoke or 
drink resulted in a very low variation in the scores, especially at the first wave. Because only a few adolescents, and thus also their peers, smoked at the age of 11 and 12, explicit pressures and offers were relatively rare. Furthermore, it seemed that at these ages parents did not offer cigarettes or alcoholic beverages to their children, and if they did, it was only done when their off-spring already smoked or used alcohol. Another reason for the low variation in the measurement of peer pressure might have to do with the wording of the questions. Simply asking youngsters whether they experienced pressure from significant others might not be very effective in getting valid information about actual social pressures (Friedman, Lichtenstein \& Biglan, 1985). Another more practical reason is that other kinds of social influences, such as norms on smoking and quitting, and the amount of social support during a quitting attempt, which are expected to be relevant factors (Cohen \& Lichtenstein, 1990; Petraitis et al., 1995) were not included in the (first two) questionnaires. Fourth, for the analyses in which we also concentrated on selection processes, incorporation of other forms of social influences available in our data-set (pressures and offers) would perhaps make an analytical model too complicated.

Flay et al. (1994) tested a longitudinal model in which effects of peer and parental smoking on changes in adolescent smoking were mediated by attitudes and self-efficacy. They found an independent effect of peer factors, but not of parents, on smoking status. This result coincides with the study of De Vries et al. (1995) in which also strong effects of modelling variables were found even when (other) cognitions ${ }^{1}$ had entered the equation. It should be said, however, that neither study included the possibility of selection effects. Although we concentrated on the associations between attitudes and self-efficacy, and social influences, no explicit hierarchical model was tested. It should be interesting to explore the merits of hierarchical models, as well as possible differential effects of different types of social influences, when a control for selection effects is included.

\section{FUNCTIONS OF ALCOHOL CONSUMPTION AND PUB-GOING}

The papers on the beneficial functions of drinking are to some extent aimed as a reaction to the overwhelming research on negative consequences of drinking by young people. In essence, these studies do not give us many clues about the reasons people have for going out and consuming alcohol. In our research several social and personal aspects which can be ascribed to drinking, were distinguished. Furthermore, to understand purposes of drinking, the situations in which youngsters commonly drink become highly relevant. In the following section, we stress the limitations of our study and highlight directions for future research. 


\section{Explarative Aspects of our Studies}

Before elaborate on the findings, it should be clear that the papers on functions of pub-going and drinking (chapters 5 to 7 ) are the most explorative ones in this thesis, in particular because of the cross-sectional analyses. It is, therefore, not possiblle to draw conclusions with respect to the causal nature of different processes. Is the increase of youngsters ${ }^{i}$ social networks a result of visits to public drinking places or are those who are well integrated in their peer group more likely to meet each other in social settings such as pubs and disco's? This study cannot address the long term effects of a limited exposition to social situations, but displays the functions of pub-going at a specific time point. Future research must explore aspects of etiology in more detail. Second, the design of this study was not specifically prepared for all research questions that emerged during the analyses. Interest in school success, for example, is not a detailed and precise measure. Data on actual school performance and more in-depth information on value orientations and future ambitions would provide more insight on late adolescents" values and positions in life. In addition, more detailed data on the quality of friendships, on the aspects of friendships which are related to drinking and on relationships with parents and siblings would present a more complete picture. Reference persons, such as parents, friends and teachers can provide detailed information on the (social) functioning of the respondent which contributes to the validity of the data.

\section{Insights into Functions of Drinking}

Our research on functions of pub-going, and the relationships between drinking and partnership suggest that entrance into alcohol-related settings has its benefits. Late adolescent visitors of public drinking places report having more friends, better relationships with their friends, more often a steady partner and are more inclined to adopt adult roles than non-visitors. In addition, youngsters who drink in midadolescence are more likely to have experiences with steady relationships in lateadolescence than those who do not drink. Furthermore, it was expected that visitors would have less stress and higher self-esteem. However, our findings showed that there were no differences between visitors and non-visitors based on these factors.

The fact that we found significant differences between visitors and nonvisitors in the quality of friendships, but not in stress and self-esteem, might suggest that general scales do not provide sufficient specific information. For instance, detailed questions about young people's self-worth in the company of friends, schoolmates, eventual partner or parents could be more effective in showing how pub-going is related to self-esteem. Other explanations are related to the age categories included in the study. Perhaps in young adulthood, people who for a long period of time do not visit places with social functions, might have psychological problems. On the other hand, selection processes might result in non-visitors 
seeking company with people holding similar opinions and behaviors, and thus behaving quite satisfactorily in these peer groups.

The paper on beneficial functions of drinking tries to structure these functions by distinguishing dimensions, namely, other transitions marking the adoption of adult roles, social integration and well-being. Despite the correlations between dimensions, it is assumed that it is fruitful to work out these dimensions more thoroughly. It appeared that the items in the three dimensions did not correlate well highly enough to form one coherent scale. A reason for this instability lies in the diversity of the contents of the items. Further research is required to develop valid and reliable scales.

A second, related, problem is that it remains unknown whether the function of drinking is similar to the function of pub-going. Although our research can not be conclusive about this issue, the study on relationships between psychological well-being and context of drinking (chapter 7 ) shows that most likely the context of alcohol use is essential. Functions of drinking vary by context; for those who drink at home, social purposes (enhancing contacts with friends and other peers) are less relevant than for those who drink primarily in public drinking places. Furthermore, the strong association between drinking and pub-going (see also Knibbe et al., 1991) makes it difficult to unravel whether certain functions are more related to the setting and others more related to drinking. For instance, if someone drinks in settings without the presence of the opposite sex, drinking will hardly increase the likelihood of development of romantic relationships. On the other hand, drinking in public drinking places might give people the feeling of becoming more free and less inhibited to approach others.

Chapter 7 aims to explore under which conditions people use alcohol as a way to cope with feelings of stress and low-esteem. Findings displayed that, in general, late adolescents who drink heavily or moderately do not report more stress and lower self-esteem than light drinkers. However, when subjects are distinguished by categories of context, it appeared that only for those who drink exclusively at home are drinking levels related to stress and low self-esteem. The results need further elaboration and explanation. Little is known about the underlying mechanisms (roles of coping styles, social support, or parental norms and drinking patterns) and the stability of the relationship between well-being and drinking in the category of male home drinkers (for instance, how is their social position and functioning a few years later?). Nevertheless, the findings underscore the relevance of looking for categories of youngsters for which drinking has few social benefits, but is related to poor psychological well-being.

\section{Expectancies and Outcomes}

Although there is an extensive amount of research about the beliefs concerning social consequences of alcohol consumption, only a few studies focused on the 
actual outcomes of these beliefs, and their subsequent effects on beliefs and drinking. If one's alcohol consumption is affected by the expected outcome, for example, feeling more free to engage in romantic activities, and one remains empty. handed after a couple of nights in the disco, attitudes and behavioral patterns might change later on. Leigh and Aas (1995) stated that before stable drinking patterns are established, experiences with the effects of alcohol have substantial impact on expectancies and behavior later on (see also Stacy et al., 1994). Thus, the reciprocal processes between expectancies, behavior and outcomes should be studied to get insight into the continuation and stabilization of drinking among adolescents.

\section{Drinking as Part of a Life Style}

Smoking and drinking might be indicative of a certain life style. These behaviors do not stand alone but are imbedded in value orientations and behaviors which are in most cases related to social membership. Music choice, type of clothes, communication styles in a group (with own slang), specific leisure time use, food preferences and also the use of certain substances are resulting in characteristics of a group. This means that, for instance, the longitudinal relationship between drinking and involvement in steady relationships does not necessarily imply a causal relationship. It is more likely that drinking and, for instance, early interests in romantic relationships in mid-adolescence are aspects of a life style of pre-mature adolescents.

\section{Drinking as a Consideration of Positive and Negative Aspects}

Only limited attention is paid to the negative sides of drinking (except chapter 7). This does not imply that we have a favourable standpoint towards use. In our opinion, alcohol misuse and its adverse consequences should be prevented. Still, research on positive aspects of adolescent drinking is scarce, and is essential for the understanding of youngsters' reasons for taking up drinking and the continuation of drinking patterns. Investigation of the effects of consequences of alcohol on the subsequent attitudes and behaviors requires information about the contemplation process of both negative and positive consequences. If an individual reports some positive consequences of drinking but, at the same time, the negative consequences out-weigh the positive consequences, this person might be more likely to change his/her drinking patterns (see Mäkelä and Mustonen, 1996). In addition, one might conclude that abstaining and continued absence in alcohol-related settings are related to a deprived social and personal position. However, as we did not include the beneficial sides of abstinence, and the negative sides of drinking, such a conclusion is premature. 


\section{IMPACT OF HABITS}

In chapter 2, the question of whether people move through certain stages of smoking and drinking onset was explored. This paper, however, had limited value in examination of the merits of a stage approach (mostly due to the large intervals between the waves which do not take into account the dynamic nature of the process of onset). Only with a study which monitors smoking and drinking behavior of a group of adolescents can a better understanding be expected. Previous studies have shown that it is quite reasonable to assume that different processes are involved in stages of smoking and drinking. Perhaps the best example is the strong impact of habitual factors on young people's motivation to quit smoking. Of course, in the transition from non-smoking to experimental smoking this factor is of no value, and other factors are influential.

We assume that it is important to study how young people go through subsequent (motivational) stages of onset and motivational stages of quitting. Werch and Anzalone (1995) proposed a model which combines stages of onset and cessation. In particular for adolescents, who mostly have been smoking for a short period of time, differences in motivational stages of cessation might also reflect different positions in the stages of onset. Since Werch and Anzalone did not test their model empirically, further research is warranted. A second issue is the restriction to behavioral aspects of smoking and drinking in stages of onset. According to Hirschman et al. (1984) one of the first stages of onset is a shift toward positive smoking attitudes and the perception of fewer barriers to start smoking. Our paper on predictability of smoking onset (chapter 9) showed that smoking attitudes in early adolescence are of predictive value for onset later in adolescence. To what extent attitudes, and perhaps other factors, are preceding actual onset or other transitions should be subject for new research.

Despite the current lack of knowledge on stage approaches, and the stability of behavior over time, the interpretation of findings will be enhanced if analyses are focused on specific transitions. An often applied analytic strategy, namely the prediction of smoking or drinking at 'T2 corrected for behavior at T1, offers limited information; in most cases no insight is gained into which other variables at 'T'1 are related to certain transitions in substance use.

Neglecting the role of previous behavior in predicting actual adolescent substance use might lead to incorrect conclusions. Predictor variables which correlated with behavior at $\mathrm{T} 1$, were more strongly related to use at $\mathrm{T} 2$ in a multivariate analysis if there was no correction for use at T'1. In orher words, the impact of other predictor variables could be overestimated. In addition, one might question whether changes in smoking and drinking are determined by a deliberate decision process (see also chapter 1). Habitual behaviors, such as regular smoking, have become so routine through repetition that they no longer require conscious decisions to act (Triandis, 1980). In sum, many social psychological and sociological 
models, such as expectancy value models and rational choice theories do not include the effects of previous acts properly. Especially for behaviors which are of an addictive nature, it is essential to include habitual aspects in order to comprehend processes of change.

\section{INCONSISTENCIES IN SELF-REPORTS}

Our paper on inconsistencies in self-reports of age at which smoking and drinking were initiated demonstrates possible problems with the validity and reliability of self-reports in cross-sectional surveys. When people are asked to give estimations of their age at first use of cigarettes or alcohol, in particular when the moment of questioning is a long time after the actual age of first use, substantial errors are apparent in their estimates (see chapter 10). However, it should be mentioned that, in our study, we used a single question to measure this construct. It is possible that a more extensive assessment would lead to more accurate responses. An adaption of the 'Time Line Following Back method (e.g., Sobell et al., 1992) might be useful. Inclusion of 'life events' (e.g., school-related issues, changes in specific friendships or romantic relationships, changes in relationship with parents, changes in activities in their leisure time) and questions about specific situations would perhaps result in more accurate data.

Our data could also suffer from other inconsistencies. People might, for instance, provide incorrect information about their drinking levels or smoking status. This may be on purpose, because they are afraid that others (peers, teachers, parents) might find out about their life style. In this study, we tried to decrease this effect by guaranteeing anonymity. Only the principal researcher was able to match numbers with names. In addition, the questionnaires were filled out without the presence of others. This was, however, a problem in the telephone interviews. Although respondents were asked to take care to be alone when the interview was conducted, we do not know whether other family members were present. Furthermore, characteristics of the interviewers themselves (in most cases women in their forties) could be accountable for some over- or under-reporting. Possible under- or over-reporting in the telephone interviews could not be checked because the sample interviewed by phone was already a distinctive category.

Youngsters might also unintentionally err in their responses. The measurement of the intensity of smoking was done by asking respondents to recall their cigarette use per week with a distinction between hand rolled and manufactured cigarettes. Several mistakes can be made. First, when a youngster primarily smokes at the weekends, s/he might forget the cigarettes on the weekdays. Second, we did not ask whether they inhaled the smoke or whether they always smoked the whole cigarette or only parts of it. Third, the fact that peeks were found on certain numbers $(5,10,20)$ might lead to the conclusion that people estimate their mean weekly consumption. Fourth, for people who only infrequently 
use cigarettes it might be more difficult to recall the precise number of cigarettes (or occasions). A similar argument applies to heavy smokers who smoke 15 or 20 cigarettes a day. Fifth, to get a picture of the quantity of use in a longer time period (for example, a month or a year) it is questionable whether extrapolation of the consumption in one week provides accurate information. Of course, most of these potential errors also apply to the measurement of alcohol use.

In spite of these facts, it should be clear that the main aim of this thesis was not the description of levels of adolescent smoking and drinking in the Netherlands. The design of the study also did not permit us to draw such conclusions. Still, if different types of users make different errors, for instance, if low consumers tend to overestimate their use and heavy consumers tend to underestimate their use, or vice versa, analyses on processes affecting changes in smoking and drinking should be handled with caution. We cannot say to what degree our data and findings are affected by errors and inconsistencies in self-reports.

\section{PREVENTION}

Regarding the credibility of social sciences, Schaalma (1995) stated that it will improve if researchers, interested in development of theory, do not end their articles with recommendations for the development of prevention programs. In other words, let the cobbler stick to his last. To a certain extent, we agree. Nonetheless, we would like to discuss the practical implications of some of the findings in our study. Our limited experience in applying theoretical concepts to health education and policy purposes leads to ad hoc ideas, suggestions and questions derived from our studies rather than well worked out guidelines for the development of effective programs.

First, it is important to discuss the eventual outcome of prevention efforts. These endpoints differ markedly by substance in the Netherlands. For smoking, the aim of prevention is to prevent as many people as possible from smoking. This implies that for non-smokers, the efforts are concentrating on continuing non-use while for regular smokers, the efforts are concentrating on quitting. Harm reduction, and not abstinence, is the target in the case of alcohol consumption. Thus, interventions are focused on bringing down the negative consequences of drinking among adolescents. Of course one of the reasons for the differences in policy, and norms in society, between substances, has to do with the variation in consequences for health. It is well known that smoking contributes to the risk of developing diseases like cancer and cardiovascular diseases, while moderate drinking is assumed to have no negative health consequences. On the contrary, it is often believed that moderate drinking has some protective effects for coronary heart diseases although the evidence for this assumption has been seriously criticized (see Lemmens \& Drop, 1997). 


\section{Predictability of Smoking}

The model variables we used to predict smoking onset correlated highly with smoking status cross-sectionally, but were hardly able to predict smoking onset over periods of three and five years. This implies that intervention in the last years of primary education and the first years of secondary education, which are based on factors employed in our study, are of limited value in affecting smoking a few years later. Exposing young people to a skills training or to an educational program on one or even a few occasions, is probably not enough to prevent them from smoking. Throughout adolescence, young people experience many new environmental forces and pressures which are long ago from the program in which they were once involved.

Nevertheless, this does not mean that health education does not help in the prevention of smoking. We assume that the effectiveness of programs improves if some considerations are taken into account. For instance, the high correlations on cross-sectional level plead for repetition. The incorporation of smoking prevention in the curricula throughout secondary education ( 4 to 6 times a year) might be helpful. In a quasi-experimental study, one could test whether schools with integrated health education in their program have lower incidence of smoking than schools with only a program at the beginning of students" secondary education.

Our study indicates that initiation of smoking might take place in a short period of time (at a critical moment) which is different for each individual. This idea of 'critical moments' implies that when a prevention program is performed, it will not suit all students. For some it is too late (they already smoke), while for others it offers rather new and unrealistic information. To give an example, when children aged $9-10$ are asked about smoking the majority are truly against it and strongly believe that they will never start smoking (see Goddard, 1990). Many of them, however, start to smoke after all. We assume that a lack of experience with the tempting situations or critical moments accounts for much of their earlier confidence.

\section{Social Interaction}

"Young people belong to groups (or want to) and such groups, within limits, are of their own choosing. In an important sense, young people choose the influences they experience. If intervention programmes are to be educational in more than a name, their role may be to help young people achieve greater self-awareness about their choice of friends, and about the mutual influence processes which such friendships imply" (Eiser et al., 1991).

Many programs have been designed to improve resistance skills and make youth aware of peer influences. The limited support for peer influences, and the strong impact of selection processes, in our project and in other studies (see review by Bauman \& Ennett, 1996) may lead to a reconsideration of the content of these 
programs. Selection processes imply that people actively choose persons to associate with, and that this company has similar opinions and behaviors. It is, however, rather difficult to judge how selection processes can be incorporated in programs. The suggestion of Eiser et al. (1991) that programs should make youngsters aware of their own thoughts regarding current and future friendships choices might be easier said than done.

We assume that there are individual differences in youngsters' vulnerability to peer influences. If further studies will demonstrate that, for instance, persons with low self-esteem and a high need for social affiliation are more prone to adopt certain smoking or drinking habits from their social environment, it should be interesting to explore whether programs focusing on specific categories of youngsters will be more successful.

In late adolescence particularly, intimate partner's norms and behaviors will perhaps have more effect than those of parents and friends. So far, limited attention has been drawn to the impact of dating and romantic relationships. Our study, which illustrates the reducing effects of a partner on boys' drinking levels, is a start. It would be interesting to examine the relative effects of a partner, as well as the role of selection processes in this respect, on adolescents' behavior.

That parents do play a small but significant role in the development of smoking and drinking habits, stresses the relevance of including family factors in health promotion programs. For example, community based programs directed at both parents and their children might result in lower uptake among adolescents, higher quitting rates among adults and a reduction of role models. We assume that organizational and financial aspects are accountable for the relative lack of attention given to parents in current Dutch programs. To give an example for its necessity: the Dutch Foundation for Smoking and Health has started a prevention program oriented on forming a non-smoking contract with 'all' students in a school class. In this way, students can keep each other on their agreements and negative peer pressure will be reduced. On the other hand, for youngsters with smoking parents or siblings, it might be more difficult to refrain from smoking because they probably do not get much social support at home for their efforts. In addition, there is also a lot of misunderstanding about opinions and norms held by parents about their offsprings' peers. Leventhal, Keeshan, Baker and Wetter (1991) suggested enhancing the dialogue between adolescents, schoolmates, friends and parent which might facilitate a better understanding of the decisions, influences and feelings of youngsters.

\section{Adolescent Smoking Cessation}

The research on smoking cessation came up with important issues. First, many young smokers have absolutely no intention of quitting. Since a high motivation to quit is an essential requisite for successful quitting, one should think about how 
adolescents can be motivated to quit. Furthermore, our study and others have shown that tobacco dependence makes it more difficult not only for adults, but also for adolescents who have only smoked for a short period of time, to quit. Perhaps programs should include elements which deal with the addiction (Burton, 1994; Prokhorov, Pallonen, Fava, Ding \& Niaura, 1996). How people think about the pros and cons of smoking, as well as their confidence in the success of an eventual attempt to quit, affects their motivation to quit. Since these factors are also found in adult samples (e.g., Dijkstra et al., 1996; Willemsen, 1997), it would be interesting to look at how adult smoking cessation programs can be adapted for adolescent smokers.

\section{Beneficial Effects of Drinking}

"... that it be not given to Youths, as from 14 years of age unto 25 , for wine is unto the most repugnant; because it doth above measure heat their hastie, hot, and agitating nature, and excimulate them (like mad men) unto enormious and outragious actions." (rules to live a long and healthy life by dr. Th. Venner, 1650)

For the majority of late adolescents, drinking constitutes a rite of passage. Youngsters come together with their friends or/and partner in specific settings (pubs, discos, parties) and at specific moments of the week (mostly Friday and Saturday evenings). In this situation they can forget the daily hassles by having a good time in the company of others. Most parents do not object to this way of leisure time spending by their offspring. On the contrary, many of them do not want to see their off-spring obedient and 'lonely' at home all the time. Of course these norms are partly based on the assumption that adolescent drinking habits are temporary. When youngsters grow older and acquire adult roles, such as living on their own, going to work and having their own family, the drinking habits of most people rapidly change, generally resulting in lower levels of use. In our opinion, one should concentrate prewention, as well as research, on the dark areas between situations in which social drinking takes place and situations in which drinking leads to several negative consequences (aggression, drunk driving) or situations in which people drink to cope with negative feelings and emotions.

Minimum Age of Drinking. In our opinion, steps towards further legislation on adolescent drinking, for example, raising the minimum age for accessing public drinking places, could have some negative consequences (see chapter 6). Young people will still seek opportunities to meet each other in places (such as in the street, shopping centers, at a friend's home without the company of adults) in which alcohol consumption might be less controllable than in public drinking places. Although it might sound paradoxical, an advantage of the drinking habits of adolescents remains in the fact that they are concentrated in specific public settings. Furthermore, the majority of late adolescents visit pubs and discos in the Netherlands. This large proportion implies that pub-going is not only socially 
acceptable behavior but also normative. Therefore, health education which aims to discourage people visiting these settings can be expected to have limited effects.

Changing Situational Characteristics. One strategy to reduce levels of alcohol use could exist in changing aspects of the drinking situation while maintaining the purpose of the setting. Observational studies suggest that variations in alcohol consumption partly depend on the tempo of the music (Bach \& Schaefer, 1979), loudness of music (Van de Goor, 1990), the seating arrangements (Cutler \& Storm, 1975) and the sex-ratio of the visitors (Knibbe, Van de Goor \& Drop, 1993). At meeting of experts on alcohol prevention, Knibbe (1998) underscored the relevance of attracting women to public drinking places because the presence of women in drinking groups results in lower drinking levels among men. Perhaps it might sound somewhat controversial, but it might slow down the fast drinking rates in large groups of young men. In addition, creating opportunities to play games or to dance might affect overall consumption levels. Policies which, in cooperation with the local authorities and owners of bars and discos, try to focus on contextual characteristics of the drinking setting have potential value. There is, unfortunately, only little scientific research done on situational characteristics and alcohol consumption.

Economic Actions. Youngsters spend a lot of money when they go out (mostly on drinks and fast food). Buisman and Esseveld (1988) postulated that increasing the costs of alcoholic beverages as well as reducing the cost of non-alcoholic beverages might be a tool for the reduction of high levels of drinking. Furthermore, pubs and discos often use 'free' offers of drinks and 'happy hours' to attract people to their place. If this were not allowed, it might contribute significantly to the prevention of alcohol misuse.

Reasons for Drinking. People might still have different reasons for visiting public drinking places. Perhaps the majority of young people visit pubs and discos, and subsequently drink alcohol, for social reasons (e.g., Christiansen \& Goldman, 1983). They might be in control of their drinking habits and do not suffer from any acute or chronic negative effects. Those who judge drinking places as primarily related to alcohol, and who also have robust and positive beliefs about the effects of alcohol, might be the ones with high consumption patterns and problems later on. Wiers (1998) differentiated low and high dose expectancies in adolescents. He stressed that interventions should aim in particular at countering strong positive expectations about the effects of high doses of alcohol in secondary school students rather than concentrating on alcohol use in general. In addition to this issue, our study 'searching' for drinkers who use alcohol as a coping style to alleviate feelings of stress and low self-esteem, suggests that for male 'home drinkers", drinking levels are related to distress. Although further research is required on this category of drinkers, it certainly underlines the necessity for distinguishing categories of youngsters on drinking levels and expectations about the consequences of alcohol. 
Avoidance of Negative Consequences. In terms of social consequences, a point of concern is the number of youngsters who become involved in fights and violence after the use of alcohol in pubs and discos. Police surveillance and efficient transportation (busses, taxis) to and from the scene might help to prevent criminal acts (Leegwater, 1996). Furthermore, many of the problems at the night occur when people are leaving pubs and discos to go on to fast food restaurants before they go home. Perhaps public drinking places should contain small restaurants to keep visitors in one place. If one aims to use educational tools to prevent negative

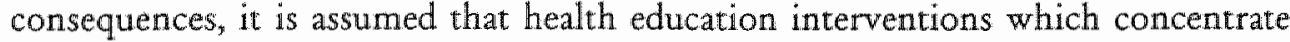
on risk-prone categories of youngsters are more effective in the prevention of certain side effects than school-based programs which concentrate on youngsters in general.

\section{DIRECTIONS FOR FUTURE RESEARCH}

\section{Why Not a General Model}

In their review of 14 theories on initiation of smoking, drinking and drug use, Petraitis et al. (1995) proposed a general theory which combined most of the factors in these theories. "This general theory postulates that adolescent substance use is determined by proximal, distal and ultimate influences. Proximal influences are the substance related cognitions from the theory of planned behavior (attitudes, selfefficacy, norms and intentions; Ajzen, 1991). Examples of distal variables are selfesteem, stress and academic skills, and attitudes and behavior of role models. Ultimate factors consist of local crime and employment rates or opportunities for rewards from family members, general life style elements or effects of prevention programs. A fundamental proposition of this model is that the effects of ultimate factors on behavior run through distal factors and subsequently through proximal factors.

Although it is tempting to postulate a general modell which includes almost all possible factors, it also has its disadvantages. First, such a comprehensive model is hardly open to empirical testing. Only relations between a distinctive set of variables and behavior can be properly examined. Second, it is not a dynamic model; it says little about (a) how predictor variables are interrelated and (b) how changes in behavior affect predictor variables. To give an example; starting to drink affects how people experience the positive and negative consequences of alcohol use and has its effect on attitudes towards alcohol. Furthermore, the positive social consequences might affect the way youngsters function in their peer group and their self-esteem. In addition to this latter point, some researchers have proposed that predictors might vary by the type of transition in behavior (e.g., Chassin et al., 1986; Flay et al., 1994). For example, a relevant factor affecting the transition from occasional smoking to regular smoking includes habitual factors (frequency and 
intensity of smoking, situations of use) which do not count for the transition from never-smoking to experimental smoking. Third, the role of selection processes in measurement of the effects of norms and behavior of others is not stipulated. Fourth, presumably one of the most important observations is that processes explaining changes in use might vary strongly in certain subgroups. Thus, educational level might not only affect people's attitudes and self-efficacy expectations towards smoking; in different educational categories different processes might be operating.

In our opinion, such a general model has its benefits on the descriptive level particularly. It summarizes the state of the art on predictors of smoking. However, to gain insight into processes of onset, it would be better to formulate specific and limited modells and hypotheses.

\section{Three Plans of Investigation}

Three ideas derived from the studies in this thesis will be worked out in more detail. ${ }^{2}$ Although we aimed to attack prevailing shortcomings in these studies and other studies (as are mentioned earlier in this chapter), it should be pointed out that these proposals can deal with only a few aspects. Additionally, the 'projects' are relatively independent of each other; the common feature is that they deal with adolescent substance use.

First, a proposal for a quasi-experimental study of the conditions under which youngsters are susceptible to peer influences is discussed. Central is the question of which combination(s) of personal and group characteristics leads to enhanced drinking and to smoking onset. Second, a longitudinal study which follows late adolescents into young adulthood aims to examine the consequences of adolescent abstinence and alcohol use in young adulthood. Third, a prospective 'monitoring' study should examine the dynamic processes of adolescent smoking cessation in more detail. The focus is on assessing the plausibility of a stage approach in adolescent samples and on possible predictors of stage-transitions.

\section{Effects of Personal and Group Characteristics on Susceptibility to Peer Influences}

Longitudinal studies on homogeneity of substance use in peer groups have shown that selection processes account for many of the similarities in use (e.g., Bauman $\&$ Ennett, 1996; Eiser et al., 1991; Ennett \& Bauman, 1994; Kandel, 1978; chapter 3 and 4). The value of peer influence as the most important predictor of adolescent substance use seems to need reconsideration in theoretical models and prevention practices. However, the fact that social network studies find only limited evidence for peer influences, raises the question whether peer factors should be dismissed as relevant in initiation and maintenance of smoking and drinking. Nonetheless, existing shortcomings in these studies should be corrected before more definite 
conclusions can be drawn. The present study deals with three of these shortcomings namely (a) little is known about individual differences in susceptibility for peer pressures, (b) research with questionnaires might not be the appropriate method to examine social influence processes and (c) little is known about people's other (emotional and rational) responses to peer pressure besides, of course, whether they take the cigarette or drink, or not.

Factors which might affect youngsters' susceptibility to peer influences are scarcely a subject for research. Personal characteristics (e.g., sociability, self-esteem, stress, anxiety) determine how people behave in groups and how they adjust to others (e.g., Ennett \& Bauman, 1994; Petraitis et al., 1995). Additionally, group factors such as cohesion, social status and norms, have an impact on people's intentions to change their substance use patterns (e.g., Turner, 1987). A systematic approach is required to test combinations of personal and group factors.

Getting reliable responses about social influence processes in adolescent lives by means of questionnaires is rather difficult. The research setting is far away from the setting in which peer influences operate (parties, pubs, discos, youth centers, school). The ideal design is an experimental study in which behavior in real settings is observed. However, the independent variables in such a study can not be manipulated convincingly. An alternative is a quasi-experimental design wherein respondents are confronted with vignettes (story narratives) which vary according to group factors.

To know how social influence processes operate, information is required on factors affecting people's responses to peer pressure. Affective responses, communicative reactions (coping styles) and distal reactions (avoiding the situation, seeking other company) might help to understand these processes.

"This project will have two phases. The first phase includes semi-structured interviews $(n=20)$ and focus group interviews $(n=32)$ writh non-users and users to gain more insight into the characteristics of the situation in which first use takes place. Special attention will be paid to participation of different categories of adolescents in this qualitative study. The second phase consists of two series of 3 experiments which concentrate on the conditions which inhibit or exhibit alcohol consumption and cigarette smoking. Each experiment has a $2 \times 2 \times 2$ factorial design. The experimentally manipulated variables are social status, cohesiveness or social pressure; sociability and self-esteem are the within-subject factors. Three combinations of these five factors will be considered in the three experiments. The designs of the experiments are identical for smoking and drinking. In total, 900 boys and girls in the 12-16-yrear age category will participate in the experiments.

Each experiment consists of a pre-test, vignette and post-test. For the pre-test, subjects will be asked to fill out a questionnaire containing scales concerned with substance use, personal and group characteristics and possible confounders (such as parental use, quality of social relations, outcome expectancies). The score on the within-subject variable will be computed. On the basis of allocation to one 
condition (for instance, low and high self-esteem) subjects will be randomly assigned to the manipulated factor. In a second session, 4 weeks later, subjects will be asked the vignette and fill out the post-test questionnaire. The vignette describes a recognizable situation in which a person is encouraged by friends to smoke a cigarette or to continue drinking. In the post-test, subjects are asked how they would act in this situation. Manipulation checks and process variables are also included.

\section{Consequences of Abstinence and Alcobol Use in Young Adulthood}

Recent studies have suggested that drinking may have some beneficial functions for adolescents in terms of personal and social development. The general picture is that abstainers are less socially integrated, are less actively engaged in romantic affairs and are less inclined to adopt adult roles compared to (moderate) drinkers (see Engels \& Knibbe, 1998b). The evidence for enhanced levels of distress, low selfesteem or depression in abstainers is less clear. Our studies suggest that many of the social effects attributed to drinking might be, in fact, explained by the situation in which youngsters consume alcohol, namely pubs, discos and parties. This issue is also raised in chapter 7 where the focus is on the relationship between distress and alcohol consumption in male 'home drinkers'. Some of the problems described earlier in this chapter are taken care of in this proposed project.

First, a major limitation of our study points to the cross-sectional amalyses. We do know something about the functions of drinking in late adolescence. Nonetheless, it is possible that the majority of the abstainers become well adjusted persons in young adulthood. On the other hand, adolescent abstainers might get problems within family relationships or with peer relationships later on. A prospective study which follows late adolescents into young adulthood might solve this important problem of generalization.

Second, our research on functions of alcohol use is restricted primarily to positive aspects of drinking. Of course, adolescent drinking will have negative consequences and abstinence will have some positive consequences. It is important to investigate which elements of positive and negative consequences are interrelated and how they are linked to individual drinking preferences. The perceptions of consequences and expectancies about occurrence of these consequences might be helpful in studying two questions. First, most youngsters go to public drinking places to have a good time and to drink. However, for some of them drinking results in aggression, sexual harassment, problems with relationships with friends and partner, drunk driving, problem drinking etcetera. Why some youngsters go over the edge is unknown. Information about positive and negative consequences and expectations of different categories of drinkers could be a starting point. Second, it is well known that for some people, alcohol functions as a coping mechanism to reduce feelings of distress. Our findings on the context of drinking 
suggests that certain categories of youngsters are more inclined to adopt negative drinking styles. In particular, longitudinal research can sort out whether drinking as a coping mechanism in adolescence leads to more problematic drinking and social functioning in young adulthood.

Another limitation of the present study is its quantitative nature. Understanding of functions of drinking will improve if, besides standardized questionnaires, in-depth interviews are also conducted. In this way, theoretical notions can be tested in interviews with representatives of different categories (differing on drinking levels and drinking context).

In the proposed study, it is planned that 800 male respondents in the 17-19 age category participate. This sample will consist of three categories namely abstainers, moderate drinkers and heavy drinkers $(n=200,400,200$, respectively). Thus, the sample is not meant to be representative, but chosen to assure variation in theoretically relevant variables. Respondents will be approached by means of advertisements in local and national newspapers, in schools, in public drinking places by Consultation Bureaus for Alcohol and Drug Problems (CAD) and municipal health services (GGD). After a screening questionnaire, a written questionnaire will be sent to the respondents. Additional semi-structured interviews will be held with 15 subjects from each category. A second wave (questionnaires and interviews) will be conducted four years later. This interval is chosen because at the age of 21-23, most people have entered other stages of life in comparison to late adolescence, such as living on their own, following post high school education or working, or starting cohabiting or marrying.

\section{Predictors of Stage Transitions in Adolescent Smoking Cessation}

Smoking cessation among youngsters is largely neglected by researchers and in practice (see chapter 8 ). It is regrettable that the majority of prevention programs focus on smoking onset while prevalence figures show that many youngsters become regular smokers despite all the efforts. To decrease regular smoking, attention should be paid to quitting. In our study on the prospective value of smoking-specific cognitions, environmental smoking and habitual factors on youngsters' motivation to quit, we gained insight into the quitting process. However, our study suffered from some shortcomings.

Although a longitudinal study was used to examine predictors of subjects' motivation to quit, a design with extended intervals between the waves might not provide an adequate view of the dynamics of the quitting process. In particular, because some studies have shown that individuals' opinions, ideas, and intentions about quitting might vary even over a few months, it is desirable to monitor a group of young smokers for some time. Additionally, we did not investigate the validity of the stage approach employed. Whether people go through stages of change gradually can only be tested in a longitudinal design. Furthermore, it is 
likely that the precursors of the transition from precontemplation to contemplation are different from the transition from preparation to actual quitting (e.g., De Vries \& Backbier, 1996; Dijkstra et al., 1996). We could not test this assumption in our study. If different processes and factors are involved in different stages of smoking cessation, this would add to the theoretical and practical value of this model.

Smoking attitudes, self-efficacy expectations and smoking behavior in midadolescence predicted subjecrs' motivation to quit in late adolescence. Nonetheless, smoking behavior of others and perceived pressure to smoke were not related to quitting. To explore this latter finding in more detail, adolescents' parents and best friends should be asked to participate in a study. In that way, more knowledge on facilitating aspects as well as social barriers in the quitting process can be gained.

The research questions will be addressed in an 18 months 4 waves prospective study. 500 young smokers (14-16-year olds) in different stages of onset and quitting will be included. All subjects will fill out questionnaires. Qualitative in-depth interviews will be held with 40 subjects at each wave (differing on stage-allocation at the first wave). Additionally, (one) parent(s) and a best friend of these 40 subjects will fill out a specific questionnaire. Secondary schools (vocational schools and high schools) will be approached to trace the smokers, and administration of the questionnaires will be carried out at school. The questionnaires will include scales on stage-allocation, history and actual smoking behavior, self-efficacy, pros and cons of smoking and quitting, motivation to quit, processes of change, social support, family and peer smoking. The questionnaires for referent persons will include scales on their own smoking behavior, adolescent smoking behavior, smoking norms, pressures to quit and smoke, and social support during a quit attempt.

\section{NOTES}

${ }^{1}$ Because in these studies, smoking by referent others was assessed by asking respondents to provide information, their responses could not only reflect the actual behavior of others but also subjects' beliefs, norms and interpretation. Strictly speaking, modelling factors can be seen as cognitions (see Graham et al., 1991)

2 'The descriptions are very brief and are meant to give an impression of the projacts. We have worked out the first two projects in more detail. 



\section{REFERENCES}

Abrams, D., Follick, M., Biener, I., Carey, K., \& Hitti, J. (1997). Saliva cotinine as a measure of smoking status in field settings. American Joumal of Public Health, 77, 846-848.

Abrams, D. B., \& Niaura, R. S. (1987). Social Learning Theory. In H.T. Blane \& K.E. Leonard (Eds.), Psychological theories of drinking and alcobolism ( $\mathrm{pp}, 131-178)$. New York: Guilford Press.

Ahlgren, A., Norem, A. A., Hochhauser, M. \& Gravin, J. (1982). Antecedents of smoking among pre-adolescents. Journal of Drag Education, 12, 325-340.

Ajzen, I. (1985). From intentions to actions: A theory of planned behavior. In J. Kuhl \& J. Beckmann (Eds). Action-control: From cognition to bebavior (pp. 11-39). Heidelberg: Springer.

Ajzen, 1. (1991). The theory of planned behavior. Onganizational Bebatior and Human Decision Processes, 50, 179-211.

Ajzen, I., \& Fishbein, M. (1980). Understanding atitudes and predicting social bebavior. Englewood Cliffs, NJ: Prentice-Hall.

Akers, R. L. (1977). Deviant behavior: A social leaming approach, 2nd ed. Bellmont: Wadsworth.

Akers, R. L., Krohn, M. D., Lanza-Kaduce, L., \& Radosevich, M. (1979). Social learning and deviant behavior: A specific test of a general theory. American Sociological Review, 44, 636-655.

Akers, R. L., Massey, J., Clarke, W., 8 Lauer, R. M. (1983). Are self-reports of adollescent deviance valid? Biochemical measures, randomized tesponses and the bogus pipeline in smoking behavior. Social Forces, 62, 234-251.

Allegrante, J. P., O'Rourke, T. W., \& Tuncalp, S. (1977). A multwariate analysis of selected psychosocial variables in the development of subsequent youth smoking foumal of Drug Education, 7, 237-248. 
Aloise, P. A., Graham, J. W., \& Hansen, W. B. (1994). Peer influence on smoking initiation during early adolescence: A comparison of group members and group outsiders. Journall of Applied Psychology, 79, 281-287.

Ary, D. V., \& Biglan, A. (1988). Longitudinal changes in adolescent cigarette smoking behavior: Onset and cessation. fowmal of Bebavioral Medicine, 11, 361-382.

Aseltine, R. H. (1995). A reconsideration of parental and peer influences on adolescent deviance. Journal of Health and Social Behavior, 36, 103-121.

Bach, P. J., \& Schaefer, J. M. (1979). The tempo of country music and rate of drinking in bars. Joumal of Studies on Alcohol, 40, 1058-1059.

Bagnall, G. (1988). Use of alcohol, tobacco and illicit drugs amongst 13-year-olds in three areas of Britain. Drug and Alcobol Dependence, 22, 241-251.

Bagnall, G., \& Plant, M. A. (1987). Adolescent drinking. British journal of Addiction, 82, 829830.

Bailey, S. L. (1992). Adolescents' multisubstance use patterns: The role of heavy alcohol and cigarette use. American Joumal of Public Health, 82, 1220-1124.

Bailey, S. L., Flewelling, R. L., \& Rachal, J. V. (1992). The characterization of inconsistencies in self-reports of alcohol and marijuana use in a longitudinal study of adolescents. Journal of Studies an Alcohol, 53, 636-647.

Bailey, S., \& Hubbard, R. L. (1990). Developmental variation in the context of marijuana initiation among adolescents. Journal of Health and Social Behavior, 31, 58-70.

Bandura, A. (1977). Social learning theory. Englewood Cliffs: Prentice-Hall.

Bandura, A. (1986). Social foundations of thought and action: A social cognitive theory. New York: Prentice-Hall.

Barnea, Z., Rahav, G., \& Teichman, M. (1987). The reliability and consistency of self-reports on substance use in a longitudinal study. British Joumal of Addiction, 82, 891-898.

Bauman, K. E., \& Ennett, S. T. (1996). On the importance of peer influence for adolescent drug use: Commonly neglected considerations. Addiction, 91, 185-198.

Bauman, K. E., Fisher, L. A., \& Koch, G. G. (1989). External variables, subjective expected utility, and adolescent behavior with alcohol and cigarettes. Journal of Applied Social Psychology, 19, 789-804.

Baumrind, D. (1985). Familial antecedents of adolescent drug use: A developmental perspective. In C. L. Jones \& R. J, Battjes (Eds.), Etiology of drug abuse: Implications for prevention (pp. 13-44). Rockville, MD: National Institute of Drug Abuse.

Beck, K. H., Thombs, D. L., \& Summons, T. G. (1993). The social context of drinking scales: Construct validation and relationships to indicants of abuse in an adolescent population. Addictive Behaviors, 18, 159-169.

Bem, D. J. (1972). Self-perception theory. In: L. Berkowitz (Ed.), Advances in experimental social psychology (pp. 1-62). New York: Academic Press.

Bentler, P. M., \& Speckart, G. (1979). Models of attitude-behavior relations. Psychological Review, 86, 452-464.

Berndt, T. J. (1982). The features and effects of friendship in early adolescence. Cbild Development, 53, 1447-1460.

Biddle, B. J., Bank, B. J., \& Marlin, M. M. (1980). Parental and peer influence on adolescents. Social Forces, $58,157-179$.

Bien, T. H., \& Burge, R. (1990). Smoking and drinking: A review of the literature. International Joumal of the Addictions, 25, 1429-1454.

Biglan, A., Severson, H., Bavry, J., \& McConnell, S. (1983). Social influence and adolescent smoking: A first look behind the barn. Healtb Education, 14-18. 
Billy, J. O. G., \& Udry, J. R. (1985). Patterns of adolescent friendship and effects on sexual behavior. Social Psychology Quarterly, 48, 27-41.

Bollen, K. A. (1989). Structural equations with latent variables. New York: Wiley.

Botvin, G. J., \& Botvin, E. M. (1992). Adolescents tobacco, alcohol, and drug use: Prevention strategies, empirical findings, and assessment issues. Developmental Behavioral Pediatrics, 13, 290-301.

Botvin, G. J., Eng, A., \& Williams, C. L. (1980). Preventing the onset of cigarette smoking through life skills training. Preventive Medicine, 11, 199-211.

Botvin, G. J., Schinke, S. P., \& Orlandi, M. S. (1989). Psychosocial approaches to substance abuse prevention: Theoretical foundations and empirical findings. Crisis: International Joumal of Suicide and Crisis Studies, 10, 62-77.

Breslau, N., Kilbey, M. M., \& Andreski, P. (1994). DSM-III-R nicotine dependence in young adults: Prevalence, correlates and associated psychiatric disorders. Addiction, 89, 743754.

Breslau, N., \& Peterson, E. L. (1996). Smoking cessation in young adults: Age at initiation of cigarette smoking and other suspected influences. American Joumal of Public Health, 86, 214-220.

Brouwer, M. B. D., \& Kingma, J. (1995). Trends in fetsongevallen: Drastische toename varz alcobolgebruik bij jongeren (Trends in bicycle accidents: Dramatic increase in alcohal wse among adolescents). Acadernisch Ziekenhuis Groningen.

Brown, B. B. (1990). Peer groups and peer cultures. In S. S. Feldman \& G. R. Elliott (Eds.), At the threshold: The developing adolescent. Cambridge: Harvard University Press.

Brown, S. A., Creamer, V. A., \& Stetson, B. A. (1987). Adolescent alcohol expectancies in relation to personal and parental drinking patterns. Joumal of Abnormal Psychology, 96, $117-121$.

Bruwold, W. H. (1993). A meta-analysis of adolescent smoking prevention programs. American Joumal of Public Health, 83, 872-880.

Buisman, W. R., \& Esseveld, P. (1988). Alcoholpreventie onder jongeren: De rol van opwoeding en onderwijs (Alcohol prevention for adolescents: The role of child-rearing and education). In J. C. Van der Stel \& W. R. Buisman (Eds.), Alcoholpreventie: Achtergronden, praktijk en beleid (pp. 148-16́5). Alphen aan den Rijn: Samson.

Burton, D. (1994). Tobacco cessation programs for adolescents. In R. Richmond (Ed.), Interventions for smokers: An international perspective. Baltimore: Williams and Wilkens.

Charlton, A. (1984). Smoking cessation help for young people: The process observed in two case studies. Health Education Research, 7, 249-257.

Charlton, A., \& Blair, V. (1989). Predicting the onset of smoking in boys and girls. Social Science and Medicine, 29, 813-818.

Chassin, L., Corty, E., Presson, C. C., Olshavsky, R. W., Bensenberg, M., \& Sherman, S. J. (1981). Predicting adolescents' intentions to smoke cigarettes. Jowrnal of Health and Social Behavior, 22, 445-455.

Chassin, L., Presson, C. C., Rose, J. S., \& Sherman, S. J. (1996). The natural history of cigarette smoking from adolescence to adulthood: Demographic predictors of continuity and change. Health Psychology, 15, 478-484.

Chassin, L., Presson, C. C., \& Sherman, S. J. (1984). Cognitive and social influence factors in adolescent smoking cessation. Addictive Behaviors, 9, 383-390.

Chassin, L., Pressan, C. C., Sherman, S. J., Corty, E., \& Olshavsky, R. W. (1984). Predicring the onset of cigarette smoking in adolescents: A longitudinal study. Joumal of Applied Social Psychology, 14, 224-243. 
Chassin, L., Presson, C. C., Sherman, S. J. \& Edwards, D. A. (1990). The naturall history of cigarette smoking: Predicting young-adult smoking outcomes from adolescent smoking parterns. Health Psychology, 9,701-716.

Chassin, L., Presson, C. C., Sherman, S. J., \& Edwards, D. A. (1992). The natural history of cigarette smoking and young adult social roles. Joumal of Health and Social Bebavior, 33, 328-347.

Chassin, L., Presson, C. C., Sherman, S. J., Montello, D., \& McGrew, J. (1986). Changes in peer and parent influence during adolescence: Longitudinal versus cross-sectional perspectives on smoking initiation. Developmental Pycbology, 22, 327-234.

Christiansen, B. A., \& Goldman, M. S. (1983). Alcohol-related expectancies versus demographic/ background variables in the prediction of adolescent drinking foumal of Consulting and Clinical Psychalogy, 51, 249-258.

Christiansen, B. A., Smith, G. T., Roehling, P. V., \& Goldman, M. S. (1989). Using alcohol expectancies to predict adolescent drinking behavior after one year. Joumal of Corsulting and Clinical Psychology, 57, 93-99.

Cialdini, R. B., Reno, R. R., \& Kallgren, C. A. (1990). A focus theory of normative conduct: Recycling the concept of norms to reduce littering in public places. Joumal of Personality and Social Psychology, 58, 1015-1026.

Cohen, J. M. (1977). Sources of peer group homogeneity. Sociology of Education, 50, 227-241.

Cohen, S., Kamarck, T., \& Mermelstein, R. (1983). A global measure of perceived stress. Joumal of Health and Social Behavior, 24, 385-396.

Cohen, $S_{4}$, \& Lichtenstein, E. (1990). Partner behaviors that support quitting smoking. Joumal of Consuling and Clinical Psychology, 58, 304-309.

Collins, L. M., Sussman, S., Mestel Rauch, J., Dent, C. W., Anderson Johnson, C., Hansen, W. B., \& Flay, B. R. (1987). Psychosocial predictors of young adolescent cigarette smoking: A sixteen-month three-wave longitudinal study. Jowmal of Applied Social Psychology, 17, 554.573.

Conger, J. J. (1956). Alcoholism: Theory, problem and challenge. II. Reinforcement theory and the dynamic of alcoholism. Quarterly Joumal of Studies on Alcohol, 13, 296-305.

Conrad, K. M., Flay, B. R., \& Hill, D. (1992). Why children start smoking cigarettes: Predictors of onset. Britisb Joumal of Addiction, 87, 1711-1724.

Cooper, M. L., Russell, M., \& George, W. H. (1988). Coping, expectancies, and alcohol abuse: A test of social learning tormulations. Joumal of A bnomal Psychology, 97, 218-230.

Cooper, M. L., Russell, M., Skinner, J. B., Frone, M. R., \& Mudar, P. (1992). Stress and alcohol use: Moderating effects of gender, coping, and alcohol expectancies. fowmal of Abnormal Psycbology, 101, 139-152.

Cooper, M. L., Russell, M., Skinner, J. B., \& Windle, M. (1992). Development and validation of a three-dimensional measure of drinking motives. Psychological Assessment, 4, 123132.

Cooperstock, R., \& Parnell, P. (1982). Research on psychotropic drug use: A review of findings and methods. Social Science and Medicine, 16, 1179-1196.

Cutler, R. E., \& Storm, T. (1975). An observational study of alcohol consumption in natural settings: The Vancouver Beer Parlor. Jowmal of Studies on Alcobol, 36, 1173-1183.

Czarnecki, D. M., Russell, M., Cooper, M. L., \& Salter, D. (1990). Five-year reliability of selfreported alcohol consumption. foumal of Stwdies on Alcohol, 51, 68-76.

Darby, W. (1983). To optimize use of alcoholl beverages. In: R. J. J. Hermus (Ed.), Proceedings of the symposinm Alcohol, Healith and Society (pp. 109-124). Zeist: TNO. 
Dekovic, M., \& Janssens, J. M. (1992). Parents' child-rearing style and child's sociometric status. Developmental Pychology, 28, $925-932$.

DeMaris, A. (1995). A tutorial in logistic regression. Joumal of Marriage and the Family 57,956 968.

De Vries, H. (1989). Smoking prevention in Dutch adolescents. Dissertation. Mastricht: Datawyse.

De Vries, H., \& Backbier, $\mathbb{E}$. (1994). Self-efficacy as an important determinant of quitting among pregnant women who smoke: The p pattern. Preventive Medicine, 23, 167.174.

De Vries, H., Backbier, E $E_{n,}$ Kok, G., \& Dijkstra, M. (1995). The impact of social influences in the context of attitude, self-efficacy, intention and previous behavior as predictors of smoking onset. Joumal of Applied Social Pychology, 25, 237-257.

De Vries, H., Dijkstra, M., \& Kuhlman, P. (1988). Self-efficacy: The third factor besides attitude and subjective norm as a predictor of behavioral intentions. Health Education Research, 3, 273-282.

De Vries, H., \& Kok, G. J. (1986). From determinants of smoking belnaviour to the implications for a prevention programme. Heath Education Research, 1, 85-94.

De Zwart, H., Zwart, H., \& Kuipers, S. B. M. (1997). Kenngegevens: Roken, drinken, drwggebruik en gokken onder scbolieren wanaf 10 jaar. Urrecht: Trimbos-instituut.

De Zwart, R., Warnaar, M. F., \& Van Dam, B. M. T. M. (1994). Scholievenonderzoek 1994 (Reports on secondary schoolchildren 1994). Den Haag: NIBUD.

DiClemente, C. C., Prochaska, J. O., Fairhurst, S. K., Velicer, W. F", Velasquez, M. M., \& Rossi, J. S. (1991). The process of smoking cessation: Precontemplation, contemplation and preparation stages of change. Jowmal of Consulting and Clinical Psychology, 59, 295. 304.

Dijkstra, A. (1998). Computer-tailored interventions for smoking cessation. Targeting smokers with low readiness to quit. Dissertation. Maastricht: Datawyse.

Dijkstra, A., De Vries, H., \& Bakker, M. (1996). Pros and cons of quitting, self-efficacy, and the stages of change in smoking cessation. Journal of Consulting and Clinical Psychology, 64, 758-763.

Dolcini, M. M., \& Adler, N. E. (1994). Perceived competencies, peer group affiliation, and risk behavior among adolescents. Health Psychology, 13, 496-506.

Donovan, J. E., \& Jessor, R. (1985). Structure of problem behavior in adolescence and young adulthood. Joumal of Consulting and Cinical Psychology, 53,890-904.

Doreian, P. (1986). Measuring relative standing in small groups and bounded social networks. Social Psychology Quarterly, 49, 247-259.

Douvan, E., \& Adelson, J. (1966). The adolescent experience. New York: Wiley.

Dusenbury, L., Epstein, J. A., Botvin, G. J., \& Diaz, T. (1994). Social influence predictors of alcohol use among New York Latino youth. Addictive Bebavions, $19{ }_{n} 363-372$.

Dutch Foundation on Smoking and Health (1996). Jaarverslag 1995 (Anwal report 1995). The Hague.

Eagly, A. H., \& Chaiken, S. (1993). The psychology of attitudes. Forth Worth, TX: Harcourt Brace Jovanovich.

Eggert, L. L., \& Herting, J. R. (1993). Drug involvement among potential dropouts and "typical" youth. Journal of Drug Education, 23, 31-55.

Eiser, J. R., Morgan, M., Gammage, P., Brook, N., \& Kirby, R. (1991). Adolescent health behaviour and similarity-attraction: Friends share smoking habits (really), but much else besides. British Journal of Social Pychology, 30, 339-3.48. 
Eiser, J. R. Van Der Pligt, J., Raw, M., \& Sutton, S. R. (1985). Trying to stop smoking: Effects of perceived addiction, attributions to failure, and expectancy of success. Jowmal of Behavioral Medicine, 8, 321-341.

Elliotr, D. S., Huizinga, D., \& Ageton, S. S. (1985). Explatining delinquency and drug wse. New York: Springer-Verlag.

Endler, N. S., 82 Parker, D. A. (1994). Assessment of multidimensional coping: Task, emotion and avoidance strategies. Psycbological Assessment, 6, 50-60.

Engels, R. C. M. E., \& Knibbe, R. A. (1997a). De zonnige zijde van alcoholgebruik. De betekenis van drinken en uitgaan voor jongeren (The bright side of alcohol use: The meaning of drinking and pub-going for adolescents). Tijdschrift voor Alcabol, Drugs en andere Psychotrope Stoffen, 22, 154-163.

Engels, R. C. M. E., \& Knibbe, R. A. (1997b). Dilemma's bij bet meten van sociale beinvloedingsprocessen (Dilemmas with the assessment of social influchce processes) Paper gepresenteerd bij het $12^{\text {de }}$ symposium van de Associatie van Social Psychologische Onderzoekers, Universiteit Twente.

Engels, R. C. M. E., \& Knibbe, R. A. (1998). Alcohol use and intimate relationships in adolescence: When love comes to town. Manuscript submitted for publication.

Engels, R. C. M. E., Knibbe, R. A., De Vries, H., \& Drop, M. J. (1998). Antecedents of smoking cessation among adolescents: Who is motivated to change? Preventive Medicine, 27, 348-357.

Engels, R. C. M. E., Knibbe, R. A., De Vries, H., Drop, M. J., \& Van Breukelen, G. J. P. (1998). Influences of parental and best friends" smoking and drinking on adolescent use: A longitudinal study. Joumal of Applied Social Psychology (in press).

Engels, R. C. M. E., Knibbe, R. A., \& Drop, M. J. (1996). Facilitating effects of alcobol consumption: Related to the sitwation? Paper presented at the $22^{\text {nd }}$ Annual Symposium of the Kettil Bruun Society on Social and Epidemiological Research on Alcohol, Edinburgh.

Engels, R. C. M. E., Knibbe, R. A., \& Drop, M. J. (1997a). Wie beginnen er met roken? Een kritische studie naar de voorspelbaarheid van rookgedrag bij jongeren (Who starts to smoke? A critical study on the predictability of smoking onset of adolescents). In D. Daamen, A. Pruyn, W. Otren \& R. Meertens (Eds.), Sociale Psychologie en baar roepassingen (pp. 159-171). Eburon: Delft.

Engels, R. C. M. E., Knibbe, R. A., \& Drop, M. J. (1997b). Inconsistencies in adolescents" selfreports of initiation of alcohol and tobacco use. Addictive Bebaviors, 22, 613-623.

Engels, R. C. M. E., Knibbe, R. A., \& Drop, M. J. (1998a). Visiting public drinking places: An explorative study into the functions of pub-going for late adolescents. Accepted for publication in Substance Use and Mistuse.

Engels, R. C. M. E., Knibbe, R. A., \& Drop, M. J. (1998b). Predictability of smoking in adolescence: Between optimism and pessimism. Accepted for publication in Addiction.

Engels, R. C. M. E., Knibbe, R. A., \& Drop, M. J. (1998c). Changes in smoking and drinking in secondary schoolchildren: A three wave five year longitudinal study. Manuscript submitted for publication.

Engels, R. C. M. E., Knibbe, R. A., \& Drop, M. J. (1998d). Why do late adolescents drink at home? A study on psychological well-being, social integration and drinking context. Accepted for publication in Addiction Research.

Engels, R. C. M. E., Knibbe, R. A., Drop, M. J., \& De Haan, J. T. (1996). Invloed en selectie in vriendschapsrelaties van adolescenten: Een longitudinale studie naar homogeniteit in 
rookgedrag (Influence and selection in friendships of adolescents: A longitudinal study on homogeneity in cigarette smoking). Gedrag en Gezondheid, 23, 257-268.

Engels, R. C. M. E., Knibbe, R. A., Drop, M. J., \& De Haan, J. T. (1997). Homogeneity of smoking behawior in peer groups: Influence or selection? Health Edsucation and Bebavior, 24, 801-811.

Ennett, S. T., \& Bauman, K. E. (1993). Peer group structure and adolescent cigarette smoking: A social network analysis. Journal of Health and Social Behawior, 34, 226-236.

Ennett, S. T., \& Bauman, K. E. (1994). The contribution of influence and selection to adolescent peer group homogeneity: The case of adolescent cigarette smoking. foumal of Personality and Social Psychology, 67, 653-663.

Epstein, S. (1979). The stability of behavior: On predicting what most people do much of the time. Joumal of Personality and Social Psycbology, 37, 1097-1126.

Erikson, E. (1958). Identity: Youth and crisis. New York: W. W. Norton \& Company.

Ershler, J., Leventhal, H., Fleming, R., \& Glynn, K. (1989). The quitting experience for smokers in sixth through twelfth grades. Addictive Behaviors, 14, 365-378.

European Bureau for Action on Smoking Prevention (1994). Tobacco atd health: An overvien. Bruxelles.

Evans, R. I., Dratt, L. M., Raines, B. E., \& Rosenberg, S. S. (1988). Social influences on smoking initiation: Importance of distinguishing descriptive versus mediating process variables. Joumal of Applied Social Psychology, 18, 925-943.

Evans, R. I., Hanson, W. B., \& Mittelmark, M. B. (1977). Increasing the validity of self-reports of smoking behaviour in children. Jowmal of Applied Psychology, 62, 521-532.

Evans, R. I., Rozelle, R. M., Mittelmark, M. B., Hansen, W. B., Bane, A. L., \& Havis, J. (1978). Deterring the onset of smoking in children: Knowledge of immediate physiological effects and coping with peer pressure, media pressure and parent modeling. Jowrnal of Applied Social Psychology, 8, 126-135.

Fagerstrom, K. O. (1991). Towards better diagnoses and more individual treatment of tobacco dependence. British Jourmal of Addiction, 86, 543-547.

Farell, A. D. (1994). Structural equation modeling with longitudinal data: Strategies for examining group differences and reciprocal relationships. Joumal of Consulting and Clinical Psychology, 62, 477-487.

Farkas, A. J., Pierce, J. P., Zhu, S. H., Rosbrook, B., Gilpin, E. A., Berry, C. et al. (1996) Addiction versus stages of change models in predicting smoking cessation. Addiction, $91,1271-1280$.

Fava, J.L., Velicer, W.F., \& Prochaska, J.O. (1995). Applying the transtheoretical model to a representative sample of smokers. Addictive Bebaviors, 20, 189-203.

Fazio, R. H. (1986). How attitudes guide behavior? Handbook of motivation and cogntion: Foundations of social bebawior (pp. 204-243). New York: Guilford Press.

Feinhandler, S. J. (1986). "The social role of smoking. In R. D. Tollison (Ed.), Smoking and society: Towards a more balanced assessment (pp. 167-187). Lexington, Massachusetts:

D.C. Heath and Company.

Fergusson, D. M., Lynskey, M. T., \& Horwood, J. L. (1995). The role of peer affiliations, social, family and individual factors in continuicies in cigarette smoking between childhood and adolescence. Addiction, 90,647-659.

Festinger, L. (1957). A theory of cognitive dissonance. New York: Row and Peterson.

Fillmore, K. M. (1988). Alcobol wise across the life course: A critical revien of the 70 years of longitudinal resedrch. Toronto: ARF. 
Fillmore, K. M. (1996). Selected contributions from alcobol-related longitudinal research. Paper presented at the $22^{\text {nd }}$ Annual Alcohol Epidemiology Symposium of the Ketril Bruun Society, Edinburgh, Scotland.

Fishbein, M., \& Ajzen. I. (1975). Belief, attitsude, intention and bebavior: An introdsction to theory and research. Reading (MA): Addison-Wesley.

Fisher, L. A., \& Bauman, K. E. (1988). Influence and selection in the friend-adolescent relationship: Findings from studies of adolescent smoking and drinking. Joumal of Applied Social Psychology, 18, 289-314.

Flay, B. R. (1985). Psychosocial approaches to smoking prevention: a review of findings. Health Psychology, 4, 449-488.

Flay, B. R, d'Avernas, J., Best, J. A., Kersell, M. W., \& Ryan, K. B. (1983). Cigarette smoking: Why young people do it and ways of prewenting it. In P. J. McGrath \& P. Firestone (Eds.), Pediatric and Adolescent Bebawional Medicine (pp. 131-183). New York: SpringerVerllag.

Flay, B. R., Hu, F. B., Siddiqui, O., Day, L., E., Hedeker, D., Petraitis, J., Richardson, J., \& Sussman, S. (1994). Differential influence of parental smoking and friends" smoking on adolescent initiation and escalation of smoking. Joumal of Health and Social Bebavior, $35,248 \times 265$.

Filay, B. R., \& Petraitis, J. (1994). The theory of triadic influence: A new theory of health behavior with implications for preventive interventions. In G. Albrecht (Ed), Advances in Medical Sociology. Vol 4. A reconsideration of health bebavior cbange models (Pp. 19-44). Greenwich: JAI Press.

Flemming, J. P., Kellam, S.G., \& Brown, C. H. (1982). Early predictors of age at first use of alcohol, marijuana, and cigarettes. Drug and Alcobol Dependence, 9, 285-303.

Folkman, S., \& Lazarus, R. S. (1988). Coping as a mediator of emotion. Joumal of Personality and Social Psycbology, 54, 466-475.

Forsyth, D. R. (1990). Group dynamics. Pacific Grove CA: Brooks/Cole Publishing Co.

Friedman $_{n}$ L. S., Lichtenstein, E., \& Biglan, A. (1985). Smoking onset among teens: An empirical analysis of initial situations. Addictive Bebaviors, 10, 1-13.

Gagne, M. H., \& Lavoie, F. (1993). Young people's views on the causes of viollence in adolescents' romantic relationships. Carwada's Merital Health, 41, 11-15.

Glynn, T. J. (1989). Essential elements of school-based smoking prevention programs. Jowmal of School Health, 59, 181-188.

Glynn, K., Leventhal, H., \& Hirschman, R. (1985). A cognitive development approach to smoking prevention. In C. S. Bell \& R. Battjes (Eds.), Prevention researcb: Deterring drug abuse among children and adolescents. NIDA Research Monograph 63. Washington, DC: U.S. Government Printing Office.

Goddard, E. (1990). Wby cbildren start smoking. London: HMSO.

Gordon, N. P. (1986). Never smokers, triers and current smokers: Three distinct target groups for school-based antismoking programs. Health Education Quarterly, 13,163-180.

Graham, J. W., Marks, G., \& Hansen, W. B. (1991). Social influence processes affecting adolescent substance use. foumal of Applied Psychology, 76, 291-298.

Grant, B. F., Marford, T. C., \& Grigson, M. B. (1988). Stability of alcohol consumption among youth: A national longitudinal study. fonmal of Studies on Alcohol, 49, 253-260.

Green, G., Macintyre, S., West, P., \& Ecob, R. (1991). Like parent like child? Associations between drinking and smoking behaviour of parents and their children. British Jommal of Addiction, $86,745-758$. 
Grube, J. W., Rokeach, M., \& Getzlaf, S. B. (1990). Adolescents' value images of smokers, ex-smokers, and nonsmokers. Addictive Behaviors, 15, 81-88.

Guy, S. M., Smith, G. M., \& Bentler, P. M. (1994). Consequences of adolescent drug use and personality factors on adult drug use. Joumal of Drug Education, 24, 109-132.

Hajema, K. J. (1998). Sociological aspects of drinking behavior, alcobol-related problems and belp seeking. Dissertation. Maastricht: Datawyse.

Hanna, E. Z., Faden, V. B. \& Harford, T. C. (1993). Marriage: Does it protect young women trom alcoholism? Joumal of Substance Abuse, $5,1-14$.

Hansen, W. B. (1983). Behavioral predictors of abstinence: Early indicators of a dependence on tobacco among adolescents. Intemational Joumal of the Addictions, 18, 413-420.

Hansen, W. B., Collins, L. M., Johnson, C. A., \& Graham, J. W. (1985). Self-initiated smoking cessation among high school students. Addictive Behwviors, 10, 265-271.

Hansen, W. B., Graham, J. W., Sobell, J. L., Shelton, D. R., Flay, B. R., \& Anderson Johnson, C. (1987). The consistency of peer and parent influences on tobacco, alcohol, and marijuana use among young adolescents. Journal of Bebavioral Medicine, 10, 559-579.

Harford, T. C., \& Grant, B. F. (1987). Psychosocial factors in adolescent drimking contexts. Journal of Studies on Alcobol, 48, 551-557.

Hartup, W. W. (1983). Peer relations. In E. M. Hetherington (Ed.), Handbook of cbild psycbology. Vol IV. Socialization, personality and social development (pp. 124-196). New York: Wiley.

Hewett, P. L., Flett, G. L., \& Mosher, S. W. (1992). The Perceived Stress Scale: Factor structure and relation to depression symptoms in a psychiatric sample. Joumal of Psychopathology and Behavioral Assessment, 14, 247-257.

Hingson, R., Heeren, T., Howland, J., \& Winter, M. (1991). Reduced BAC limits for young people. Alcohol, Drugs and Driwing, 7, 117-127.

Hirschi, T. (1969). Causes of delinquency. Berkeley CA: University of California Press.

Hirschman, R. S., Leventhal, H., \& Glynn, K. (1984). The development of smoking behavior: Conceptualization and supportive cross-sectional survey data. Journal of Applied Social Psycbology, 14, 184-206.

Hoffman, J. A. (1984). Psychological separation of late adolescents from their parents. Jowmal of Counselling Psychology, 31, 170-178.

Hosmer, D. W., \& Lemeshow, S. (1989). Applied logistic regression. New York: John Wiley \& Sons.

Hu, F. B., Flay, B. R., Hedeker, D., Siddiqui, O., \& Day, L. E. (1995). The influences of friends" and parental smoking on adolescent smoking behavior: "The effects of time and prior smoking. Journal of Applied Sacial Psychology, 25, 2018-2047.

Iversen, E. (1996). Social activivy, social norms and alcohol consumption among older adolescents and young adults in Western Nortacy. Paper presented at the $22^{\text {nd }}$ Annual Alcohol Epidemiology Symposium of the Ketti] Bruun Society, Edinburgh, Scotland.

Jarvis, M. J., Goddard, E., \& McNeill, A. (1990). Do attitudes predict uptake of smoking in teenagers? Case not prowen. Social Science and Medicine, 31, 997-1001.

Jenson, E. J., \& Overgaard, E. (1993). Investigation of smoking habits among 14-17-year-old boarding school pupils: Factors which influence smoking status. Public Health, 107, 117 . 123.

Jessor, R. (1987). Problem-Behavior Theory, psychosocial development, and adolescent problem drinking. British Joumal of Addiction, 82, 331-342.

Jessor, R. (1991). Risk behavior in adolescence: A psychosocial framework for understanding and action. Joumal of Adolescent Health, 12,597-605. 
Jessor, R., \& Jessor, S. L. (1977). Problem behavion and prychosocial development: A longitudinal study of youth. New York: Academic Press.

Johnson, D. W. (1980). Constructive peer relationships, social development and cooperative learning experiences: Implications for the prevention of drug abuse. Jowmal of Drag Education, 10, 7-24.

Johnson, K. A., \& Jennison, K. M. (1992). The drinking-smoking syndrome and social context. International Joumal of the Addictions, 27, 749-792.

Johnson, V. (1988). Adolescent alcohol and marijuana use: A longitudinal assessment of a social learning perspective. American Joumal on Drug and Alcohol Abuse, 14, 419-439.

Johnston, L. D., O'Malley, P. M. \& Bachman, J. G. (1991). Drug use among American High School Seniors, College Students and Young Adults, 1975-1990: Volume 1 High School Seniors (pp. 27-33) U.S. Department of Health and Human Services , DHHS Publication No. (ADM) 91-113. Washington, DC: U.S. Government Printing Office.

Johnston, L. D., O'Malley, P. M., \& Bachman, J. G. (1996). National survey results on drug use from the Monitoring the Future study, 1975-1995. Volume I: Secondary school students. (NIH Pub. No. 97-4139). Rockville, MD: National Institute on Drug Abuse.

Jöreskog, K. G., \& Sörbom, D. (1989). LISREL 7: A guide to the program and applications. Chicago: SPSS Inc.

Jöreskog, K. G., 8x Sörbom, D. (1993). LISREL 8. Structural equation modeling with the SIMPLIS command language. Hillsdale (NJ): Erlbaum, SSI Scientific Software Int.

Jussim, L., \& Osgood, D. W. (1989). Influence and similarity among friends: An integrative model applied to incarcerated adolescents. Social Psychology Qwarterly, 52, 98-112.

Kaczmarek, M. G., \& Backlund, B. A. (1991). Disenfranchised grief: The loss of an adolescent romantic relationship. Adolescence, 26 (102), 253-259.

Kandel, D. B. (1978). Homophily, selection and socialization in adolescent friendships. American Joumal of Sociology, 84, 427-436.

Kandel, D. B. (1980). Drug and drinking behavior among youth. Annual Review of Sociology, 6 , 235-285.

Kandel, D. B., \& Andrews, K. (1987). Processes of adolescent socialization by parents and by peers. Intemational Joumal of the Addictions, 22, 319-342.

Kaplan, H. B., Martin, S. S., \& Robbins, C. (1984). Pachways to adolescent drug use: Selfderogation, peer influence, weakening of social controls, and early substance use. Jowmal of Healub and Social Behavior, 25, 270-289.

Keefe, K. (1992). Perceptions of normative social pressure and attitudes toward alcohol use: Changes during adolescence. Journal of Studies on Alcobol, 55, 46-54.

Kelly, J. A., \& Hansen, D. J. (1987). Social interactions and adjustment. In V. B. Van Hasselt \& M. Hersen (Eds.), Handbook of adolescent psychology (pp. 131-146). New York: Pergamon Press.

Kelman, H. C. (1974). Attitudes are alive and well and gainfully employed in the sphere of action. American Psycbologist, 29, 310-324.

King, J., Beals, J., Manson, S. M., \& Trimble, J. E. (1992). A structural equation model of factors related to substance use among American Indian adolescents. Drugs and Society, $6,253-268$.

Kirscht, J. P., Janz, N. K., Becker, M. H. (1989). Psychosocial predictors of change in cigarette smoking. Jourmal of Applied Social Psychology, 19, 298-308.

Klitzner, M., Greunewald, P. J., \& Bamberger, E. (1990). The assessment of parent-led prevention programs: A preliminary assessment. Joumal of Drug Education, 20, 77-94. 
Knibbe, R. A., Drop, M. J., \& Muytjens, R. A. (1987). Correlates of changes in the progression from everyday drinking to problem drinking. Sacid Science and Medicine, 24, 463-473.

Knibbe R. A., Oostveen, T., \& Van de Goor, I. (1991). Young peoples alcohol consumption in public drinking places: Reasoned behaviour or related to the situation. British Joumal of the Addiction, 86, 1425-1433.

Knibbe, R. A., \& Van de Goor, I. (1988). Modellen voor alcoholpreventie. In J. C. Van der Stel \& W. R. Buisman (Eds.), Alcoholpreventie: Achtergronden, praktijk en prewentie (pp. 85. 108). Alphen aan den Rijn: Samsom.

Knibbe, R. A., Van de Goor, I., \& Drop, M. J. (1993). Contextual influences on young people"s drinking rates in public drinking places: An observational study. Addiction Research, 1 , 269-278.

Kozlowski, L. T., \& Heatherton, T. F. (1990). Self-report issues in cigarette smoling: State of the art and future directions. Behavioral Assessment, 12, 53-75.

Krall, E. A., Valladian, I., Dwyer, J. T., \& Gardner, J. (1989). Accuracy of recalled smoking data. American Journal of Public Health, 79, 200-202.

Krosnick, J. A., \& Judd, C. M. (1982). Transitions in social influence at adolescence: Who induces cigarette smoking. Developmental Psychology, 18, 359-368.

Kuipers, S. B. M., Mensink, C., \& De Zwart, W. M. (1993). Jeugd en riskant gedrag (Youth and risk behawior). Utrecht: NIAD.

Kumpfer, K. L., \& Turner, C. W. (1990). The social ecology madel of adolescent substance abuse: Implications for prevention. International Joumal of the Addictions, 25, 435-463.

Lau, R. R., Quadrel, M. K., \& Hartman, K. A. (1990). Development and change of young adults' preventive health beliefs and behavior: Influence from parents and peers. Joumal of Health and Social Bebavior, 31, 240-259.

Lawrance, L. (1989). Validation of a self-efficacy scale to predict adolescent smoking. Health Education Research, 4, 351-360.

Lawrance, L., \& Rubinsom, L. (1986). Self-efficacy as a predictor of smoking behavior in young adolescents. Addictive Behaviors, 11, 367-382.

Leegwater, M. A. B. (1996). Drink-en rijgedrag wan discotbeekbezoekers in de provincie Groningen (Drinking and driving of visitors of discos in Groningen). Thesis. Maastricht University.

Leifman, H., Kühlhorn, E., Allebeck, P., Andréasson, S., \& Romesjö, A. (1995). Abstinence in late adolescence - antecedents to and covariates of a sober lifestyle and its consequences. Social Science and Medicine, 41, 11-121.

Leigh, B. C., \& Aas, H. (1995). Twoyear longitudinal stady of alcobol expectancies and drinking: among Norwegian adolescents. Paper presented at the $21^{\text {th }}$ Annual Alcohol Epidemiology Symposium of the Ketril Bruun Society. O'Porto, Portugal.

Leigh, B. C. Schafer, J., \& Temple, M. T. (1995). Alcohol use and contraception in first sexual experiences, Joumal of Bebavioral Medicine, 18, 81-95.

Lemmens, P. H. H. M., \& Drop, R. A. (1995/1996). Op zoek naar de gezonde drinker. Tidschrift voor Alcobol, Drugs en andere Pychotrope Stoffen, 21, 226- 234.

Leventhal, H., \& Cleary, P. D. (1980). The smoking problem: A review of the research and theory in behavioral risk modification. Psychological Bulletin, 88, 370-405.

Leventhal, H., Keeshan, P., Baker, T., \& Wetter, D. (1991). Smoking prevention: Toward a process approach. British Joumal of Addiction, 86,583-587.

Lichtenstein, E., \& Cohen, S. (1990). Prospective analysis of two modes of unaided smoking cessation. Healtb Education Research, 5, 63-72.

Lopes, L. L. (1993). Reasons and resources: the human side of risk taking. In N. J. Bell \& R. W. Bell (Eds.), Adolescent risk taking (pp. 29-54). Newbury Park, CA: Sage Publications. 
Maddox, G. (1962). Teenage drinking in the United States. In D. Pittman a C. Snyder (Eds.), Society, culture and drinking patterns. New York: Wiley and Sons.

Maisto, S. A., McKay, J. R., \& Connors, G. J. (1990). Self-report issues in substance abuse: State of the ant and future directions. Behavioral Assessment, 12, 117-134.

Mäkelä, K. (1997). Drinking, the majority fallacy, cognitive dissonance and social pressure. Addiction, 92, 729-736.

Makkelï, K., \& Mustonen, H. (1996). The reward structure of drinking among young and older male drinkers. Contemporary Drug Problems, 23,479-492.

Mann McLaughlin, L., Chassim, L., Sher, K. J. (1987). Alcohol expectancies and the risk for alcoholism. Joumal of Consulting and Clinical Psycbology, 55, 411-417.

Marks, $G_{\text {., }} \&$ Miller, N. (1987). Ten years of research on the false-consensus effect: An empirical and theoretical review. Pycbological Bulletin, 102, 72-90.

Marlatt, G. A., Curry, S., \& Gordon, J. R. (1988). A longitudinal analysis of unaided smoking cessation. Joumal of Consulting and Clinical Psychology, 56, 715-720.

Martin, G. L., \& Newman, I. M. (1988). Assessing the validity of self-reported adolescent cigarette smoking. Joumal of Drug Education, 18, 275-284.

Massey, J. L., \& Krohn, M. D. (1986). A longitudinal examination of an integrated social process model of deviant behavior. Social Forces, 65, 106-133.

Mayhew, D. R., Donelson, A. C., Beirness, D. J., \& Simpson, H. M. (1986). Youth, alcohol and relative risk of crash involvement. Accident Analysis and Prevention, 18, 273-287.

McGee, R., \& Stanton, W. R. (1993). A longitudinal study of reasons for smoking in adolescence. Addiction, 88, 265-271.

McLean, A. L. \& Fllanigan, B. J. (1993). Transition-marking behaviors of adolescent males at first intercourse. Adolescence, 28 (111), 579-595.

McNeill, A. D. (1991). The development of dependence on smoking in children. British Joumal of Addiction, 86, 589-592.

McNeill, A. D., Jarvis, M. J., Stapleton, J. A., Russell, M. A. H., Eiser J. R., Gammage, P., \& Gray, E. M. (1988). Prospective study of factors predicting uptake of smoking in adolescents. Joumal of Epidemiology and Community Health, 43, 72-78.

Midanik, L. (1982). The validity of self-reported alcohol consumption and alcohol problems: $A$ literature revilew. British Journal of Addiction, 77, 357-382.

Midanik, L. T. (1988). Validity of self-reported alcohol use: A literature review and assessment. British Joumal of Addiction, 83, 1019-1029.

Milgram, G. G. (1993). Adolescents, alcohol and aggression. Journal of Situdies on Alcohol, 11, 53-61.

Miller, L. C., Bettencourt, B. A., DeBro, S. C., \& Hoffman, V. (1993). Negotiating safer sex: Interpersonal dymamics, In J. Pryor \& G. Reeder (Eds.), The sacial psychology of AIDS infection (pp. 85-123). Hillsdale, NJ: Erlbaum.

Mills, C. J., \& Noyes, H. L. (1984). Patterns and correlates of initial and subsequent drug use among adolescents. Joumal of Consulting and Clinical Pycbology, 52, 231-243.

Molimard, R. (1995). Unknowns. In K. Slama (Ed.), Tobacco and Health (pp. 449-453). New York: Plenum Press.

Moore, D. (1987). Parent-adolescent separation: The construction of adulthood by late adolescents. Developmental Pychology $23,298-307$.

Moos, R. H., Finney, J. W., \& Gamble, W. (1982). The process of recovery of alcoholism. II. Comparing spouses of alcoholic patients and matched community controls. Journal of Studies on Alcohol, 43, 888-909. 
Morgan, M., \& Grube, J. W. (1991). Closeness and peer group influence. British Joumal of Social Pycbology, 30, $159-169$.

Mosbach, P., \& Leventhal, H. (1988). Peer group identification and smoking: Implications for intervention. Jowrmal of Abromal Psychology, 97, 238-245.

Mudde, A. N. (1994). The dewelopment and eualuation of a communty and a mass media approach to smoking cessation. Dissertation. Maastricht: University Press.

Murray, M., Swan, A. V., Johnson, M. R., \& Bewley, B. R. (1983). Some factors associated with increased risk of smoking by children. Jommal of Child Psychology and Psychiatry and Alied Disciplines, 24, 223-232.

Murray, M., Swan, A. V., Kiryluk, S., \& Clarke, G. C. (1988). The Hawthorne effect in the measurement of adolescent smoking. Joumal of Epidemiology and Community Health, $42,304-306$.

Myers, M. G., \& Brown, S. A. (1994). Smoking and heatth in substance-abusing adolescents: $A$ two-year follow-up. Pediatrics, 93, 561-566.

National Health and Medical Research Council (1987). Is there a safe level of dally consumption of alcobal for men and women? Recommendations regarding responsible drinking behatitour. Canberra: Australian Government Publishing Service.

Needle, R, McCubbin, H., Lorence, J., \& Hochhauser, M. (1983). Reliability and validity of adolescent self-reported drug use in a family based study: A methodological study. International Jowmal of the Addictions, 18, 901-912.

Needle, R., McCubbin, H., Wilson, M., Reineck, R., Lazar, A., \& Mederer, H. (1986). Interpersonal influences in adolescent substance use: 'The role of older siblings, parents and peers. International joumal of the Addictions, 21, 739-766.

Newcomb, M. D., \& Bentler, P. M. (1986). Frequency and sequence of drug use: A longitudinal study from early adolescence to young adulthood. Journal of Drug Education, 16, 101120.

Newcomb, M. D., \& Bentler, P. M. (1988). Consequences of adolescent drug use: Impact on the lives of young people. Newbury Park: Sage.

Newcomb, M. D., McCarthy, W. J., \& Bentler, P. M. (1989). Cigarette smoking, academic lifestyle, and social impact efficacy: An eight-year study from early adolescence to young adulthood. Joumal of Applied Social Psychology, 19, 251-281.

Nolte, A. E., Smith, B. J., \& O'Rourke, T. (1983). The relative importance of parental attitudes and behavior upon youth smoking behavior. Joumal of School Health, 53, 264-271.

O'Callaghan, F. V., \& Callan, V. J. (1992). Young adult drinking behaviour: A comparison of diary and quantity-frequency measures. British jourmal of Addiction, 87, 723-732.

Oei, T. P.S. \& Fae, A. (1987). Smoking prevention program for children: A review. Jourmal of Drug Education, $17,11-42$.

Oetting, E. R, Be Beauwais, F. (1986). Peer cluster theory: Drugs and the adolescent. Jourmal of Counseling and Development, 65, 17-22.

Oetting, E. R., \& Beauvais, F. (1987). Peer cluster theory, socialization characteristics, and adolescent drug use: A path analysis. Joumal of Counselling Psychology, 2, 205-213.

Office for National Statisties. (1997). Living in Britain: Prelliminary results from the 1996 General Household Survey. London: The Stationery Office.

O'Malley, P. M., Bachman, J. G., \& Johnston, L. D. (1983). Reliability and consistency in selfreports of drug use. International Jowmal of the Addictions, 18, 805-824.

Oostveen, T., Knibbe, R., \& De Vries, H. (1996). Social influences on young adults alcohol consumption: Norms, modeling, pressure, socializing and conformity. Addictive Bebaviors, 21, 187-197. 
Pallonen, U. E., Murray, D. M., Schmid, L., Pirie, P.y Luepker, R. V. (1990). Patterns of selfinitiated smoking cessation among young adults. Healt b Pychology, $9,418-426$.

Pape, H. (1997). Drinking, getting stoned ot staying sober: A general population study of alcohol consumption, cannabis we, drinking-related problems and sobriety among young men and women. Dissertation. Oslo: NOVA.

Pape, H., \& Hammer, T. (1996a). Sober adolescence: Predictor of psychosocial maladjustment in young adulthood? Scandinawian foumal of Psychology, 37, 362-377.

Pape, $H$, \& Hammer, T. (1996b). How does young people's alcohol consumption change during the transition to early adulthood? A longitudinal study of changes at aggregate and individual level. Addiction, 91, 1345-1358.

Passies, G. (1991). Alcoholgebruik bij jeugdige ongevalsslachtoffers (Alcohol use among young casualties of accidents). Tijidschrift voor Alcohol, Drugs en andere Psychotrope Stoffer, 17, 5-59.

Paul, E. L., \& White, K. M. (1990). The development of intimate relationships in late adolescence. Adolescence, 25 (98), 375-200.

Pedersen, W. (1993). The majority fallacy reconsidered. Acta Sociologica, 36, 343.

Pederson, L. L., \& Lefcoe, N. M. (1987). Short- and Jong-term prediction of self reported cigarette smoking in a cohort of late adolescents: Report of an 8-year follow-up of public school students. Prewentive Medicine, 16, 432-447.

Perry, C. L., Williams, C. L., Forster, J. L., Wolfson, M., Wagenaar, A. C., Finnegan, J. R., McGovern, P. G., Veblen-Mortenson, S., Komro, K. A., \& Anstine, P. S. (1993). Background, conceptualization and design of a community-wide research program on adollescent alcohol use: Project Northland. Health Education Research, 8, 125-136.

Petraitis, J., Flay, B. R., \& Miller, "T'. Q. (1995). Reviewing theories of adolescent substance use: Organizing pieces in the puzzle. Psychological Bulletin, 117,67-86.

Pierce, G. R., Sarason, B. R., \& Sarason, I. G. (1992). General and specific support expectations and stress as predictors of perceived supportiveness: An experimental study. Journal of Personality and Social Psychology, 63, 297-307.

Pierce, J. P., \& Gilpin, E. (1996). How long will today's new adolescent smoker be addicted to cigarettes. American Joumal of Public Health, 86, 253-256.

Pittman, D. I. (1995). What do we know abowt beneficial consequences of moderate alcobol consumpition on social and physical well-being? A critical review of the recent literature. Paper presented at the $1^{\text {th }}$ conference on Social and Health Effects of Different Drinking Patterns, Toronto, Canada, 1995.

Plant, M. A., \& Foster, J. (1991). Teenagers and alcohol: Results of a Scottish national survey. Drug and Alcobol Dependence, 28, 203-210.

Plant, M. A., Peck, D. F., \& Samuel, E. (1985). Alcohol drugs an school leavers. London: Tavistock Publications.

Power, C., \& Estaugh, V. (1990). The role of family formation and dissolution in shaping drinking behawior in early adulthood. British foumal of Addiction, $85,521-530$.

Presti, D. E., Ary, D. V., \& Lichtenstein, E. (1992). The context of smoking initiation and maintenance: Findings from interwiews with youths. Journal of Substance Abuse, 4, 3545.

Prochaska, J. O., \& DiClemente, C. C. (1983). Stages and processes of self-change of smoking: Toward an integrative model of change. Joumal of Consulting and Clinical Psychology, $51,390-395$.

Prochaska, J. O., DiClemente, C. C., \& Norcross, J. C. (1992). In search of how people change: Applications to addictive behaviors. American Py,chologist, 47, 1102-1114. 
Prokhorov, A. V., Pallonen, U. E., Fava, J. L., Ding, L., $8 x$ Niaura, R. (1996). Measuring nicotine dependence among high-risk adolescent smokers. Addictive Behavion, 21, 117 127.

Pulkkinen, L., \& Pitkanen, T. (1994). A prospective study of the precursors to problem drinking in young adulthood. Joumal of Studies on Alcobol, 55, 578-587.

Randall, D. M., \& Wolff, J. A. (1994). The time interval in the intention-behaviour relationship: Meta-analysis. British Joumal of Social Prycbology, 33, 405-418.

Reid, J. B. (1978). Study of drinking in natural settings. In G. A. Marlatt \& P. E. Nathan (Eds.), Behavioral approaches to alcabolism. New Brunswick, NJ: Rutgers Center of Alcohol Studies.

Rose, J. S., Chassin, L., Presson, C. C., \& Sherman, S. J. (1996). Prospective predictors of quit attempts and smoking cessation in young adults. Health Psychology, 15, 261-268.

Rundall, T. G., \& Bruvold, W. H. (1988). A meta-analysis of school based smoking and alcohol use prevention programs. Health Education Quarterly, 15, 317-334.

Rusbult, C. E. (1983). A longitudinal test of the investment model: The dewelopment (and deterioration) of satisfaction and commitment in heterosexual involvements. Joumal of Personality and Social Psychology, 45, 101-117.

Rusbult, C. E., Verette, J., Whitney, G. A., Slovik, L. F., \& Lipkus, I. (1991). Accommodation processes in close relationships: Theory and preliminary empirical evidence. Joumal of Personality and Social Psychology, 60, 53-78.

Samet, N., \& Kelly, E. (1987). The relationship of steady dating to self-esteem and sex role identity among adolescents. Adolescence, 22(85), 231-245.

Sanderson, C. A., \& Cantor, N. (1995). Sociall dating goals in late adolescence: Implications for safer sexual activity. Journal of Personality and Sacial Psychology, 68, 1121-1134.

Schaalma, H. P. (1995). Planned development and evaluation of scbool-based AIDS/STD education. Dissertation. Maastricht.

Schulenberg, J., Wadsworth, K. N., OMalley, P. M., Bachman, J. G., \& Johnston, L. D. (1996). Adolescent risk factors for binge drinking during the transition to young adulthood: Variable- and pattern-centered approaches to change. Developmental Psychology, 32, 659-674.

Selnow, G. W., \& Crano, W. D. (1986). Formal vs informal group affiliations: Implications for alcohol and drug use among adolescents. Journal of Studies on Alcobol, 47, 48-52.

Semmer, N. K., Dwyer, J. H., Lippert, P., Fuchs, R., Cleary, P. D., \& Schindler, A. (1987). Adolescent smoking from a functional perspective: The Berlin-Bremen Study. Europear Jowmal of Psychology of Education, 2,387-401.

Sherman, S. J., Presson, C. C., Chassin, I., Corty, E., \& Olshavsky, R. (1983). The false consensus effect in estimates of smoking prevalence: Underlying mechanisms. Pers. ortality and Social Psychology Bulletin, 9, 197-208.

Shiffman, S. (1989). Trans-situational consistency in smoking relapse. Health Psychology, 8, 471481.

Shiffman, S., \& Jarvis, M.E. (1987). Situationall determinants of coping in smoking relapse crises. Journal of Applied Social Psycbology, 17, 3-15.

Silbereisen, R. K., \& Noack, P. (1988). On the constructive role of problem behavior in adollescence. In N. Bolger (Ed.), Persons in context. Developmental processes (pp. 153-180). Cambridge: Cambridge University Press.

Silbereisen, R. K., Noack, P., \& Von Eye, A. (1992). Adolescents' development of romantic friendship and change in fawourite leisure contexts. Joumal of Adolescent Research, 7, 80. 93. 
Simons, R. $_{\text {. }}$, Conger, R. D., \& Whitbeck, L. B. (1988). A multistage social learning model of the influences of family and peers upon adolescent substance abuse. Jowmal of Dhug Issues, $18,293-315$.

Single, E., Kandel, D. B., \& Johnson, B. D. (1975). The reliability and validity of drug use responses in a large scale longitudinal study. Joumal of Drug Issues, $5,426-443$.

Skinner, W. F., Massey, J. L., Krohn, M. D., \& Lauer, R. M. (1985). Social influences and constraints on the initiation and cessation of adolescent tobacco use. fourrat of Behavioral Medicine, 8, 353-376.

Skog, O.J. \& Duckert, F. (1993). The development of alcoholics' and heavy drinkers" consumption: A longitudinal study. Jonmal of Studies on Alcobol, 5A, 178-188.

Sobell, L. C., \& Sobell, M. A. (1992). Timeline follow-back: A technique for assessing selfreported alcohol consumption. In R. Z. Lirten $8 x \mathrm{~J}$. P. Allen. (Eds.), Measuring alcobol consumption: Psychosocial and biochemical methods (pp. 41-72). Totowa, NJ: Humana Press, Inc.

Sobell, L. C., Sobell, M. B., Leo, G. L, \& Cancilla, A. (198.8). Reliability of a timeline method: Assessing normal drinkers reports of recent drinking and a comparative evaluation across several populations. British Journal of Addiction, 83, 393-402.

Social en Culturee] Planbureau. (1994). Rappontage jeugd 1994 [Reports on youth in the Netherlands. Den Haag: VUGA.

Stacy, A. W., Bentler, P. M., \& Flay, B. R. (1994). Attitudes and health behavior in diverse populations: Drunk driving, alcohol use, binge eating, marijuana use, and cigarette use. Health Psychology, 13,73-85.

Stacy, A. W., Widaman, K. E., \& Marlatt, G. A. (1990). Expectancy models of alcohol use. Jownal of Personality and Social Psychology, 58, 918-928.

Stanton, W. R. (1995). DSM-III-R tobacco dependence and quitting during late adolescence. Addictive Bebaviors, 20, 595-603.

Stanton, W. R., Currie, G. D., Oei, T. P. S., \& Silva, P. A. (1996). A developmental approach to influences on adolescents' smoking and quitting. Jaumal of Applied Developmental Psychology, 17, 307319 .

Stein, J. A., Newcomb, M. D., \& Bentler, P. M. (1987). An 8-year study of multiple influences on drug use and drug use consequences. Joumal of Personality and Social Psychology, 53, 1094-110.5.

Stein, J. A., Newcomb, M. D., \& Bentler, P. M. (1996). Initiation and maintenance of tobacco smoking: Changing personality correlates in adolescence and young adulthood. Journal of Applied Social Psychology, 26, 160-187.

Stern, R. A., Prochaska, J. O., Velicer, W. F., \& Elder, J. P. (1987). Stages of adolescent cigarette smoking acquisition: Measurement and sample profiles. Addictive Behaviors, $12,319-329$.

Stone, S. Im, \& Kristeller, J. L. (1992). Attitudes of adolescents toward smoking cessation. American Joumal of Preventive Medicine, 8, 221-225.

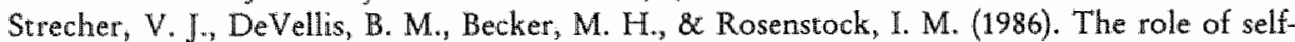
efficacy in achieving health behavior change. Health Education Research, 13, 73-91.

Sutton, S. R. (1987). Social-psychological approaches to understanding addictive behaviours: Attitude-behaviours and decision-making modells. British Joumal of Addiction, 82, 355370.

Sutton, S. R. (1992). Is taking up smoking a reasoned action. Addiction, 87, 21-24.

Sutton, S. R. (1995). Translating a desire to stop smoking into decision and action. In K. Slama (Ed.), Tobacco and bealth. New York: Plenum. 
Telch, M. J., Miller, L. M., Killen, J. D., Cooke, S., \& Maccoby, N. (1990). Social intluence approach to smoking prevention: The effects of videotape delivery with and without same-age peer leader participation. Addictive Bebaviors, 15, $21-28$.

Temple, M. T., \& Fillmore, K. M. (1985-86). The variability of drinking patterns and problems among young men, age 16-31: A longitudinal study. International foumat of the Addictions, 20, 1595-1620.

Temple, M. T., Fillmore, K. M., Hartka, E., Johnstone, B., Leino, E. V., \&. Motoyoshi, M. (1991). A meta-analysis of change in marital and employment status as predictors of alcohol consumption on a typical occasion. British yowmal of Addiction, 86, 1269-1281.

Thombs, D. L., \& Beck, K. H. (1994). The social context of four adolescent drinking patterns. Health Education Research, 9, 13-22.

Todd, G. F., \& Laws, J. T. (1959). The reliability of statements about smoking babits. London: Tobacco Manufacturers Standing Committee.

Trzen, B., \& Nordlund, S. (1993). Visiting public drinking places in Oslo: An application of the theory of planned behaviour. Addiction, 88, 1215-1224.

Triandis, H. C. (1980). Values, attitudes and interpersonal behavior. In H. E. Howe, Jr. \& M. M. Page (Eds.), Nebraska Symposinm on Motivation 1979 (vol. 27, pp. 195-259). Lincoln: University of Nebraska Press.

Tuakli, N., Smith, M. A., \& Heaton, C. (1990). Smoking in adolescence: Methods for health education and smoking cessation. fournal of Family Practice, 31, 369-374.

Turner, J. C. (1991). Social influence. Pacific Grove CA: Brooks/Cole Publishing Co.

Urberg, K. A., Cheng, C. H., \& Shyu, S. J. (1991). Grade changes in peer influence on adolescent cigarette smoking: A comparison of two measures. Addictive Behations, 16, 21-28.

Urberg, K. A., Degirmenciogly, S. M., \& Pilgram, C. (1997). Close friend and group influence on adolescent cigarette smoking and alcohol use. Dezelopmental Psychology, 33, 834-844.

U.S. Department of Health and Human Services (1997). Preliminary results from the 1996 national household survey on drug abuse. Washington DC: U.S. Government printing office.

U.S. Surgeon General (1991). Surgeons general's report on the health benefits of smoking cessation. Public Health Reports, 105, 545-548.

Van de Goor, L. A. M. (1990). Situational aspects of adolescent drinking behavior. Dissertation. Meppel: Krips Repro.

Van Reek, J., Knibbe, R. A., \& Van Iwaarden, T. (1993). Policy elements as predictors of smoking and drinking behaviour: The Dutch Cohort Study of secondary schoolchildren. Health Policy, 26,5-18.

Wagner, E. F. (1993). Delay of gratification, coping with stress, and substance use in adolescence. Special section: Motivation and addictive behaviors. Experimental and Clinical Psycbopbarmacology, 1, 27-43.

Warheit, G. J., Biafora, F. A., Zimmerman, R. S., Gil, A. G., Vega, W. A., \& Apospori, E. (1995). Self-rejection/derogation, peer factors, and alcohol, drug, and cigarette use among a sample of Hispanic African-American, and White non-Hispanic adolescents. International Journal of the Addictions, 30, 97-116.

Webster, R. A., Hunter, M., \& Keats, J. A. (1994). Personaliry and sociodemographic influences on adolescents" substance use: A path analysis. International Journal of the Addictions, 29, 9411-956.

Weinstein, $E_{.}$\& Rosen $E_{.}$(1991). The development of adolescent sexual intimacy: Implications for counselling. Adolescence, 26(102), 331-339. 
Weiss, S., \& Moore, M. (1994). Why, where and with whom do Israeli teenagers drink? 'To whom do they turn for help with alcohol problems? Alcobol and Alcobolism, 29, $465-471$.

Werch, C. E., \& Anzalone, D. (1995). Stage theory and research on tobacco, alcohol, and drug use. Joumal of Drug Education, 25, 81-98.

Werner, M. J. (1991). Adolescent substance abuse: Risk factors and prevention strategies. Maternal and Cbild Health Technical Information Bulletin, 1-16.

Wiers, R. W. (1998). Bad expectations? Cognitive and nexropsychological indicators of enhanced risk for alcobolism. Dissertation. Delft: Eburon.

Wilks, I.y \& Callan, V. J. (1987). Expectations about appropriate drinking contexts: Comparison of parents, adolescents and best friends. British Joumal of Addiction, 83 , 1055-1062.

Wilks, J., Callan, V. J., \& Austin, D. A. (1989). Parent, peer and personal determinants of adolescent drinking. British Joumal of Addiction, 84, 619-630.

Willemsen, M. C. (1997). Kicking the babit: The effectiveness of smoking cessation programs in Dutch work sites. Dissertation. Maastricht: Unigraphic.

Williams, J. G., \& Smith, J. P. (1994). Drinking patterns and dating violence among college students. Prychology of Addictive Bebaviors, 8, 51-53.

Wills, T. A. (1994). Self-esteem and perceived control in adolescent substance use: Comparative tests in concurrent and prospectiwe analyses. Psychology of Addictive Behaviors, 8 , 223-234.

Wills, T. A., \& Cleary, S. D. (1996). How are social support effects mediated? A test with parental support and adolescent substance use. Joumal of Personality and Social Psychology, 71, 937-952.

Windle, M., \& Windle, R. C. (1996). Coping strategies, drinking motiwes, and stressful life events among middle adolescents: Associations with emotional and behavioral problems and with academic functioning. Joumal of $A$ bnomal Psychology, 105, 551-560.

Winefield, H. R., Winefield, A. H., Tiggeman, M., \& Goldney, R. D. (1989). Psychological concomitants of tobacco and alcohol use in young Australian adults. British Jowmal of Addiction, 84, 1067-1073.

Wong, M. M., \& Csikszentmihalyi, M. (1991). Affiliation motivation and daily experience: Some issues on gender differences. Joumal of Persomality and Social Psychology, 60, $154-164$.

World Health Organization (1983). Guidelines for the canduct of tobacco smoking surveys of the general population. Geneva.

Yamaguchi, K. (1990). Drug use and its social covariates from the period of adolescence to young adulthood: Some implications from longitudinal studies. In M. Galanter (Ed.), Recent developwents in alcoholism. Vol. 8. Combined alcohol and otber drug dependence (pp. 205-219). New York: Plenum Press.

Yamaguchi, K., \& Kandel, D. B. (1985). On the resolution of role incompatibility: A life event history analysis of family roles and marijuana use. American Journal of Saciology, 90 , $1284-1325$. 


\section{SAMENVATTING}

Adolescentie is de periode waarin veel jongeren experimenteren met potentieel riskante gewoonten zoals roken, drinken en druggebruik. Een belangrijke vraag is of jongeren die beginnen te roken of drinken zijn te onderscheiden van degenen die dit niet gaan doen. En welke factoren bepalen of jongeren die experimenteren stoppen, doorgaan met gebruik of zelfs overmatig gaan gebruiken. Om dit soort vragen te onderzoeken is een longitudinaal design vereist waarin een groep voordat ze beginnen met gebruik worden geïnterviewd en vervolgens een aantal malen gedurende de adolescentie. Dit proefschrift beschrijft de resultaten van zo'n langlopend onderzoek onder jongeren. Drie aspecten van roken en drinken staan centraal in deze studie. Allereerst is onderzocht wat de rol is van de directe omgeving van jongeren (ouders, broers en zussen, vrienden, partner) bij de ontwikkeling van roken en drinken. Verder is onderzocht wat mogelijke positieve functies van alcoholgebruik voor adolescenten zijn. Verwacht wordt dat inzicht in de voordelen van gebruik licht zal doen schijnen op de redenen van jongeren om te gaan drinken of dit te blijven doen. Ten derde gaat dit proefschrift in op het relatieve effect van verwachtingen van jongeren over roken op het beginnen met roken en op het stoppen met roken. Het longitudinale karakter van onze studie gaf mogelijkheden om ook meer methodologisch georiënteerde vraagstellingen te stellen. Bijvoorbeeld, kunnen factoren die op één moment samenhangen met roken tevens het beginnen met roken enige jaren later verklaren? Een andere vraag is of rapportages over het eigen rook-en drinkgedrag wel betrouwbaar zijn. In het eerste hoofdstuk wordt ingegaan op de achtergronden van de diverse aspecten van onze 
studie. De nadruk ligt op theoretische inzichten en eerder verricht onderzoek. Daarnaast wordt duidelijk gemaakt welke problemen er nog zijn en hoe die kunnen worden aangepakt. Hierdoor wordt duidelijk in hoeverre het onderhavige project aansluit bij en voorborduurt op eerder onderzoek.

In het najaar van 1989 is de cohort-studie gestart met het afnemen van een vragenlijst bij 1454 eerste en tweedejaars scholieren van 36 middelbare scholen. De keuze van de scholen was zo gemaakt dat er een spreiding was naar regio in Nederland en naar opleidingsniveau (variërend van LBO tot VWO). Twee jaar later in 1991 werden de jongeren via de ouders thuis benaderd met het verzoek een tweede vragenlijst in te vullen. In 1994, vijf jaar na de eerste meting, werden de respondenten opnieuw benaderd. In totaal namen 1063 personen deel aan het volledige onderzoek. In hoofstuk 2 wordt uitvoerig de opzet en uitvoering van de studie besproken. Daarnaast wordt in dat hoofdstuk de ontwikkeling van rook- en drinkgedrag geschetst. Uit het onderzoek bleek dat, terwijl op een leefrijd van 12, 13 jaar nog maar een paar procent van de jongeren dagelijks rookt, wijf jaar later ongeveer een derde dagelijks rookt. Het percentage jongeren dat regelmatig alcohol gebruikt stijgt nog sterker: dit loopt op van 3\% tot meer dan 50\%. Deze gegevens geven echter niet aan of iemand die, bijvoorbeeld relatief veel drinkt op jonge leeftijd, een aantal jaren later ook een hoog alcoholgebruik heeft. In de literatuur is er geen overeenstemming over het antwoord op de vraag of roken en drinken in vroege adolescentie leidt tot verhoogd gebruik en misbruik in late adolescentie. Volgens ons wordt deze onduidelijkheid veroorzaakt door verschillen in methoden die onderzoekers gebruiken om dit te onderzoeken. Uit onze gegevens blijkt dat de relatieve frequentie van roken en drinken gelijk blijkt. Echter, als specifiek wordt gekeken naar het drinkniveau of aantal sigaretten dat wordt gebruikt, blijkt dit van weinig voorspellende waarde te zijn voor het niveau van gebruik enige jaren later.

In hoofdstuk 3 wordt nagegaan in hoeverre gelijkenissen van rookgedrag in vriendengroepen worden bepaald door beïnloedingsprocessen en door selectieprocessen. In veel studies worden sterke gelijkenissen in gedragingen binnen vriendenkringen toegeschreven aan de invloed van vrienden. Verondersteld wordt dat een jongere die rookt en veel vriendjes heeft die roken is aangezet om te gaan roken. Niettemin kunnen gelijkenissen in rookgedrag ook tot stand komen doordat jongeren specifieke vriendschappen aangaan met personen die dezelfde levensstijl hebben (selectie), of doordat verschillen in gedragingen ertoe leiden dat personen met afwijkend (rook-en drink) gedrag uit een groep komen te vallen (deselectie). In de meeste studies wordt geen rekening gehouden met de mogelijkheid van selectieprocessen. Uit ons onderzoek blijkt dat met name selectie een belangrijke verklaring biedt voor homogeniteit in rookgedrag in een vriendengroep. Voor de invloed van vrienden wordt slechts zeer beperkt steun gevonden en er wordt geen steun gevonden voor processen van afwijzing. De bevindingen die aansluiten bij recent Amerikaans onderzoek, tonen aan dat de aandacht voor de invloed van vrienden in theoretische modellen moet worden genuanceerd. Indien in onderzoek 
geen aandacht wordt besteed aan selectieprocessen zijn uitspraken over het effect van vrienden op gedrag van adolescenten prematuur.

Hoofdstuk 4 beschrijft een studie naar de relatieve invloed van ouders en vrienden op veranderingen in rook- en drinkgedrag van jongeren. Een analytisch model is ontwikkeld dat rekening houdt met selectieprocessen. Het gedrag van de twee beste vrienden blijkt marginaal (alcohol) of niet (roken) veranderingen in rooken drinkgedrag van respondenten tot gevolg te hebben. Ouders daarentegen hebben een effect op het gedrag van hun kinderen. Wel moet gezegd worden dat de invloed van ouders aanwezig niet sterk is. Verder lijkt het erop dat ouders het gedrag van vrienden van hun kinderen beïnloeden. Ouders lijken door hun meningen, eigen gedragingen en levensstijl niet alleen invloed te hebben op het gedrag van hun kinderen, maar tevens op hun keuze van vrienden.

Naast familie en vrienden is een derde partij mogelijk belangrijk in thet leven van adolescenten, namelijk een partner. In hoofdstuk 5 wordt nagegaan of de aanwezigheid van een vaste relatie het drinkpatroon van jongeren beïnloedt. Uit een vergelijking van het drinkniveau bij de tweede meting met dat bij de derde meting bleek dat vooral bij jongens, een vaste vriendin invloed heeft op ontwikkeling van alcoholgebruik. Jongens met een vaste vriendin gaan relatief minder drinken in openbare drinkgelegenheden zoals kroegen en discotheken, maar gaan meer thuis drinken in vergelijking met jongens zonder vaste vriendin. Bij meisjes lijkt een vaste vriend een minder prominente functie te hebben in relatie tot veranderingen in alcoholgebruik.

Het tweede deel van hoofstuk 5 en de hoofdstukken 6 en 7 gaan in op functies van drankgebruik bij adolescenten. Volgens sommige onderzoekers is drinken niet a priori abnormaal of irrationeel: alcoholgebruik kan bepaalde doelen vervullen en een bijdrage leveren an de psychosociale ontwikkeling van jongeren. Kortom, drinken kan gepaard gaan met andere verschuivingen in het leven van adolescenten die een positieve betekenis hebben. In de literatuur is een aantall functies te onderscheiden waarvan verondersteld wordt dat ze samenhangen met drinken namelijk (a) bekend raken met waarden en gedragingen van volwassenen op gebied van zelfstandig functioneren maar ook op het gebied van werk en opleiding, (b) adequaat functioneren in een vriendengroep, (c) ontwikkelen van intieme relaties en (d) psychologisch welbevinden. De bevindingen uit hoofdstuk 5 geven aan dat jongeren die drinken in mid-adolescentie (14-15 jaar) meer kans hebben op het hebben van een vaste partner in late adolescentie (17-18 jaar). Met name het gebruik in gelegenheden waar de andere sekse aanwezig is zoals café's, disco's, op feestjes, leidt tot een verhoogde kans op ontwikkeling van intieme relaties. Het mag niet verwonderen dat drinken thuis, samen met de ouders, geen effect heeft op relatievorming.

Uit het voorgaande blijkt al dat de context van gebruik een relevante factor is in de studie van functies van alcoholgebruik. Hoofdstuk 6 richt zich op de situatie van gebruik en beschrijft een vergelijking tussen jongeren die uitgaan met jongeren 
die niet uitgaan. Bezoekers van uitgaansgelegenheden rapporteerden meer 'transitie gedragingen'; ze hadden bijwoorbeeld vaker werk en ook vaker een partner dan degenen die geen uitgaansgelegenheden bezoeken. Daarnaast is deze groep beter geintegreerd in de vriendenkring; ze hebben niet alleen meer vrienden maar ze geven aan beter contact te hebben met hen en zich minder vaak alleen te voelen. Geen verschillen werden gevonden voor stress en zelfwaardering.

Als inderdaad de context van gebruik belangrijk is bij het bepalen van functies van drinken woor jongeren, komt de vraag op waarom sommige jongeren dan alleen maar thuis drinken. De genoemde sociale voordelen op het terrein van vriendschap en romantische relaties gaan immers niet op voor deze groep. In hoofdstuk 7 wordt onderzocht of juist niet in deze groep, jongeren drinken om 'negatieve' redenen, namelijk om om te gaan met gevoelens van stress en ontevredenheid met zichzelf. Analyse van de relaties tussen drinkniveau enerzijds en stress en zelfvertrouwen anderzijds, geeft aan dat alleen in de groep van mannelijke 'thuisdrinkers' wordt gedronken om negatieve gevoelens te vergeten. Bij de andere groepen (degenen die uitsluitend buitenshuis drinken, of in beide situaties drinken) zijn er geen aanwijzingen voor drinken als manier om met problemen om te gaan. Bij meisjes werden geen negatieve effecten van thuisdrinken gevonden.

Hoofdstuk 8 gaat in op de voorspellers van het beginnen met roken door jongeren. Hiervoor werd gebruik gemaakt van drie categorieën van factoren namelijk rook-gerelateerde cognities zoals attitude en verwachtingen over de eigen effectiviteit, rookgedrag van anderen en sociaal demografische variabelen zoals geslacht en opleiding. Centraal staat de vergelijking tussen cross-sectionele en longitudinale analyses. Deze vergelijking geeft ons de mogelijkheid om na te gaan of factoren die op één moment samenhangen met roken ook veranderingen in rookstatus op termijn kunnen voorspellen. Rook-gerelateerde cognities (attitudes en eigen effectiviteit) en het roken van familieleden zijn factoren die niet alleen samenhangen met roken maar ook van voorspellende waarde zijn voor het beginnen met roken. Dus jongeren die van mening zijn dat roken meer voor- dan nadelen heeft, het moeilijk vinden om interne en externe invloeden om te roken te weerstaan en rokende familieleden hebben, zijn eerder geneigd te gaan roken. Echter, het gedrag van vrienden dat cross-sectioneel sterk samenhangt met roken van de respondent, heeft geen effect op het beginnen op de langere termijn (zie ook hoofdstukken 3 en 4). Opgemerkt moet worden dat de onderhavige factoren maar een beperkt deel van het beginnen van roken verkllaren. Op mogelijke verklaringen voor de relatief lage voorspelbaarheid wordt uitgebreid ingegaan.

Het stoppen met roken door jongeren wordt besproken in hoofdstuk 9 . Om na te gaan wat voorspellers zijn van stoppen met roken bij jongeren, is gebruikt gemaakt van een fase model. Zo"n fase model veronderstelt dat rokers geen homogene groep vormen maar te onderscheiden zijn naar personen in verschillende fasen van motivatie om te stoppen. Onderzocht werd in hoeverre attitudes en eigen effectiviteit, het rookgedrag en de waargenomen druk van familie en vrienden en 
habituele factoren (bijvoorbeeld de frequentie en intensiteit van gebruik) van regelmatige rokers in mid-adolescentie de motivatie tot stoppen in late adolescentie bepalen. Met name de habituele kenmerken en rook-gerelateerde cognities bepaalden of iemand absoluut niet was gemotiveerd om te stoppen, zich juist aan het voorbereiden was om te stoppen, of zelfs al gestopt was. De bevindingen suggereren dat interventieprogramma's voor jonge rokers kunnen worden gericht op het beïnvloeden van de ideeën van rokers over de voor-en nadelen van roken en stoppen en op de mogelijkheden die rokers zien om roken te weerstaan. Daarnaast is de aanpak van de verslaving zoals ook in veel stoppen-met-roken programma"s voor volwassenen wordt toegepast, ook voor adolescenten gewenst gezien de sterke impact van habituele factoren.

De meeste empirische studies baseren hun bevindingen op de rapportages van respondenten over het eigen gedrag en preferenties. Zonder observatie van het feitelijk gedrag is het moeilijk na te gaan in hoeverre informatie over eerder en actueel gebruik valide en betrouwbaar is. Hoofdstuk 10 beschrijft een onderzoek naar de betrouwbaarheid van de meting van de leeftijd waarop jongeren voor het eerst een sigaret roken of alcohol nutrigen. De gerapporteerde 'beginleeftijd' werd op drie tijdstippen gemeten. Over het algemeen bleek dat degenen die bij de eerste meting lieten weten te roken of te drinken en dus ook een bepaalde beginleeftijd aangaven, bij de tweede en derde metingen steeds hogere beginleeftijden rapporteerden. Slechts een beperkt aantal respondenten was consistent in de antwoorden of rapporteerde zelfs een lagere beginleeftijd. De onbetrouwbaarheid van deze maat heeft een duidelijke impact op zijn waarde als predictor-variabele van toekomstig gedrag. De waarschijnlijk meest betrouwbare schatring van de beginleeftijd, namelijk die gerapporteerd bij de eerste meting, was in veel gevallen niet gerelateerd aan rook- en drinkgedrag bij de derde meting.

In het afsluitend hoofdstuk wordt na een korte samenvatting van de voornaamste bevindingen van de studies in dir proefschrift ingegaan op de implicaties ervan voor verder onderzoek. Voor het onderzoek beschreven in de hoofdstukken 3 en 4 (relatieve invloed van vrienden op rook- en drinkgedrag), hoofstukken 5, 6 en 7 (functies van alcoholgebruik en uitgaan) en hoofdstuk 9 (stoppen met roken) zijn korte voorstellen voor verdere studie gedaan. Verwacht wordt dat hierin bepaalde inhoudelijke en methodologische tekortkomingen van de huidige studie, problemen die vaak ook aanwezig zijn in andere studies, kunnen worden aangepakt. Daarnaast wordt ingegaan op de mogelijke consequenties van de bevindingen voor preventie van roken en alcoholmisbruik. 
Figuur 1. Een samenvatting van de belangrijkste bevindingen van dit proefschrift

Hoofdstuk

- Of rook- en drinkgedrag van adolescenten voorspeld kan worden door het vroeger gedrag hangt af van de metingen van het gedrag en de analyse-strategieën die worden gebruikt.

- Gelijkenissen in rookgedrag binnen vriendenkringen zijn in sterke mate toe te schrijven aan selectieprocessen en in minder sterke mate aan beinvloedingsprocessen.

- Indien rekening wordt gehouden met selectieprocessen blijkt het rook- en drinkgedrag van ouders meer effect te hebben op veranderingen in het gedrag van jongeren dan gedrag van vrienden.

- De invloed wan ouders ligt gecompliceerder dan waak wordt aangenomen omdat ouders niet alleen direct hun kinderen beïnvloeden maar ook een rol spelen bij vriendschapskeuzes.

- Het krijgen van een vaste partner in de adolescentic heeft als gevolg dat jonge mannen relatief meer thuis gaan drinken en minder in publieke drinkgelegenheden.

- Uitgaan gaat samen met een goede integratie in de vriendenkring, een vaste partner en andere transities die de overgang van adolescentie naar jong volwassenheid kenmerken.

- Jongeren die uitgaan hebben niet minder stress of meer zelfvertrouwen dan jongeren die niet uitgaan.

- Bij jonge mannen die uitsluitend thuis drinken blijkt alcoholgebruik samen te hangen met stress en lage zelfwaardering. Bij mannen die ook of uitsluitend buitenshuis drinken wordt zo'n relatie niet gevonden.

- Factoren die sterk gerelateerd zijn aan rookgedrag (roken vrienden, attitude, eigen effectiviteit) zijn amper in staat het beginnen met roken na 3 of 5 jaar te voorspellen.

- De motivatie van jonge rokers om te stoppen met roken is gerelateerd aan de attitude, eigen effectiviteit en habituele factoren drie jaar eerder.

- Veel jonge rokers zijn absoluut niet gemotiveerd om te stoppen met roken.

- Het concept "leeftijd waarop voor het eerst gerookt of gedronken is" moet voorzichtig worden gebruikt omdat de betrouwbaarheid van meting onvoldoende is en de relaties met feitelijk gedrag inconsistent zijn. 


\section{ACKNOWLEDGEMENTS}

From 1989 on, 1454 adolescents participated in (parts of) our study on smoking and drinking. They spent many hours in the past years on the several questionnaires and interviews. I would like to thank them, and also their parents, teachers and 36 school boards for cooperation in this study.

Riet, in October 1994, we talked about my plans for the coming years. Thank you for giving me the feeling that it was too much to do in four years. I will remember your thorough way of reading and critical comments on the papers. Besides expertise and experience, the ability to motivate should be an essential characteristic of a supervisor. Flowers only bloom if you give them water. Ronald, you did this in an outstanding way. You motivated me to overload you with new ideas, versions of articles and research proposals throughout the years. To both of you, I am grateful for the freedom you gave me to choose my own subjects in this study. Hein, as a social psychologist, you supported me in my "battle" against two sociologists in the first years. I admire your openness for new views and insights.

Ypie, thanks for your precise work on collecting (and cleaning) the data of the third wave, and being my roommate in the first year. Jan and Frans, thanks for collecting the rich and overwhelming data sets of the first and second measurement. Gerard, you will of course realize that I almost went desperate because of the fact that '..it would be better if the analyses were conducted slightly different..' But finally, I enjoyed the moment that we discussed the methodological comments on our article by one of the referents. I am grateful to Lisbeth Hall, Bob Wilkinson and Rosemary Tunnicliffe for the necessary corrections of my English writing. 'Thanks to the members of the scientific committee: Gerjo Kok, Martijn Berger, Piet van den Brandt, Klaus Mäkelä and Theo Paulussen, for the time and energy they spent on reading this thesis.

Next, I would like to thank Maaike and Edward for being my paranymphs. Maaike, you were a great support during the years in Maastricht (lots of scientific chats and gossip). Edward, you know that it is not a big step from evoking spirits, dealing with Mrs J. in Slovakia to doing research on juvenile drinking. Klaasjan and Rudie, thanks for our discussions about alcohol research, the comments on my papers, being my roommate (KJ), spending KBS-meetings together (Rudie) and the opportunity to learn from your promotion ceremonies. Paul, thanks for the efforts to keep me in the alcohol-field, our conversations and, of course, the postbadminton hours. Hannerieke, we shared an office room for only a short period; good luck with your research in the coming years. Special thanks to the other colleagues at Medical Sociology -- believe me, it is not easy for me to leave Maastricht. 
Although I dislike dissertations therein even the pet is thanked four times, this is a moment to thank Riet, Paul, Bas and Martijn. In the last months of finishing this thesis, I think a lot about my own puberty and the turbulent years on high school; thanks for your unqualified support. Furthermore, I'm grateful to my friendships, established in Groningen and Maastricht.

Guus, you let me realize that our working areas are not so different after all. Creativity is one of the most important requisites for doing research. Sophie, my little ghost, once you will know that things are seldom what they seem. 


\section{CURRICULUM VITAE}

Rutger Engels was born on October 24, 1968 in Velp. After finishing high school in Emmen, he went to the College for Social Work in Groningen for one year in order to study Psychology. Although he initially choose for Clinical Psychology, various experiences in the first years of the study made him to decide for Social Psychology. He participated in a research project on satisfaction and commitment in intimate relationships and in a project on fear-appeals in AIDS-preventive communication for promiscuous heterosexuals. In these years, he was chairman of the organization of Psychology students in Groningen and an assistant by different courses. After graduation in August 1993, he had several jobs varying from handyman to assistant of rheumatic patients in a health resort in Slovakia.

In May 1994, he started to work at the Department of Medical Sociology in Maastricht on the Ph.D project 'Determinants of changes in smoking and drinking of adolescents' described in this thesis. He was involved in teaching, in particular, in training of patient-physician communication for Medical students and supervision of students writing their Master thesis. In 1997, he was a member of the executive board of the department.

He has recently become a post-doc researcher at the Department of Youth, Family, and Life Course at the University of Utrecht. He will concentrate his research on peer-parent relationships in adolescence. 